\title{
Capillary Supercritical Fluid Chromatography
}

$$
\text { by }
$$

Jack Lee.Hensley, II

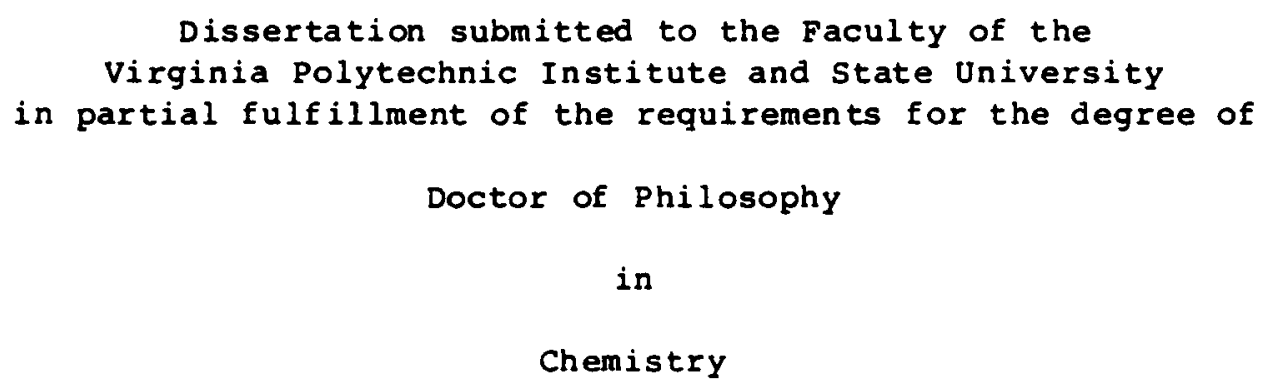

Approved :

H. M. Bell

L. T. Taylor

T.G. Mason

J.P. Wifritman

August, 1986

Blacksburg, Virginia 


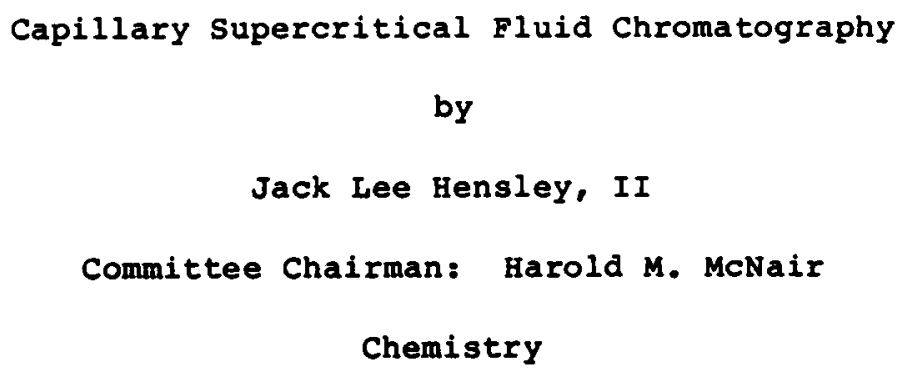




\section{ACKNOWLEDGMENTS}

The completion of this work has been assisted by many people. Their support, both personally and professionally, has been invaluable. Although these people are too numerous to receive individual mention in the limited space available, their contributions have been greatly appreciated. Among the many friends and colleagues who have been invaluable, I would like to give special mention to a few.

A special thanks is due to my research advisor, Dr. Harold McNair. The opportunities and assistance he has provided over the last few years have made an immeasurable contribution to this work. In addition to professional guidance and assistance, he has provided an excellent example of a scientist and educator.

A special thanks is due also to the Chromatography Research Group. The exchange of ideas and renewal of enthusiasm such a group generates must be experienced to be appreciated. I would particularly like to acknowledge the assistance of $\quad$ who introduced me to the finer points of capillary GC and was a great resource and colleague, and the help of , who

contributed to this work in their own individual ways. and were valued friends who made sure I was where I needed to be, and that all the paperwork got done. Without their friendship, my years at $\mathrm{Tech}$ would not have been as happy.

I would like to acknowledge the financial support of Combustion Engineering for providing the research grant for this project and the technical assistance of 
cheering me up when I was down and patiently waiting for me. I hope the coming years will be happy ones for both of us. Finally, last but most certainly not least, I would like to thank my parents. Their love, guidance and support have made possible anything which I might achieve. Without them, I would not appreciate the value of dedication and the satisfaction of achievement. To them, I respectfully dedicate this work. 
TABLE OF CONTENTS

Chapter

Page

ACKNOWLEDGEMENTS - . . . . . . . . . . . . . . . . . . .

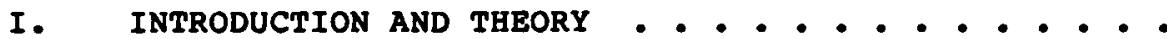

Introduction $\bullet^{-} \cdot \bullet^{-} \cdot \bullet^{-} \cdot \bullet^{-} \cdot \bullet^{-} \cdot \bullet^{-} \cdot \bullet^{-}$

The Domain of SFC . . . . . . . . . . . . . High Performance Liquid Chromatography . . . Gas Chromatography . . . . . . . . . . . Supercritical Fluid Chromatography . . . . . Theory of SFC . . . . . . . . . . . . . . Resolution of Open Tubluar Columns . . . Mobile Phases for SFC . . . . . . . . . Solubility and Pressure Programming . . . . Effects of Mixed Mobile Phases and Modifiers . - . . . . . . . . . Structure/Solubility Relationships in SFC

II. HISTORICAL . . . . . . . . . . . . . . . .

The Use of supercritical Fluids for

Extraction .................. 35

Early SFC . . . . . . . . . . . . . 36

Modern Developments in SFC . . . . . . . . 38

III. INSTRUMENTATION FOR CAPILIARY SFC . . . . . . . . . 55

Introductió .. . . . . . . . . . . . . 55

Mobile Phase .. . . . . . . . . . . . 55

Pump . . . . . . . . . . . . . . . . . . 58

Injection System . . . . . . . . . . . 63

Column . . . . . . . . . . . . . . . . . 73

Column Detector Interface . . . . . . . . 77

Detector . . . . . . . . . . . . . . 81

IV. CAPILLARY SFC COLUMN PREPARATION, COATING AND

EVALUATION . . . . . . . . . . . . . . . . . 90

Introduction .................. 90

Column Materials . . . . . . . . . . . . 90

Surface Characteristics of Fused Silica . . . 96

Capillary Column Surface Preparation . . . . 101

Suface Evaluation . . . . . . . . . . . 112

Capillary Column Coating . . . . . . . . . 114

Dynamic Coating Techniques . . . . . . 115

Static Coating Techniques .. . . . . . 116

Column Crosslinking . . . . . . . . . . 117

Capillary Column Evaluation . . . . . . . 120 
TABLE OF CONTENTS

(Continued)

Chapter

page

V. EXPERIMENTAL

Introduction . . . . . . . . . . . . . .

Materials and Reagents . . . . . . . . . .

Experimental Aspects of Column Coating . . .

Coating Solutions .. . . . . . . . . . 129

Column Filling . . . . . . . . . . . 131

Column End Sealing . . . . . . . . . . 134

Evaluation of Crosslinking Reagents for

SFC with $\mathrm{CO}_{2}$. . . . . . . . . . . . . 135

Crosslinking of ov-1701 . . . . . . . . 136

Evaluation of a Commercial column

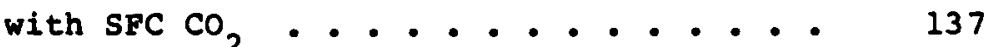

Evaluation of Polyethylene Glycol

Deactivation .. . . . . . . . . . . 137

Reproducibility of Capillary SFC . . . . . . 139

Column Activation and Deactivation . . . 139

Surface Energy Measurements . . . . . . 141

Coating and Evaluation Procedure for

Reproducibility Study . . . . . . . . 142

VI. RESULTS AND DISCUSSION

Introduction ............... 146

Evaluation of Crosslinking ......... 146

Results of Evaluation of a Commercial

Column with SCF $\mathrm{CO}_{2}$............. 153

Results of Polyethylene Glycol

Deactivation . . . . . . . . . . . . 157

Kovat's Retention Index ........... 161

Reproducibility Aspects of Capillary SFC . . . 164

VII. CONCLUSIONS

Instrumentation . . . . . . . . . 179

Injection Technique ........... 179

Polyethylene Glycol Deactivation . . . . . 180

Reproducibility of SFC .......... 181

Future พork ............... 182

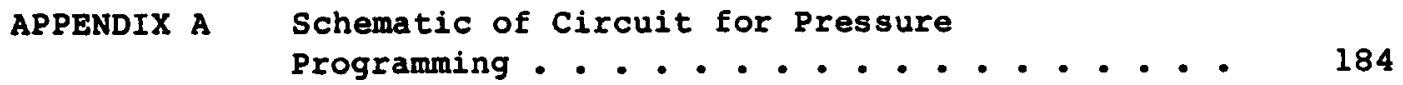

APPENDIX B Applications . . . . . . . . . . . 185

RERERENCES ....................... 198

vITA ........................ 210

vi 


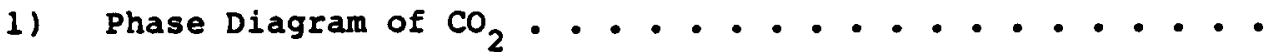

2) Paraffins from Gulfwax at $100^{\circ} \mathrm{C}$. . . . . . . . . . .

3) Dimethyl silicones showing The Effect of an Initial Isobaric Period . . . . . . . . . . . . . .

4) Dependence of Solubility Parameter $\delta$ on Reduced

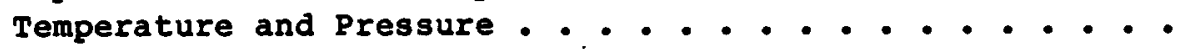

5) Selectivity $\alpha$ as a Function of Densition . . . . . . . . 30

6) Schematic of a SFC Equipped with a FID . . . . . . . . . 56

7) Schematic of a SFC Syringe Pump . . . . . . . . . . . 60

8) External Cooling of a SFC syringe Pump . . . . . . . . . . 61

9) SFC Sample Inlet Splitter . . . . . . . . . . . . . 65

10) Internal Sample Loop Value . . . . . . . . . . . . . . 66

11) Installation of Column for Direct Injection . . . . . . 68

12) Comparison of Split and Direct Injection . . . . . . . . 69

13) One $0.1 \mu L$ Injection of a 50 ppm C-24 Alkane . . . . . . 71

14) Five Consecutive $0.1 \mathrm{\mu L}$ Injections of a 10 ppr C-24 Alkane . . . . . . . . . . . . . . .

15) Five $0.1 \mu \mathrm{L} 10$ ppm Injections without Concentration on-column . . . . . . . . . . . . . . . 74

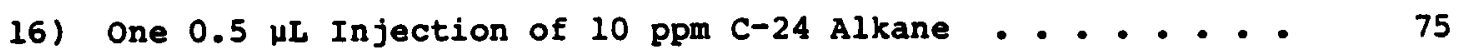

17) Cross-Section of a Capillary SFC Column . . . . . . . . . 76

18) A Zero Dead Volume SFC Detector Fitting . . . . . . . . . 79

19) SFC/FID Linear Restrictor . . . . . . . . . . . . . . 80

20) Experimental Determination of SFC/FID Linearity . . . . . . . . . . . . . . . . . . . . 


\section{LIST OF FIGURES}

(Continued)

page

21) An Overloaded Sample Component on SFC . . . . . . . . . . 85

22) Effect of $\mathrm{B}_{2}$ Gas Pressure on FID Linearity - • • • • • • • 86

23) Effect on Air pressure on FID Linearity . • . . . . . . . . 88

24) Minimum Detectable Quantity (0.5 ng) . . . . . . . . . 89

25) Structure of Fused Silica . . . . . . . . . . . . . . 93

26) Hydration and Dehydration of a silica Surface • • • • • . 99

27) Contact Angle $(\theta)$ of a Liquid Drop on a Solid surface.. .102

28) Graphical Depiction of Young's Equation . . . . . . . . . 104

29) A Proposed Mechanism for Polyethylene
Glycol Pyrolysis Deactivation . . . . . . . . . . . . . . 109

30) Mechanism of High Temperature Cyclic Deactivation . • • • 111

31) Mechanism of Free Radical Crosslinking . . . . . . . . 118

32) Example of Grob Test Mixture . . . . . . . . . . . . 123

33) Capillary Coating Apparatus . . . . . . . . . . . . . 128

34) Capillay Coating Rig for Rinsing and Filling Columns • • 132

35) High Pressure Vessel for Rinsing and Filling Columns • • • 133

36) Example of Set Up for Capillary Rise Measurement • • • • 143

37) Zisman Plot of MeOH/H $\mathrm{O}$ Mixtures on Cyclic
Deactivated Fused Silica . . . . . . . . . . . . . . . . 144

38) Initial Grob Test Mix on DCP Crosslinked
ov-l70l Column .................... . . . . . 147

39) Initial Grob Test Mix on an Uncrosslinked
ov-1701 Column ................. . . . . . . . 148

40) Grob Test Mix on DCP Crosslinked ov-1701

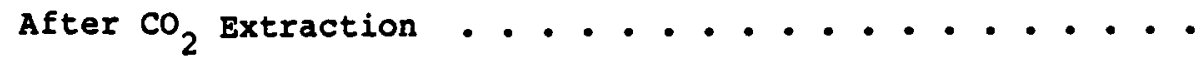




\section{LIST OF FIGURES}

(Continued)

41) Initial Grob Test Mix on ATB Crosslinked

Page

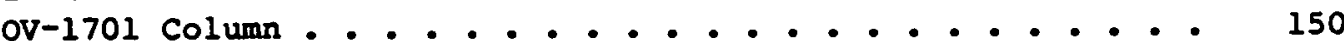

42) Grob Test Mix on ATB Crosslinked OV-1701

Column After $\mathrm{CO}_{2}$ Extraction . . . . . . . . . . 151

43) Initial Grob Test Mix on Commercial Column . . . . . . . 155

44) Grob Test Mix After $\mathrm{CO}_{2}$ Extraction of

Commercial Column .................. 156

45) Initial Grob Test Mix on Undeactivated HB-1 Column . . . . 158

46) Initial Grob Test Mix on CW Deactivated $\mathrm{HB}-1$ Column . . . 159

47) Grob Test on Undeactivated HB-I Column

After $\mathrm{CO}_{2}$ Extraction ................. 162

48) Grob Test on CW Deactivated HB-1 Column

After $\mathrm{CO}_{2}$ Extraction .................. 163

49) Paraffins by SFC on an Undeactivated column . . . . . . 169

50) Alcohols by SFC on an Undeactivated column . . . . . . . 170

51) Acids by SFC on an Undeactivated Column . . . . . . 171

52) Acids by SFC on a Superox 20M Deactivated Column . . . . . 176

53) Acids by SFC on a Mixed Cyclic Deactivated Column . . . 177

54) Effect of Flow Rate on Efficiency . . . . . . . . 187

55) SFC Separation of Triton X-100 Non-ionic Surfactant . . . 188

56) SF-96, Dimethylpolysiloxane Fluid, by SFC . . . . . . 190

57) ov-235, GC stationary Phase, by SFC . . . . . . . . 191

58) Liquid $\mathrm{CO}_{2}$ Extract of Bay Leaves by SFC . . . . . . . . 194

59) Liquid $\mathrm{CO}_{2}$ Extract of Basil by SFC . . . . . . . . 195

60) Liquid $\mathrm{CO}_{2}$ Extract of oregano by SFC . . . . . . . . 196 
1) Chromatographic Physical Properties of Gases, Liquids and supercritical Fluids . . . . . . . . . . .

2) Useful supercritical Fluids for SFC . . . . . . . . . . .

3) Supercritical Fluids with Limited Utility for SFC . . • • 16

4) Composition of Sodium and Borosilicate Glasses . . . . . . 91

5) Concentrations of Impurities in Various Fused silicas . . . 96

6) Surface Hydroxyl Concentrations on Glass and Fused Silica . . . . . . . . . . . . . . . . . . 100

7) Surface Tensions of Various Liquid Phases . . . . . . . . 105

8) Surface Tensions and Densities for Methanol $/ \mathrm{H}_{2} \mathrm{O}$ Mixtures . . . . . . . . . . . . . .

9) Decomposition Temperatures of Various Crosslinking Initiators . . . . . . . . . . . . . . .

10) Analyte Concentration in Grob Test Mixture II . . . . . .

11) Effect of Temperature Changes During Column Coating . . . 130

12) Results of Crosslinking ov-1701 with ATB or DCP . . . . . 152

13) Evaluation of Crosslink Efficiency of a Commercial

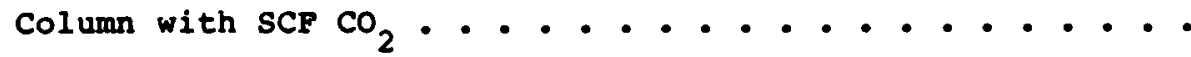

14) Comparison of CW Deactivated HB-1 Column to an Undeactivated Column Before and After $\mathrm{CO}_{2}$ Extraction . -

15) Kovat's Retention Indices for HB-1 showing Influence of Deactivation Layer . . . . . . . . . . . . . .

16) Relative Standard Deviations of Peak Areas for Paraffins . . . . . . . . . . . . . . . . . . .

17) Relative Standard Deviations of Peak Areas for Alcohols . . . . . . . . . . . . . . . . . . .

18) Relative Standard Deviations of Peak Areas for Acids . . 
CHAPTER I

\section{INTRODUCTION AND THEORY}

Supercritical Fluid Chromatography (SFC) is a technique which has been given a great deal of attention over the last few years. Although supercritical fluids have been investigated for over a hundred years and chromatography with a supercritical fluid was first described in $1962^{l}$, the emergence of SFC as a practical analytical technique is a very recent phenomenon. The early work demonstrating the feasibility of SFC was severely limited by technological, instrumental and material constraints. In addition, the rapid emergence and development of HPLC and capillary GC depleted the interest that was initially expressed in SFC. However, the SFC technique did not disappear. Steady, but slow progress was made throughout the two decades following its introduction. As advances in GC and HPLC technology were made, they were applied to SFC. Capillary SFC is a technique which is still in its infancy. First reported in the literature in $1981^{2}$, capillary SFC was definitely a technique limited to laboratories involved with "state-of-the-art" chromatography research. The success of the SFC research is evident in the fact that in March of 1986 four commercial capillary SFC instruments were introduced where none had previously been commercially available. This availability of instrumentation should generate increased interest in the area and allow the application of SFC to a wider range of samples and applications.

The objective of this work was to develop a capillary chromatographic system capable of being utilized for SFC. After 
the development and testing of this instrument, it was used to develop and test fused silica capillary columns made specifically for SFC applications. The columns were developed to be inactive to sample adsorption and capable of maintaining their efficiency under SFC conditions. A combination of column pre-treatments, deactivation steps, stable coating methods and efficient cross-linking of the polymeric stationary phase was needed to meet this goal. After the development and evaluation of these analytical columns, they were then used to develop and extend the applications of supercritical fluid chromatography.

\section{The Domain of Supercritical Fluid Chromatography}

Supercritical Fluid Chromatography (SFC) is under development to address specific problems in separation science that are not easily resolved by either High Performance Liquid Chromatography (HPLC) or Gas Chromatography (GC). SFC is not intended or designed to replace either of these techniques. It is, however, designed to supplement GC and HPLC in areas where analysis by these methods is either impossible or difficult due to incompatibility of the sample and the analysis technique. In order to discuss the usefulness and potential of SFC it is first necessary to examine HPLC and GC to determine their appropriate areas of application.

\section{High Performance Liguid Chromatography}

High Performance Iiquid Chromatography utilizes a column packed with small (3-10 micron) particles which act as a stationary phase for the separation of analytes which pass through the column as a solute 
in a liquid medium. The interaction of analytes with either the surface or the solvated layer of the solid particles results in differential retention of analytes and their separation as they migrate down the column. HPLC is a highly selective technique due to two forms of selective interaction. The first form is the selective interaction of the sample and the stationary phase. The choice of chemically bonded functionalities such as alkyl, phenyl or cyanopropyl groups which are " bonded to silica gel provide a means for increasing the selectivity and therefore the resolution of the chromatographic separation. The second form of interaction is between the sample and the mobile phase. The mobile phase in the case of BPLC is a liquid in which the sample is transported through the column. The effect of analyte solubility in the mobile phase has a definite effect on the separation. If a sample is very soluble in the mobile phase there is little to no interaction with the stationary phase and no retention or separation occurs. If the sample has a great deal of interaction with the stationary phase and very little solubility in the mobile phase the sample will remain on the column and will not elute or will not elute within a reasonable time. For this reason the technique of gradient elution, involving the change from a weak solvent to a strong solvent during the analytical run, was developed to push the strongly held analytes from the column. Modifying selectivity by changing the stationary or the mobile phase imparts a great range of separating ability to the HPLC technique. The range of samples amenable to HPLC is large and is limited only by the ability to dissolve the sample in a reasonable solvent. However, 
4

the technique is inherently limited by the use of a packed column. The packed column is needed because of the low diffusivity of liquids. It is important that a reasonably fast equilibria exists between the sample in the mobile phase and the sample associated with the stationary phase. In liquids this means that thin films for rapid mass transfer and close asociation of particles should exist in the column to minimize the transport time in the liquid phase. On a practical scale this leads to very small particles very tightly packed. Some work has been performed on open tubular columns but the use of conventional liquid mobile phases leads to very small column diameters (3-5 micron), which yields an extremely low sample capacity and demands sample detection and instrumental requirements beyond present day technology. ${ }^{3}$ The limitation of the packed column is one of efficiency and not of selectivity. The efficiency, $\mathrm{N}$, as measured by theoretical plates is a function of column length and can in the case of packed columns. be increased by either decreasing particle size (dp) or increasing column length, $I$, according to the following equation. 4

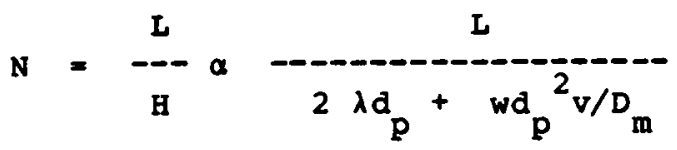

Since either of these methods increases the pressure necessary to push a reasonable flow through the column, a practical limitation exists in the instrumentation required. A pressure of 5000 p.s.i. is commonly accepted as a reasonable high pressure limit for pumps and injection systems in terms of chromatographic efficiency and cost. This limit is 
5

very quickly approached with two $25 \mathrm{~cm}$ high performance columns connected in series and provides a very real limit on absolute efficiency. Another limitation of HPLC involves the detection systems available. The most commonly used HPLC detectors are ultra-violet, fluorescence and refractive index detectors. The uv and fluoresence detectors are sensitive, but highly selective and discriminatory in response while the $R I$ detector is universal in response but insensitive. Gas Chromatography

Gas Chromatography was revolutionized by the introduction of capillary open tubular columns by Golay in $1957 . .^{5}$ The high diffusivity of gases permits the use of open tubular columns and this open structure has such a low pressure drop that very long lengths (50-100 meters) are able to be utilized. These long column lengths give high plate counts and therefore a high degree of resolution. Capillary GC is not, however, as selective as HPLC. The selectivity of GC is a function of interactions between the sample and the stationary phase. However, the interaction of the sample and the mobile phase is not generally a factor in the GC separation. The gaseous mobile phase is used merely as a transport system and may have a small effect on efficiency due to its physical characteristics, but it does not selectively interact with the sample. This loss of selective interaction is more than offset by the increase in column efficiency imparted by the open tubular column.

The detection systems available for GC encompass a wide range of selective and sensitive detectors, but the most widely used is the flame ionization detector which has good sensitivity $\left(10^{-11} \mathrm{~g}\right)$ for organic 
compounds. 6 The gas chromatograph is limited in terms of sample handling ability due to a restriction that the sample must be volatile and thermally stable in order to be analyzed. Gas chromatographs can operate up to $350^{\circ} \mathrm{C}$ with high temperature stationary phase. Therefore a sample must have a reasonable vapor pressure at this temperature in order to move through the column. In addition, the sample should not decompose at the temperature of operation. High temperatures generate decomposition products which detrimentally affect the analysis. These restrictions limit GC from being used with many high molecular weight compounds due to their non-volatility and exclude many complex molecules such as biochemicals and pharmaceuticals due to their lack of thermal stability.

Supercritical Fluid Chromatography

Gas Chromatography and High Performance Liquid Chromatography both do many samples well, but some samples are not well addressed by either technique. These samples are fairly high in molecular weight (1000 to 10,000 a.m.u.) but are not sufficiently volatile nor thermally stable enough for GC, and need detectors or efficiency (number of plates) which HPLC can not provide. Supercritical Fluid Chromatography is being developed in an attempt to address the need for an analysis technique that provides high efficiency capillary separation of non-volatile, thermally labile compounds.

Supercritical fluids are used in chromatography because of their physical properties that incorporate elements of both HPLC and GC. A supercritical fluid is a highly compressed gas which exists at or above 
its critical point. The critical point is the temperature and pressure combination where the physical properties of the gas and liquid phases are the same. ${ }^{7}$ Above this critical temperature the kinetic energy of the gas is so high that compression to a liquid state is not possible (Figure 1). The result is a gaseous state which has an appreciable density. This supercritical fluid has properties of both gases and liquids. Table 1 shows a comparison of the properties of gases, liquids and supercritical fluids in terms of density, viscosity and diffusivity. The chromatographic advantages of SFC are based on these properties. The relatively high density gives the supercritical fluid the ability to dissolve samples so that transport in the mobile phase can be accomplished without the need to volatilize the sample into the gas phase. Since the solvating ability of a supercritical fluid is proportional to its density, and since the density is a function of the temperature and pressure, a high pressure drop across the column during the analysis would mean that the sample would pass from a region where it would be strongly solvated into a region of less solubility. ${ }^{8}$ This situation would lead to chromatographic broadening of elution bands and loss of efficiency.

With the low viscosity of supercritical fluids this effect is minimized. The low viscosity allows the use of reasonable flow rates without the high pressure drops involved with more viscous fluids. As a result of the low viscosity of supercritical fluids, high sample diffusion coefficients are produced. These high diffusion coefficients permit the use of faster flow rates since equilibrium across the column is faster. 
8

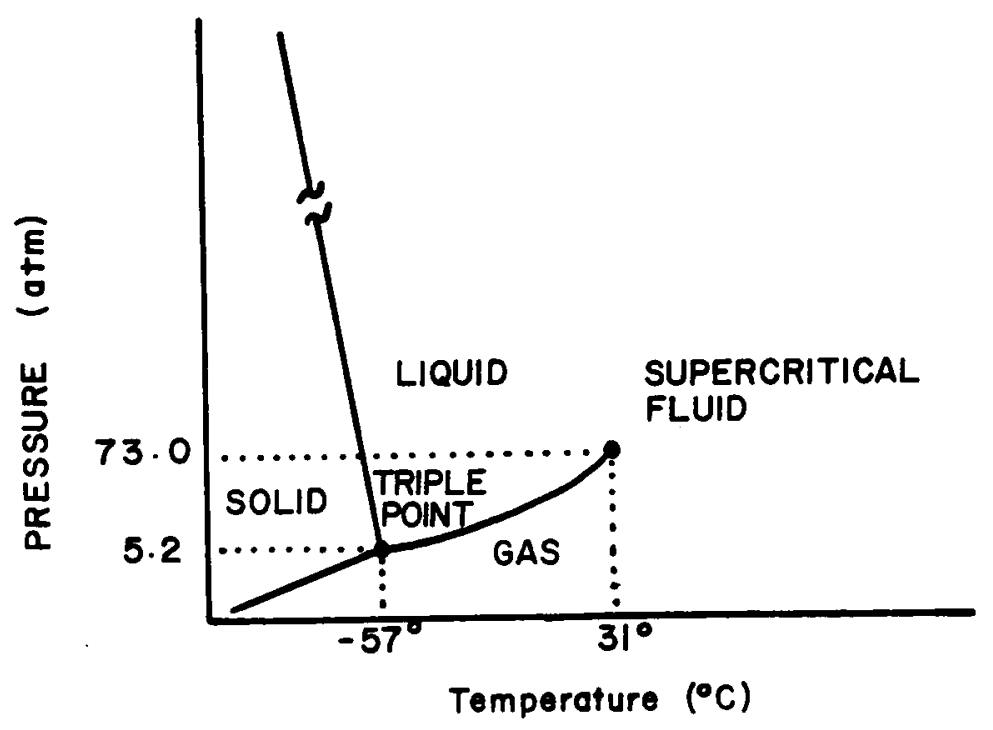

Figure 1. Phase diagram of $\mathrm{CO}_{2}$ showing the supercritical region. 
Table 1. Chromatographic Physical Properties of Gases, Liquids and Supercritical Fluids

\section{Phase}

gas at $21^{\circ} \mathrm{C}, 1$ atm.

supercritical fluid

liquid

$$
\text { Density }
$$
$\left(\mathrm{g} / \mathrm{cm}^{3}\right)$

$10^{-3}$

$0.3-0.8$

1
Diffusion

$\underline{(\mathrm{cm} 2} 2 \mathrm{sec})$

$$
10^{-1}
$$

$10^{-3}-10^{-4}$

$<10^{-5}$ viscosity

$(\mathrm{g} / \mathrm{cm}-\mathrm{sec})$

$10^{-4}$

$10^{-4}-10^{-3}$

$10-^{2}$ 
The high diffusivity of supercritical fluids allows the use of open tubular columns of practical internal diameters $(30-100 \mu \mathrm{m} i . d$.$) because$ the sample approaches equilibrium in the mobile phase and thus minimizes the band broadening caused by classical laminar flow. In capillary HPLC, the slow diffusion in liquids dictates that column internal diameter should be on the order of 5-10 microns. The higher diffusivities of supercritical fluids permits the use of larger column capacity diameters (30-100 microns) and the accompanying increase in sample. This permits the use of a wide variety of conventional chromatographic detectors.

Theory of SFC

Resolution of Open Tubular Columns

A long open tube coated with a uniform thin film has been characterized by Golay in terms of the height of the theoretical plate (в). 9

$\mathrm{H}=\frac{2 \mathrm{D}_{\mathrm{m}}}{\mathrm{u}}+\frac{\left(1+6 \mathrm{k}^{\prime}+11 \mathrm{k}^{\prime}\right) \mathrm{d}_{\mathrm{c}}{ }^{2} \mathrm{u}}{96\left(1+\mathrm{k}^{\prime}\right)^{2} \mathrm{D}_{\mathrm{m}}}+\frac{2 \mathrm{k}^{\prime} \mathrm{d}_{\mathrm{f}}{ }^{2} \mathrm{u}}{3\left(1+\mathrm{k}^{\prime}\right)^{2} \mathrm{D}_{\mathrm{s}}}$

$D_{m}=$ diffusion coefficient of the solute in the mobile phase

$d_{f}=f i l m$ thickness of the stationary phase

$D_{s}=$ diffusion coefficient of the solute in the stationary phase

$d_{c}=$ column diameter

$\mathrm{u}=$ mobile phase linear velocity

$k^{\prime}$ = capacity factor 
11

This equation is important to chromatography because $H=I / N$ where $\mathrm{I}$ is the length of the column and $\mathrm{N}$ is the number of theoretical plates. The number of theoretical plates is related to the ability to resolve two components by the master resolution equation:

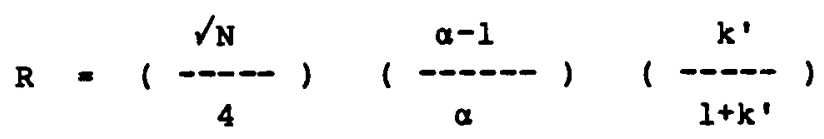

where $R$ - resolution between two components. 10

$$
\begin{aligned}
& \alpha=\text { selectivity } \\
& \mathbf{k}^{\prime}=\text { capacity factor } \\
& N^{\prime}=\text { number of theoretical plates }
\end{aligned}
$$

To increase the resolution between two components it is necessary to increase either the capacity factor ( $\left.k^{\prime}\right)$, the selectivity (a), or the efficiency $(N)$ of the system. In order to increase $N$ it is necessary to either increase $\mathrm{L}$ since $\mathrm{N}$ is proportional to $\mathrm{L}$ or to decrease $\mathrm{H}$ by use of the Golay equation. One way to minimize $\mathrm{H}$ is by decreasing the diameter of the column and by maintaining column flow velocity at an optimum value. The length of the column should only be increased to the minimum needed to achieve acceptable resolution since the analysis time is directly proportional to column length. The time needed to perform an analysis can be represented by ${ }^{11}$

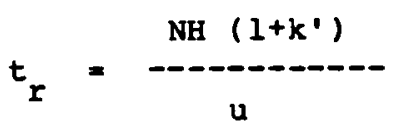


In order to achieve fast analysis times a value for the linear velocity $u$ is chosen that is larger than optimum. As the flow rate increases the $\mathrm{H} / \mathrm{u}$ term approaches the value Hmin/2u opt where Hmin is the minimum theoretical plate height on a plot of $\mathrm{H}$ vs. $u$ and $u_{\text {opt }}$ is the linear velocity of mobile phase corresponding to that minimum. When $k^{\prime}$ values are chosen between 1 and 5 the value of $\mathrm{Hmin} / 2 \mathrm{u}_{\mathrm{opt}}$ is approximately $0.6 \mathrm{~d}_{\mathrm{c}}{ }^{2} / 6 \mathrm{Dm}$. Under the same conditions an efficient packed column has a value of $2 d_{p}{ }^{2} / 3 D_{m}$. For a supercritical mobile phase where $D_{m}$ is the same for both packed and capillary columns this means that equal resolution per unit time is achieved at $d_{c}=2.6 d_{p}$. Therefore in order to achieve the same resolution per unit time as a $10 \mu \mathrm{m}$ particle packed column a $26 \mu \mathrm{m}$ capillary must be used. The problem is complicated, however, by the fact that at low pressures a significant decrease in density will occur across the packed column as a function of the pressure drop. This will lead to a decrease in resolution at lower pressures for the packed column while at higher pressures the density drop is minimized relative to the density of the column. This means that at high pressures the packed column will excel while at low pressures the capillary will have the advantage.

If a value of $10^{-5} \mathrm{~cm}^{2} / \mathrm{sec}$ is assumed for the diffusion coefficient of the liquid and $5 \times 10^{-4}$ for the supercritical fluid then using the above equations and approximations a value of $18 d_{p}$ is equivalent to $d_{c}$. Therefore a 5 um HPLC packed column gives the same resolution per unit time as a $90 \mu \mathrm{m}$ capillary SFC column. A $10 \mu \mathrm{m}$ particle HPLC column is then equivalent to a $180 \mu \mathrm{m}$ i.d. SFC capillary. 
Since diffusion in gases is much faster than in supercritical fluids the very small diameter columns needed for SFC is prohibitive in terms of sample capacity and detectability of components. Assuming diffusion in a gas to be $0.1 \mathrm{~cm}^{2} / \mathrm{sec}$, then an SFC column must be $1 / 14$ th the size of an equivalent GC column. A $250 \mu \mathrm{m}$ capillary GC column would be equivalent in terms of resolution per time to an $18 \mu \mathrm{m} i . d$. SFC column while a $100 \mu \mathrm{m} \mathrm{GC}$ column would compare to a $7 \mu \mathrm{m}$ SFC column. At these diameters SFC columns are no longer practical with currently available instrumentation. This means that capillary GC will always have an advantage in speed of analysis and should be used on samples wherever it is an appropriate technique. Mobile Phases for SFC

Many gases and liquids have been used as supercritical fluids for chromatography. Theoretically any substances which exists above its critical temperature without decomposition can be used as a supercritical fluid in chromatography, but practical considerations limit the possibilities. A supercritical fluid should meet several criteria in order to be a useful mobile phase. The supercritical fluid range of the substance should be accessible with available instrumentation. The pressure limits of available commercial instrumentation are about 5000 p.s.i. with some models offering limits up to $10,000 \mathrm{p.s.i.} \mathrm{The} \mathrm{practical} \mathrm{limit} \mathrm{on} \mathrm{commercial} \mathrm{instruments} \mathrm{is}$ the pressure limits connections, tubing and injection valves impose on the system. Since one of the advantages of supercritical fluid chromatography is the low thermal stress imposed on the sample, it is 
14

necessary that the critical temperature of the fluid be well below the decomposition temperature of the analyte. Table 2 shows critical temperature and pressure data on some of the substances which have been used or proposed as SFC mobile phases.

The most important parameter chromatographically is the ability of the supercritical fluid to dissolve the sample. A good rule of thumb is the observation that "like dissolves like" which indicates the use of polar solvents for polar samples and nonpolar solvents for nonpolar samples. Unfortunately, the more polar gases and common liquid solvents have such strong intermolecular attractions that critical temperatures and pressures are quite high. Table 3 contains critical data on gases and liquids that have high critical temperatures and pressures, or are not practical due to safety or cost considerations.

Carbon dioxide is the most widely used supercritical fluid for a wide variety of reasons. A critical temperature of $31^{\circ} \mathrm{C}$ and a critical pressure of $73 \mathrm{~atm}$ allows its use without too much regard to special instrumental considerations. ${ }^{12}$ A very low toxicity and lack of flammability make it a convenient and safe solvent especially in a hazardous or industrial environment. The low cost of $\mathrm{CO}_{2}$ makes it an economical solvent even when it is purchased in a high purity grade. Carbon dioxide is an excellent solvent for many materials. It has been used extensively in industry for extraction processes due to its solvating ability. ${ }^{13-16}$ Although $\mathrm{CO}_{2}$ is non-polar, the electron density associated with the $\mathrm{C}-\mathrm{O}$ double bond makes $\mathrm{CO}_{2}$ a better solvent for many 
Table 2. Useful Supercritical Fluids

\begin{tabular}{|c|c|c|}
\hline Compound & $\operatorname{Tc}\left({ }^{\circ} \mathrm{C}\right)$ & Pc (atm) \\
\hline $\mathrm{CO}_{2}$ & 31.3 & 72.9 \\
\hline $\mathrm{NH}_{3}$ & 132.3 & 111.3 \\
\hline ethane & 32.4 & 48.3 \\
\hline n-propane & 96.8 & 42.0 \\
\hline n-butane & 152.0 & 37.5 \\
\hline n-pentane & 196.6 & 33.3 \\
\hline $\mathrm{CCl}_{2} \mathrm{~F}_{2}$ & 111.7 & 39.4 \\
\hline $\mathrm{CCl}_{2} \mathrm{HF}$ & 178.5 & 51.0 \\
\hline $\mathrm{CCl}_{3} \mathrm{~F}$ & 196.6 & 41.7 \\
\hline $\mathrm{N}_{2} \mathrm{O}$ & 36.5 & 71.4 \\
\hline diethyl ether & 193.6 & 36.3 \\
\hline dichlorotetrafluoroethane & 146.7 & 35.5 \\
\hline sulphur hexafluoride & 45.6 & 37.1 \\
\hline $\mathrm{CHF}_{3}$ & 23.0 & 47.6 \\
\hline $\mathrm{CH}_{3} \mathrm{~F}$ & 45 & 58 \\
\hline
\end{tabular}


Table 3. Supercritical Fluids with Limited Utility

\begin{tabular}{lcc} 
Compound & $\underline{T C\left({ }^{\circ} \mathrm{C}\right)}$ & $\underline{P C(\mathrm{~atm})}$ \\
\hline $\mathrm{H}_{2} \mathrm{O}$ & 374.4 & 226.8 \\
methanol & 240.5 & 78.9 \\
ethanol & 243.4 & 63.0 \\
isopropanol & 253.3 & 47.0 \\
n-hexane & 234.2 & 29.6 \\
2,3 -dimethyl butane & 226.8 & 31.0 \\
benzene & 288.9 & 48.3 \\
xenon & 16.6 & 58.0 \\
krypton & -64 & 54 \\
so 2 & 158 & 78 \\
CS & 279 & 78 \\
H $_{2} \mathrm{~S}$ & 89 & 100
\end{tabular}


samples than would normally be expected with a strictly non-polar solvent. In general $\mathrm{CO}_{2}$ behaves like a solvent with a solvating power slightly greater than hexane. 17

A further advantage of $\mathrm{CO}_{2}$ is its compatibility with a wide range of detection systems. Carbon dioxide is not oxidizable which allows the use of a flame ionization detector (FID) or nitrogen phosphorous detector (NPD). Large spectral widows due to the simple structure of $\mathrm{CO}_{2}$ allow the use of spectroscopic detectors in the ultraviolet, infrared and visible regions. $\mathrm{CO}_{2}$ has also been successfully used with mass spectroscopy to obtain both quantitative and qualitative information. Nitrous oxide $\left(\mathrm{N}_{2} \mathrm{O}\right)$ has many of the advantages of $\mathrm{CO}_{2}$ but is not as economical or as non-toxic. In addition the solvating power and selectivity of $\mathrm{N}_{2} \mathrm{O}$ as a solvent has not been shown to be sufficiently different from $\mathrm{CO}_{2}$ to justify its widespread use. 18

Ammonia $\left(\mathrm{NH}_{3}\right)$ has been used successfully in SFC, but special care is needed in providing inert, non-degradeable connections. ${ }^{19}$ Due to the toxicity of $\mathrm{NH}_{3}$ it is important to provide for adequate ventilation in the event of leakage, and most facilities are not equipped to handle a poisonous gas at 5000 p.s.i.

Pentane has been used extensively with uv and fluorescence detectors but is of course not suitable as a solvent with F.I.D.'s. Pentane has shown an increased solubility for polystyrene compared to $\mathrm{CO}_{2} \cdot 20$ Molecular weights up to 3000 have been eluted with pentane while $\mathrm{CO}_{2}$ only elutes polystyrene oligomers with a molecular weight of about 1000. Pentane has been used primarily with polyaromatic and polynuclear 
hydrocarbons since their strong uv absorption and fluorescence emission allows sensitive detection of low levels of analyte. 21

Sulphur hexafluoride has been used for petroleum analysis but its usefulness with flame ionization detectors is limited. Although $\mathrm{SF}_{6}$ has a low background in the flame, it evidently decomposes to HF gas which attacks the exposed metal of the flame tip and collection electrode. The use of sulphur hexafluoride with a F.I.D. has been reported by Brownlee. $^{22}$ The exposed metal parts in the detector were gold electroplated in this study to prevent HF attack.

Some other fluids which have been used include freon, which has been used in combination with $\mathrm{CO}_{2}$ to provide a full spectral region of transparency in FTIR spectrometers. ${ }^{23}$ Xenon has been proposed due to its very simple spectral characteristics, but it is very expensive and will probably not be economically feasible for widespread use. 24 Solubility and Pressure Programming

The solubility of a compound in a supercritical fluid has a direct relationship to the density of the fluid. ${ }^{25}$ Increasing the density increases the interaction between solute and solvent by decreasing the distance between molecules which results in a enhanced ability of the solvent to dissolve the sample. Giddings has shown that a minimum density is necessary to transport the sample through the column. ${ }^{26}$ This differs from GC where some migration is expected through the column even at lower temperatures. In Figure 2 this point is dramatically illustrated by the analysis of a paraffin wax sample performed in our laboratory. The column in both cases is the same but in the first run a 

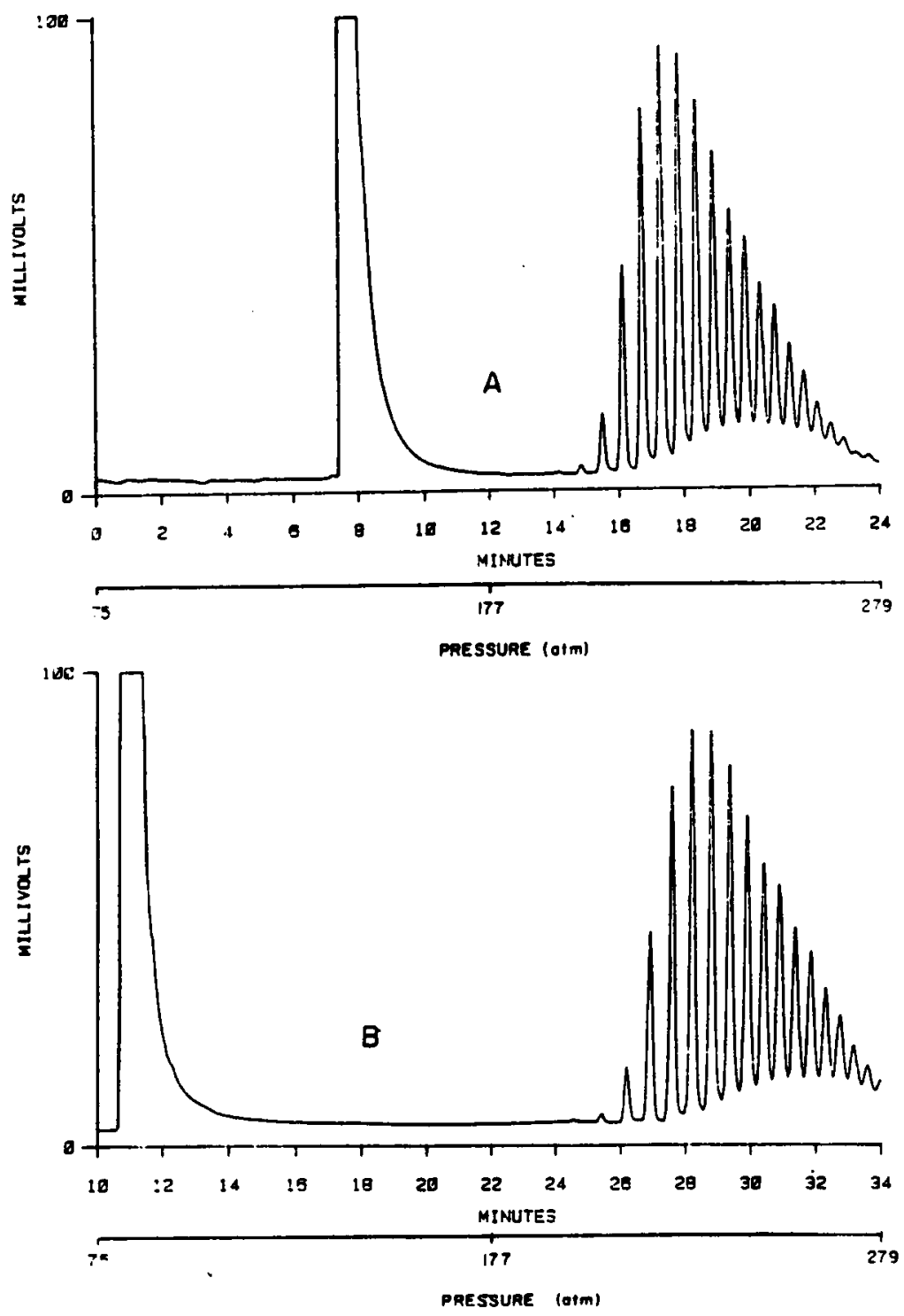

Figure 2. Paraffins from Gulfwax by SFC at $100^{\circ} \mathrm{C}$. Figure A shows immediate pressure program. Figure $B$ shows the effect of a 10 minute isobaric period. Note the similar elution pattern is not influenced by the hold period. Solvent dichloromethane. 
pressure program was initiated upon injection, while in the second case the pressure was held constant until 10 minutes had passed allowing the solvent to elute and then programming at the same rate (125 p.s.i. per minute) as the first run. It can be seen that the elution times are offset by 10 minutes and the peak profile is unchanged. All compounds are eluting at the same density regardless of the residence time in the column. The advantage of this delayed pressure program is that it opens a window of analysis for components that are more $\mathrm{CO}_{2}$ soluble and would elute with the solvent front. The separation of dimethyl silicones in Figure 3 was made in our laboratory to illustrate an example of this effect. The delay in starting the pressure ramp permits the solvent to clear the column before the early eluting components begin to emerge. These effects illustrate clearly the statement by Hannay in 1880.27

"The liquid condition of fluids has very little to do with their solvent power, but only indicates molecular closeness. Should the closeness be attained by external pressure instead of internal attraction, the result is that the same or even greater solvent power is obtained...."

The effect of increasing the pressure to increase the density of a supercritical fluid and decrease the capacity ratio for an analyte has been widely recognized. ${ }^{28-30}$ since it is the density of the mobile phase and not its pressure that is of interest, it is useful to relate the pressure change to a density change. When the pressure drop is relatively small: 

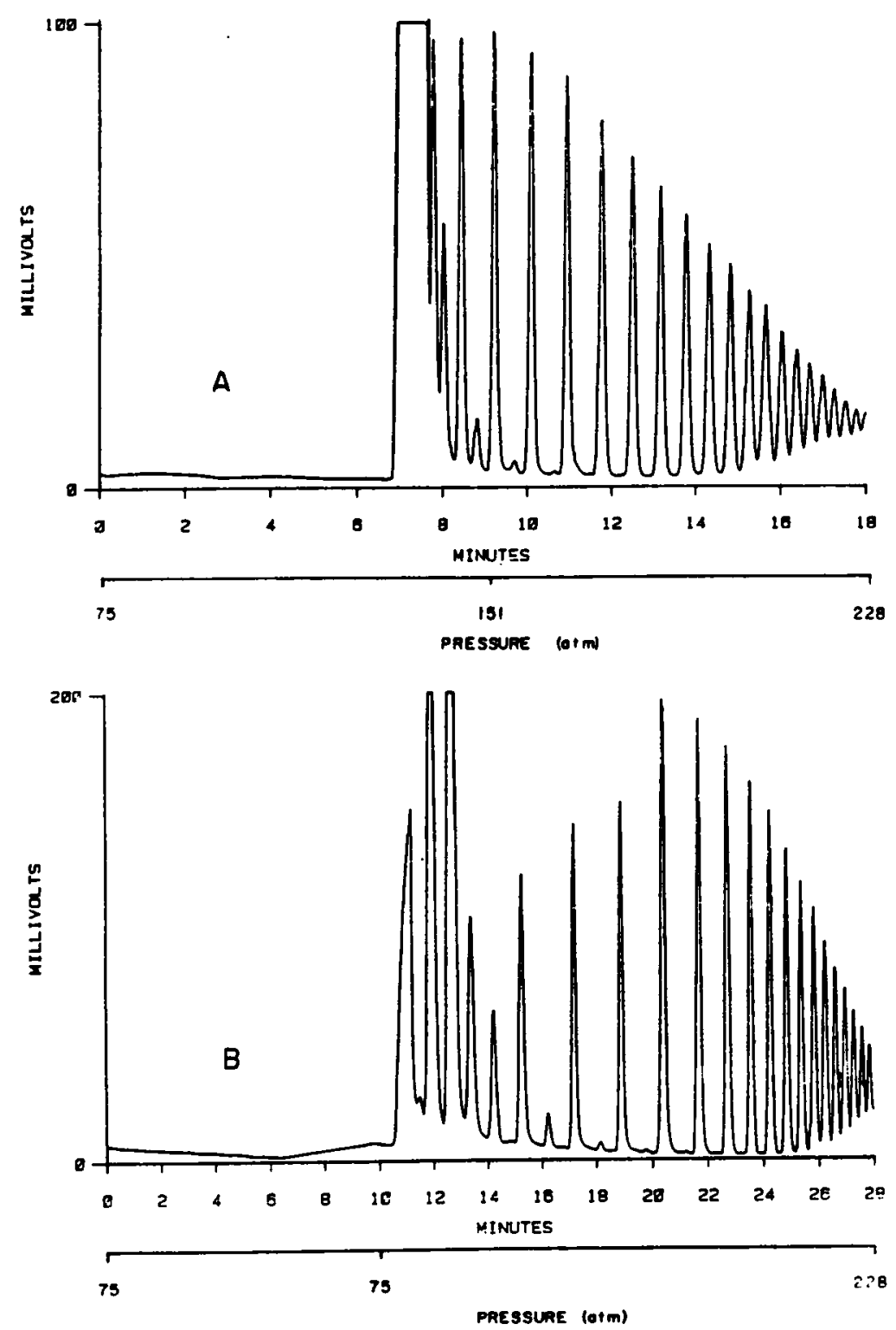

Figure 3. A commercial dimethyl silicone product by SFC at $100^{\circ} \mathrm{C}$. Figure A shows immediate pressure program. Figure B shows the effect of a 10 minute isobaric period. Note the increased retention of early eluting components. Solvent carbon disulphide. 


$$
\frac{\Delta p}{p}=S(\Delta P / P)
$$

where $p$ is the density, $P$ is the pressure and $s$ is the fractional density change per fractional change in pressure. While the values of $\mathbf{S}$ that have been encountered in SFC range from 0.2 to 6 , the most commonly encountered values for $s$ are between 0.7 and $4.4 .^{31}$ It has been reported that the pressure drop across a column is linear so the density drop can also be approximated as nearly linear. 32,33 This condition causes a chromatographic problem analogous to a negative temperature gradient in GC if the SFC run is performed at constant density. The usual method of compensating for this effect is to increase the density during the run by either increasing the pressure, decreasing the temperature or both.

Pressure programming was first performed by Jentoft and Gouw 34,35 and later used by Bartmann and Schneider 30,36 and Nieman and Rogers. 37 All of the above were linear pressure programs as a function of time.

Attempts have been made to link the solvent power of $\mathrm{CO}_{2}$ to the Hildebrand solubility parameter and to a first approximation useful information can be obtained. 25,17 However a more rigorous approach has been described by Schoenmakers ${ }^{38}$ using a 3 parameter equation of state suggested by Lee and Kesler. ${ }^{39}$ This allows the construction of a plot of solubility parameter versus reduced pressure for $\mathrm{CO}_{2}$ that is shown in Figure 4. Note the large jump in solvent power at $T_{r}=1$ and then more gradual rise at higher temperatures. This model was used to predict elution behaviors with good success. It should be noted that the 


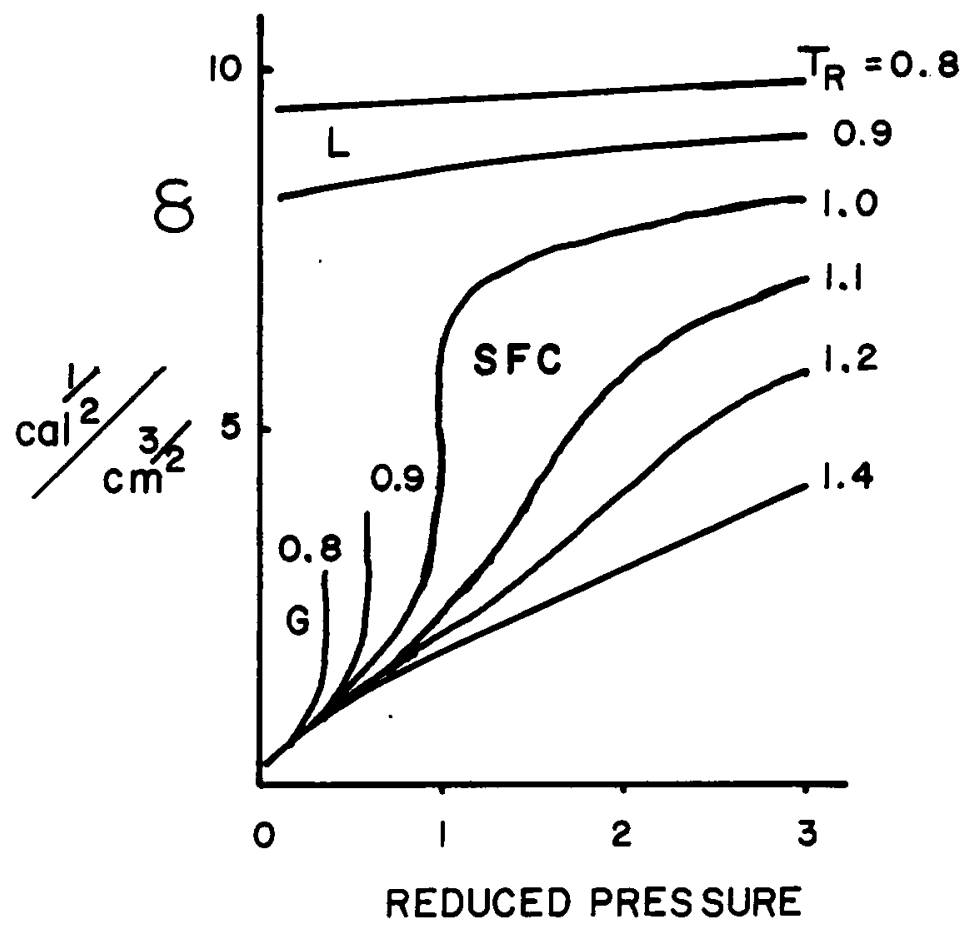

Figure 4. The dependence of solubility parameter $\delta$ on reduced temperature and reduced pressure. 
variation of retention $(\log k)$ with pressure yields complicated curves while a plot of $\log k^{\prime}$ vs. log density does not give a straight line unless a limited range of high densities is used. Selectivity decreases with increasing pressure as is shown by the similar elution times of homologs at high pressures.

A practical and thorough discussion of SFC theory based on chromatographic principles has been presented by Peaden and Lee. 40 They review the assumptions that the diffusion coefficient times the mobile phase density is a constant, and that for an ideal gas pV is a constant These assumptions have been used to show that pressure drops should not adversely affect the efficiency of SFC chromatography. 41,42 These assumptions have been found not to hold at higher pressures ${ }^{43}$, although they may be adequate up to about 808 of the critical pressure. ${ }^{32}$ These deviations from ideality occur because of the relationship of viscosity to solubility. In liquids, diffusion coefficients decrease with increasing viscosity and can become the single most important factor affecting the diffusibility of a solute. Because of the variation of viscosity with pressure, particularly in the region of the critical point ${ }^{14,44}$ the product of the diffusion coefficient and the density also varies with pressure. The variation of a diffusion coefficient along the length of the column due to changes in viscosity and density caused by the pressure drop can cause actual plate heights to be greater than those predicted from theory. Rogers has found that increased turbulent flow in the column compensates for these effects. Turbulent flow can be characterized by the Reynold's number $\left(R_{e}\right)$, a dimensionless quantity 
which is related to the fluid density "p" the velocity " $v$ ", the viscosity " $\eta$ " and the diameter of either a particle or open tube, "dp", as the case may be.

$$
R_{e}=\frac{p v d_{p}}{n}
$$

Chromatographically, turbulent flow is a positive characteristic since the alternative, laminar flow, results in band spreading of the analyte in the column. Open tubular columns require $R_{e}$ values of around 2200 for turbulent flow to occur. Supercritical fluids achieve the condition of turbulent flow more easily than either gases or liquids. ${ }^{33}$ The ratio $p / n$ is larger for supercritical fluids than gases because the density of a supercritical fluid is greater than that of a gas. The ratio $\rho / n$ is also greater for supercritical fluids compared to liquids because of the decreased viscosity of supercritical fluids at liquid-like densities. Values of $R_{e}$ for supercritical fluids are about 1000 times greater compared to liquids. The drawback to this flow profile is that diffusion in the stationary phase must be fast so that it does not slow down the equilibrium process with the center of the flow path in either the open tube or between the particles of a packed column. 45 The density change caused by a pressure drop affects not only sample solubility but also selectivity a which is defined as

$$
a=\frac{t^{\prime} R_{2}}{t^{\prime}}
$$


where the adjusted retention time $t$ 'R of compounds 1 and 2 is corrected by subtracting the time needed for elution of an unretained peak $\left(t_{0}\right)$ from the retention time $t_{R}$.

$$
t_{R}^{\prime}=t_{R}-t_{0}
$$

There are two approaches to this problem, one is to minimize the pressure drop across the column by either using larger particles (long columns, long analysis times) $)^{8}$ to diminish the pressure drop or to use an open tubular capillary column which has a low pressure drop. ${ }^{2}$ The second approach is to accept that the pressure drop will be large with small particles (3-10 microns) but work in a region where the change of density as a function of pressure, $d p / d t$, is not large (high pressures). 46

Both approaches have been found to be practical and possible although the use of large particles has all but disappeared.

The retention of a solute on an SFC column seems to be a function of three major factors. These factors are (1) the volatility of the sample, (2) the solubility of the sample in the mobile phase, and (3) specific interactions with the stationary phase. These factors allow the separation of two compounds of the same volatility and different solubilities or two compounds with the same solubility and different volatilities by appropriate choice of temperature and density. 47 In the case of non volatile species, particularly homologous series, where the interaction of sample and stationary phase is nearly identical, the primary factor influencing retention is solvent density. Plots of log 
K' vs density have been widely reported to be linear or nearly linear. $37,44,46,48$

Temperature has been shown to influence resolution greatly at higher pressures where density changes less with temperature or pressure. ${ }^{13}$ This effect is probably due to the widely known observation that increasing the temperature of the solvent usually increases the solubility of a solute in that solvent. At constant pressure an increase in temperature will increase $k^{\prime}$ by decreasing density, and decreasing temperature will decrease $k^{\prime}$ by increasing the density and solvent power.

The mobile phase density is of primary importance in constant temperature work. When Giddings found that minimum pressures were needed to dissolve and migrate various samples ${ }^{49}$ he described a solubility parameter, $\delta$, to evaluate fluids in terms of density and compare supercritical fluids to other liquids. 50

$$
\delta=1.25 \mathrm{Pc}^{1 / 2}\left(p / p_{1 i q}\right)
$$

In this equation $\rho$ and $\rho_{1 i q}$ are the densities of the supercritical mobile phase and its density in the liquid state, and PC is the critical pressure of the mobile phase. As the solubility parameter of the mobile phase approaches $\delta$ of the solute, the solute dissolves and travels through the column. The effect of programming pressure or density can then be explained in terms of increased $\delta$ by increasing $p$. It has been found that at higher pressures the selectivity, $\alpha$, for homologous series is less. 28,51 This can be seen in figure 3 where a 
linear pressure program shows a noticeable difference between the degree of separation of early and late eluting components. Peaden and Lee have represented this effect by the following derivation. 40

For a homologous series at constant pressure the $\log k$ ' has been shown to be a constant a, related to the class of compound and further members of the series, each differing by a repeating unit " $n$ " increase $\log k^{\prime}$ according to

$$
\log k^{\prime}=A+B n
$$

where $B$ is the observed change in $\log k^{\prime}$ per repeat unit. ${ }^{28}$ The following relationship is also generally followed. 46,14

$$
\log k^{\prime}=a-b p
$$

where the constant a can be viewed as the $\log k^{\prime}$ of any solute under GC conditions at the same temperature and $b$ is the rate of change of log $k$ ' as a function of density.

Setting these equations to be equal and solving for the slope $B$.

$$
B=\frac{a-b p-A}{n}
$$

simplifying the constants gives

$$
B=B_{0}-\mathfrak{m p}
$$

where $B_{\circ}$ and $m$ are new constants. When this expression is substituted into Eqn 10, the following result is obtained. 


$$
\log k^{\prime}=A+B_{0} n-m n p
$$

Since selectivity (a) is defined as

$$
\begin{gathered}
\alpha=\frac{k^{\prime}{ }_{2}}{k^{\prime}{ }_{1}} \\
\log \alpha=\text { Bo }-\mathrm{mp}
\end{gathered}
$$

for two members of the same series. This result is the same as the value for the slope $B$ in Equation 13 obtained earlier.

Figure 5 shows data for alkanes and alkyl phthalates in supercritical pentane. 40 Extrapolation to $\log a=0(a=1)$ shows the point at which the homologs coelute. Although coelution does occur, it should be noted that the coelution points for the different chemical classes occur at different points which makes possible group type separations.

\section{Effects of Mixed Mobile Phases and Modifiers}

The use of additives in SFC has been found useful for many years. Methanol and isopropanol were used in several studies to decrease adsorption effects. Isopropanol and cyclohexane modifiers in pentane were used to elute polystyrene and both were found to decrease $k$ ' values. 48 This led to speculation that the modifier has two mechanisms of operation. The first is a simple adsorption model, where a polar modifier covers up active sites on the substrate surface, thus preventing sample adsorption and retention on that site and decreasing the time spent in the column. This is not the case, however, with cyclohexane which is non-polar but still decreased $k$ ' values. 


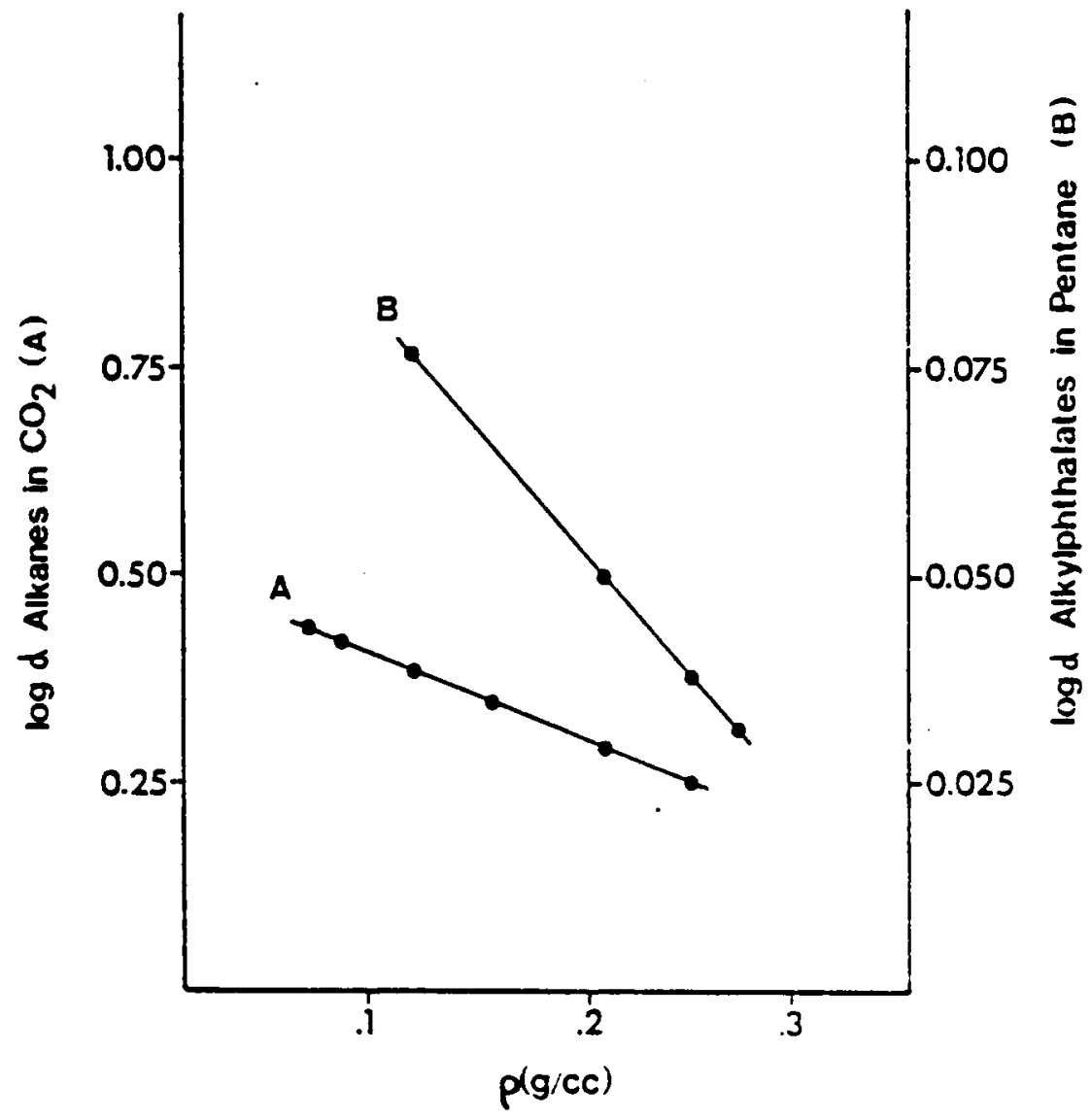

Figure 5. Selectivity as a function of density for A) alkanes in supercritical $\mathrm{CO}_{2}$ and $B$ ) alkylphthalates in supercritical pentane. 
Cyclohexane is a better solvent for polystyrene than is pentane, so this effect was proposed to be due to the presence of cyclohexane increasing the solubility of the polystyrene in the mobile phase.

On bonded phase packed columns using $\mathrm{CO}_{2}$, hexane was found to have no effect on $k^{\prime}$ at 0.5 weight while 0.5 weight methanol resulted in a 40 fold reduction in $k^{\prime} .52$ A recent investigation of the role of polar modifiers was made using fused silica capillary columns, which have an extremely inert surface compared to packed columns. ${ }^{53}$ at a low pressure $(78 \mathrm{~atm})$ and $100^{\circ} \mathrm{C}, 0.77$ mole methanol was found to have no effect on ' $k^{\prime}$ values compared to pure $\mathrm{CO}_{2}$. However, at higher pressures $168 \mathrm{~atm}$ and $100^{\circ} \mathrm{C}$ the same concentration of methanol was found to decrease the $k^{\prime}$ values of both polar and nonpolar molecules 8-138. Water was found to have no observable effect at $60^{\circ} \mathrm{C}$ and $84 \mathrm{~atm}(0.35$ mole 8 ), or at $168 \mathrm{~atm}$ and $100^{\circ} \mathrm{C}$ with 0.55 or 0.90 mole water. These data support the conclusion that the primary mechanism of $k$ ' reduction with modifiers on packed columns is surface adsorption of the modifier. On fused silica capillaries, the dramatic effects seen on packed columns are not observed, but some decrease is found at higher pressures due to an increase in the solvent power of the modified mobile phase. However, the relatively small $k^{\prime}$ changes associated with modifiers on fused silica do not indicate that the usefulness of this technique will be widespread for capillary applications. This is in strong contrast to the use of modifiers with packed columns where their use yields dramatic results and is so widespread that their use has become commonplace for most applications. The ineffectiveness of modifiers on fused silica is 
not a cause for concern or a drawback. It is instead a result of the lack of an adsorptive surface which permits the elution of compounds from capillary fused silica columns with pure $\mathrm{CO}_{2}$. These compounds require in many cases the addition of modifiers to allow their elution from of packed columns.

Structure/Solubility Relationships in $\mathrm{CO}_{2}$

Although supercritical $\mathrm{CO}_{2}$ and liquid $\mathrm{CO}_{2}$ have been utilized for many years as extraction solvents, correlation of the solubility of compounds has been difficult due to the wide variation of operating conditions. Two studies have attempted to remedy this situation the first by Francis in $1954^{54}$ and the second by Dandge et al. 55 in 1985 . The conclusions of the second, more comprehensive study are presented here. This study was conducted at $2500 \mathrm{psi}$ and either $25^{\circ}$ or $32^{\circ} \mathrm{C}$. No major changes in solubility were observed as a result of the $7^{\circ} \mathrm{C}$ temperature variation. The use of standard reference operating conditions permits relationships to be drawn between the structure of a compound and its solubility.

As a starting point, it was determined that under the set operating parameters, normal alkanes were completely miscible with $\mathrm{CO}_{2}$ up to carbon number 12. Above c-12 the solubility decreased rapidly with C-14 being only 168 soluble; $c-16$ is 88 soluble; and $c-18$ being only 38 soluble. Unsaturation has a favorable effect on the solubility of a compound. Compared to the 38 solubility of octadecane, 1-octadecene is 108 soluble. Branching of the molecule greatly increases its solubility with complete miscibility occurring in a range between $c-19$ and $c-30$ 
alkanes depending on the degree of branching.

Dicyclic compounds are found to be sparingly soluble in $\mathrm{CO}_{2}$. The only completely miscible dicyclic of those studied was indene. Solubility increased with increasing hydrogenation.

Naphthalene at 28 solubility is less than that of tetralin (148) which is less than decalin (228). Branching on the side chains of monoaromatics follows the same rules as for the aliphatics. The comparison is made between p-di-tert-butylbenzene and 1,3,5-tri-tertbutylbenzene which are completely miscible and 1-phenyl decane which is only slightly soluble.

In the case of alcohols, the solubility decreases rapidly after 1hexanol which is completely miscible. 1-heptanol is only 68 soluble and 1-decanol is only about 18 soluble. Once again, alkyl branching increases the solubility and phenyl substitutions slightly decrease solubility. Secondary and tertiary alcohols were found to be more soluble than primary alcohols while etherification greatly increased solubility due to decreased polarity and loss of intermolecular attractions.

Aliphatic carboxylic acids are completely miscible up to c-9 with solubility quickly dropping off so that by $c-12$ only 18 solubility is obtained. Esterification greatly increases solubility while halogen, hydroxy and aromatic substituents decrease solubility.

When amines are dissolved in dense $\mathrm{CO}_{2}$ care must be taken to prevent salt precipitation occurring as a result of the interaction of a basic amine and the slightly acidic $\mathrm{CO}_{2} \cdot$ Carbon dioxide has been 
observed to form salts with $\mathrm{NH}_{3}$ and aliphatic amines. Pyridine, however, is completely miscible and aniline has been determined to be 38 soluble. Basicity of the amine was found to exert a major effect on solubility. An upper limit of $\mathrm{K}_{b}=10^{-9}$ was suggested for samples to prevent salt formation. In aromatic amines solubility increases in the order of primary > secondary > tertiary.

Although these conclusions are valid only for the operating conditions studied, the comparison of structural relationships and substituents gives insight into the suitability of $\mathrm{CO}_{2}$ for various applications. It should also be noted that these are relatively mild conditions of temperature and pressure which could be increased to extend the solubility range of the $\mathrm{CO}_{2}$. In fact, it is the ability of $\mathrm{CO}_{2}$ to interact with samples over a wide range of temperatures and pressures that makes SFC an attractive technique. 
CHAPTER II

HISTORICAL

The Use of Supercritical Fluids for Extraction

In 1879 Hannay and Hogarth ${ }^{56}$ reported that compressed gases such as ethanol or tetrachloromethane when used above their critical points were able to dissolve metal halides due to their increased density. Following this initial discovery little use was made of this information. Industrially, the solubility properties of supercritical fluids proved to be a detriment when high pressure steam turbines precipitated dissolved salts on the turbine blades as the steam underwent decompression. In the petroleum industry solubility effects of supercritical fluids were seen in the processing of highly pressurized natural gas which entrained other compounds. 57,58

The first use of the solubility effects of dense gases was probably in 1943 by Messmore 59 at Philips Petroleum who proposed using supercritical hydrocarbons at high pressures to deasphalt oil. A process using supercritical propane was described in 1955 by zhuze and Kapelyushnikov ${ }^{60}$ to remove resin from crude oil. In 1958 a process for the extraction of lanolin from wool grease was described. 61

A few of the more recent applications of supercritical extraction technology include the use of supercritical toluene to extract coal in order to obtain a more concentrated hydrogenated fraction ${ }^{62}$; the decaffeination of green coffee with $\mathrm{CO}_{2}{ }^{63}$; and the use of counter current processes with supercritical $\mathrm{CO}_{2}$ to separate monoglycerides from diglycerides on a commercial scale. 64 The use of supercritical fluids 
in the extraction of hops, spices and tobacco is reviewed by Hubert and vitzthum. 15

\section{Early SEC}

The merging of supercritical fluid extraction technology and the newly developed technique of SFC took place in the late $1950^{\prime} s$. It has been reported ${ }^{65}$ that Lovelock was the first to propose the use of a supercritical mobile phase at an international GC meeting in 1958, although the first experimental results were not published until 1962 by Klesper et al. 1

This initial application of SFC was the separation of nickel porphyrins with dichlorodifluoromethane and monochlorodifluoromethane. The initial work was referred to as "dense gas chromatography" (DGC). The work of Klesper was expanded by sie, Rijnders et al. $(28,33,47,66,67)$ who introduced the use of such mobile phases as carbon dioxide, isopropanol, and $n$-pentane to a wide variety of samples including PAH's and dyes. Sie and Rijnders divided SFC into two parts, FSC (fluid solid chromatography) and FLC (fluid liquid chromatography) depending on whether the column packing consisted of a solid adsorbant or a liquid coated particle. Many of the initial observations on the effects of pressure and column particle size date from this work.

High pressure gas chromatography was the description given to SFC by Giddings et al. in the late 1960's to work performed with $\mathrm{CO}_{2}$ or $\mathrm{NH}_{3}$ as the mobile phase. 26,49 A varied selection of applications were developed including polymers, amino acids, and sugars. This work on 
"migration" of solutes appeared very promising at the time, but was soon overshadowed by the rapid advances in capillary GC and HPLC.

In the early 1970's Jentoft and Gouw ${ }^{68}$ demonstrated the application of SFC to $C_{8}-C_{20}$ alkyl bromides and separated various metal chelates, ferrocene, cyclopentadienyl $\mathrm{Mn}(\mathrm{CO})_{3}$ and dicyclopentadienyl $\mathrm{TiCl}_{2} \cdot$ Some of their improvements to the technique included a modified mercury displacement pump for pressure control up to $5000 \mathrm{psi}$ and the use of pellicular as opposed to porous packing materials. They were also able to trap and analyze PNA's from automobile exhaust. 69 one of their major contributions was certainly the publication of an excellent review of SFC theory and applications which summarized much of the theoretical and instrumental work of the preceding ten years. 70

Bartmann and schneider investigated the effects of pressure programming in addition to studying the effects of flow rate on efficiency and the fit of these data to a generalized van Deemter equation. 30,36 Also included in this study was an analysis of solubility effects during the injection process.

Rlesper described an instrument which allowed independent control of both pressure and temperature during the chromatographic run and showed the separation of styrene oligomers of $M_{w}=2200$ on a column packed with Porasil using pentane and pentane/methanol mixtures as supercritical fluids. 57

The first SFC/MS combination was described in 1978 by Randall and wahrhafting. ${ }^{71}$ A molecular beam interface into a mass spectrometer allowed mass spectra of several compounds to be obtained including 
anthracene, triphenyl benzene, phenanthrene and azobenzene.

Condensation cluster formation was observed with several gases including ethane and $\mathrm{CO}_{2}$. Problems were reported with plugging of the interface orifice which was partially solved by repeated filtering of the samples. Two contrasting effects of modifiers were described by Conaway et al. for the elution of polystyrene oligomers. ${ }^{48}$ while additions of both isopropanol and cyclohexane to pentane at less than $58 \mathrm{v} / \mathrm{v}$ caused more complete elution of the sample, increased percentages of cyclohexane decreased $k^{\prime}$ values further while increases of isopropanol above 58 had no additional effect. This observation was explained by suggesting that isopropanol addition deactivated adsorption sites on the column while cyclohexane enhanced the solvent power of the supercritical fluid pentane. Non-linear pressure programming was shown to elute homologous series with equal spacing between peaks, while inverse temperature programing had erratic effects on the separation near the critical point. Graham and Rogers reported that for packed columns with particle sizes in the region of 100 to $200 \mu \mathrm{m}$ resolution was increased when a fast programming rate was used with a high flow rate. Operation at linear velocities which produced turbulent flow in the columns was found necessary for maximum resolution. 43

For the analysis of oligomeric styrenes, Schmitz and Rlesper modified a commercial HPLC instrument (Hewlett-Packard 1084B) so that in addition to temperature and pressure gradients, mobile phase composition gradients could also be generated. The mobile phase used was pentane and the composition was changed by the addition of increasing amounts of either cyclohexane or 1,4 dioxane during the chromatographic analysis. 
Using eluent gradients polystyrenes with $M n=3470$ were successfully analyzed. 72,73

\section{Modern Developments in SFC}

In 1981, a joint announcement was made by Novotny et al. at Indiana University and Lee et al. at Brigham Young University of the use of open tubular columns for supercritical fluid chromatography. ${ }^{2}$ This development was made possible by the development of both GC and BPLC technology. The original work was performed with glass capillary columns of 0.2 and $0.3 \mathrm{~mm}$ i.d. using $n$-pentane as a mobile phase and both ultraviolet and fluorescence detection.

The prospect of using $\mathrm{CO}_{2}$ as a mobile phase for capillary SFC was first proposed by springston and Novotny in a theoretical investigation dealing with optimal and practical parameters of the technique. ${ }^{74}$ They recommended the use of $120 \mu \mathrm{m}$ i.d. columns which should generate $10^{6}$ theoretical plates in 13 hours when operated at ten times the optimum linear velocity.

A description of a capillary SFC instrument and some selected applications were made by Peaden et al. ${ }^{21}$ Pyrex glass capillaries were dynamically coated with a methylphenylpolysiloxane stationary phase. Internal diameters of 0.11 and $0.13 \mathrm{~mm}$ were used to analyze coal tar and a carbon black extract. Analysis times were in excess of two hours. Spectrofluorometric detection was used with the end of the pyrex capillary column serving as a flow cell.

A direct fluid injection interface for capillary SFC/MS was described by smith et al. 75 This work used fused silica capillary 
columns of 0.1 and $0.2 \mathrm{~mm}$ i.d. with isobutane or n-pentane as the mobile phase. The design used fused silica transfer lines to either a laser drilled $0.5-2.0 \mu \mathrm{m}$ orifice or to a pinched platinum-iridium restrictor which provided a connection to a chemical ionization source. The application of the SFC/MS system to PAH's and to an oligomeric polystyrene sample was demonstrated.

SFC theory in 1982 was advanced by physical measurements of diffusion coefficients by Feist and schneider ${ }^{76}$ and by an investigation of linear density programming for extraction and packed column work by Bowman et al. 25 A development which had profound impact on the general acceptance of SFC as an analytical technique was the introduction of a modified commercial HPLC system by Hewlett-Packard in 1982. The availability of a commercial instrument not only made possible the opportunity for many laboratories to use SFC but also provided an instrument design on which applications could be standardized. The initial work by Gere and co-workers $46,52,77$ described necessary hardware modifications, effects of particle size and packed column internal diameter and the use of various mobile phase modifiers. Applications covering a wide range from caffeine in beverages and PAH's to bacterial cell extracts were presented. The effects of several different mobile phases were also characterized by Lauer et al. ${ }^{18}$ who demonstrated the use of $\mathrm{CO}_{2}, \mathrm{~N}_{2} \mathrm{O}$ and $\mathrm{NH}_{3}$ on bonded phase silica gel columns and polymeric packings such as the PRP-1 (Hamilton Co., Reno, Nevada).

* In the development of capillary SFC, Novotny and Springston ${ }^{78}$ studied the effect of column diameter and flow rate on H.E.T.P., using butane as the mobile phase. While proposing that packed microcolumns 
(0.2-0.8 $\mu \mathrm{m}$ i.d. with $3 \mu \mathrm{m}$ particles) might be useful for SFC, it was also stated that progress in SFC would be greatly dependent on the development of immobilized, selective capillary stationary phases. Fjeldsted et al. ${ }^{20}$ demonstrated a density programmable SFC instrument utilizing an Apple Plus computer to generate various density ramps by controlling both the column temperature and pressure during the analysis. The effectiveness of this technique was demonstrated for polystyrene $M_{w}=2000$. A theoretical treatment of resolving power for SFC by Peaden and Lee ${ }^{31}$ discussed the importance of thick film capillaries for high sample loadings and the effects of pressure drops across the column on resolution. Grob reviewed the practicality of SFC and concluded that although an alkane mobile phase such as hexane was well suited for SFC since its relatively high critical temperature and low critical pressure allows the elution of compounds based on volatility and extractability combined, $\mathrm{CO}_{2}$ is preferred due to safety considerations. It was noted that the lower concentration limit for explosion of hexane in air $(0.88)$ makes this a dangerous mobile phase when combined with an operating temperature in excess of $240^{\circ} \mathrm{C} .79$ The development of detectors for SFC continued with a report of capillary SFC/FID and a capillary SFC/NPD by Fjeldsted et al. ${ }^{80}$ The interface was accomplished using a $9 \mathrm{~cm}$. $10 \mathrm{\mu m}$ i.d. restrictor inserted between the column and the detector. Noise spiking was reported with this interface which necessitated the installation of a low pass filter to electronically remove the spikes. Spiking was attributed to aersol particles entering the detector and causing ion bursts. A scanning 
fluorescence detector for capillary SFC was also reported by Fjeldsted et al. 81 This detector was capable of being operated in both a fixed wavelength and scanning mode, and used optical fibers to decrease stray light background and provide a light pipe from the cell to the entrance of the monochromator. The design of this detector had a low dead volume appropriate for capillary applications and quantities as low as $1 \mathrm{ng}$ were reported to yield full spectra. An interface for capillary SFC/FTIR was demonstrated for the first time by Shafer and Griffiths ${ }^{82}$ using a relatively large $60 \mathrm{~m} \times 0.33 \mathrm{~mm}$ open tubular column. It was reported that a relatively long $(1 \mathrm{~cm})$ pathlength could be used with $\mathrm{CO}_{2}$ compared to short $(0.2-0.5 \mathrm{~mm})$ pathlengths needed for HPLC/FTIR. A potential problem was reported concerning the increase in absorption bands of $\mathrm{CO}_{2}$ during pressure programming. Although the authors were able to spectrally substract these bands in the region of 1200-1400 $\mathrm{cm}^{-1}$, this resulted in a reduction in the signal to noise ratio. Gradient elution for packed column SFC was further investigated for the separation of polystyrenes by schmitz et al. ${ }^{83}$ Diethyl ether as a mobile phase with ethanol or dioxane was investigated and compared to pentane as a mobile phase with cyclohexane or dioxane as modifiers. The best results were obtained with pentane/dioxane as a mobile phase. Problems were reported due to silica gel dissolution when alcohol was present in the gradient at concentrations greater than 5-108 $\mathrm{v} / \mathrm{v}$. An investigation of the solvent power of $\mathrm{CO}_{2}$ and the effect of chemical composition and concentration of various modifiers was made by Randall 17 using solvent selectivity triangles as proposed by snyder. 84,85 
Capacity ratios of phenyl esters of fatty acids in $\mathrm{CO}_{2}$ were determined by wilsch et al. Better separation was reported when octyl bonded porous layer beads were used instead of bare silica beads. Pjeldsted and Lee ${ }^{88}$ described some advances in instrumentation for capillary SFC in 1984. These advances included the use of microprocessors to generate asymptotic density gradients. This was reported to increase resolution of later eluting peaks by decreasing the rate of density increase near the end of the chromatographic run. An increase in sensitivity in UV absorption and fluoresence detectors was reportedly gained by cooling the supercritical fluid to a liquid before detection. This effect was attributed to better beam collimation due to the higher refractive index of the liquid state compared to the supercritical state and also to band compression occurring during the cooling process which yielded higher solute concentrations. The use of the liquid phase in the detector cell also eliminated the need to insulate and regulate the temperature of the detector cell, since in many cases ambient temperatures could be used. A comparison of GC and SFC for a coal tar sample was presented and it was shown that the resolution possible for SFC approached that of GC although a 120 minute analysis time was needed to approach the resolution of a 60 minute capillary GC analysis time.

A U.S. Patent was awarded in 1984 to Novotny, Lee et al. which covered practically all aspects of capillary SFC including pumps, injection systems, capillary columns, the use of polymethylpheny1siloxanes stationary phases, various detectors, and data collection systems. 89 This patent was assigned to Brigham Young University. 
The deactivation of small ( $50 \mu \mathrm{m}$ i.d.) diameter columns for SFC was reported by Kong et al. 90 Deactivation was accomplished by the use of either thermally degraded ov-101, octamethylcyclotetrasiloxane $\left(D_{4}\right)$, hexamethyldisiloxane or hexamethyldisilazane (HMDS). The ov-101 gave poorer results than the other three methods. There was found to be no significant difference between $D_{4}$, HMDS and the hexamethyldisiloxane deactivation, although the use of $\mathrm{D}_{4}$ was reported to increase the possibility of column plugging during deactivation. All of these techniques were suitable only for non-polar columns since the deactivation treatment produces a low energy methylated surface.

The coating of small diameter capillary columns for SFC was reported by kong et al. 91 phases coated included SE-33, SE-54, and a 508 phenyl polysiloxane. Azo-tert-butane was used as a free radical crosslinking agent and films up to $1 \mathrm{~mm}$ in thickness were reported. The use of elevated coating temperatures and mixed solvents for the stationary phases made possible the coating of these columns. The effect of column diameter on efficiency was studied by Fields et al. 92 while noting that smaller diameters ( $25 \mu \mathrm{m}$ i.d.) would give the greatest resolution per unit time, it was stated that $50 \mu \mathrm{m} i . d$. columns would be better in terms of overall efficiency when time was not a factor. Since SFC columns have a more favorable ratio of mobile phase diffusion coefficients to stationary phase diffusion coefficients compared to GC, thicker films could be utilized in SFC without experiencing a severe loss in efficiency. Film thicknesses of up to $1 \mu \mathrm{m}$ were found to be useful without major efficiency losses. The increased sample capacity 
of thick film columns was found to decrease the demands on detector sensitivity.

Springston and Novotny investigated the solute diffusivities of several PAH's in supercritical alkanes using uncoated capillary columns. 94 Klesper et al. $95-97$ reported that sub and supercritical dimethyl ether gave better chromatographic resolution on packed columns than diethyl ether. In addition it was found that increasing the temperature of the mobile phase caused a decrease in resolution below the critical temperature and further temperature increases improved resolution as the mobile phase passed its critical point. The increase in resolution passes through a maxima and then decreases with increasing temperature. This was reported to be due to a decrease in the density which increases resolution until increased vaporization of the sample at higher temperatures caused increased band broadening. This led to a decrease in $k^{\prime}$ and resolution. A review of phase equilibria and diffusion properties of supercritical fluids as applied to chromatography was presented by schneider. 98 schoenmakers presented a thermodynamic model for SFC that was in good agreement with experimental data. 38

Packed column applications were extended at this time by several laboratories. Norris and Rawdon ${ }^{99}$ demonstrated group type separation of gasoline and kerosene on a silver nitrate impregnated silica gel column and showed a favorable comparison between this chromatographic method and the fluorescent indicator adsorption method (FIA) ASTM D 1319. A further application of SFC to metal chelate analysis was reported by 
46

Wenclawiak and Bickmann ${ }^{100}$ for the diethyldithiocarbamidates of $\mathrm{Cu}, \mathrm{Co}$, $\mathrm{N} i$ and $\mathrm{zn}$, the oxinates of $\mathrm{Pt}, \mathrm{Pd}, \mathrm{Ir}, \mathrm{Ru}$, and $\mathrm{Rh}$, and the acetylacetonates of $\mathrm{V}, \mathrm{Cr}$ and Fe. Hirata ${ }^{101}$ showed the separation of ov-17 phenylmethylpolysiloxane and dialkylphthalates using pentane and ethanol modified pentane. Wright et al. expanded the applications of capillary SFC to marine diesel fuel 102 and middle distillate fuels. 103 The use of chemical ionization (CI) mass spectrometry with isobutane as a reagent gas and $\mathrm{CO}_{2}$ as a mobile phase gave excellent results for the marine diesel fuel. Carbon dioxide was found to be an excellent mobile phase for the middle distillate fuel and was found to elute even nitrogen-containing and hydroxylated PAH's. Rawdon and Norris ${ }^{104}$ published an interesting study comparing packed SFC with capillary SFC run well above its optimum linear velocity on the same sample. Although the capillary was admittedly not optimized, similar analysis times and increased resolution on the capillary suggested that fast analysis on GC capillary columns might be feasible. Rawdon also introduced a flame ionization detector for packed SFC columns by flattening a F.I.D. flame jet with pliers. This was reported to give acceptable results with $\mathrm{CO}_{2}$ as a mobile phase.

Chester $^{105}$ reported a fused silica restrictor for F.I.D.'s drawn to a taper in a flame. This tapered restrictor was found to significantly decrease detector spiking in flame ionization detectors. Smith et al. 106 showed a comparison of 2 meter and 15 meter $50 \mu \mathrm{m}$ i.d. columns used with mass spectrometric detection. Detection limits were found to 
be about $0.1 \mathrm{pg}$ for biphenyl compounds using single ion monitoring. The same group also reported on the ability to obtain electron impact ionization mass spectra by utilizing the lower gas volumes generated by 50 micron internal diameter columns. 107

$\downarrow$ Two different interfaces for SFC/FTIR were developed in 1984. The first interface for microbore SFC was a diffuse reflectance infrared Fourier transform (DRIFT) interface which deposited the sample on powdered $\mathrm{KCl}$ and the solvent was removed by evaporation and the components analyzed. ${ }^{108}$ The gaseous state of $\mathrm{CO}_{2}$ at S.T.P. simplified the interface by allowing easy removal of the mobile phase. This interface permits the use of modifiers since a mobile phase containing 58 methanol gave no methanol spectra at the detector, indicating that the solvent mixture had been completely removed. Detection limits of less than $10 \mathrm{ng}$ were predicted for this design. The second approach involved the use of a high pressure flow cell for capillary SFC. Olesik et al. 24 evaluated carbon dioxide, propane and dichlorodifluormethane as solvents for SFC/FTIR but found that only $\mathrm{CO}_{2}$ had the necessary degree of transparency at high pressures. Pressure programming during the chromatographic run was performed but this led to a steadily increasing background for the Gram-Schmidt reconstruction.

A new development in column technology was the development of fused silica packed capillary columns. ${ }^{109-112}$ These columns were used with both carbon dioxide and ethanol modified hexane as mobile phases. The results shown with these packed microcolumns are very similar to those obtained with packed HPLC type columns. The principal advantages for 
this technique seem to be the low solvent consumption and the more rapid thermal equilibration of the capillary packed column compared to a larger packed column.

In 1985 Richter suggested a modification of the SFC-FID which incorporated a secondary heating block before the detector which maintained the restrictor connector at a high temperature $\left(400^{\circ} \mathrm{C}\right) .113$ This design was found to decrease spiking at the detector at higher pressures for some compounds. For example, the results with erythromycin A showed some degradation products which were not found with the original FID design presumably due to adsorption on cold spots in the detector.

( More applications of the SFC technique were introduced in 1985. Wright and Smith showed the separation of four carbamate pesticides in 90 seconds using a very short $(0.9 \mathrm{~m})$ column with a small i.d. (25 $\mu \mathrm{m}) .114$ The analysis of these thermally labile pesticides was of interest since operating temperatures above $75^{\circ} \mathrm{C}$ were found to cause sample decomposition. The application of SFC/MS to tricothecene mycotoxins was shown by Smith et al. ${ }^{115}$ SFC was found to be appropriate for the sensitive detection of these labile compounds. One aspect of particular interest was the observation that well deactivated columns were necessary for the elution of polar samples. An example of the need for selective stationary phases for capillary applications was given by White and Houck for the analysis of mono-, di-, and triglycerides. 116 They found that a polar (DB-225) column permitted the elution of triglycerides in order of increasing unsaturation. This increased 
retention of unsaturated compounds is due to weak pi complexes being formed between the nitrile groups of the stationary phase and the double bonds of the analyte.

Kuei et al. demonstrated that an n-octyl methyl polysiloxane stationary phase was useful for SFC. ${ }^{117}$ The octyl alkyl groups emanating from the siloxane backbone were found to have an increased interaction with sample molecules and therefore greater retention. The phase was also reported to be easily crosslinked due to the abundance of readily extractable methyl hydrogens.

$X$ Further advances in SFC-IR were reported in 1985. Fujimoto et al. reported on the use of an IR detector. This work used capillary packed columns with ethanol modified hexane as a mobile phase. The sample was deposited on a continuously moving $\mathrm{KBr}$ plate and the solvent was removed by evaporation. The deposited sample was then placed in an IR spectrophotometer and absorption was monitored. The use of an IR rather than an FTIR resulted in longer scan times but this was not a problem due to the off-line nature of this technique. Johnson et al. showed a high pressure light pipe flow cell for on-line packed column SFCFTIR. 119 The $4 \mu \mathrm{L}$ cell made of znse permitted detection limits of about 270 ngs for acetophenone. Pressure changes in the $\mathrm{CO}_{2}$ spectra were found to be less than previously reported by Shafer and Griffiths. In 1986 further attempts were made to improve the detectors available for SFC. Guthrie and Schwartz described an "integral restrictor" for SFC. ${ }^{120}$ This restrictor was made by flame sealing the end of the capillary column and then carefully abrading the molten 
droplet formed until a small orifice is revealed. This small $(\sim 1-3 \mu \mathrm{m})$ opening provides a short restriction between the SFC mobile phase and the FID which is reported to exhibit no spiking. SFC-FTIR using an IR microscope was reported by Pentoney et al. 121,122 This hyphenated technique used a capillary column to separate the sample which was then deposited on a $\mathrm{znSe}$ moving window. The IR microscope was used to condense the infrared beam down to a suitable size for the sample spot $(\sim 200 \mu \mathrm{m}$ i.d.). Full spectra were obtained for as little as $30 \mathrm{ng}$ of component $\mathbb{N}$ a thermionic detector was described by west and Lee for capillary SFC. ${ }^{123}$ Several modes of thermionic detection were evaluated and detection limits were in the nanogram to subnanogram range for nitrogen containing compounds. $K$

Ion mobility detection for SFC was first published by Eatherton et al. in $1986 .^{124}$ This technique uses atmospheric pressure ionization in a constant electric field to first ionize the sample as it elutes from the column and then resolve the components based on the differences in time required to drift across the tube. The instrument can be operated as a general detector in a total ion mode or only specific drift time windows can be monitored in the selective mode. Compounds with molecular weights of over 800 Daltons were detected and high molecular weight did not seem to be a limiting factor for this separation technique.

A new development in SFC/MS described the use of a benchtop (HP 5970) mass selective detector with capillary SFC. ${ }^{125}$ This system was able to provide useable mass spectral information by using the MSD with 
its optional chemical ionization (CI) attachment. Excellent signal to noise ratios were obtained with amounts as low as $30 \mathrm{ng}$. Caffeine and fatty acid methyl esters were found to give strong $(M+1)^{+}$molecular ion peaks. The only drawback to this technique is the relatively low information. content of CI spectra compared to the fragmentation patterns obtained with electron ionization (EI).

Applications of SFC continued to increase in 1986. Later et al. used SFC to separate various drugs to determine the suitability of SFC as a drug analysis technique. ${ }^{126}$ The drugs used in this study included tetrahydrocannabinol and its metabolites, cocaine, phencyclidine, phenobarbital and emthandone. The low temperatures of the SFC analysis were found to be helpful in preventing decomposition and/or rearrangement of the compounds studied. Markides et al. showed the analysis of free carboxylic acids. ${ }^{127}$ Polymethylhydrosiloxane was used as a deactivation reagent and was reacted with the column surface at $300^{\circ} \mathrm{C}$ for 5 hours to provide a deactivation layer for a non polar column. A polar (508 cyanopropyl methyl polysiloxane) column was deactivated with 508 cyanopropyl methylhydropolysiloxane at $250^{\circ} \mathrm{C}$ for 10 hours. Separation of five unsaturated C-18 fatty acids was shown on the 508 cyanopropyl methylpolysiloxane column.

Jackson and Later showed capillary SFC separation of commercial azo, aniline and anthraquinone dyes. ${ }^{128}$ These polar dye samples were chromatographed with a UV-visible spectrophotometer using pentane as the mobile phase. Even though the column used was coated with a non-polar phase (SE-54), the dyes were found to separate based on chemical functionality rather than molecular weight. These preliminary results 
suggest that SFC may become an important tool in dye analysis.

Chester and Innis presented data on separations of polyglycerol esters containing 3-5 hydroxyl groups and the silyl derivatives of these esters. 129 Although the underivatized esters were able to be chromatographed, derivatization increased the resolution of the sample by sharpening the peaks and moving the elution of the peak to a lower pressure. No difference in selectivity was found when a DB-17 column was used instead of a $\mathrm{DB}-1$. Derivatization gave the most dramatic increase in resolution and reduced retention time with more highly hydroxylated samples.

The analysis of bacterial glycerol tetraether lipids by capillary SFC was reported by Deluca et al. ${ }^{130}$ These bacterial cell extracts were glycerol tetraethers joined by an isopranyl side group which differed only in the number of cylclopentane units incorporated in the linkage. A difference of one cyclopentane unit was found to give good separation. Six lipids were separated in 20 minutes on a 508 phenyl methylpolysiloxane coated column.

Proot, Sandra and Geeraert showed the results of a study on the separation of triglycerides. ${ }^{131}$ Four triglyceride standards were evaluated at different temperatures. The best temperature for this separation was found to be $150^{\circ} \mathrm{C}$. At this temperature the results of a quantitative analysis gave a relative standard deviation of the percentage of each compound in the mixture less than one per cent. The relative standard deviation of the actual area counts for each peak was not given. The authors recommend analysis temperature of $150^{\circ}-230^{\circ} \mathrm{C}$ for 
SFC due to the higher mobile phase velocities and increased

diffusivities at these temperatures.

Schwartz et al. have shown that capillary SFC can be used to extend the range of samples amenable to simulated distillation. ${ }^{132}$ Packed column GC was found to elute components up to $1000^{\circ} \mathrm{F}$ in boiling point. The use of short capillary GC columns has been reported to analyze fractions up to $1400^{\circ} \mathrm{F}$, but high column temperatures (up to $430^{\circ} \mathrm{C}$ ) were necessary. Capillary SFC was able to analyze the same sample at only $100^{\circ} \mathrm{C}$. This was considered important since fossil fuels can decompose at temperatures as low as $350^{\circ} \mathrm{C}$. Further extension of the boiling point range can be made by lowering the temperature and increasing the density.

At the 1986 Pittsburgh Conference, much interest in SFC was expressed. A variety of papers dealt with various aspects of the technique. Markides et al. ${ }^{133}$ reported on the use of hydropolysiloxanes for column deactivation and the preparation of thick film (up to 2 micron) columns for SFC. Guthrie ${ }^{134}$ presented applications using the integral restrictor and a bonded polyethylene glycol stationary phase. Kumar et al. 135 showed applications ranging from triglycerides to fluorocarbons. Calvey et al. ${ }^{136}$ showed packed column SFC separation of Alternavia toxins in food products and compared the SFC method to the HPLC method for a tomato product. 'Jordan et al. ${ }^{137}$ showed the use of packed column SFC-FTIR for fatty acids in several complex mixtures including butter and soap. Ashraf-Khorassani et al. ${ }^{138}$ showed the separation of metal acetoacetonates on a phenyl packed column. Owens and Reilly ${ }^{139}$ presented the results of a study using laser induced 
54

fluoresence/ionization for selective monitoring of SFC effluents. Sin et al. 140 showed the application of SFC/Supersonic Beam Mass Spectrometry to carbazole and phenanthrene. A Nd:Yag laser was scanned through the UV region and multiphoton ionization spectra obtained. Jesforques and Perrot ${ }^{141}$ showed the application of preparative scale SFC using up to a $12 \mathrm{~cm}$ i.d. column to a tetraline sample. This work suggests a possible application to pharmaceutical and related. industries. Richter ${ }^{142}$ introduced a new multipath restrictor for use in SFC/FID. Chester ${ }^{143}$ presented applications of SFC-FID for fats, carbohydrates and surfactants. Wright ${ }^{144}$ et al. presented applications of capillary SFC using $\mathrm{CO}_{2}$ with methanol and acetonitrile modifiers. 
CHAPTER III

INSTRUMENTATION FOR CAPILLARY SFC

\section{Introduction}

The instrumental aspects of capillary SFC require more rigorous equipment and material design than any other chromatographic technique. Capillary SFC relies heavily on the technology which has been developed in the last few years for GC and HPLC. The equipment borrowed from these techniques must be carefully matched with the operating temperatures and pressures utilized in SFC. In many cases, instrumentation is a compromise between the needs of the chromatographic separation and the needs of the detection system utilized. In addition, economics and analysis time must be considered in the design of practical instrumentation. The instrument that is described here is a result of the application of chromatographic principles and experience to the problem of designing a versatile capillary SFC system. The success of the system is dependent on each of its various parts. Each aspect of the system must be considered to achieve optimal performance. The evolution of the parts of the capillary SFC system used in this work will be discussed in turn. Commercial instrumentation for capillary SFC was unavailable until March 1986, so the instrumentation used in this work was assembled from many sources. A schematic of the instrumentation developed is shown in Figure 6 .

\section{Mobile Phase}

The choice of mobile phase was dictated early in this work. Only $\mathrm{CO}_{2}$ had the necessary combination of characteristics for general use. 


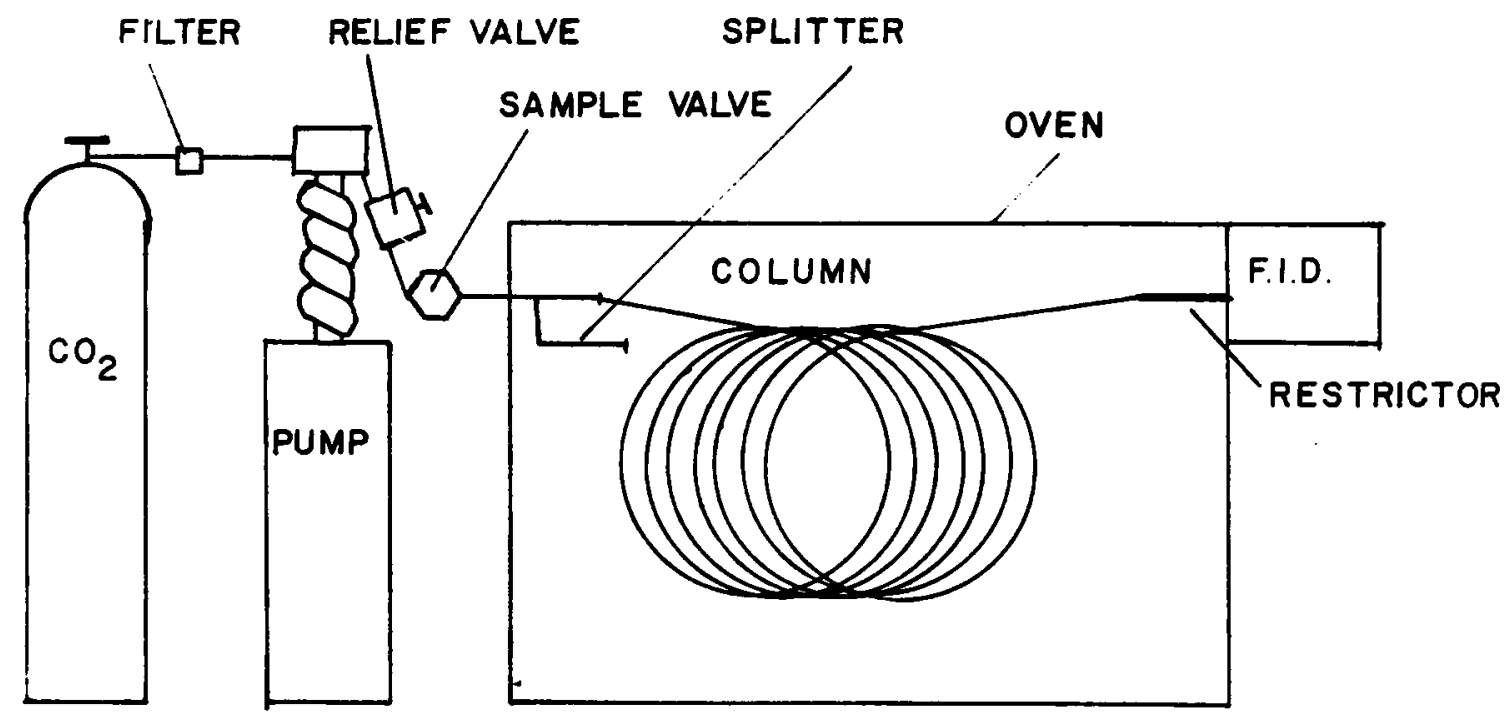

Figure 6. Schematic of a SFC equipped with a flame ionization detector. 
The reasonable critical temperature and pressure of $\mathrm{CO}_{2}$ along with its suitability for flame ionization detectors and its limited toxicity combined to make it the mobile phase of choice. $\mathrm{CO}_{2}$ was obtained in a steel cylinder from Airco (Montvale, NJ) in a purified grade. Carbon dioxide is normally obtained as a liquid under its own vapor pressure ( -800 p.s.i.). The $\mathrm{CO}_{2}$ may be dispensed as either a gas (from the top of the cylinder) or as a liquid from a dip tube inserted below the liquid level of the tank. In general, it was found to be more convenient to use a dip tube to transfer liquid $\mathrm{CO}_{2}$. The purity of the $\mathrm{CO}_{2}$ is of paramount importance. With FID detection, trace levels of organic materials in the part per million range are easily detected. $\mathrm{CO}_{2}$ from our initial source was found to be quite dirty, as indicated by a steadily rising baseline terminating in a "hump" during pressure programming. Subsequent investigation involving the elimination of other possibilities for this effect indicated that the $\mathrm{CO}_{2}$ contained organic material which concentrated on the column at low densities and then eluted at high densities. Although precise identification was impossible, the material was presumably an oil which contaminated the cylinder during the refilling process. When $\mathrm{CO}_{2}$ (Coleman Instrument Grade) was obtained through Matheson (Dorsey, MD), this problem was greatly diminished and with the subsequent availability and use of a special SFC grade from Scott Specialty Gases (Plumsteadville, PA), this problem disappeared.

Using capillary columns permits great solvent savings. A single tank of $\mathrm{CO}_{2}$ easily lasts for more than six months. This low solvent 
consumption makes even expensive gases affordable. Therefore, capillaries could be used with gases that are hard to purify in large quantities or with gases like Xenon which are simply very expensive. The transfer of $\mathrm{CO}_{2}$ to the pump was accomplished with stainless steel tubing which was first methanol rinsed and then blown dry with compressed gas before use. In addition, an in-line, $2 \mu \mathrm{m}$ filter was installed between the tank and the pump to prevent particulates from entering and damaging the pump seals. The use of activated silica gel columns to remove organics was tried for several days but had no observable effect and was removed. With highly impure gas, it is unlikely that the trap would be effective in adsorbing much material from liquid $\mathrm{CO}_{2}$, particularly the methane gas which is a primary contaminant. With the highly purified gas currently in use, a chemical trap is unnecessary. In addition, a trap provides a pressure drop between cylinder and tank and slows liquid transfer during a refill.

\section{Punp}

The pump must meet stringent criteria to operate effectively. The most important requirement is the ability to deliver pulseless flow at low flow rates and high pressures. In addition, the pump should be able to maintain a constant pressure and be capable of generating pressure ramps up to 5000 psi. A pump with these features was not available until last year so one had to be built using a commercially available syringe pump.

Pressure control by the pump is necessary for use with open tubular columns. The primary reason is that pressure control provides the most 
accurate and reproducible regulation of linear velocity at low flow rates. In addition, the open tubular column would be subjected to a large change in flow rate caused by pressure pulses during injection if flow control were used. This sensitivity to pressure pulses prohibits the use of reciprocating piston pumps. A pump with pressure pulsations would transport the sample through the column in a jerky manner which would cause chromatographic band broadening and subsequent loss of efficiency.

The syringe pump provides pulseless flow at low flow rates and also provides a high pressure source for the analysis (Figure 7). The movement of the piston is controlled by the motor speed and the gear assembly. After surveying the commercially available instrumentation, an ISCO $\mu \mathrm{LC} 500$ Micropump (ISCO, Inc., Lincoln, NE) was chosen for the system. This pump was designed for use with microbore (1 $\mathrm{mm}$ i.d.) HPLC columns. A $50 \mathrm{~mL}$ solvent reservoir was found to be sufficient for about 5-6 hours of continuous use before refilling. This pump was able to deliver solvent flow rates in the range of $0.2 \mu \mathrm{L} / \mathrm{min}$ to $600 \mu \mathrm{L} / \mathrm{min}$. which were appropriate for SFC applications.

A maximum pressure limit of 10,000 p.s.i. exceeded the maximum pressure limits of other system components, therefore, the pump was not the limiting factor in high pressure use. The pump was equipped with Teflon seals which resist swelling and dissolution by 1 iquid $\mathrm{CO}_{2}$. In addition, the internal solvent reservoir was easily cooled by the attachment of a coiled 1/8" copper tubing jacket connected to a Neslab (Model RTE-5B) (Portsmouth, NH) recirculating cooling bath (Figure 8). The cooling of the pump permitted $\mathrm{CO}_{2}$ to be transferred as a liquid from 


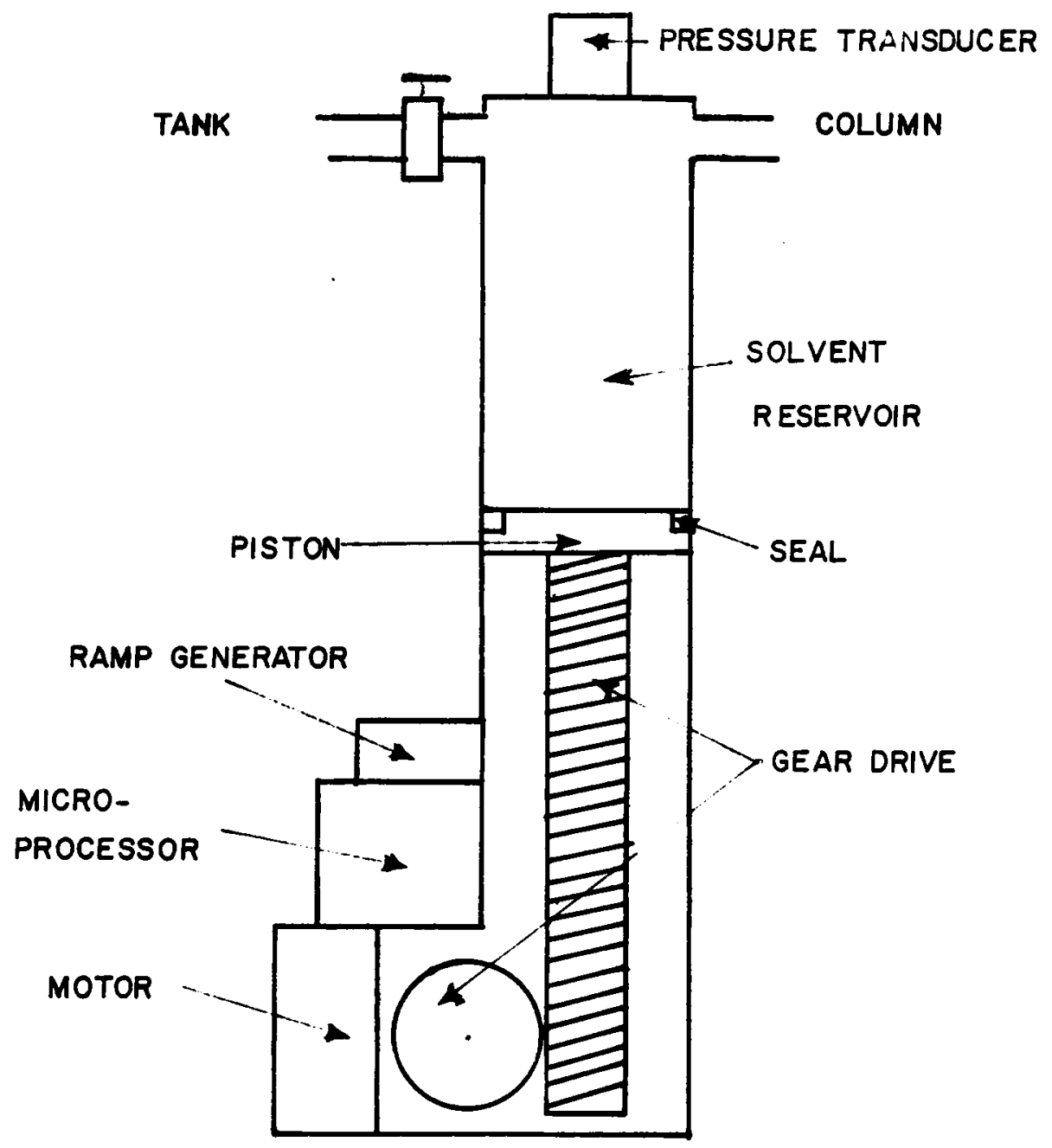

Figure 7. Schematic of a SFC syringe pump modified for pressure control and programming. 


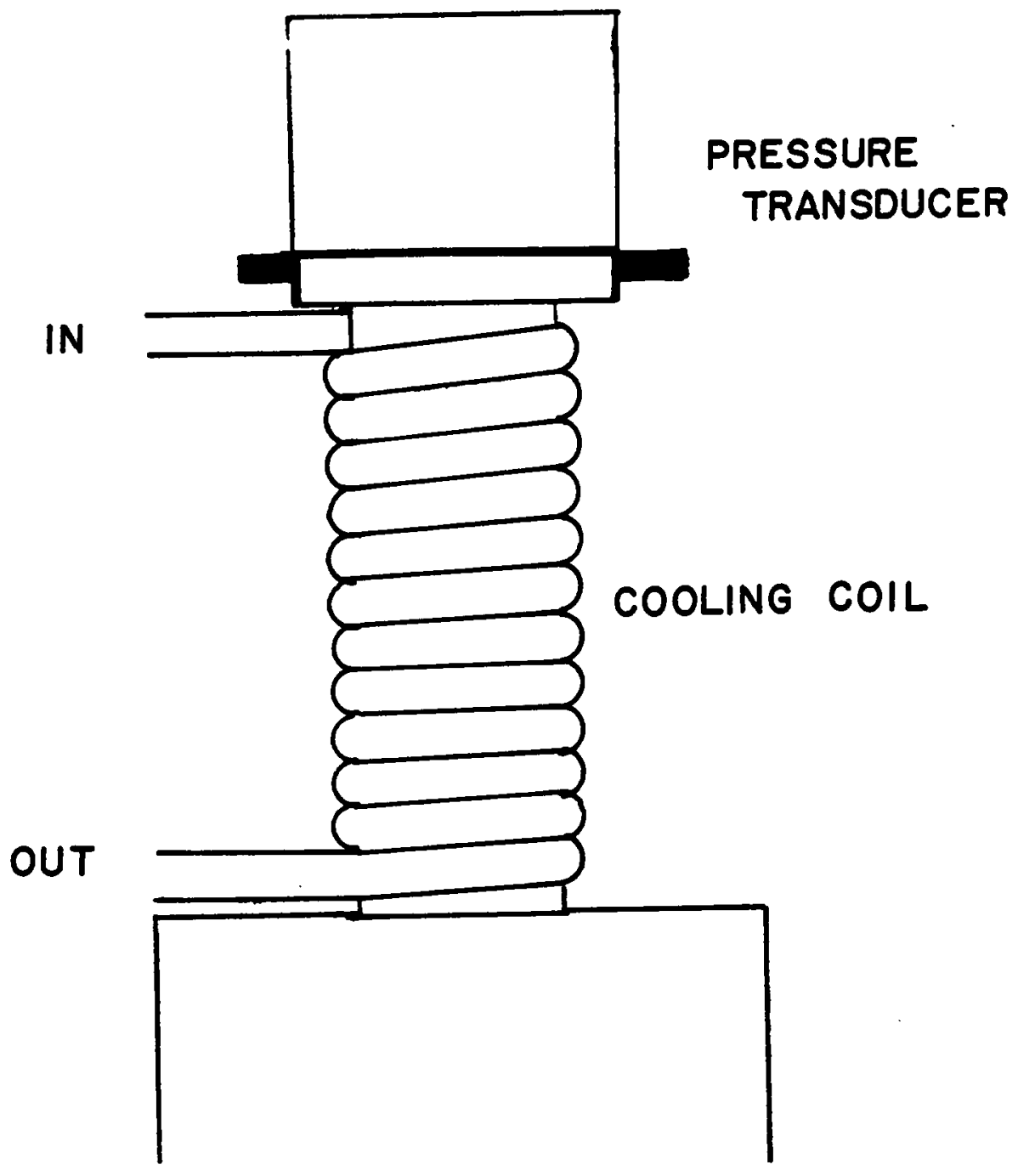

Figure 8. Syringe pump reservoir showing external copper cooling coils to maintain $\mathrm{CO}_{2}$ as a liquid. 
the cylinder to the reservoir. When liquid $\mathrm{CO}_{2}$ was transferred through the dip tube to the cooled pump reservoir, the cooling decreased the amount of gaseous $\mathrm{CO}_{2}$ in the system. When the reservoir was pressurized, the liquefied $\mathrm{CO}_{2}$ was denser at $0^{\circ} \mathrm{C}$ and less compressible. This allowed more rapid pressurization and increased the volume of liquid $\mathrm{CO}_{2}$ available for use by about 108. When the reservoir was pressurized from 800 p.s.i. (cylinder pressure) to 1100 p.s.i., $45 \mathrm{ml}$ of liguid $\mathrm{CO}_{2}$ was obtained while only $40 \mathrm{ml}$ was obtained at room temperature.

$\mathrm{CO}_{2}$ was pumped as a liquid through transfer tubing (1/16" stainless steel) to a three way, bottom vent high pressure valve (Varian, Walnut Creek, CA). This valve was used to release the pressure built up in the system during a gradient pressure program and to return the system to an initial starting pressure.

The $\mu L C 500$ pump provided operation in either a constant pressure or constant flow mode. The constant pressure mode was controlled by a pressure transducer mounted at the top of the reservoir and pressure was maintained by matching an input voltage set on the instrument to the output voltage of the transducer. This system was modified by the attachment of an external control circuit to the control ports on the back of the unit. This external controller provided for either normal (isobaric) operation or for linear pressure programming by inputting a ramp voltage into the pump control circuit. A schematic of the circuit is shown in Appendix A. The circuit linearly increased the pressure at the pump until the pressure reached a maximum pressure value and then 
held that pressure until reset. The maximum pressure setting was easily changed and was normally maintained at 5000 p.s.i.

\section{Injection system}

The injection system should introduce a reproducible, narrow band of material onto the analytical column at an appropriate concentration for chromatographic separation and detection. In gas chromatography, the sample is introduced with a syringe through a polymeric septum and transferred as a vapor onto the analytical column. The use of this technique for SFC is impractical due to the high operating pressures. Injection through a septum is practical only up to about 100 p.s.i. and then the septa begin to swell in supercritical $\mathrm{CO}_{2}$ and leak badly when pierced. HPLC injectors are designed for pressures up to 5000 p.s.i. and introduced a fixed volume of sample contained in a sample loop. The sample is introduced into the sample loop of the valve in the load position. When the valve rotor is turned to the inject position, the loop is brought in line between the pump and the column and the mobile phase transports it onto the column. This approach is suitable for SFC and was used in this work. However, the majority of HPLC sample valves are equipped with 5-20 $\mu \mathrm{L}$ sample loops and this is too great a quantity to introduce onto a capillary column. The smallest possible valve should be used and the sample may still need to be split in order to decrease the amount of sample loaded on the column. The splitter also serves to decrease the tailing of the sample and solvent peaks. By increasing the flow through the injector, the sample is quickly flushed 
and exponential dilution problems associated with low inlet flows are avoided.

In the initial design, a $0.5 \mu$ loop was used with an inlet splitter. The splitter was commercially available from SGE (Scientific Glass Engineering, Houston, TX) and the injector was a MACS 500 microbore HPLC injector (EM Science, Gibbstown, NJ). The splitter was modified in order to allow the split point to be located in the heated column oven while the sample valve was maintained at room temperature. A schematic of the modification is shown in Figure 9. The injection valve utilized an internal $0.5 \mu \mathrm{L}$ loop (Pigure 10 ) to contain the sample. Filling the loop was accomplished by flushing the loop with a syringe containing a solution of the material to be injected. To connect the column to the splitter, it was found that the best results were obtained with the front end of the capillary column located approximately $2 \mathrm{~mm}$ below the split point. Location of the column at or above the split point was found to result in increased band broadening. The split restrictor was a $25 \mathrm{~cm}$ length of $25 \mu \mathrm{m} \mathrm{I.D.} \mathrm{fused} \mathrm{silica}$ capillary. The restrictor length was varied to change the split flow. Normally, the split ratio was maintained at $20: 1$.

This design worked well, but the large split ratio resulted in increased solvent consumption and high flow rates at the pump. In order to decrease these factors, a smaller $(0.1 \mu \mathrm{L}$ ) valve (Valco model CI $4 \mathrm{~W}$ 0.1, Houston, TX) was obtained from Chrompack (Bridgewater, NJ). This valve performed satisfactorily with reduced split ratios of about 2:1 commonly being used. 
MODIFIED

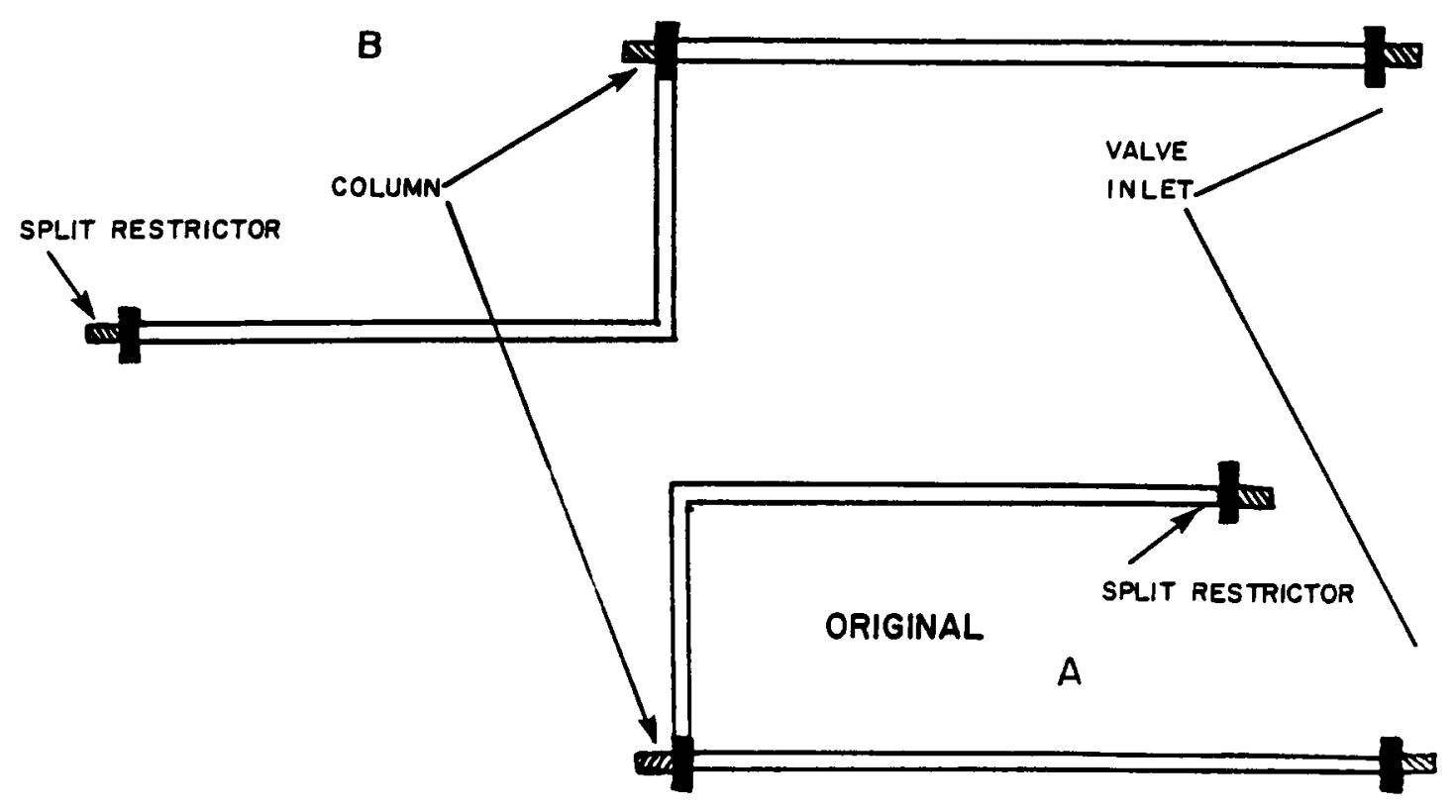

Figure 9. SPC inlet splitter showing modification to permit easier insertion through P.E. Sigma 2000 oven wall. Figure $A$ is original design. Figure $B$ is the modified version. 


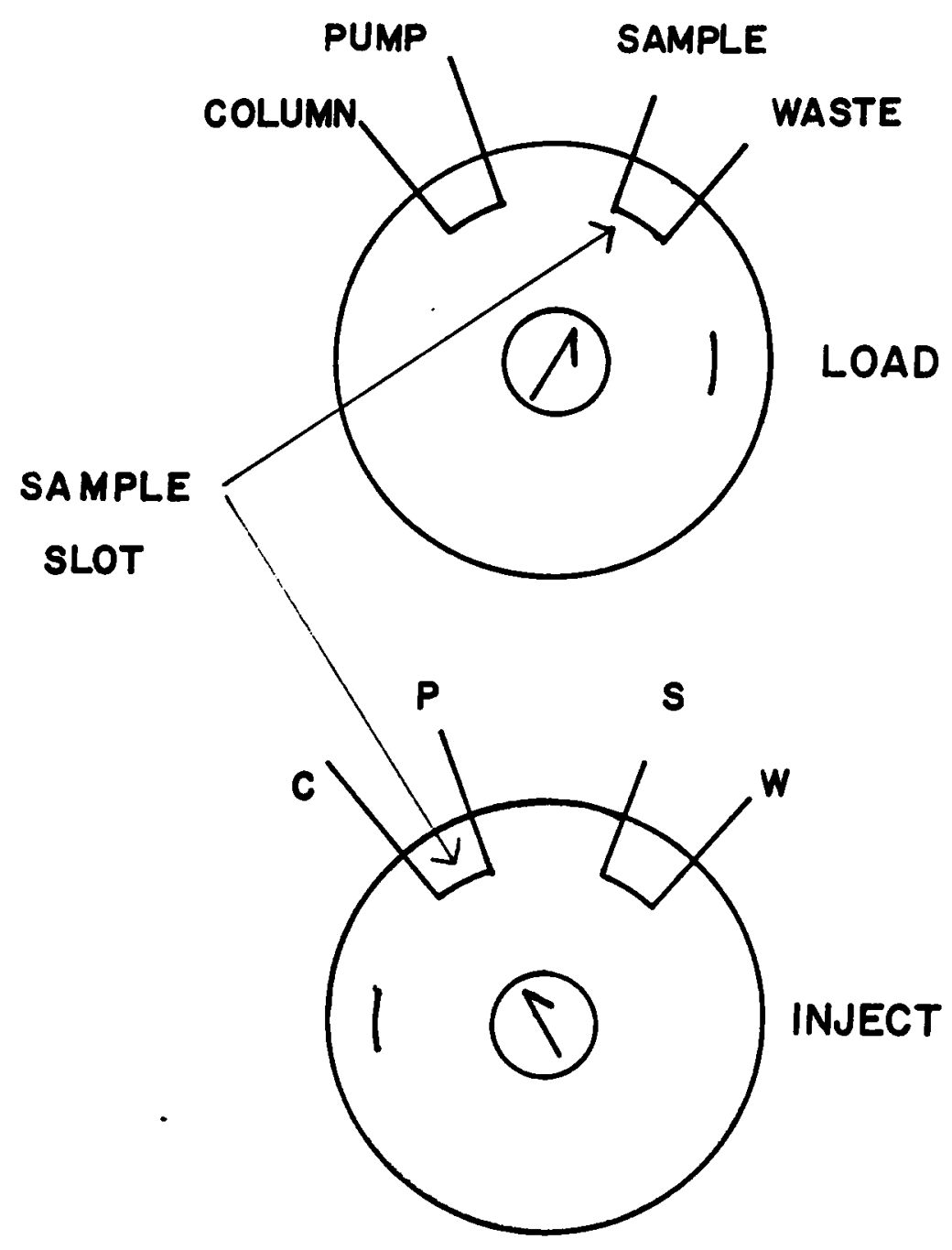

Figure 10. Internal loop sample value for submicroliter injection at high pressures. 
Any split of sample results in decreased material reaching the column and a subsequent loss in sample detection limits. A split is generally used to perform two functions. First, the splitter prevents the sample from overloading the column when more concentrated samples are injected. Second, the splitter allows the injection valve to be flushed with mobile phase at a high flow rate to quickly empty the loop and prevent solvent peak tailing caused by the exponential decay in concentration of solvent in the injector loop.

To dispense with the splitter, the samples being analyzed must be sufficiently dilute so that the analytes do not overload the column. The sample loop must be small and well designed so that the sample is deposited as a narrow band in the column and the loop should be well swept by the mobile phase to prevent peak broadening due to dead volume. In order to evaluate the possibility of direct injection onto a 100 $\mu \mathrm{m}$ i.d. capillary column, a direct connection was made into the $0.1 \mu \mathrm{L}$ sample valve. To minimize dead volume in the inlet, the column was positioned with the tubing insert against the valve rotor (Figure 11). The results obtained with and without the splitter are shown in Figure 12. No increases in sample band width resulted and no extreme tailing of the solvent was found. Sample washout from the injection loop was rapid. A c-24 normal alkane in dichloromethane was injected repeatedly and the area of the resulting peak measured. During this test the valve was left in the "inject" position. The same sample was injected and the sample valve was returned to the "load" position after 10 seconds. No decrease in area for the c-24 hydrocarbon was observed, indicating that 


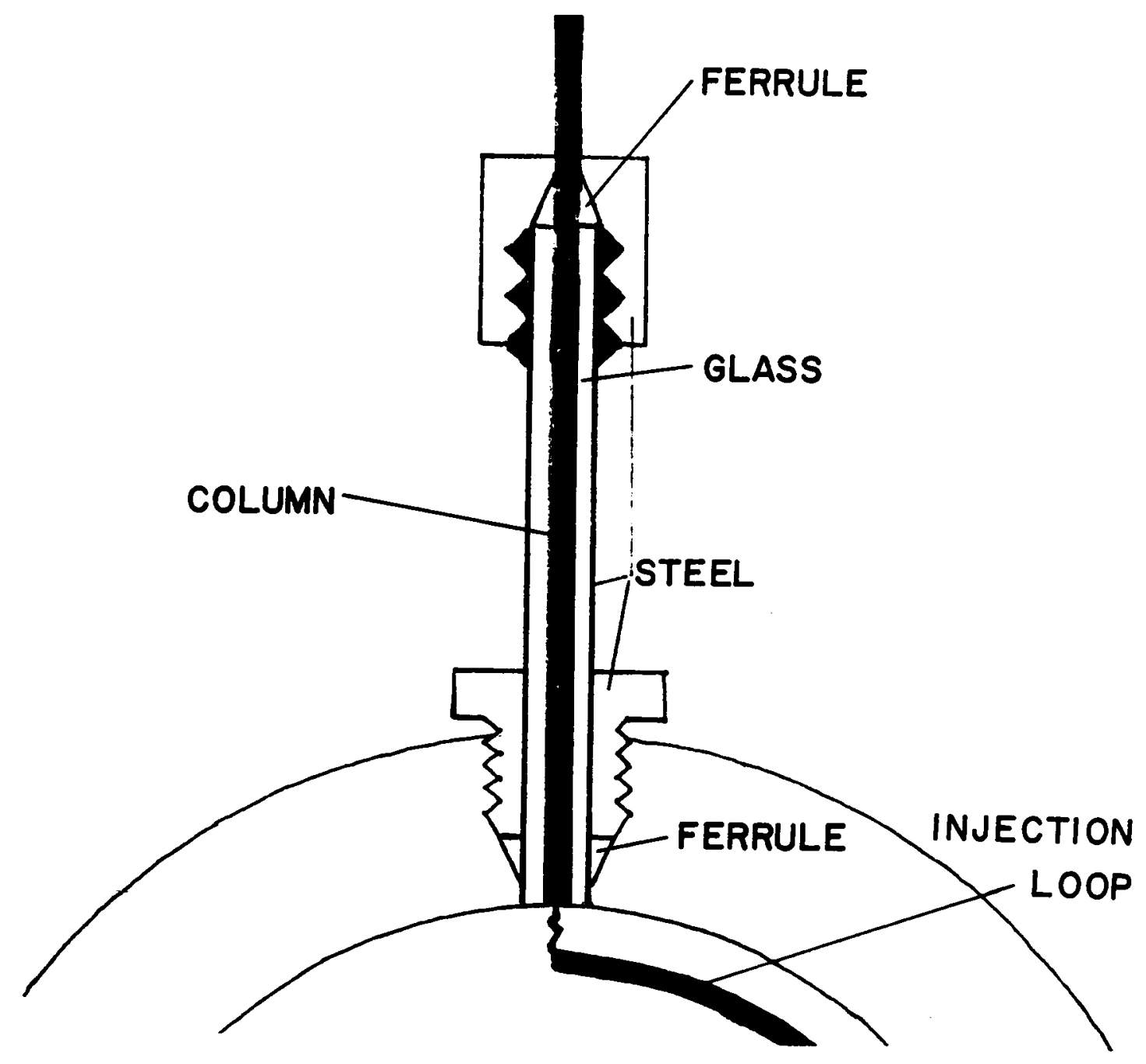

Figure 11. Illustration of proper seating of column to injection valve for SFC direct injection. 

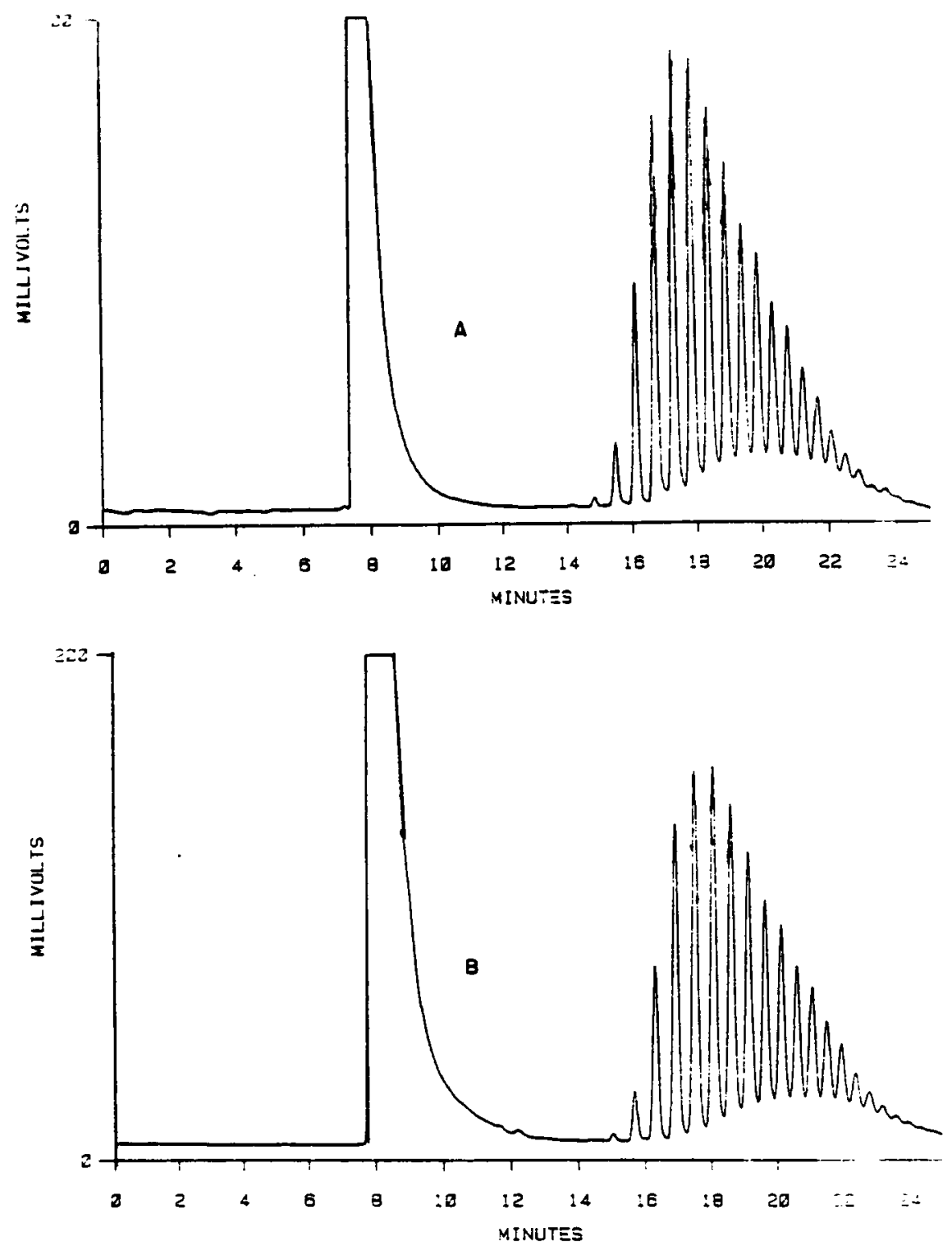

Figure 12. Paraffin sample with (A) split injection and (B) direct injection. Temperature $100^{\circ} \mathrm{C}$. Pressure programmed from 1100 p.s.i. at 125 p.s.i./minute. 
sample transfer to the column had already occurred. It was calculated that 10 seconds in the "inject" position allowed the loop to be flushed 20 times with the mobile phase. It should also be noted that even though the tetracosane has no appreciable solubility in $\mathrm{CO}_{2}$ at the injection density, there was no precipitation of sample in the loop. The sample presumably was transported onto the column while still dissolved in the dichloromethane. This observation suggests the use of the sample inlet system for on-column concentration.

As was shown earlier in Figure 2, the sample in SFC can be "frozen" on the column by injection at densities below that necessary for sample migration. This characteristic can be used to concentrate dilute analytes on the column and hold them until subsequent elution. This effect can be used to extend the application of SFC to trace components at low concentrations.

In order for successful sample concentration to occur, three criteria must be met: 1) the sample must be out of the loop before the next injection is made; 2) the sample must be deposited at a density where it is not soluble enough to migrate; and 3 ) the sample must not be volatile enough at the column temperature to migrate. The first condition is met by the experimental design, the latter two can be addressed by proper choice of operating conditions. Figure 13 shows the injection of $50 \mathrm{ppm}$ of tetracosane directly onto the analytical column. This corresponds to $5 \mathrm{ng}$ of the alkane on-column. Figure 14 shows five injections of $10 \mathrm{ppm}$ of tetracosane with the $0.1 \mu \mathrm{L}$ valve to give a total of $5 \mathrm{ng}$ on-column. The integrated peak areas are the same and no 


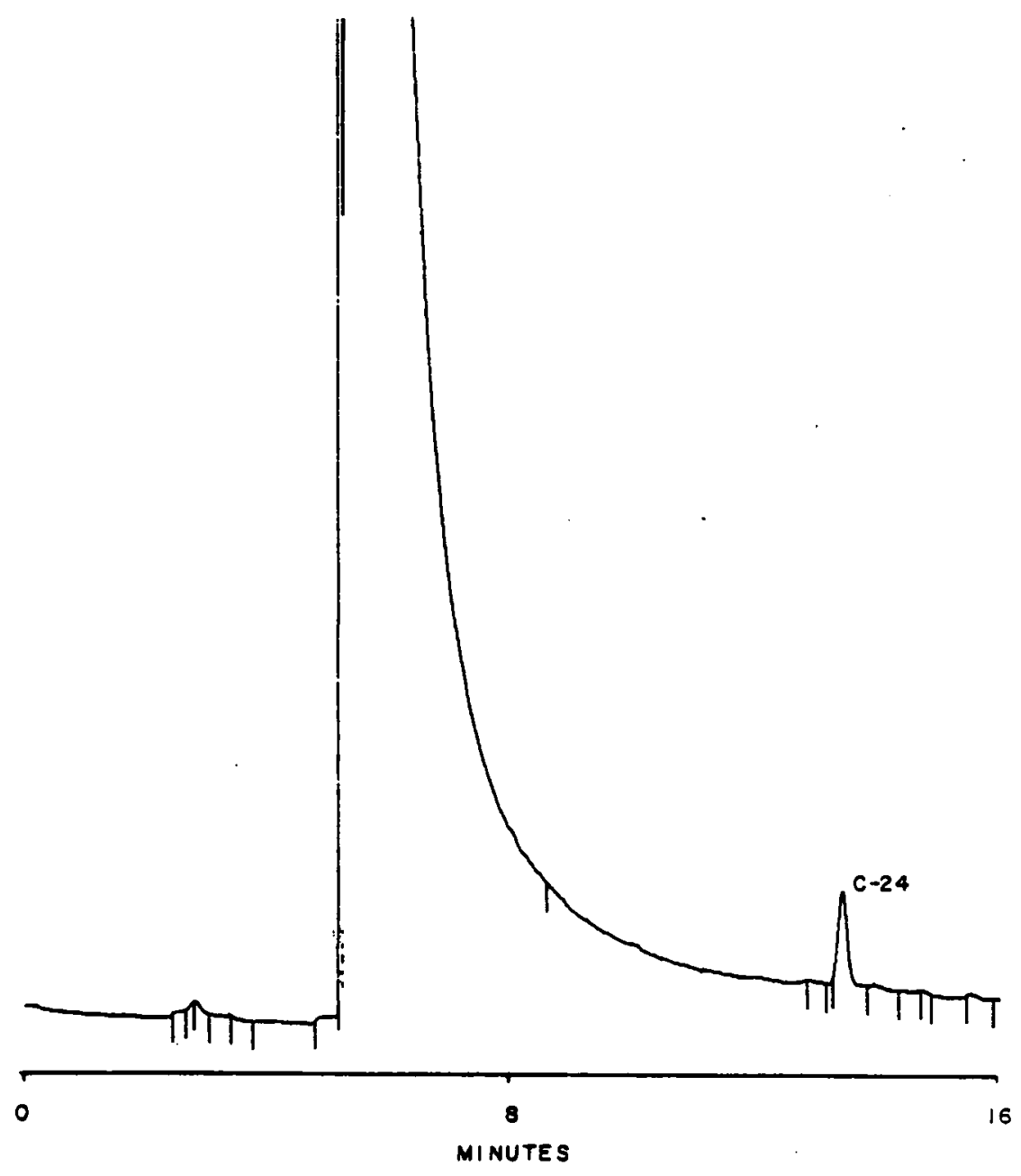

Figure 13. One $0.1 \mu \mathrm{L}$ injection of $50 \mathrm{ppm}$ of a tetracosane in dichloromethane. Temperature $150^{\circ} \mathrm{C}$. Pressure programmed from $1100 \mathrm{p.s.i.} \mathrm{at} 125 \mathrm{p.s.i./minute.}$ 


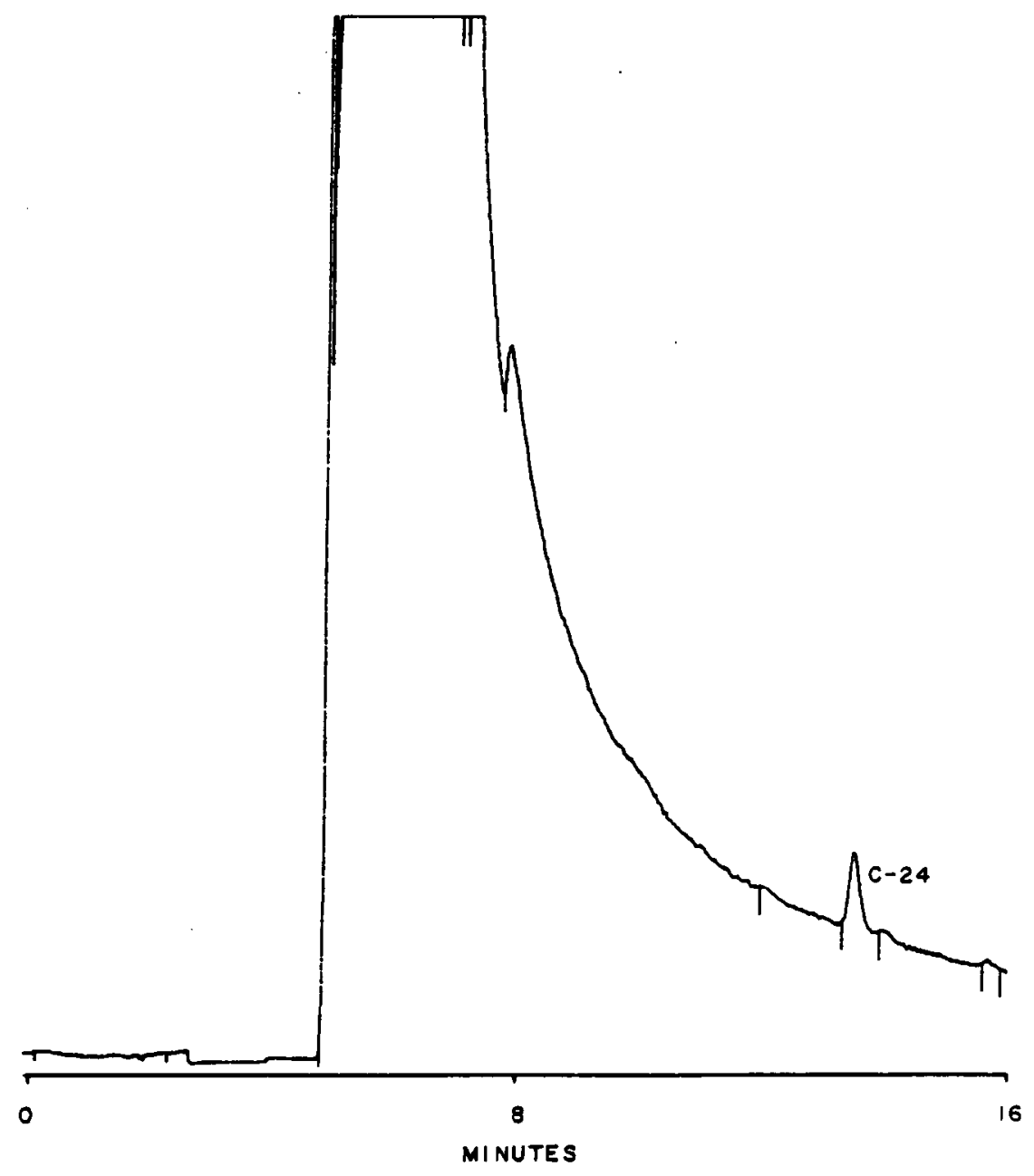

Figure 14. Five consecutive $0.1 \mu \mathrm{L}$ injections of a $10 \mathrm{ppm}$ of a tetracosane in dichloromethane. Temperature $150^{\circ} \mathrm{C}$.

Pressure programmed from 1100 p.s.i. at 125 p.s.i./minute. 
additional band broadening of the analyte is observed. The greater amount of solvent injected in Figure 14 is seen by the increased width of the solvent peak. A 30 second isobaric period was used initially on both runs before programing was started. An increase in this period would allow the solvent to be eluted well before the analyte.

The effect of injection at a density which permits sample migration is seen in Figure 15. Conditions are the same as Figure 14 but the initial pressure has been increased to 2500 p.s.i. Under these conditions band broadening caused by the individual injections can be seen.

In considering this repetitive injection technique for analyte concentration, the point must be addressed that if five consecutive 0.1 HL injections allow decreased concentration levels to be analyzed, why not inject once with an $0.5 \mu \mathrm{L}$ sample loop. Figure 16 shows the effect of 10 ppm of tetracosane injected with an $0.5 \mu \mathrm{L}$ loop directly on the column. Other than injection loop size, all conditions are identical. The tetracosane was found to elute with the solvent front and the small peaks located on the large solvent tail are solvent impurities. The disastrous effect of the $0.5 \mu \mathrm{L}$ loop can be explained by the necessity to sweep out the comparatively large loop volume which causes the severe tailing, and more importantly, the effect of the instantaneous injection of a large solvent volume.

\section{Column}

The experimental work with capillary columns and the results obtained will be dealt with in a later section. At this point, the 


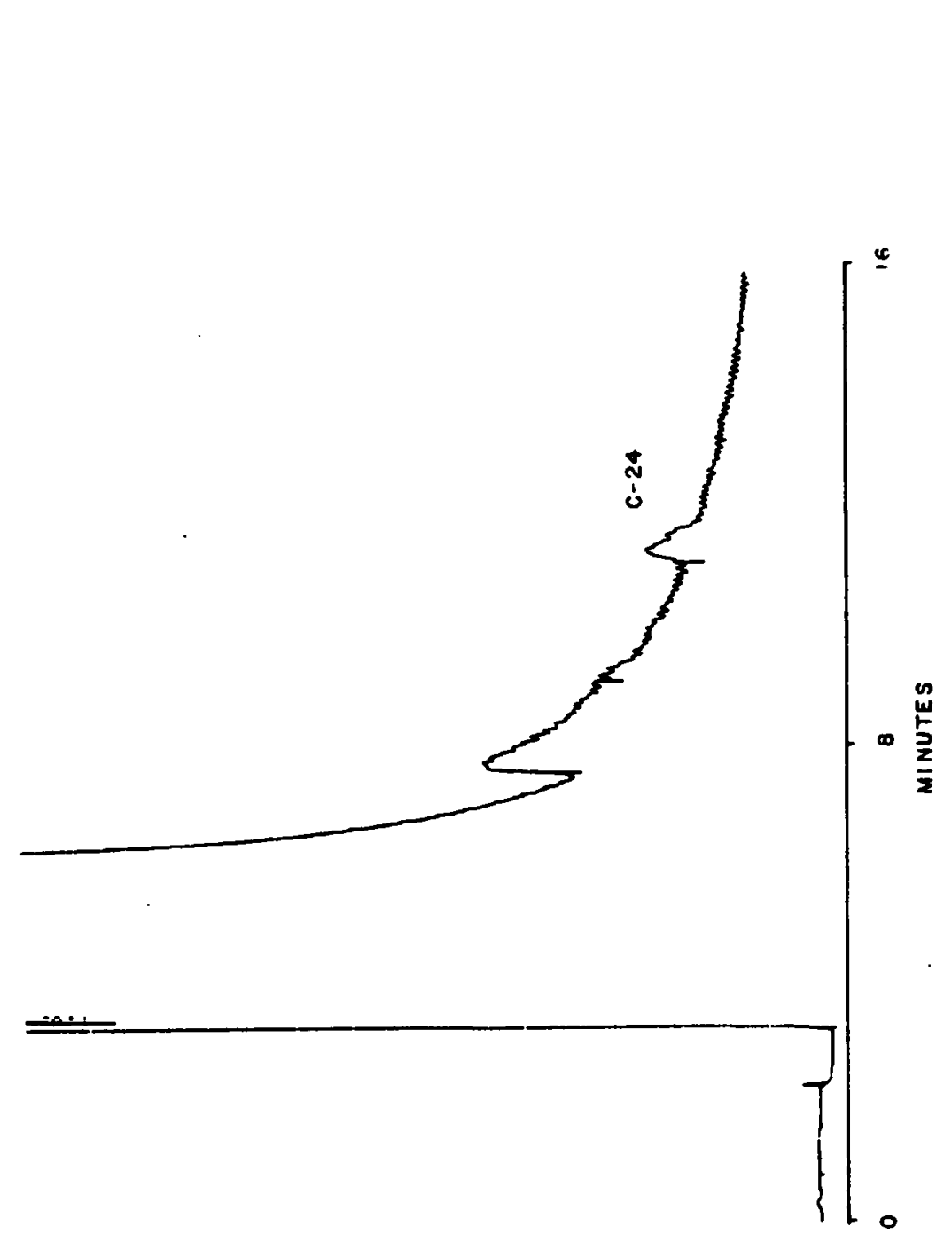

Е్

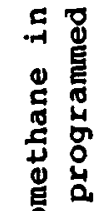

o

ํ.

롱

ठ

.

¿

骂

8

号

ญ

- 8

แ

ह

谷 。

어 है है 茨 공

号

苞茟

.

号

솔뭉

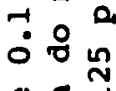

구

声节

ว

o

ठํ.

๑ 응

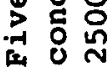

苗 

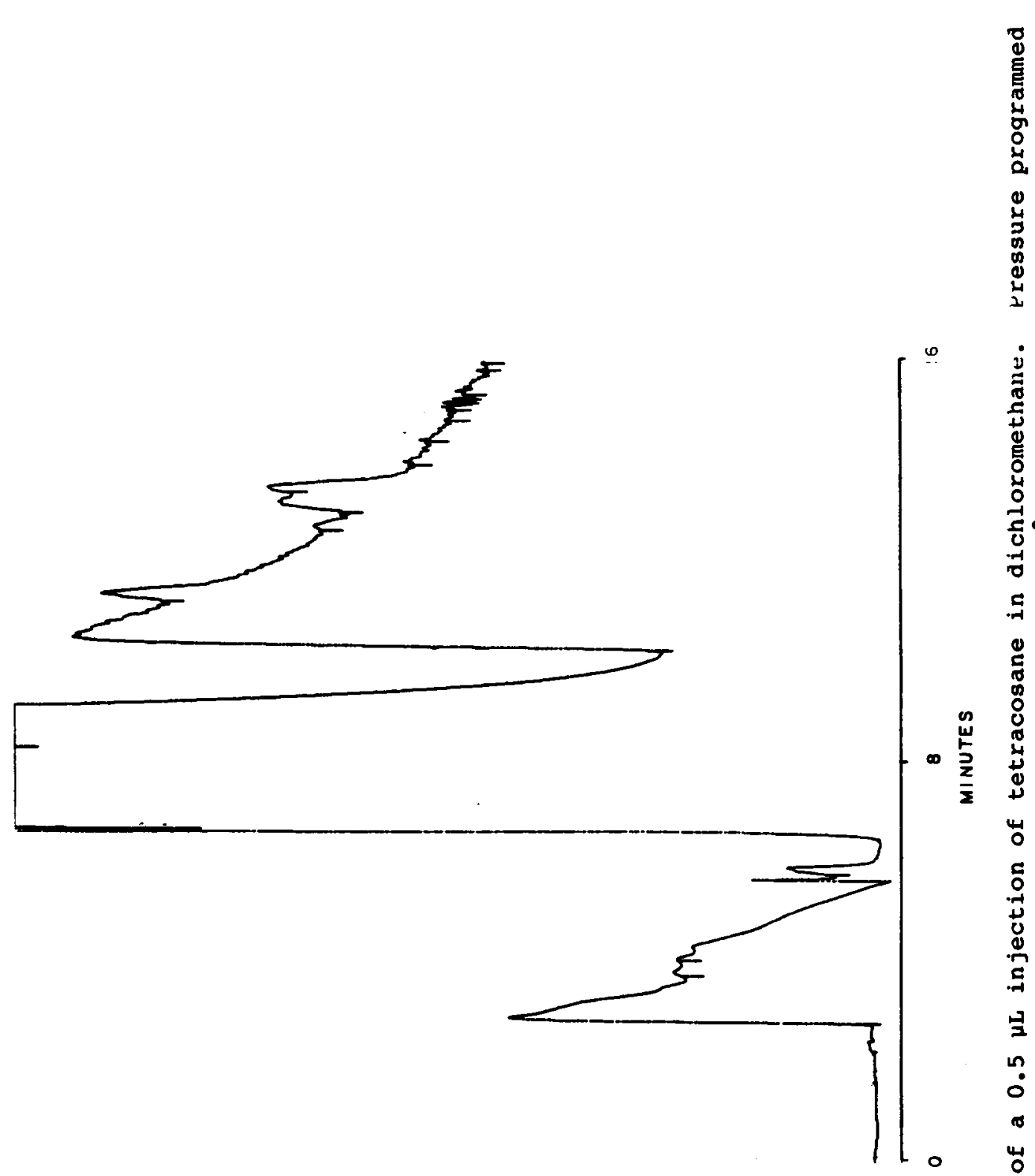

נِ

ن

동웅

거

$\rightarrow$

步

गै

- 8 일

赵

$+0$

岁 芫

도

نे

o

I

보ำ

ก

$\infty$

虫 的

in 0

용

出

点

点 
POLYIMIDE

FUSED SILICA

Figure 17. Cross-section of a capillary SFC column. 
column specifications will be dealt with in general terms. The column is an open tube coated with a thin, uniform film of polymeric material, the stationary phase (Figure 17). This material must be extensively cross-linked to render it insoluble in the supercritical $\mathrm{Co}_{2}$. In addition, the column must be capable of withstanding high internal pressures (up to 6,000 p.s.i.). Since this ability is a function of column material and inside diameter (wall area), the use of small, strong tubing is indicated. Experimental use has shown fused silica columns with internal diameters of $0.1 \mathrm{~mm}$ and $0.25 \mathrm{~mm}$ are capable of withstanding these pressures without failure. A $0.1 \mathrm{~mm}$ i.d. column was used at 7000 p.s.i. without failure, but operation at these pressures is limited by the pressure rating of the inlet valve. Internal diameters play an important role as is indicated by the report of a catastrophic failure of a $0.53 \mathrm{~mm}$ i.d. column when used for SFC. ${ }^{145}$

The column is enclosed in a gas chromatographic oven (Perkin-Elmer Sigma 2000, Norwalk, CT) to maintain a constant temperature during the analysis. One advantage of the sigma 2000 is the presence of removable ports on the sides of the oven. This access allows the use of the instrument for SFC without the need to drill holes through the oven wall.

\section{The Column Detector Interface}

One of the more difficult problems with SFC is the need to connect the chromatographic column where separation occurs to an instrument where detection and quantification can occur. For spectrophotometric 
detectors, this difficulty can be overcome by the use of high pressure flow cells and downstream restriction devices. For other detectors, which operate at atmospheric pressure, a more complex problem is presented.

For FID's and related detectors, several criteria must be met for successful operation. The interface between the column and the detector must maintain the column at a high pressure and a low linear velocity. In addition, the interface should provide a flow of sample containing $\mathrm{CO}_{2}$ at a temperature and flow suitable for the detector. These requirements are met by the use of a mass flow restrictor between the column and the detector. A restrictor provides a slow leak of material into the detector while maintaining a high (up to 5500 psi) column pressure. The geometry of the restrictor is of vital importance. Unswept, or dead volume should be minimized in the connection to the capillary column. Several designs are available for connecting capillary columns together in $\mathrm{GC}$, but the best results were obtained with a design from SGE (Scientific Glass and Engineering, Austin, TX). This vitreous silica union (VSU 003) is glass lined stainless steel with external nuts to connect the column and the restrictor.

The best ferrules for the connections were found to be made of a polyimide material. Graphite ferrules were also used successfully but they were not as robust and tended to deform badly when reused. The internal diameter and the length of the restrictor determines the flow rate through the column.

Several restrictor styles have been proposed and each has met with some success. An ideal interface would be a point restrictor. Since 


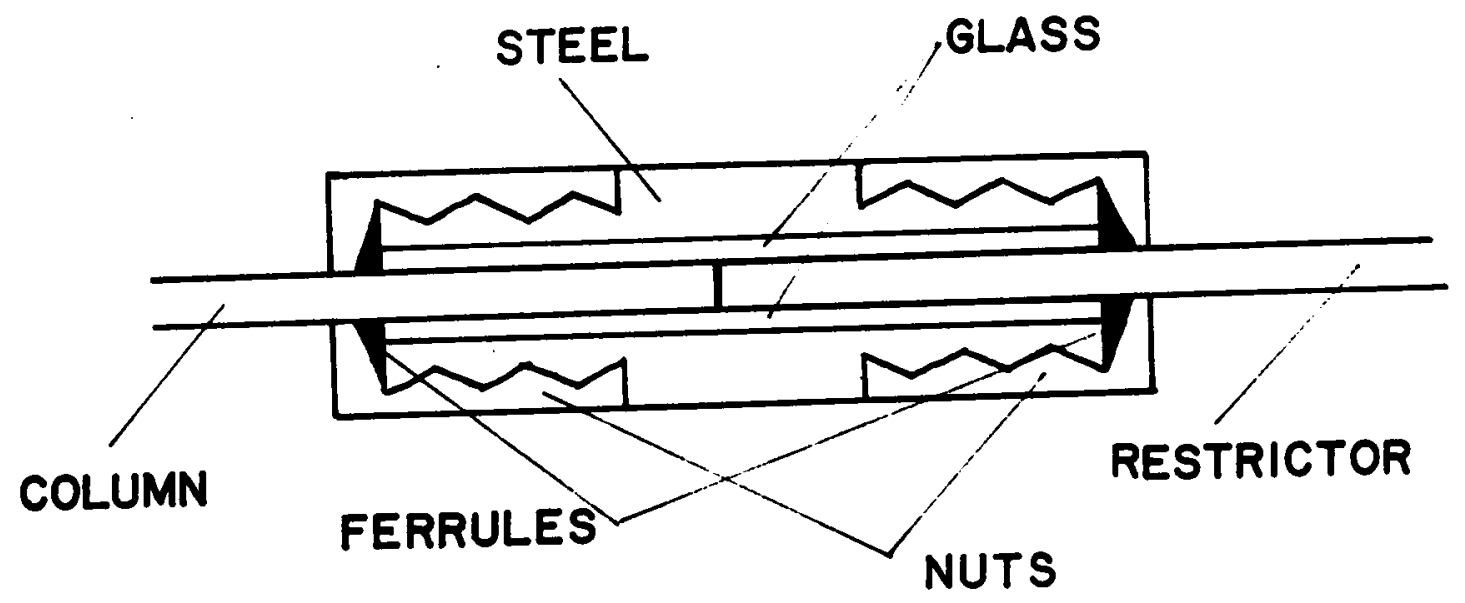

Figure 18. A zero dead volume connector for SFC detector interfacing. 


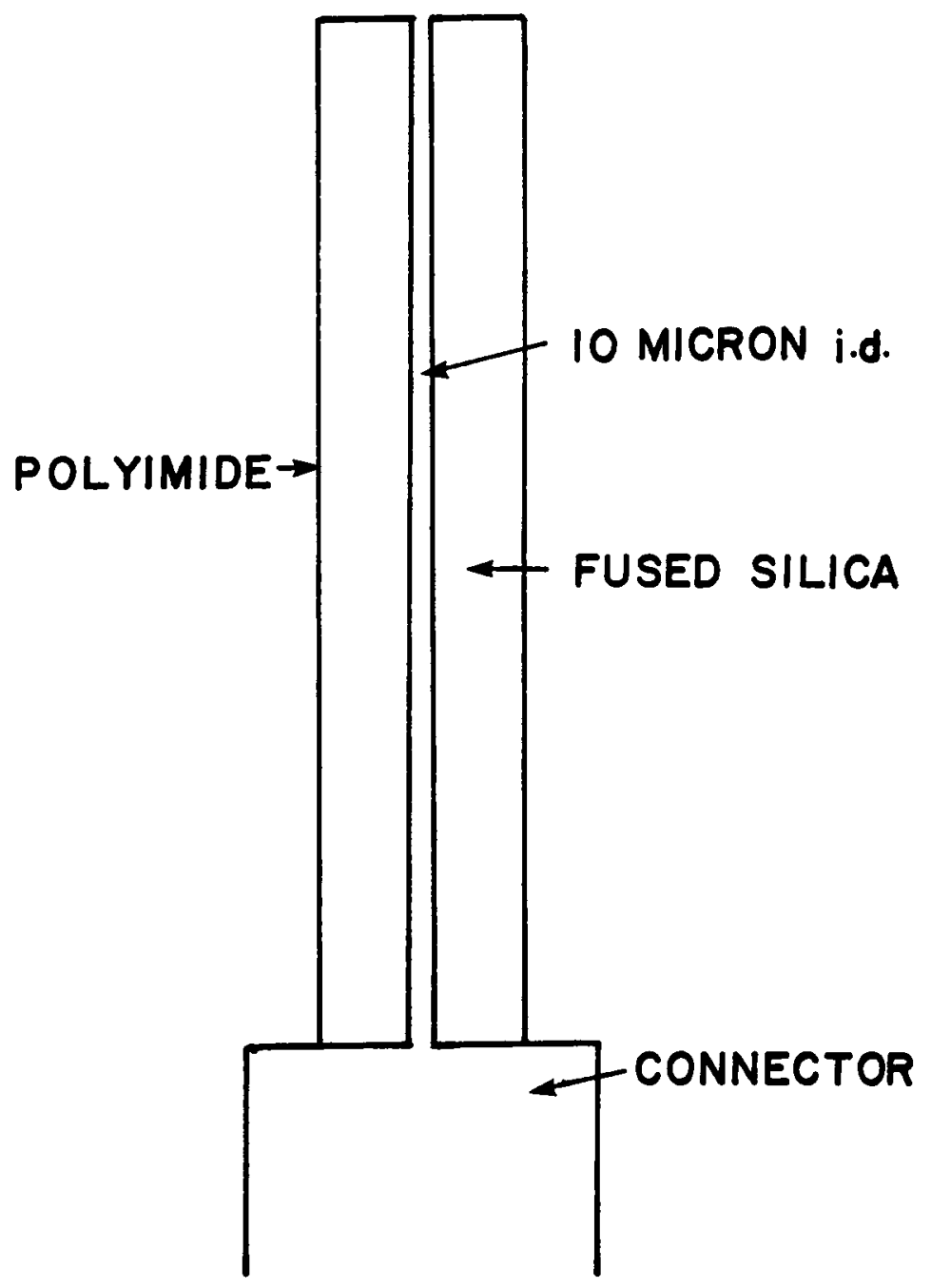

Figure 19. SFC/FID linear restrictor. 
81

the density and solvent power of the supercritical fluid is related to its pressure, a long restrictor would provide a gradual pressure drop allowing the sample to precipitate out of solution. A short restrictor minimizes this precipitation due to the high velocity in a small internal diameter chamber. Another factor affecting the design of the restrictor is the realization that small orifices plug easily. A restrictor must provide for reasonable flows at the working pressure without plugging.

Linear restrictors of fer the advantages of resistance to plugging and good handling characteristics. It was found that spiking with these restrictors could be minimized or eliminated by maintaining a hot $1350^{\circ}-$ $400^{\circ} \mathrm{C}$ ) detector and selecting restrictor length and internal size carefully. For example, a 5 um i.d. restrictor exhibited increased spiking compared to a $10 \mu \mathrm{m}$ i.d. restrictor. A $10 \mu \mathrm{m}$ restrictor was found to give little or no spiking when correctly installed in the flame tip and well heated. Since spiking was not a problem for this design, due in large part to the detector design of the Perkin-Elmer Sigma 2000 FID, linear restrictors were used exclusively.

\section{Detector}

The detection and quantification of an analyte is dependent on the nature and operation of the detector chosen. A detector should satisfy several criteria. The detector should be linear over a wide range, compatible with the mobile phase, sensitive for the analyte at low levels, and stable over a period of time. Detectors can be classified into two groups, universal detectors and selective detectors. A 
universal detector responds to all substances while a selective detector is responsive only to specific sample types and exhibits little or no sensitivity to other species.

While selective detectors are useful for detection of low levels of analytes or for picking out specific compounds from a complex matrix, universal detectors have much greater utility for general analyses. In selecting an instrumental design, it was decided to work with a fairly universal detector, the flame ionization detector, rather than a selective detector.

The FID has several attractive features. It is relatively inexpensive, rugged, is widely used in GC and has an excellent response for organic compounds. The principle of operation involves the combustion of the analyte in a hydrogen flame. The flame is located between two electrodes which are held at different potentials. The ions migrate toward a collector electrode in a polarizing electric field. The small current which is caused by ion migration is amplified and fed to a strip chart or integrator. Only compounds that are ionizable by a $\mathrm{H}_{2}$ /air flame can be detected. Carbon dioxide is not ionizable in the $\mathrm{B}_{2} / \mathrm{air}$ flame and so provides a low background for sample detection. The Perkin-Elmer F.I.D. used in this work has several advantages over the conventional F.I.D. This detector has been designed for capillary GC applications and has a low internal volume which needs no additional make-up gas. In addition, the flame gases are pressure rather than flow controlled with the hydrogen mixing with the carrier flow and increasing the transport rate through the flame tip. 
83

The linearity of the Perkin-Elmer FID for SFC was good, with a working range of $1 \mathrm{ng} /$ peak to $500 \mathrm{ng} /$ peak. As is shown in Figure 20 at higher analyte concentrations, the signal/amount weight of analyte decreases. Operation at this high concentration would be impractical since column overloading begins to occur at lower levels as is indicated by the "fronting" peak shown in Figure 21. This peak distortion indicates that this sample should be diluted before analysis. The limit of detection (LOD) is stated as being that amount of material which can be significantly and reliably distinguished from background noise and is defined as a signal three times the noise level. In order to maximize the sensitivity of the FID, several steps were taken. The $\mathrm{CO}_{2}$ mobile phase was obtained in a highly purified form to minimize organic contaminants and the column was well conditioned to prevent stationary phase "bleed". In addition, scrubber columns filled with silica gel and $5 \AA$ molecular sieve were installed on both the $\mathrm{H}_{2}$ and air detector gases to decrease the possibility of contaminants in the detector which would increase the background ionization of the flame.

The sensitivity of the FID was optimized by manipulation of the $\mathrm{H}_{2}$ /air ratio. In Figure 22 the $\mathrm{H}_{2}$ flow rate was optimized by increasing the $\mathrm{H}_{2}$ pressure and flow. One notable difference is seen, in GC the $\mathrm{H}_{2}$ flow passes through a maximum and then the signal decreases upon addition of additional $\mathrm{H}_{2}$ flow, while in the SFC configuration the sensitivity and $H_{2}$ flow rates are proportional up to the limit of available pressure. 
84

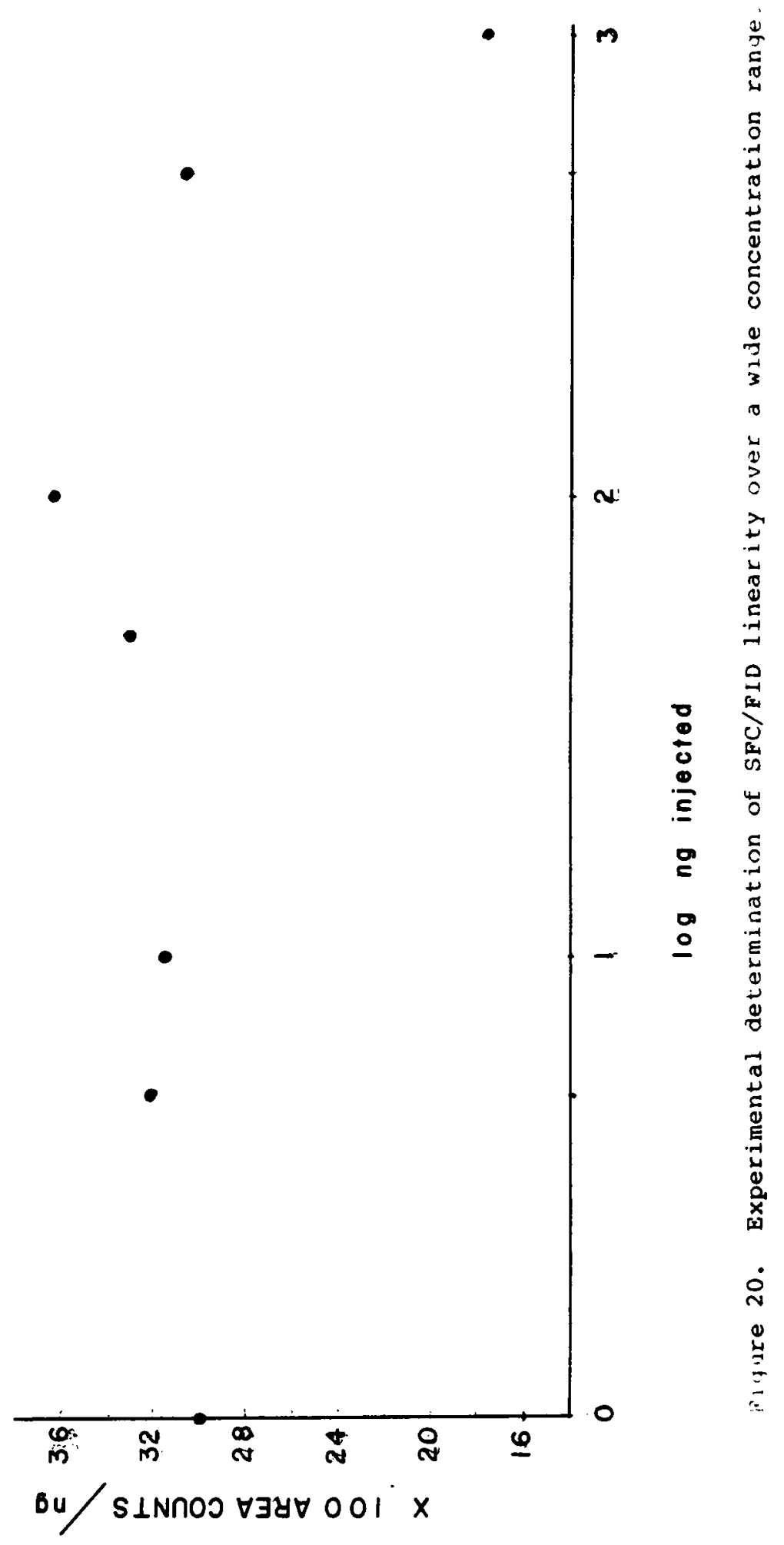




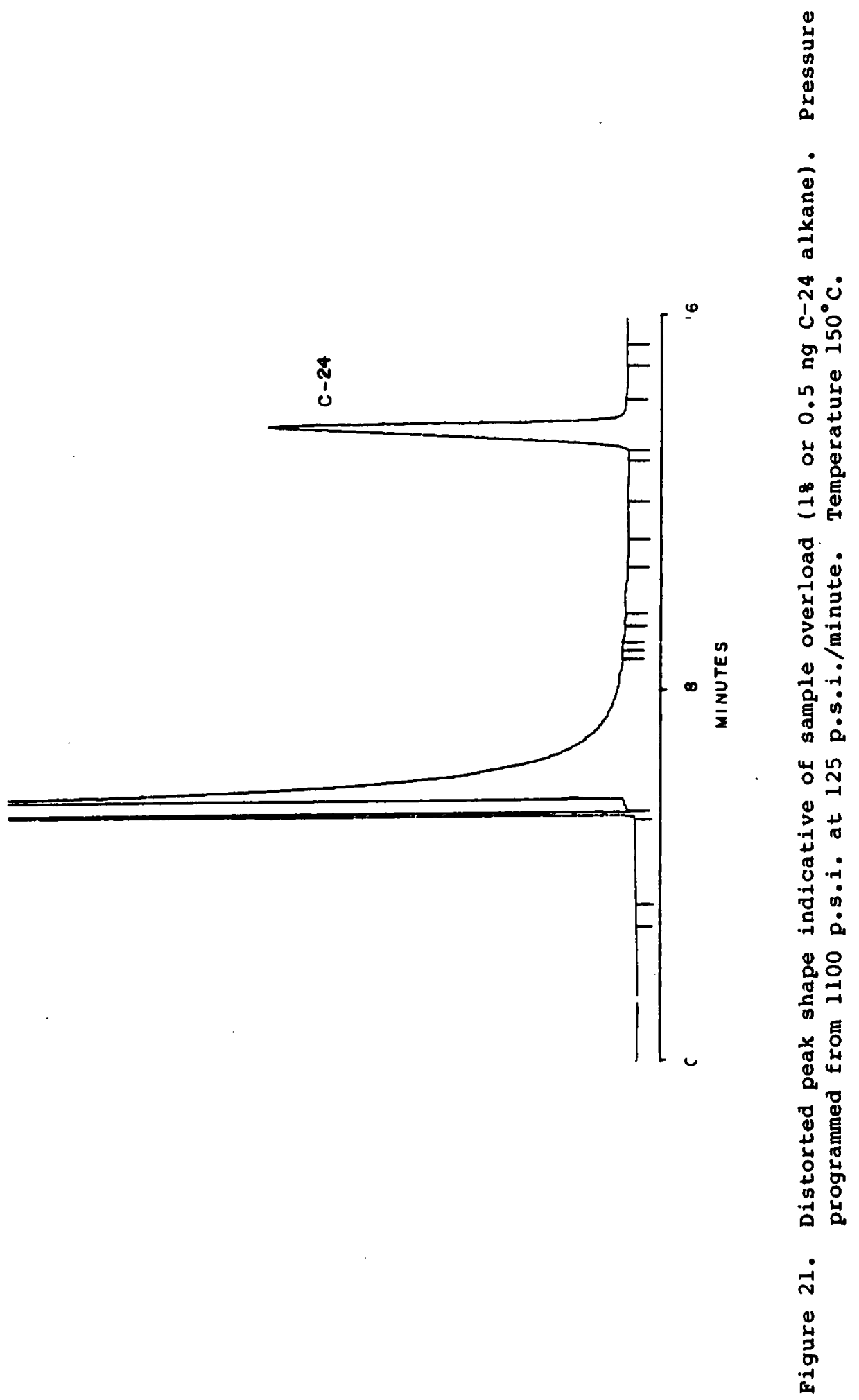




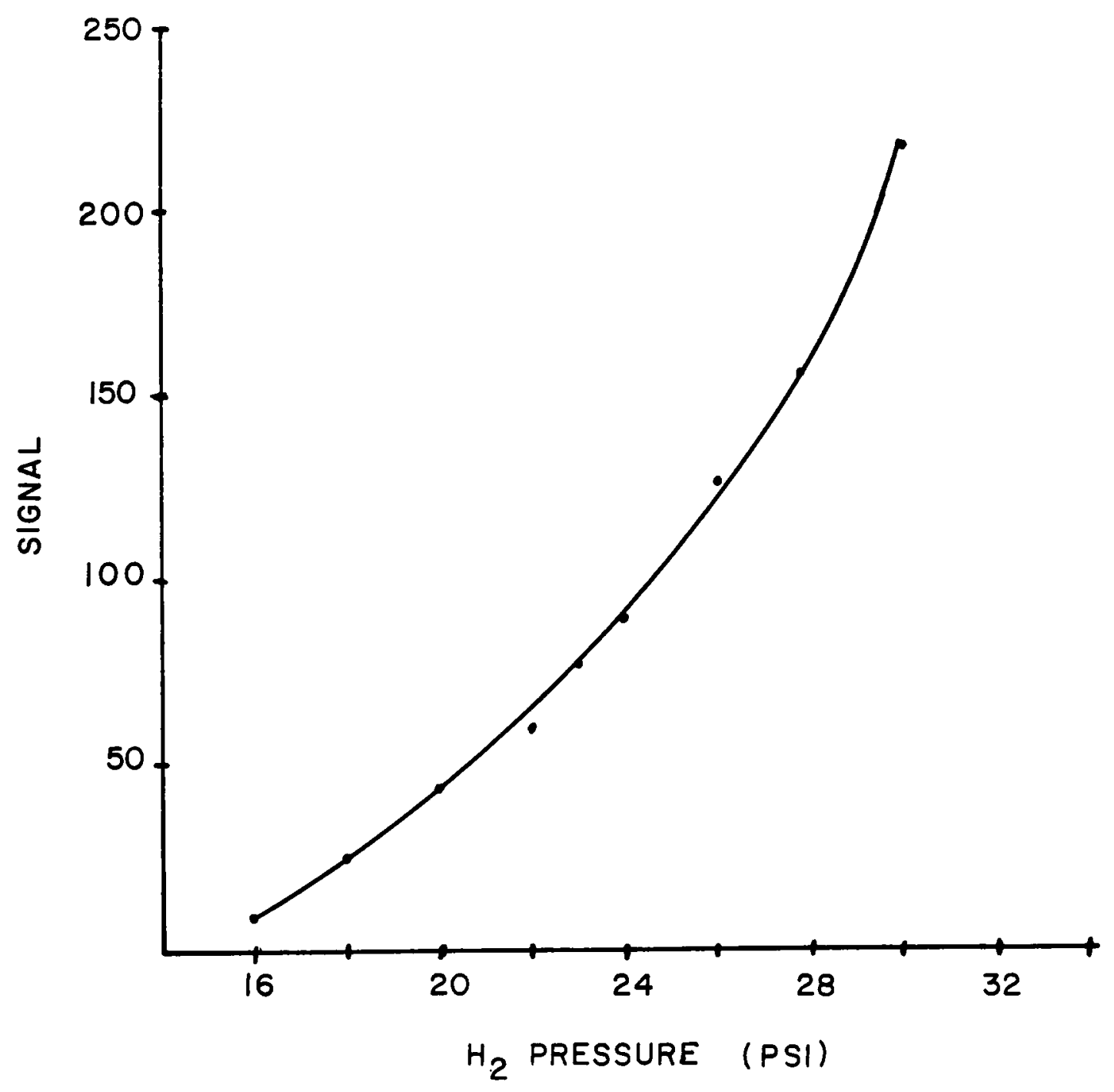

Figure 22. Effect of $\mathrm{H}_{2}$ gas pressure on FID sensitivity. 
These values were obtained by setting a column pressure of 2500 psi and a column temperature of $100^{\circ} \mathrm{C}$. At an air pressure of $26 \mathrm{psi}$, the $\mathrm{H}_{2}$ flow was varied and the signal measured. The increase in the baseline was measured in arbitrary units.

Following the determination of sensitivity dependence on $\mathrm{H}_{2}$ flow rate, the effect of air flow rate was measured. With the $\mathrm{H}_{2}$ pressure set at 30 psi, the air pressure was changed and the results are summarized in Figure 23. The dependence of sensitivity on air flow rate follows accepted behavior for GC and plateaus off at high pressures. The minimum detectable quantity with this optimized system was found to be $0.5 \mathrm{ng}$ which represents $3 x$ noise level. This corresponds to a relative concentration of $5 \mathrm{ppm}$ for an injection volume of $0.1 \mu \mathrm{L}$. (Eigure 24). 


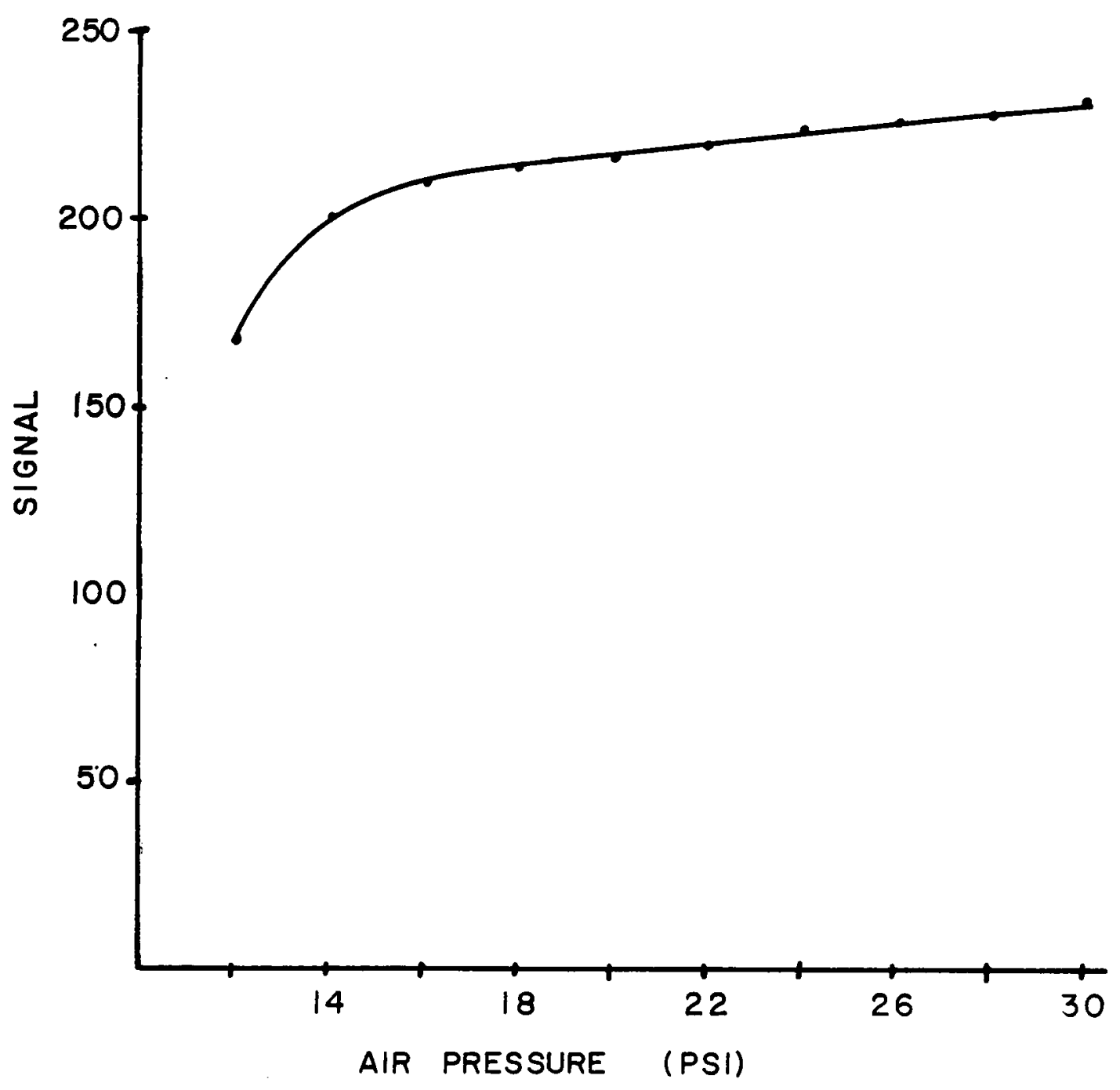

Figure 23. Effect of air pressure on FID sensitivity. 


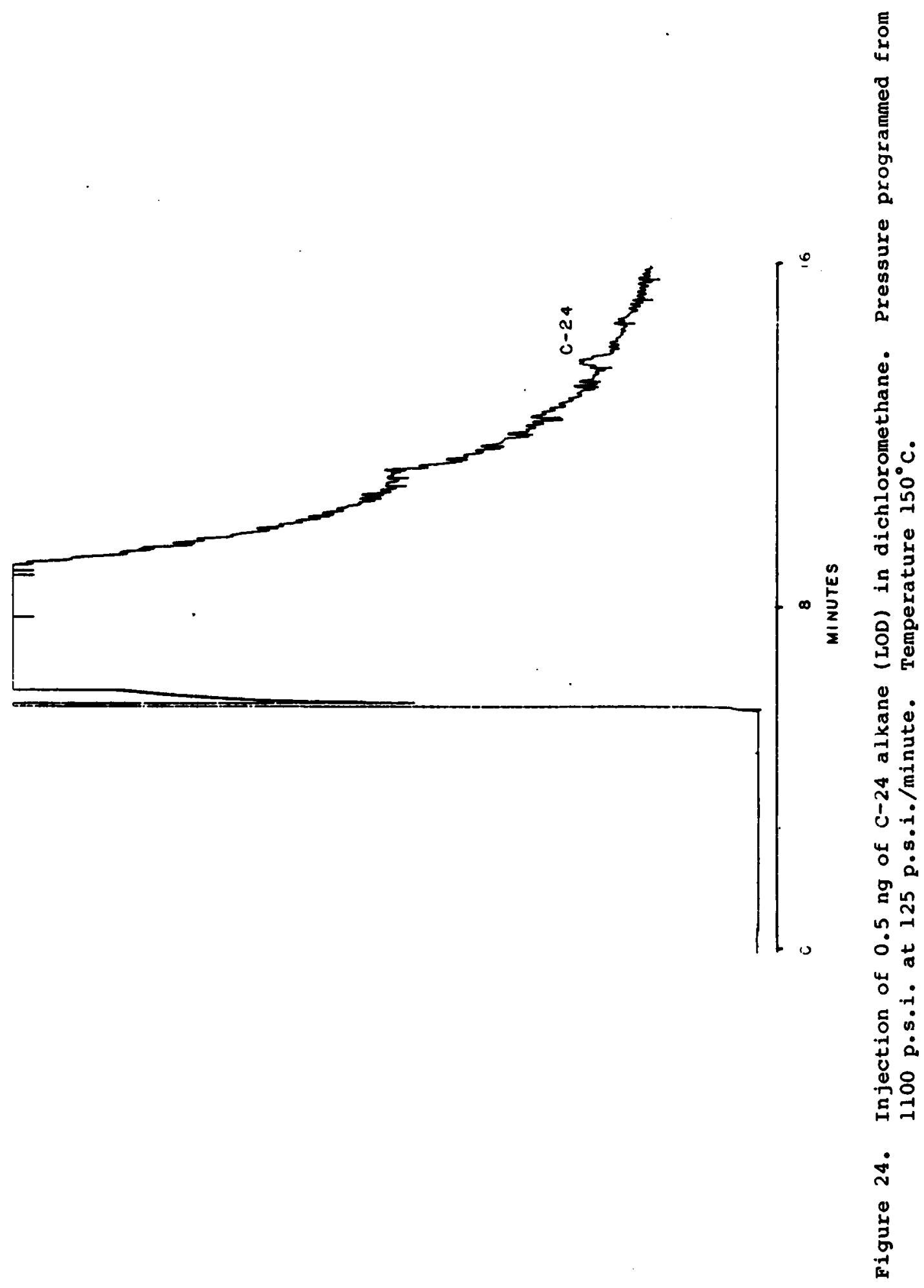


CHAPTER IV

CAPIILARY SFC COLUMN PREPARATION, COATING, AND EVALUATION

\section{Introduction}

The preparation of capillary columns for gas chromatography has been described as more of an art than a science. Although a better understanding of the coating process and the chemistry and physics which are involved has led to more rational and reproducible work, the complexity of the various steps in column preparation and the techniques needed to successfully make a good column require experience which can only be gained by trial and error.

The preparation of capillary columns for SFC is more complex than for GC. In addition to the requirements of a thin, uniform coating of polymer on the walls, the column must be extensively cross-linked to survive the continuous rinsing by the supercritical solvent. Also, the smaller internal diameters used in SFC are more difficult to coat and crosslink than the larger GC columns.

\section{Column Materials}

The material which composes the capillary column dictates the activity, efficiency, and handling characteristics of the resulting coated capillary column. Three materials are in common use for capillary GC columns, metal, glass and fused silica. Of these three, only glass and fused silica have been used for SFC, due primarily to the strong adsorption of analytes on metal columns. Both glass and fused silica have been shown to work well with SFC. They are both examples of 
silica glasses and differ primarily in the amount of impurities in the material.

The composition of the glass affects both the physical and chemical characteristics. Fused silica is a very pure silica which has been reported to exist in a randon network. 147 The lack of metallic impurities in fused silica results in an inert material which gives decreased adsorption of analytes due to a very low concentration of metal ions. Synthetic fused silica contains less than 1 ppm of total metal ions while sodium (soft) glass and borosilicate (pyrex, kimax) glass contain metal cations at percent levels (Table 4). 148 The metal oxides present on the surface of sodium and borosilicate glass act as Lewis acid sites since a positive charge is centered on the metal while the negative charge is dispersed through the incomplete tetrahedral structure. A Lewis acid site adsorbs analytes by interacting with their lone pair electrons and can also interact with aromatic and olefinic $\pi$ systems. The greatly decreased metal ion impurities of fused silica decreases the likelihood of metal ions being present on the surface, and results in a more inert surface. The $\mathrm{SiO}_{2}$ in bulk silica consists of a silicon atom tetrahedrally bound to four oxygen atoms. The tetrahedron may share none, or up to four of the oxygen atoms with neighboring tetrahedra. Crosslinks formed by coordination through the oxygen atoms contributes to a high $\left(1800^{\circ} \mathrm{C}-2200^{\circ} \mathrm{C}\right)$ melting point for fused silica (Figure 25).150

The high melting point of fused silica limits its manufacture to commercial facilities due to the cost of high temperature drawing equipment and the high purity atmospheres needed. The manufacture of 


$$
a_{N}^{0}
$$

1
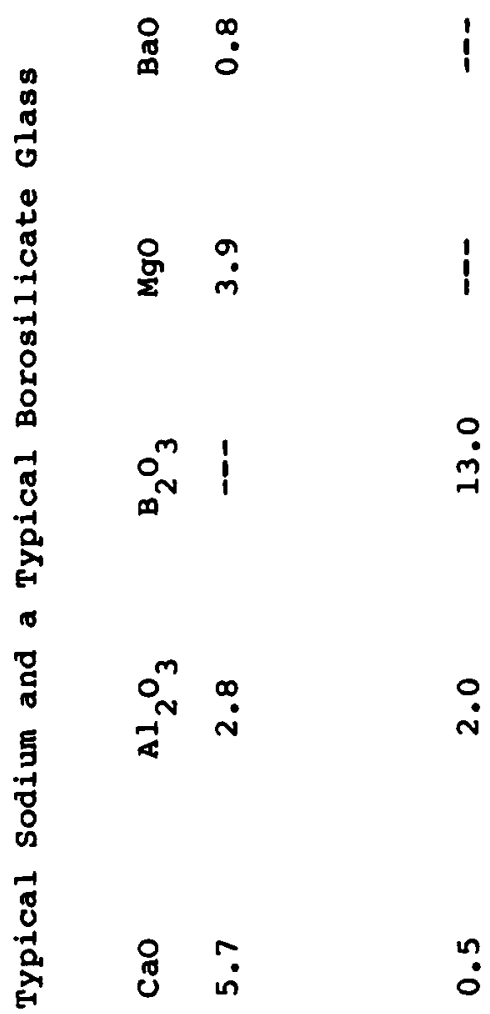

山
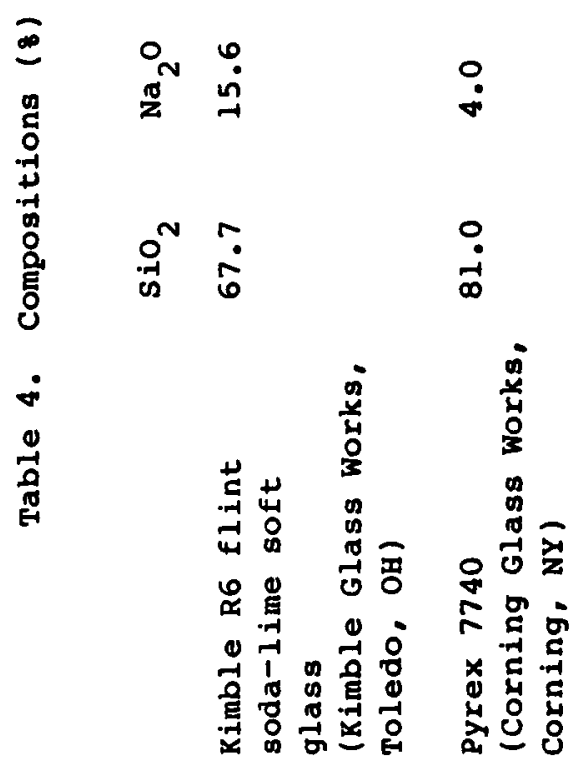


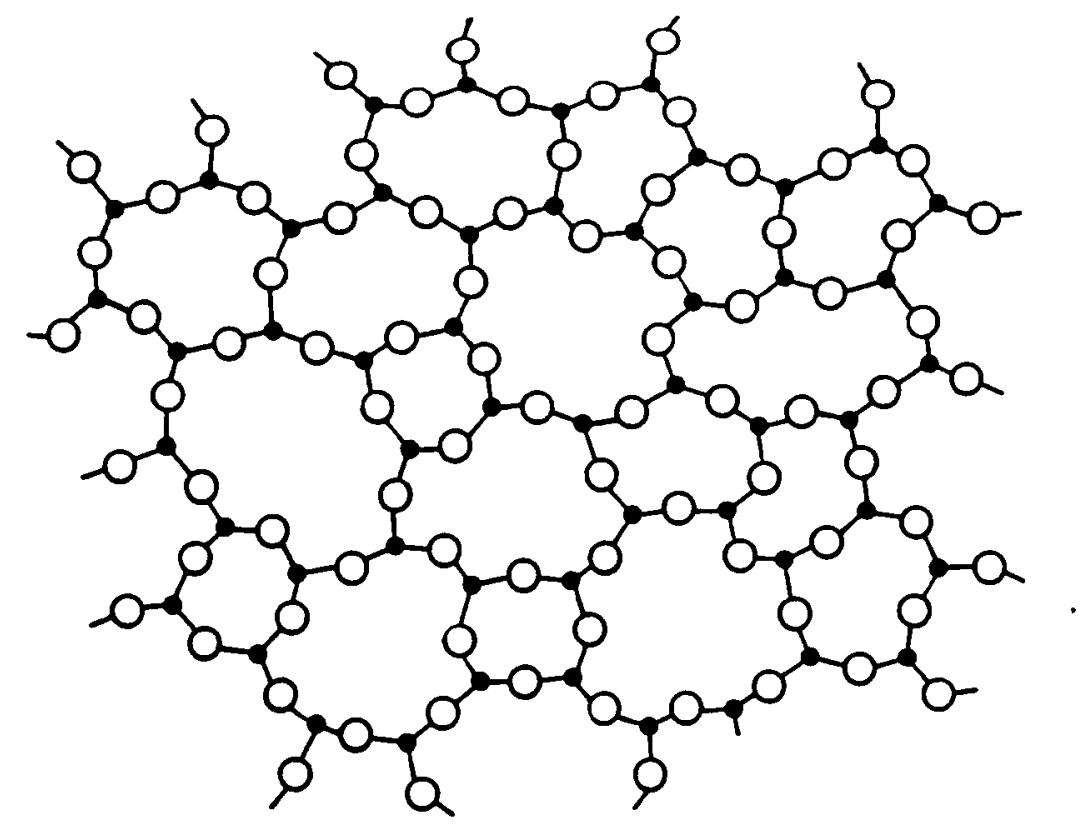

Figure 25. Structure of fused silica. 
fused silica capillaries is mostly limited to companies manufacturing optical fibers. The manufacture of fused silica starts with the flame hydrolysis of purified $\mathrm{SiCl}_{4}$ which reacts with water in the flame to produce $\mathrm{SiO}_{2} \cdot 151$

$$
\mathrm{SiCl}_{4}+2 \mathrm{H}_{2} \mathrm{O} \rightarrow \mathrm{SiO}_{2}+4 \mathrm{HCl}
$$

The $\mathrm{SiO}_{2}$ is built up on a substrate which is then removed to leave a hollow cylinder preform. The preform is thick walled and the dimensions of the preform will determine the ratio of the inside diameter to outside diameter of the final product. The preforms contain small scratches and other imperfections on both inner and outer surfaces. Since these imperfections could cause the resulting capillary to be very fragile, they are removed by first chemically treating with a rinse of dilute hydrofluoric acid, followed by a rinse with extremely pure water to remove ionic species. ${ }^{148}$ The preforms are then annealed and fire polished at high temperatures.

The preforms are inserted into a drawing machine where a combination of feed rollers and draw rollers work to pull the heated preform to the required dimensions. To achieve around a $2000^{\circ} \mathrm{C}$ drawing temperature, a variety of heat sources have been used including resistance and induction electrical heaters ${ }^{152}$, gas burners ${ }^{153}$ and lasers. ${ }^{154}$ The dimensions of the capillary are maintained at a constant size by using an infrared laser shining through the capillary to control the drawing rollers by feedback through a microprocessor. ${ }^{155}$ By using this computer control, uniform diameters are obtained throughout the length of the 
capillary. The fused silica capillary is inherently straight and is coiled onto a roller as it cools. In order to aid in the coiling process and to improve the handling characteristics, a polymeric coating is placed on the column as it is drawn. Various materials have been used to coat the exterior of the column, but polyimides and polyamides are the most widely used due a thermal stability of around $350^{\circ} \mathrm{C}$. The coating is generally applied by passing the column through a cup of the desired material and allowing coating to take place. Vitreous carbon has been used as a coating by passing butane around the hot column ${ }^{156}$, and most recently an aluminum coating has been developed commercially. ${ }^{157}$ Both of these coatings were developed to increase the upper temperature limit of the column coating.

The protective coating of the outside of the column accomplishes several tasks. It increases the flexibility of the column and gives it better handling characteristics, but more importantly it protects the column from atmospheric corrosion, scratching, fingerprints and dust. A consequence of the high draw temperature is the charging of the hot bare silica. This charged surface attracts dust, which can cause later column breakage. To decrease this problem, "clean room" atmospheres are maintained during the drawing process.

Fused silica columns with thin walls have a tensile strength of ca. $1.38 \times 10^{5} \mathrm{kPa}$ for a $0.3 \mathrm{~mm}$ column on a $150 \mathrm{~mm}$ diameter cage. Slight surface imperfections due to handling. etc. cause a great $\left(10^{4}\right.$ ) reduction in the strength of the material. 158 
Surface Characteristics of Fused silica

As noted previously, the characteristics of fused silica are highly dependent on its purity. Several grades of fused silica are currently available. The greatest distinction in purity is a function of the source of fused silica. Natural quartz is fused at high temperature under vacuum to produce a fused quartz. The purity of this fused quartz is highly dependent on the purity of the rock crystal starting material and any contamination that occurs during the fusion process. The metallic impurities in fused quartz may vary from 10-100 ppm depending on the source of starting material and fusion process. Synthetic fused silica prepared from the flame hydrolysis of $\mathrm{SiCl}_{4}$ is much purer with about 1 ppm total metallic impurities (Table 5). 148

Although the purity of fused silica decreases the likelihood of metal catalyzed adsorption and decomposition during a chromatographic analysis, adsorption effects on fused silica are observed. The cause of the activity of fused silica is believed to be the presence of hydroxyl groups on the surface. These hydroxyl groups are bound to a silicon atom which is presumably then bound into the bulk material through tetrahedral linkages.

The hydroxyl groups in porous silica can be described in terms of their bonding and location. When hydroxyl groups are attached to adjacent silicon atoms they are called vicinal, while geminal is used to refer to two hydroxyl groups on the same silicon atom. ${ }^{159}$ Hydroxyl groups occurring in the bulk silica are referred to as intraglobular hydroxyls. Under normal atmospheric conditions, the surface of the silica is covered with water adsorbed onto the surface hydroxyl 


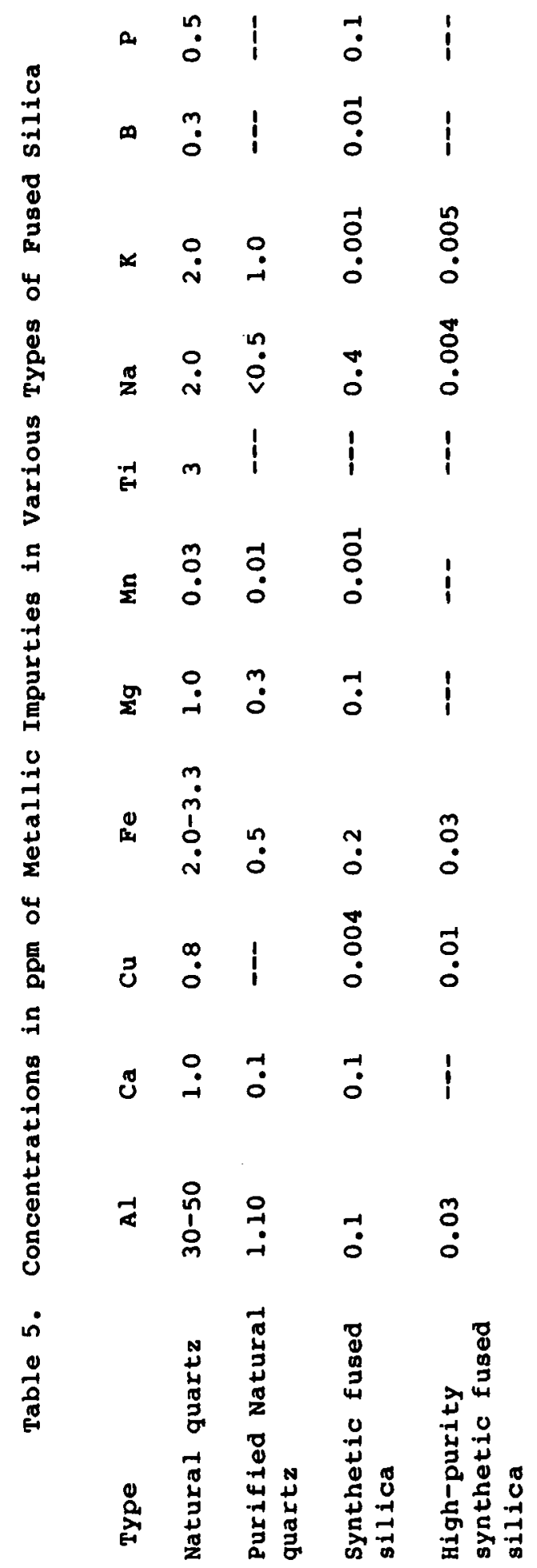


groups. 160 Heat treatment removes this adsorbed layer and uncovers the underlying hydroxyls. Further heating at high temperatures will dehydrate the surface. From room temperature to $165^{\circ} \mathrm{C}$, only physically adsorbed water is removed. From $160^{\circ} \mathrm{C}$ to $400^{\circ} \mathrm{C}$, hydroxyl groups are removed from the surface but the sites will rehydroxylate on cooling and exposure to water. Above $400^{\circ} \mathrm{C}$, a decreasing number of hydroxyl groups can be regenerated and above $800^{\circ} \mathrm{C}$ the rehydration of the surface does not occur on cooling and exposure to water (Figure 26).161,162

The concentration of surface hydroxyl groups on porous silica has been a subject of some interest, and the best estimate now seems to be a concentration of $4.6 \mathrm{oH} / \mathrm{nm}^{2}$ with each hydroxyl group bound to one silicon atom. 180 This value is for a porous silica surface which is annealed and fully hydroxylated.

The small surface area of glass and fused silica in a capillary column makes it very difficult for traditional surface techniques to be used. Almost all values for silica have been presented for porous materials. Recently, some results were obtained for glass and fused silica using tritium exchange of the surface hydroxyl groups. ${ }^{163}$ one interesting point of this study is the low concentration of hydroxyl groups on fused silica (Table 6). Due to the distances involved between adjacent hydroxyl groups, it is unlikely that intermolecular hydrogen bonding plays a large role in the surface chemistry.

The surface hydroxyl groups are acidic functionalities due to delectron cloud vacancies in the silicon atoms. 164 This gives fused silica surfaces a slightly acidic nature which is observed 


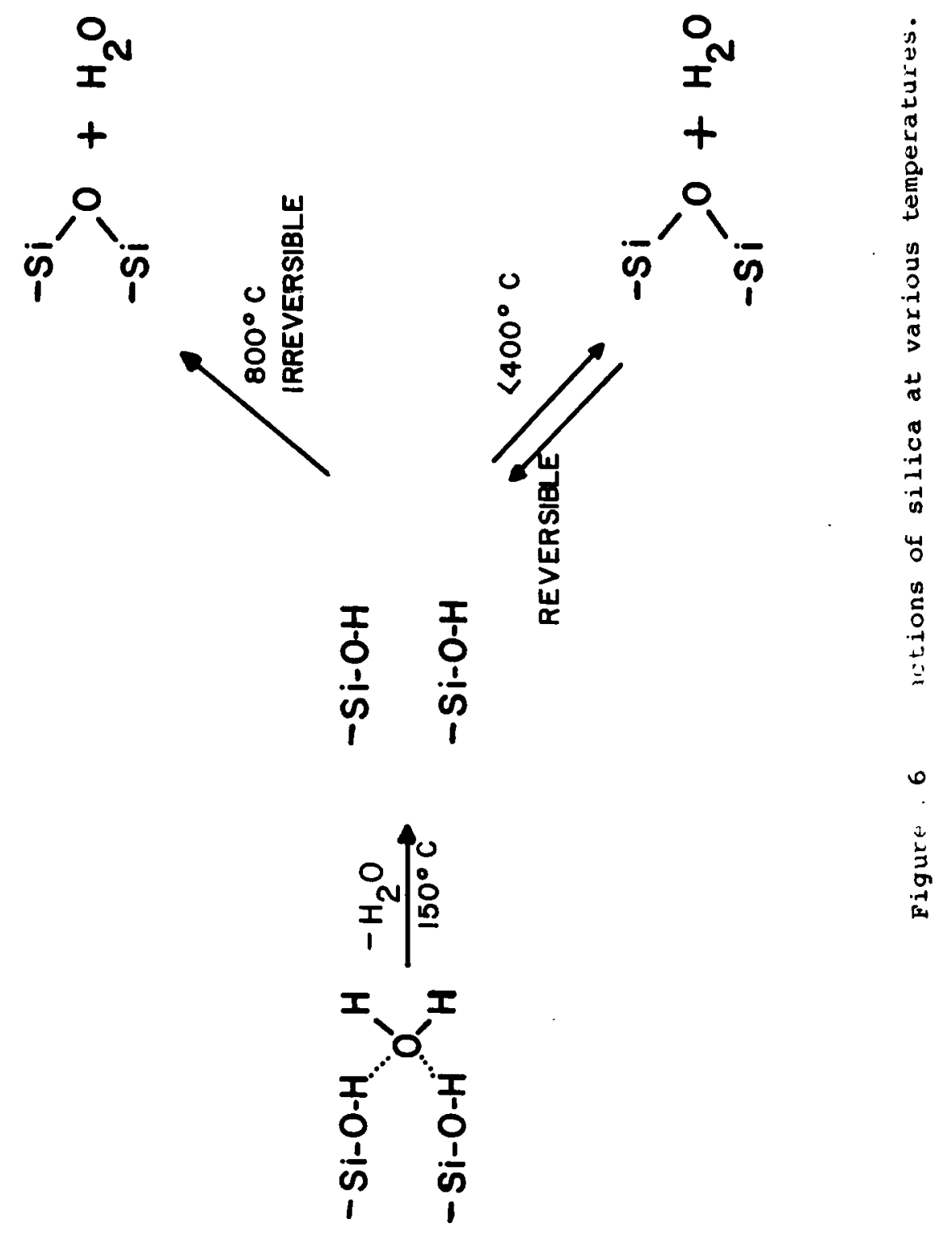


Table 6. Surface Hydroxyl Concentrations For Fused silica and Glass Capillary Columns as Determined by Tritium Exchange

\section{Type}

hydroxylated glass

dehydroxylated glass

fused silica

\section{Pretreatments}

acid leached, water rinsed, heat treated at $150^{\circ} \mathrm{C}$ for 12 hours

heat treated at $600^{\circ} \mathrm{C}$ for 8 hours

heat treated at $150^{\circ} \mathrm{C}$ for 4 hours
Surface hydroxy Concentration $\left(\mathrm{OH} / \mathrm{nm}^{2}\right)$

$2.84 \pm 0.09$

$0.36 \pm 0.02$

$0.21 \pm 0.04$ 
101

chromatographically by adsorption of amines and related compounds. The surface hydroxyl groups react strongly with water and allow several layers of water to be built up on the surface. 160 This adsorbed water layer can also act as an adsorption site, contributing to the surface activity in the same way as a surface hydroxyl.

Intense heating of silica $\left(700^{\circ} \mathrm{C}\right)$ can dehydrate the surface to form water and a strained siloxane bridge. ${ }^{165}$ These strained bridges are more reactive than surface hydroxyls due to a longer than usual bond length. 166 These structures probably do not influence fused silica greatly since above $900^{\circ} \mathrm{C}$ the structures decrease in activity presumably due to relief of bond strain. 165 The high draw temperature $\left(2200^{\circ} \mathrm{C}\right)$ of fused silica should relieve strained siloxane bridges.

\section{Capillary Column Surface Preparation}

The goal of capillary column pretreatment is to prepare the column for uniform coating by the stationary phase and at the same time deactivate any adsorbtive sites on the surface. The requirement that both tasks be accomplished leads to difficulties in column coatings. In order for a thin uniform film of polymer to be stable on the column wall, a proper match between the surface tension of the polymer and the surface energy of the column is necessary. For a stable film to coat the wall it is necessary for that film to wet the column wall.

When a liquid wets a solid, the liquid spreads so that the solidliquid and the liquid-gas interfaces increase in area while the solid gas interface decreases. The force per unit length in the liquid that opposes the spread of the liquid surface is $\gamma_{1}$ the liquid surface tension. 


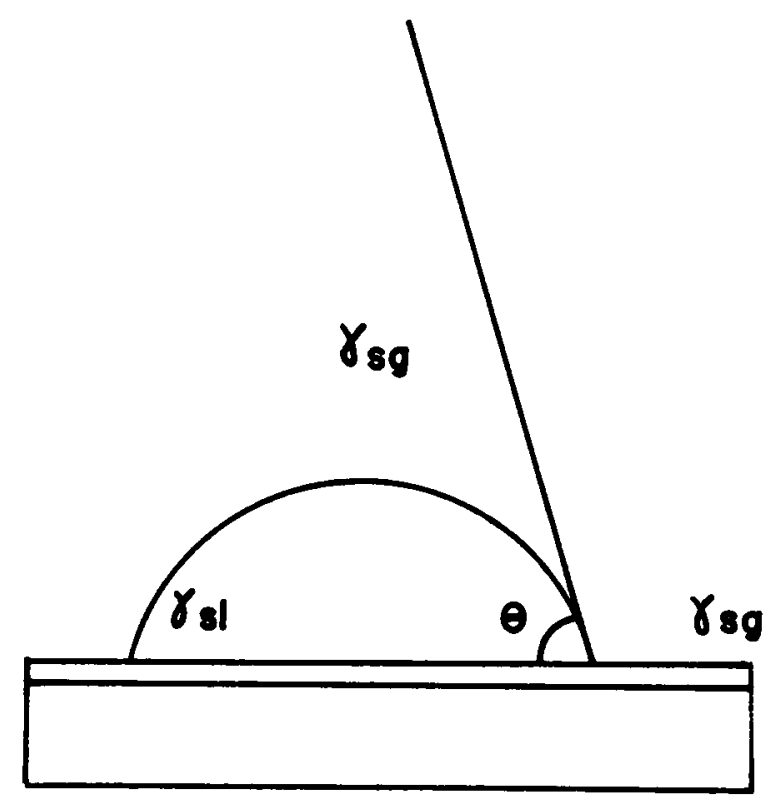

Figure 27. Illustration of the contact angle $(\theta)$ of a liquid drop on a solid surface. 
103

The contact angle, $\theta$, where the liquid-solid-gas interfaces all are in contact is defined as the angle through the liquid drop when a tangent to the drop and the surface is constructed (Figure 27). When a liquid wets the surface, the contact angle is zero. The contact angle increases as wetting decreases. Wetting describes the interaction between the liquid and the solid surface. When the liquid has more adhesive attraction for the solid than cohesive attraction for itself, wetting occurs and the liquid spreads over the surface.

When an equilibrium state has been established, the interactions between the solid, liquid and gas must balance (Figure 28). This equilibrium state can be represented by Young's Equation.

$$
\gamma_{s 1}-\gamma_{s g}+\gamma_{1 g} \cos \theta=0
$$

where $\gamma_{s g}$ is the surface energy of the solid in equilibrium with the gas (vapor of wetting liquid); $\gamma_{s g}$ is the surface tension of the liquid in contact with its vapor and $\gamma_{s l}$ in the surface energy of the solid in equilibrium with the liquid.

From this information, it can be seen that in order for a uniform film to wet the surface, the surface tension of the liquid must be less than that of the solid. Therefore, the surface of the solid must be suitable for the liquid being coated. Some values of surface tensions for various liquid phases are tabulated in rable 7 .

It has been reported that the surface energy of clean fused silica is quite high. ${ }^{168}$ However, extreme variability of the fused silica surface is seen in actual materials obtained from the manufacturer. ${ }^{169}$ 


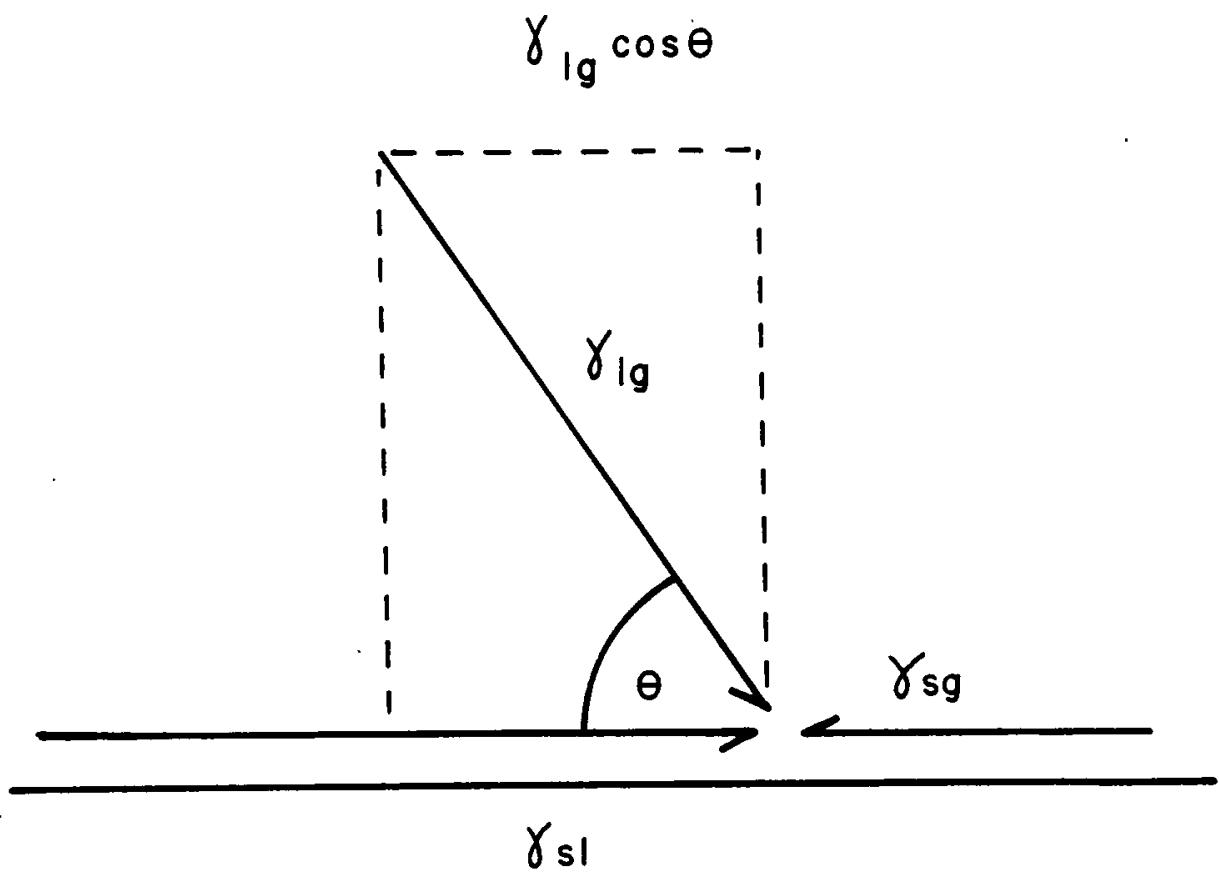

$$
\gamma_{s g}+\gamma_{l g} \cos \theta-\gamma_{s g}=0
$$

Figure 28. Graphical depiction of Young's Equation. 
Table 7. Surface Tensions of Various Stationary Phases with Their Polarities

\begin{tabular}{|c|c|c|c|}
\hline Stationary Phase & $\begin{array}{l}\text { Chemical } \\
\text { Type }\end{array}$ & $\begin{array}{l}\text { Burface tension } \\
\text { (dynes } / \mathrm{cm} \text { ) }\end{array}$ & $\begin{array}{l}\text { CPI } \\
\& \text { Polarity }\end{array}$ \\
\hline ov-101 & methyl polysiloxane & 20.4 & 5 \\
\hline ov -3 & $\begin{array}{l}108 \text { Phenyl methylpoly- } \\
\text { siloxane }\end{array}$ & 21.8 & 10 \\
\hline OV -210 & $\begin{array}{l}508 \text { Trifluoropropyl } \\
\text { methylpolysiloxane }\end{array}$ & 22.8 & 36 \\
\hline Squalane & $c_{30}$ alkane & 30.0 & 0 \\
\hline ov -17 & $\begin{array}{l}508 \text { Phenyl methylpoly- } \\
\text { siloxane }\end{array}$ & 31.4 & 21 \\
\hline Carobwax 400 & Polyethylene glycol & 44.2 & 61 \\
\hline Tris & $\begin{array}{l}1,2,3-\text { Tris (cyanoethoxy) } \\
\text { propane }\end{array}$ & 49.2 & 98 \\
\hline
\end{tabular}


These variations can be caused by absorption of materials on the active surface either during drawing or subsequent storage. Once the surface has been contaminated, it is not easily renewed.

The high surface energy of the silica therefore causes two problems. If the surface is clean, the stationary phase will coat efficiently, but active silanols on the surface will cause absorption of polar analytes. If the surface has been exposed to contaminants before coating, the surface energy will vary greatly and the stationary phase may or may not wet the surface. Therefore, column pretreatment must cover active sites on the surface while maintaining a surface energy that is high enough for efficient wetting by the stationary phase.

One method of increasing the wettability of the surface would be to roughen the surface. This roughening would increase the surface area and stabilize the polymer film. Unfortunately while surface roughening has been very useful in glass capillary preparation, fused silica columns have not been as amenable to this treatment. Roughening of fused silica has been shown to greatly decrease the tensile strength of the material and increase the fragility. ${ }^{170}$ The use of HF to promote the growth of whiskers on glass columns has been very useful in stabilizing polar phases. The whiskers increase the surface area and the amount of liquid phase which will form a stable film. Droplet formation of the phase on the whiskered surface is rarely observed, but the whiskers are very active sites for sample absorption. ${ }^{171,172}$ The use of this technique with fused silica capillaries results in holes in the surface rather than whisker growth. ${ }^{173}$ 
107

Another alternative for surface roughening would be the deposition of a layer of small particles on the column surface. This technique has been successfully applied to glass ${ }^{174-177}$ but on fused silica, these particles would result in a loss of inertness.

The best methods for increasing the wettability of the fused silica surface are obtained by chemical rather than physical means. A variety of techniques have been used in GC to modify the surface chemically. The first fused silica columns prepared by Dandenam and zerenner were deactivated with pyrolyzed Carbowax. ${ }^{\text {TM,178 }}$ This deactivation layer serves two purposes. First, it reacts with surface silanols to render them non-adsorptive and second, the pyrolyzed carbowax provides a coating with a surface energy of 45 dynes/cm which is high enough to coat a variety of semi-polar and non-polar phases.

The mechanism of Carbowax pyrolysis is not fully known. Carbowax is a polyethylene glycol which is available in a range of molecular weights. Some recent attempts to produce polyethylene glycols with narrower molecular weight distributions and/or higher molecular weights have been marketed under the name superox. The goal of this approach has been to form a si-o-c bond with the surface silanol and effectively deactivate the silanol. The first reports of a polyethylene glycol deactivation using Carbowax $20 \mathrm{M}(20,000$ M.W.) was by Aue et al. on a diatomaceous earth support. ${ }^{181}$ The procedure involved heating at $280^{\circ} \mathrm{C}$ and then extracting with hot methanol. This procedure was thought to produce a monolayer coverage of long polyethylene glycol chains on the surface. The first application to glass capillary columns was by Cronin 
who dynamically coated a 28 solution of Carbowax 20M in methylene chloride, sealed the column ends and heated the column at $280^{\circ} \mathrm{C}$ for 16 hours. ${ }^{182}$ After rinsing the columns with methylene chloride and methanol, a well-deactivated layer was formed. Many variations of this procedure were subsequently reported. 183,184

The formation of the $\mathrm{Si}-\mathrm{O}-\mathrm{C}$ bond can also be produced by chlorinating the silanol and then reacting with an alcohol. However, the si-o-c bond has a low thermostability and a susceptibility for hydrolysis which has greatly limited its application to capillary GC. ${ }^{185}$ In an attempt to increase the thermal stability of the polyethylene glycols when used as stationary phases, the narrower molecular weight range superox polymers were developed. This has led to some increase in stationary phase thermal stability but has not greatly decreased the thermal bleed caused by the deactiviation layer. 186,187

One reason for this finding may be the mechanism for Carbowax pyrolysis proposed by verzele. On silica surfaces at high temperature, the polyethylene glycol is thought to decompose to species like oxirane which then chemically bond to the surface hydroxyl groups (Figure 29). 188 In this mechanism, an increased molecular weight of polymer would have little effect, since it is a decomposition product which actually bonds to the surface. The small increases in thermal stability of the deactivation layer may be due to more uniform coating and resistance to droplet formation by the more viscous, higher molecular weight polyglycols. 


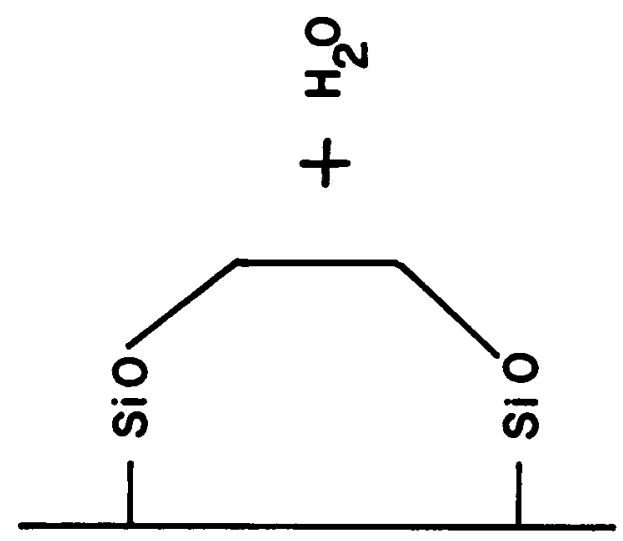

$n$
$0-1$
0
2
0
0
2
2
2

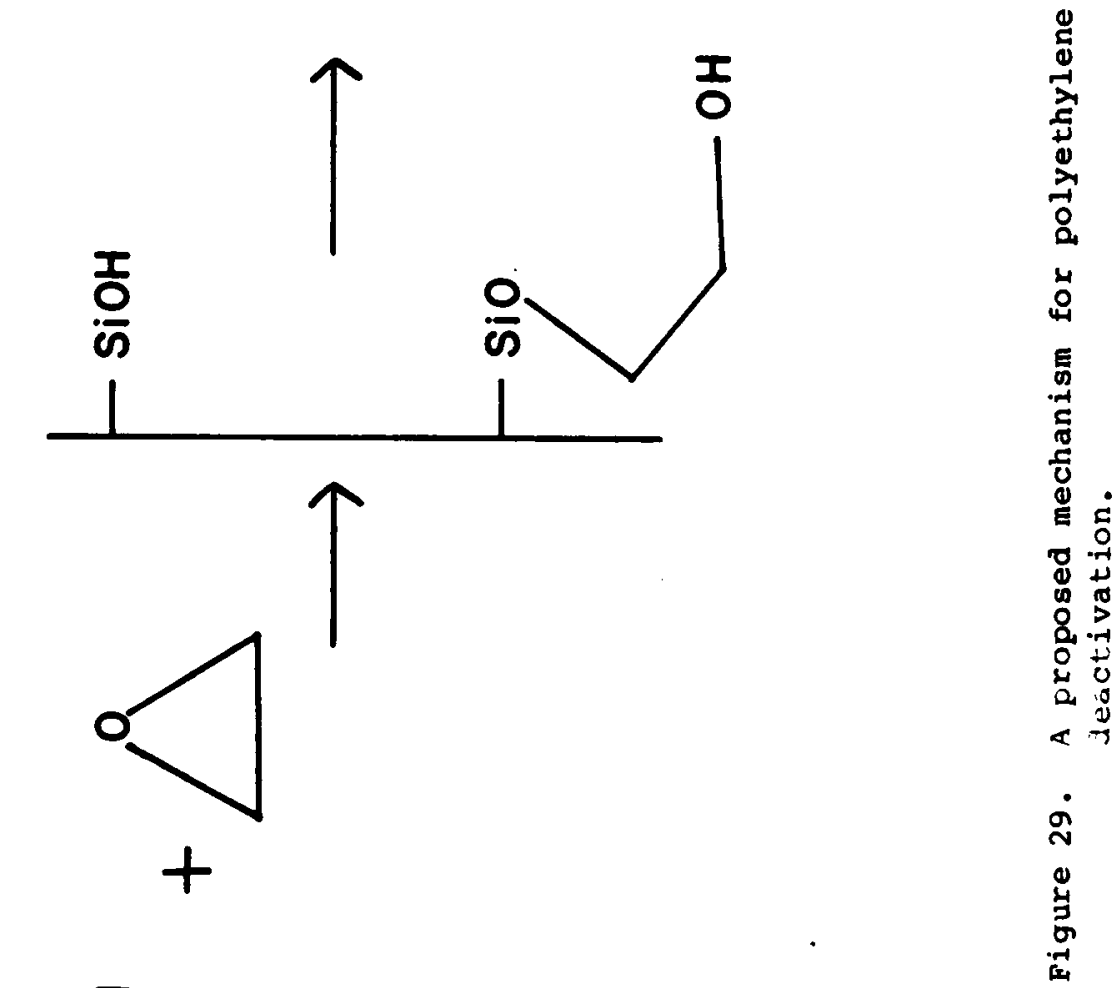

-1
0
वे
0

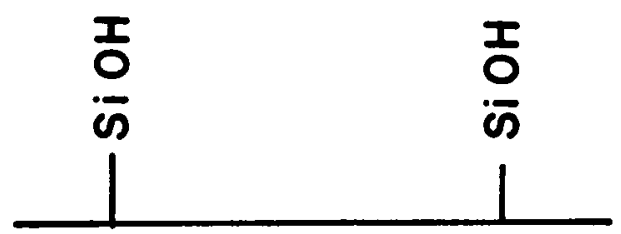


110

Because of the instability of the $\mathrm{si}-\mathrm{O}-\mathrm{C}$ bond and the observation that a polar deactiviation layer beneath a non-polar phase can cause the selectivity of the column to be affected ${ }^{148}$, the use of polyethylene glycols for the deactivation of capillary GC columns has all but ceased. This is unfortunate since Carbowax deactivation is effective in decreasing surface adsorption while at the same time maintaining a high surface energy.

The most commonly used approach for capillary GC deactivation involves silylation reactions which form the very stable si-0-si linkage through the surface silanol groups. A variety of reagents have been used to convert silanols on the surface to silyl ethers.

The most common reagents for silylation or silanization are trimethylchlorosilane, hexamethyl disilazane or mixtures of the two. 189,190 The resulting deactivation layer is a low energy, methylated surface. Similar results were obtained by pyrolyzing a methylsilicone polymer $(\mathrm{OV}-101)$ in the column at $450^{\circ} \mathrm{C} .191$ However, the application of this technique to more polar stationary phases (OV-17) was found to be inappropriate. 192

Very promising results have been obtained using cyclic siloxanes as deactivation reagents. These compounds undergo a ring opening reaction at high temperatures to bond to surface silanol groups (Figure 30 ). 193 Work has been performed using octamethyl cyclotetrasiloxane $\left(D_{4}\right)$ for non polar columns ${ }^{194}$ and other cyclic siloxanes containing trifluoropropyl and cyanopropyl functionalities for more polar stationary phases. ${ }^{195}$ One advantage of the cyclic procedure is the avoidance of production of 


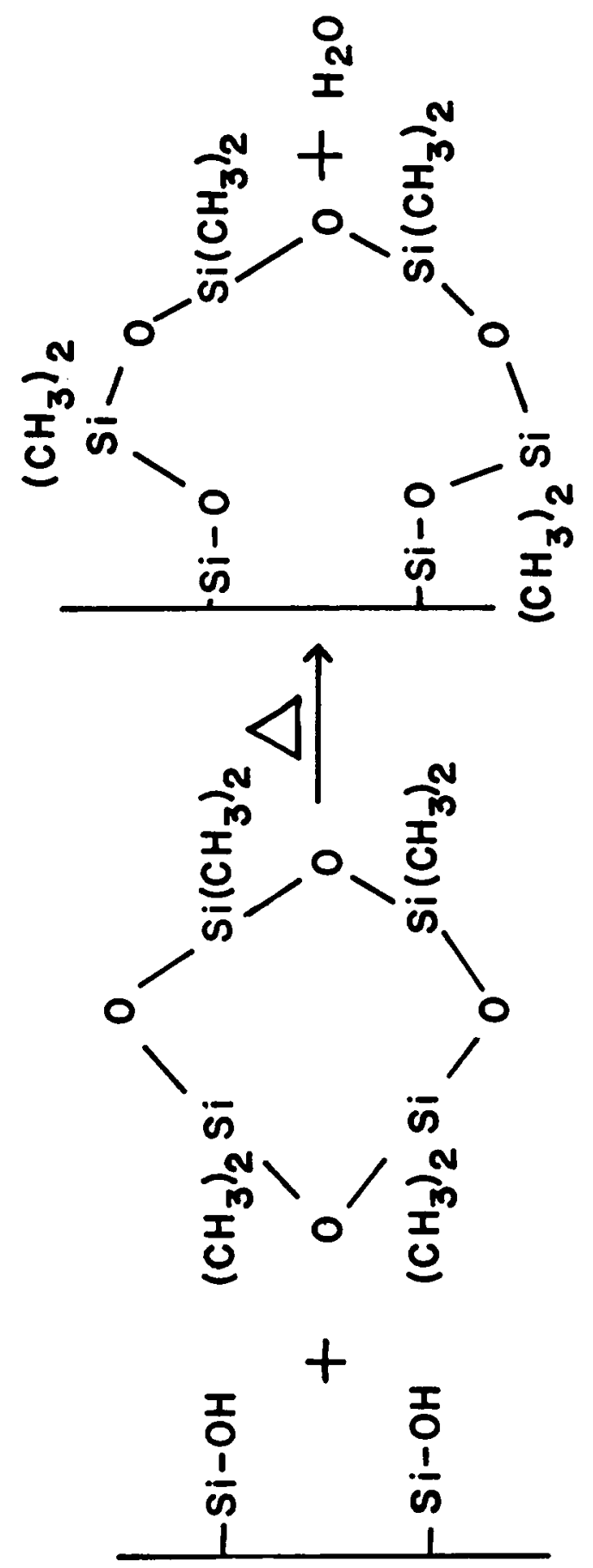

Ȯ 
112

$\mathrm{HCl}$ or $\mathrm{NH}_{3}$ gas during the process which could later cause column deterioration.

\section{Surface Evaluation}

Evaluation of the surface energy of the capillary column after a deactivation treatment is important due to the influence that surface energy will have on later wettability by the stationary phase. Measurement of the column surface energy can be accomplished by nonchromatographic methods using capillary rise techniques. ${ }^{196}$ since the column is directly used in the determination, capillary rise measurements provide information on the actual system. This prevents the errors that would arise from trying to apply data from a model system to the actual system. For a straight capillary column such as a fused silica column immersed in a liquid, the upward force exerted on the liquid is given by $2 \pi r \gamma_{1} \cos \theta$ where $r$ is the radius of the column, $\gamma_{1}$ is the surface tension of the 1 iquid and $\theta$ is the contact angle. The downward force opposing the rise is given by $\pi r^{2}$ hpg where $h$ is the height of the liquid and $g$ is the acceleration due to gravity. At equilibrium:

$$
2 \pi r r_{1} \cos \theta=\pi r^{2} h \rho g
$$

which by rearrangement, allows the contact angle $\theta$ to be determined.

$$
\cos \theta=\frac{\text { hpgr }}{2 \gamma 1}
$$


Table 8. Surface Tensions ( $\gamma l$ ) and Densities $(p)$ for various Mixtures of Methanol/ $\mathrm{H}_{2} \mathrm{O}$ used to Determine Critical Surface Energies by Capillary Rise.

Mixture (MeOH/ ${ }_{2}$ 을

$100 / 0$

$90 / 10$

$75 / 25$

$70 / 30$

$60 / 40$

$50 / 50$

$25 / 75$

$20 / 80$

$10 / 90$

$0 / 100$ y1 (dynes/cm)

22.1

24.9

28.3

29.7

32.6

34.9

45.8

51.6

58.5

72.0 $p(g / m l)$

0.792

0.820

0.859

0.872

0.894

0.916

0.962

0.967

0.982

1.000 
114

Zisman proposed that a series of liquids of varying $\gamma_{1}$ could be used to characterize the surface energy of a solid such as the interior of the capillary. A plot of $\cos \theta$ vs. $\gamma_{1}(z$ isman plot) could be constructed which would exclude values of $\cos \theta=1$ (complete wetting). The remaining points, which would come from liguids which incompletely wet the surface could be extrapolated to $\cos \theta=1$ and the corresponding surface tension would give the critical surface energy of the solid. Several applications of this technique to chromatography have been reported. 197 The most frequently used liquids are mixtures of methanol/water which cover a wide range of surface tensions and are readily available (Table 8 ). 198 The advantage of this technique is the ease with which an evaluation of column surface energy can be made without the need to coat quantities of columns by the method of trial and error.

\section{Capillary Column Coating}

The objective of column coating is to deposit the stationary phase in a thin uniform coating over the length of the column. The problem of coating a thin $(0.1-8 \mu \mathrm{m})$, uniform film on the inside of a tube which has an inner diameter which is less than $0.75 \mathrm{~mm}$ for capillary GC and less than $0.1 \mathrm{~mm}$ for capillary SFC is not trivial. Many methods have been developed to coat small diameter columns. These methods can be classified into two main divisions, dynamic coating techniques and static coating techniques. 


\section{Dynamic Coating Techniques}

Dynamic coating involves partially filling a column with a concentrated solution of stationary phase. The plug is pushed through the column by $\mathrm{N}_{2}$ gas pressure and the polymer is deposited on the walls as a thin film. The $\mathrm{N}_{2}$ flow is continued until all of the residual coating solvent is evaporated. The advantage of the dynamic coating method is that it is quickly and easily accomplished. The disadvantage is that due to a number of factors, the film deposited may vary quite significantly in thickness throughout the column. This makes the dynamic coating method unsuitable for coating of efficient capillaries, but suitable for some methods of column deactivation where deposited film thickness is not critical. Non-uniformity of the film thickness can be attributed to several factors. The deposition of the film is dependent on the linear velocity of the plug. As the plug moves through the column, the column pressure drop decreases as the plug moves toward the end of the column. An optimum coating velocity of $1-2 \mathrm{~cm} / \mathrm{min}$ may increase to several times that value as the column end approaches. To minimize this effect a length of empty tubing is usually attached at the end of the column to serve as a buffer column and minimize the pressure drop across the column to be coated.

Another difficulty arises from the change in coating solution concentration during the coating process. As the polymer is deposited on the wall of the column, the concentration of polymer in the plug is decreased. As the concentration decreases, the viscosity of the solution decreases, which leads to the plug velocity increasing as coating proceeds. 
One problem occurs as a function of the orientation of the column. In some cases, the polymer deposited on the column wall may have a low enough viscosity to flow to lower parts of the column. 199 To prevent the stationary phase from collecting in the bottom of each column coil, the column is usually placed horizontally so the coils will lie flat. Although dynamic coating does not usually result in a uniform film of stationary phase, it is a rapid and easy method for rinsing the column with solvents for clean-up or depositing reagents for column activation and deactivation. These procedures do not require uniform film deposition and so are unaffected by the drawbacks of this technique.

\section{Static Coating Techniques}

In 1968, a comparison of static and dynamic coating by Bouche and Verzele 200 showed the weaknesses of the dynamic coating procedure and promoted the use of the static technique. Although the first capillary columns were made using a static procedure, static coating was not widely use or easily performed. In the first work by Golay, the filled capillary was slowly pushed into an oven where the solvent evaporated. The meniscus formed by the evaporating solvent slowly moved backwards as the solvent was removed. In practice, smooth coating did not usually result and the meniscus moved back and forth as a result of uneven heat application.

The static coating technique is distinguished by three characteristics. The first characteristic is that the amount of phase in the column can be easily determined. The stationary phase is 
117

dissolved in a volatile solvent and the amount of phase in the column can be calculated by the concentration of the polymer solution and the volume of the column. The second characteristic is that the stationary phase does not move axially through the column. It is deposited in situ as the solvent is evaporated usually under vacuum. This results in the term "static" being applied to the coating process. Finally, the polymer is deposited as a final coating. The solvent is almost completely removed as the meniscus retreats, so viscous flow of a partially solvated phase does not result.

\section{Column Crosslinking}

Crosslinking of the capillary column involves the formation of links between the linear chains of the polymeric stationary phases. Crosslinking increases the molecular weight of the polymer and decreases the effect of temperature on phase viscosity. These factors allow crosslinked phases to be used over a wider temperature range. For SFC, the most important property of crosslinking is that when the polymer's molecular weight is increased by the formation of a network of crosslinks, the polymer becomes insoluble. This property is of particular importance in SFC since the supercritical fluid is a good solvent and can dissolve the stationary phase. Early work in column crosslinking concentrated on the formation of si-o-si crosslinks to the polymer backbone. However, problems with increased column activity due to residual silanol groups and lowered column efficiencies due to decreased gaseous diffusion in highly cross-linked (10-508) systems have made formation of Si-C-C-Si crosslinks preferable. 208 


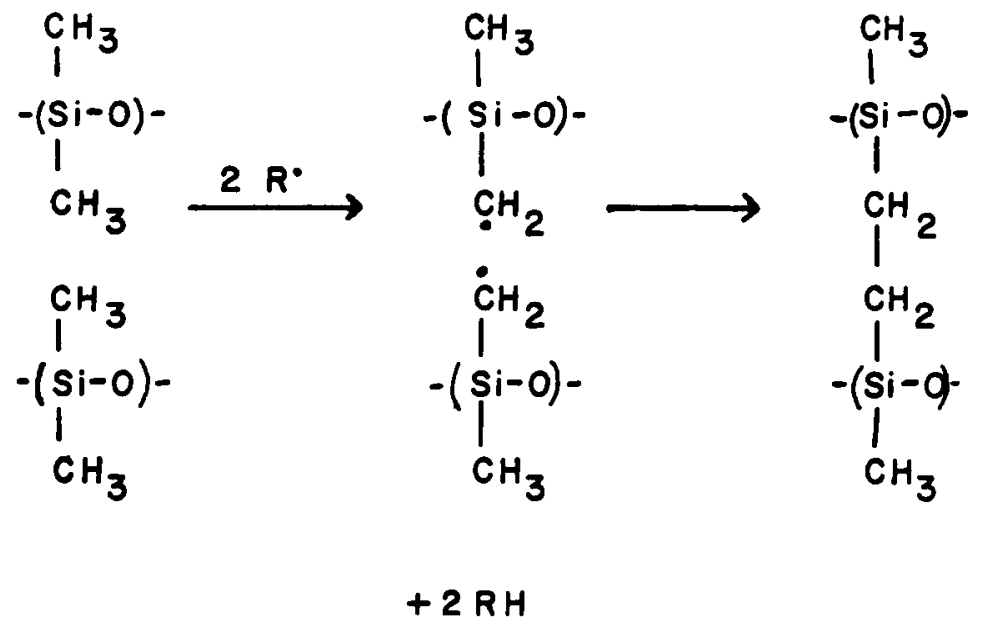

Figure 31. Free radical mechanism of crosslinking. 
The preferred method of column crosslinking is a free radical initiation of $\mathrm{si}-\mathrm{C}-\mathrm{C}-\mathrm{Si}$ bond formation which requires a chemical free radical initiator or a source of ionizing gamma radiation ${ }^{209}$ (Figure 31). For most laboratories, a ${ }^{60} \mathrm{Co}$ source is not readily obtainable and work with chemical initiators is more common. The first use of peroxides for stationary phase crosslinking was very encouraging but subsequent investigations have shown that some peroxides such as benzoyl peroxide and 2,4-dichlorobenzoyl peroxide can generate decomposition products such as benzoic acid and 2,4-dichlorobenzoic acid which catalyze the breakdown of the stationary phase. 210 Dicumyl peroxide (DCP) is the most common free radical initiator for capillary GC columns since its decomposition products seem to be less harmful to the stationary phase.

Recently, the introduction of various azo compounds have provided another alternative to peroxide use. 210,211 several azo compounds have been reported to serve as successful crosslinking initiators. The most popular is azo-tert-butane (ATB). This compound has the advantage of existing as a liquid with a fairly high vapor pressure. Unlike DCP which must be added as a solid in solution to the coating solvent, ATB can be added after column coating and conditioning by saturating the column with ATB vapors. The use of ATB is popular since ATB decomposes to $\mathrm{N}_{2}$ and butane or butene. These are thought not to detrimentally affect phase polarity if they should be incorporated into the stationary phase. 
120

Initiation of the crosslinking reaction occurs after the initiator has been added, either by incorporation in the coating solvent or by later vapor saturation. This is generally accomplished by sealing the column ends and holding at a temperature which will decompose the initiator. Various decomposition temperatures are given in Table 9.210 This is the necessary temperature for decomposition of $1 / 2$ the initiator in a 15 minute period.

In crosslinking of the phase, polarity plays an important role. The majority of free radical reactions involves hydrogen atom extraction from the methyl group. When high levels of phenyl or cyanopropyl functionalities are present, crosslinking efficiency is greatly reduced. For more polar phases, vinyl or tolyl group incorporation in the phase has been shown to increase crosslinking efficiency. 212,123

\section{Capillary Column Evaluation}

There are two distinct methods of testing a capillary column. The first method involves a specific test and the second involves a general test. A specific test is usually undertaken to demonstrate the suitability of the column for a particular application. If a column is to be used exclusively for a particular analysis, for example, to resolve two isomers, then a test mixture containing the isomers at the expected concentration in the sample matrix will provide the necessary information. The number of specific test mixtures is as large as the number of samples amenable to analysis.

For evaluation of the actual chemistry and column processes occurring, it is better to have a general test mix which can be used to 
121

\section{Table 9. Temperatures Needed to Decompose One-Half of the Compound in 15 Minutes}

\section{Eree Radical Initiators}

2,4-dichlorobenzoyl peroxide (DCBP)

dibenzoyl peroxide (DBP)

di-t-butyl peroxide (TBP)

dicumyl peroxide (DCP)

azo-t-butane (ATB)
Activation Energy

(kcal/mol)
Decomposition

Temperature $\left({ }^{\circ} \mathrm{C}\right)$
28.1

29.9

37.5

38.0

43.0
87

109

160

142

187 
describe the column in terms of several parameters. Some of the parameters which should be evaluated include efficiency, active adsorption sites, acidity or basicity of the column surface, and stationary phase film thickness.

The best and most widely accepted test mixture is one proposed by Grob. 214,215 This test is standardized so that no independent optimization of temperature or flow conditions is required. The mix contains both active polar compounds and inactive non-polar alkanes and methyl esters. This allows column activity to be related to individual effects on various components and allows quantitative aspects of adsorption to be measured by construction of an expected elution height of a polar peak by comparison to the curve formed by the unadsorbed nonpolar peaks (Figure 32 ).

Two test mixes are available, one for polar columns and the second for non-polar and semipolar columns. The components with their abbreviations and concentrations are given in Table 10. The phenol and the aniline allow determination of strongly acidic and basic sites while the carboxylic acid and amine show weaker acid/base interactions. The hydroxyl groups on the octanol and the diol provide a very sensitive test for hydrogen bonding sites on the surface including surface silanols. One added benefit has been the determination by Grob of elution temperatures for methyl dodeconate on various stationary phases. By observing the elution temperature on the column of interest for this component, and extrapolation from the known value, stationary phase film thickness can be determined experimentally. Due to the low thermal 


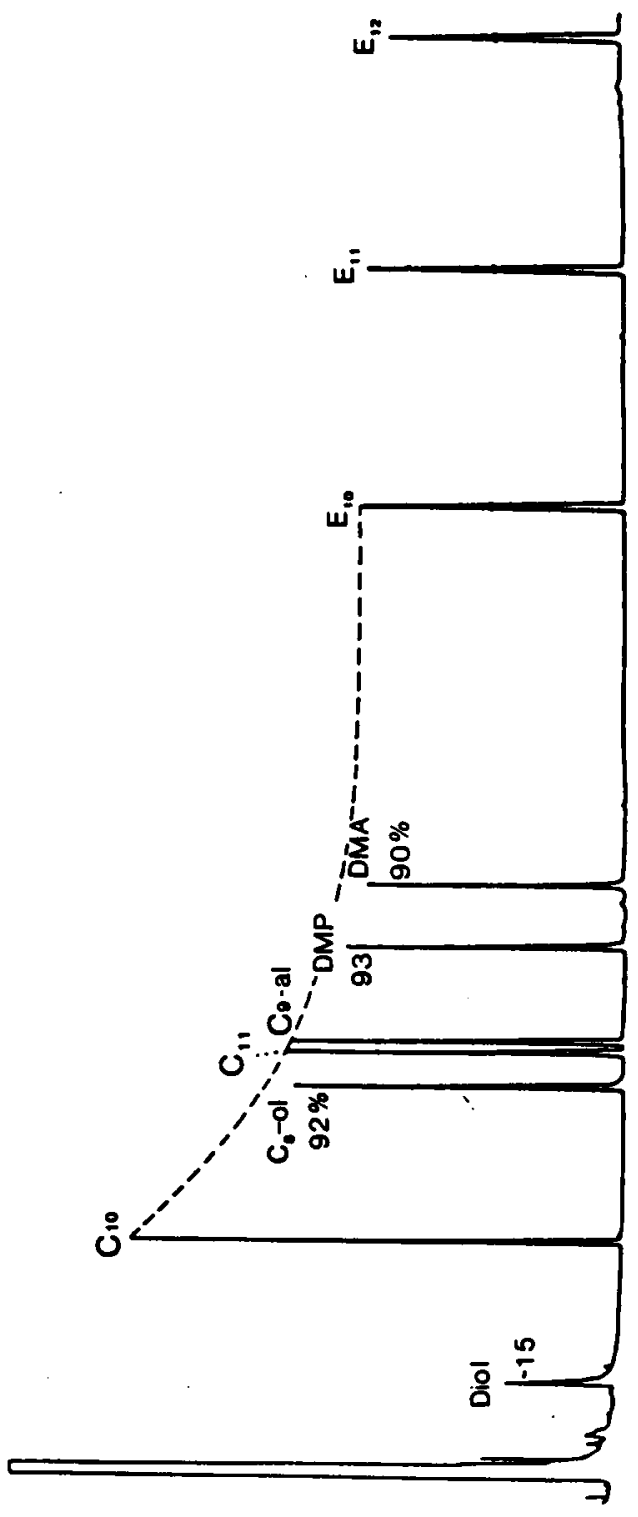

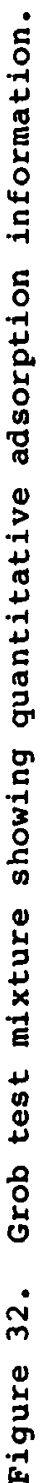


Table 10. Concentration of the Grob Test Mixture II

\section{$\underline{1}$}

1

2

3

4

5

6

7

8

9

10

11

\section{Component}

decane $(c-10)$

dodecane $(c-12)$

1-octanol (ol)

2,3 butanediol (D)

2,6 dimethyl aniline (A)

2,6 dimethylphenol (P)

2-ethylhexanoic acid (S)

dicyclohexylamine (Am)

methyl dodecanoate $\left(\mathrm{E}_{10}\right)$

methyl undecanoate $\left(E_{11}\right)$

methyl dodecanoate $\left(E_{12}\right)$

\section{Concentration $\mathrm{ng} / \mathrm{\mu l}$}

47.8

48.9

61.7

105.6

56.9

53.9

67.2

56.7

67.2

65.6

63.9 
stability of some phases, an initial temperature of $35^{\circ} \mathrm{C}$ is used for the analysis. The temperature programming rate is varied with column length so that the sample elution temperature is independent of column length when operated at a standard linear velocity. For further experimental details, the original work by Grob is recommended. 214,215 
CHAPTER V

EXPERIMENTAL

\section{Introduction}

Four major concerns in SFC column technology were addressed in this study. The first point of interest was the stability of the deactivation layer which underlies the stationary phase. The second concern was the ability of crosslinking methods to effectively immobilize the phase and prevent dissolution by the supercritical solvent. The third was the development of suitable instrumentation for SFC so effects of this work could be studied.

The evaluation of these first two concerns is best addressed by gas chromatographic methods. Gas chromatography provides a sensitive method of measuring the interaction between a sample probe and the analytical column. The columns used in SFC are similar chemically to GC columns and differ only in internal diameter. By using the larger GC columns, more information can be obtained by using techniques like the Grob text mix to monitor column activity and film thickness.

The third concern is the quantiative reproducibility of SFC. Once well deactivated, crosslinked columns are developed, their effect on the reproducibility of the technique needs characterizing. In order to do this, SFC with various samples types must be performed.

\section{Materials and Reagents}

All fused silica tubing was obtained from Polymicro Technologies, Inc. (Phoenix, Az) except for the $50 \mu \mathrm{m}$ i.d. which was obtained from 
Hewlett-Packard Co. (Avondale, PA). All solvents used were "Baker ResiAnalyzed" grade or for the nitric acid, "Baker Instra-Analyzed" grade (J. T. Baker, Phillipsburg, NJ). Column deactivation reagents included Carbowax 20M from Foxboro/Analabs (North Haven, CT), Superox 20M from Alltech/Applied Science (Deerfield, IL), octamethylcyclotetrasiloxane $\left(D_{4}\right)$, octaphenylcyclotetrasiloxane $\left(\mathrm{Ph}_{4}\right)$, and tetrakis ( $B$-cyanoethyl) tetramethylcyclotetrasiloxane from Petrarch Systems (Bristol, PA). The crosslinking initiators azo-tert-butane (ATB) and dicumyl peroxide (DCP) were obtained from Fairfield Chemical Co. (Blythewood, SC) and Pfaltz and Bauer, Inc. (Waterbury, CT) respectively. The comprehensive Grob test mixture II was obtained from Fluka Chemical Corp (Happauge, NY) while the alkane, alcohol and acid standards were obtained from Sigma Chemical Co. (St. Louis, MO). The stationary phase ov-1701 was obtained from American Scientific Products (Columbia, MD).

\section{Experimental Aspects of Column Coating}

The most convenient, and the currently accepted method of static coating involves maintaining the column at a very constant temperature. The column is then filled with the coating solution and allowed to thermally equilibrate. One end is carefully sealed to provide a leak tight closure with no trapped air, while the other column end is attached to a vacuum source. An ionization gauge monitors the pressure of the system and indicates when coating is completed. A schematic of this system is shown in Figure 33. 


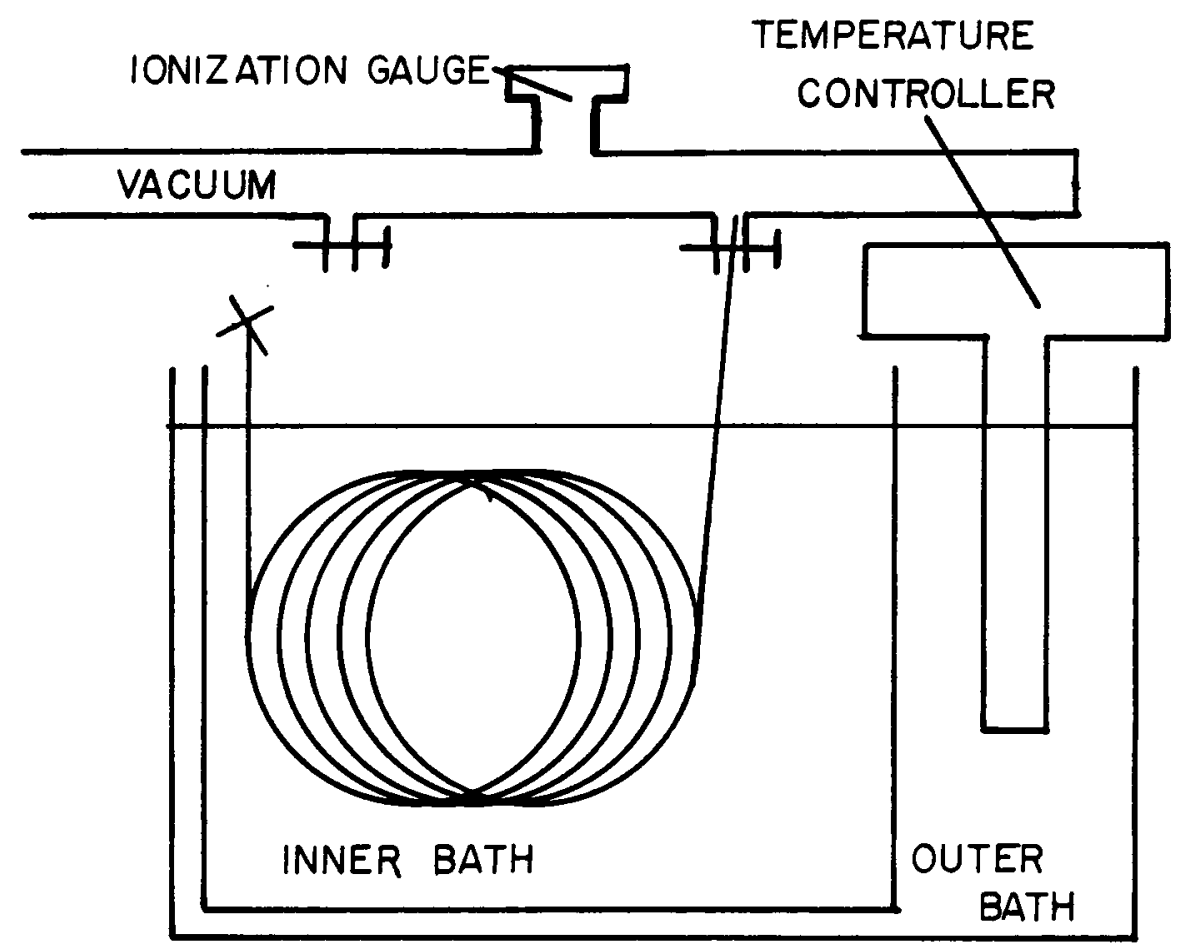

Figure 33. Capillary coating apparatus. 
The importance of thermostating the column is much greater for the small diameter SFC columns than for the larger diameter GC columns due to longer coating times. A thin tube filled with liquid behaves like a thermometer. The response to a change in temperature is a change in the volume of the coating solution. Table 11 shows the distance for various column lengths that the meniscus would travel in a column as the result of a change in temperature. To prevent the expansion and contraction of the coating solution, which would result in uneven deposition of liquid phase, a double insulated water bath was uṣed with a circulating water heater (Bioblock Scientific Model \#86602, Paris, France) to stabilize the temperature. Temperatures were maintained at $30^{\circ} \mathrm{C}$ throughout the coating process. The columns were usually allowed to remain under vacuum overnight to be certain that all solvent was removed. A typical 10 meter, 100 um i.d. column could be coated in 4 hours using pentane as the coating solvent while dichloromethane solutions at $30^{\circ} \mathrm{C}$ required about 6 hours to coat.

\section{Coating solutions}

The coating solution should be able to dissolve the polymer in all proportions so that smooth deposition occurs. In addition, the resulting solution should be of low viscosity so that extreme pressures are not required to push the solution through the columns. For the majority of this work, pentane was the solvent of choice due to its low boiling point and good solubility for the stationary phase. In the case of ov-225 (258 phenyl, 258 cyanopropyl, 508 methylpolysiloxane), pentane was unable to dissolve the polymer and dichloromethane was substituted. 
Table 11. Temperature Effect on the Movement of Solvent,
Meniscus as a Function of Column Length $\left(\beta=115 \times 10^{-5} \mathrm{c}^{-1}\right)$

\section{Column Length}

$10 \mathrm{~m}$

$20 \mathrm{~m}$

$60 \mathrm{~m}$
$\underline{\Delta I^{\circ} \mathrm{C}}$

$12 \mathrm{~mm}$

$24 \mathrm{~mm}$

$72 \mathrm{~mm}$ $\triangle 3^{\circ} \mathrm{C}$

$36 \mathrm{~mm}$

$72 \mathrm{~mm}$

$216 \mathrm{~mm}$ 
In some cases, it was found that ultrasonic agitation decreased the time necessary to dissolve the stationary phase. The film thickness could be calculated from: 202

film thickness $(\mu \mathrm{m})=2.5 \times \mathrm{col}$ i.d. $(\mathrm{mm}) \times$ conc. coating soln $(\mathrm{z})$

This calculation introduces a slight error since the concentration would correctly be given as vol. stat. phase/vol coating solvent but in practical use, it is regarded as weight stationary phase/volume coating solvent and the density of the polymer is regarded as 1.0. Actual values of stationary phase densities have been published and for the majority of cases, this approximation is valid. 203 This relationship shows that as the column i.d. decreases, it is necessary to increase the concentration of the coating solution to maintain a constant film thickness.

\section{Column Filling}

The columns were filled under $\mathrm{N}_{2}$ pressure using a side arm reservoir vessel prepared in-house. The reservoir was made by epoxying a 1/16" stainless steel swagelock tee fitting onto a screw cap which had previously been drilled out to accommodate one arm of the tee (Figure 34). A teflon disc was used to protect the coating solution from the cap material. The screw cap made the reservoirs easily interchangeable and allowed cheap, disposable bottles to be used. The column was held in place by a graphite ferrule which was reusable. A thick walled bottle permitted the use of pressures up to $50 \mathrm{p.s.i}$. to push the solution through the column. For the coating of $50 \mu \mathrm{m} \mathrm{i.d.} \mathrm{columns,} \mathrm{a}$ stainless steel pressure chamber (Figure 35) was used to allow 


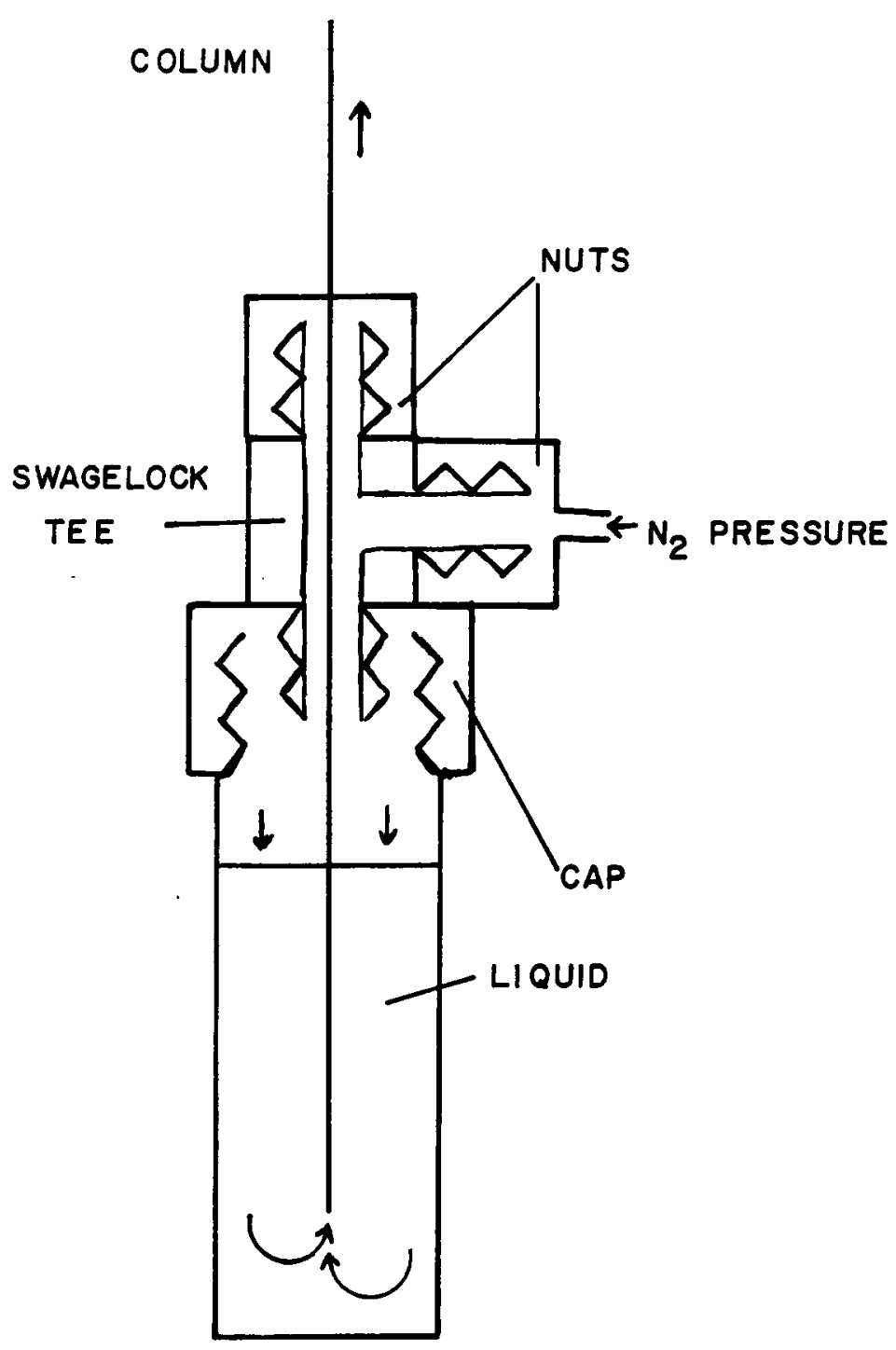

Figure 34. Coating rig for pressurized rinsing and filling of capillary columns using an easily changed and disposable reservoir. 


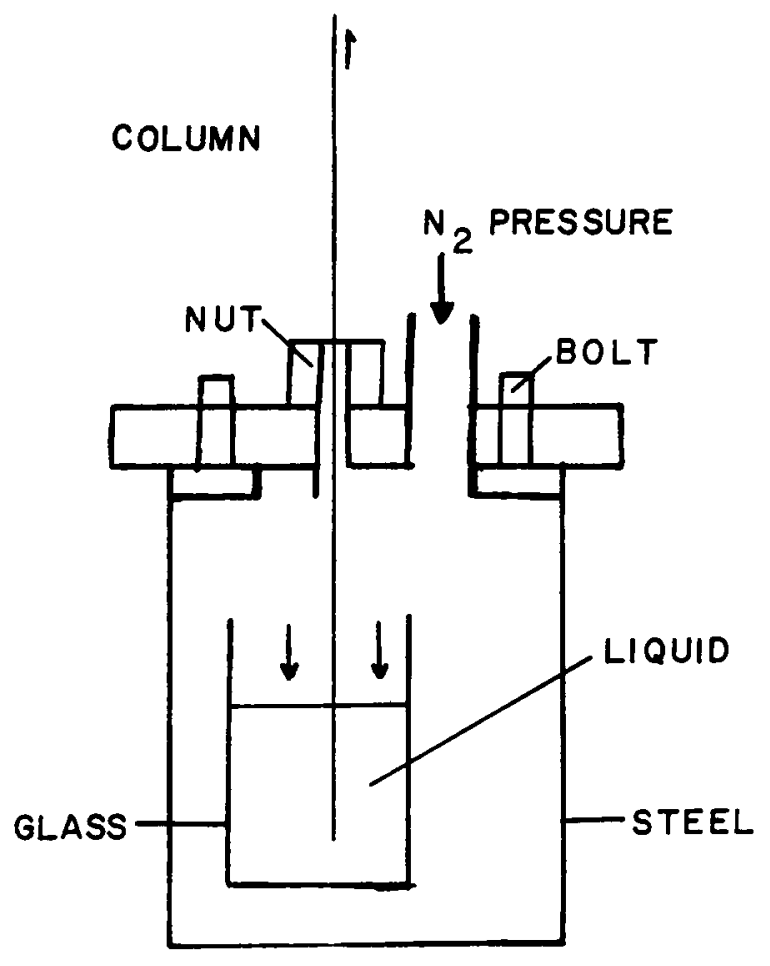

Figure 35. High pressure coating vessel for rinsing and filling small diameter columns. 
operation with $\mathrm{N}_{2}$ pressures up to $250 \mathrm{p.s.i}$. When this chamber was used, the solution was placed in a glass vial in the chamber bottom.

\section{Column End Sealing}

Various methods for sealing the ends of capillary columns have been advocated in the literature. Some examples of materials used include glue, adhesives 204 , paraffin wax 205,206 , and mechanical crimps and closures. 207 Unfortunately, a simple, fast and reliable method does not seem to exist. Ideally, the seal should be made guickly, which excludes steps involving the drying of a glue or the curing of an epoxy. The simplest methods involving insertion of a column end into a grease or gum do not always seal properly either due to failure to seal completely, or to dissolution of the seal material by the coating solvent. After evaluating many methods of end sealing, it was found that the best results were obtained by filling the column with coating solution and then pushing a $30 \mathrm{~cm}$ length of $\mathrm{N}_{2}$ onto the inlet of the column by raising the capillary above the level of coating solvent for a few moments. The exit end of the column was then immersed in iso-octane which was contained in another coating flask at the column exit. A few drops of coating solvent was pushed into the iso-octane from the column to prevent gas bubbles from collecting at the liquid-liquid interface. The iso-octane was then pushed onto the column until the air gap at the column inlet had been displaced. When coating solvent dripped back into the inlet, this indicated that a $30 \mathrm{~cm}$ length of iso-octane was present at the outlet. Another solvent reservoir containing melted paraffin wax 
(Gulfwax) was substituted for the iso-octane reservoir. A few drops of iso-octane were pushed into the hot paraffin after the column was inserted below the level of the melted wax to again eliminate the possibility of bubbles at the interface. The hot paraffin was then pushed onto the column by $\mathrm{N}_{2}$ pressure. The wax cooled in the thin tubing and stopped after about $20 \mathrm{~cm}$ of travel. The inlet was then attached to the vacuum system using an 1/8" swagelock fitting and a graphite ferrule. When the column was evacuated, successful closure was indicated by the smooth movement of the meniscus away from the vacuum line.

When this method was properly performed, a near perfect record was achieved for good closures. Although the closure operation was complex and technique dependent, it was reliable and had the virtue of working with all coating solvents. The iso-octane/paraffin method was easily performed with $100 \mu \mathrm{m}$ i.d. and larger columns, but became difficult to manipulate with smaller columns. For the 50 um i.d. columns, ends were sealed by either submerging the column outlet in Apiezon $N$ grease or in a fast drying epoxy. Both of these methods had the advantages of simplicity, but their use usually resulted in several attempts being made to achieve a good seal.

\section{Evaluation of Crosslinking Reagents For SCF CO 2}

Although dicumyl peroxide at low $(0.58)$ levels is sufficient for. crosslinking non-polar stationary phases, more polar phases are more difficult to crosslink due to fewer methyl protons being available. In 
addition DCP has been reported to oxidize the stationary phase causing increased polarity and decreased stability. Both initiators were evaluated under supercritical $\mathrm{CO}_{2}$ conditions.

\section{Crosslinking of ov-1701}

Two $0.25 \mathrm{~mm} \times 10 \mathrm{~m}$ fused silica columns were rinsed with dichloromethane and statically coated at $30^{\circ} \mathrm{C}$ with $5.4 \mathrm{mg} / \mathrm{ml}$ ov-1701 in $5 \mathrm{ml}$ pentane. One column had $100 \mu \mathrm{L}$ of $28 \mathrm{DCP}$ in toluene dissolved in the coating solution to produce a $48(w / w)$ concentration of the initiator in the stationary phase. Neither column was deactivated so only the effects of crosslinking could be observed.

After coating, the two columns were conditioned under $\mathrm{H}_{2}$ flow by temperature programming from $40^{\circ} \mathrm{C}$ to $160^{\circ} \mathrm{C}$ at a $1^{\circ} \mathrm{C} / \mathrm{min}$ programming rate. The temperature was maintained at $160^{\circ} \mathrm{C}$ for 1 hour and was then raised by $1^{\circ} \mathrm{C} / \mathrm{min}$ to $225^{\circ}$ and held for $2 \mathrm{hrs}$. This step removed residual solvent and low molecular weight components of the phase and was sufficient to thermally initiate crosslinking in the column doped with DCP. After conditioning, the Grob test mixture II was run on both the uncrosslinked column and the column crosslinked with DCP using standard conditions.

The column which was not crosslinked was then purged with vapors of ATB through a $N_{2}$ bubbler and allowed to saturate with these vapors for 1 hour at room temperature. The column ends were then flame sealed and he sealed column was heated from $25^{\circ} \mathrm{C}$ to $190^{\circ} \mathrm{C}$ at $2^{\circ} \mathrm{C} / \mathrm{min}$ and held at $190^{\circ} \mathrm{C}$ for 3 hours to initiate ATB crosslinking. The column was then reconditioned under $\mathrm{H}_{2}$ flow and reevaluated with the Grob mix. 
The two columns, one crosslinked with DCP and the other with ATB, were connected to a syringe pump filled with $\mathrm{CO}_{2}$ and fitted with restrictors. After placement in a gas chromatograph's oven, they were exhaustively extracted with $\mathrm{CO}_{2}$ at pressures up to $2500 \mathrm{psi}$ and temperatures from $25^{\circ} \mathrm{C}$ up to $150^{\circ} \mathrm{C}$. This was continued for a minimum of hours. The columns were then reevaluated with the Grob mix.

\section{Evaluation of a Commercial Column with SFC CO 2}

A commercial crosslinked methyl silicone column was obtained from Hewlett-Packard Co. (Avondale, PA). The column was a 12 meter $0.2 \mathrm{~mm}$ i.d. test column (HP Part \#19091-60312). The column was evaluated using the Grob test mix II and was then extracted with $\mathrm{CO}_{2}$ as in the previous experiments. After extraction, the column was again evaluated with the test mix to monitor column changes.

\section{Evaluation of Polyethylene Glycol Deactivation}

One of the initial concerns in this investigation was the effects of supercritical $\mathrm{CO}_{2}$ on the stationary phase. The swelling of polymeric phases during solvent rinsing can lift the phase off the wall without dissolution. If the deactivation layer is unstable in the $\mathrm{CO}_{2}$, the possibility that the column will deteriorate is increased. In order to evaluate the utility of semi-polar liquid phases for SFC, the deactivation method and the cross-1inking method must be tested under supercritical conditions.

For the initial work, column evaluation was conducted using $0.25 \mathrm{~mm}$ i.d. columns. These column diameters are standard for gas 
chromatography yet are still suitable for operation at the high pressures of SFC. The larger column sizes and capacities allow more detailed information to be gathered on the effects of supercritical $\mathrm{CO}_{2}$. When this work was begun, only very non-polar phases such as oV-1 (1008 methyl polysiloxane) and SE-54 (58 phenyl 958 methylpolysiloxane) had been successfully utilized with SFC. Since the selectivity in chromatography is maximized when the polarity difference between the mobile and stationary phase is greatest, the potential of SFC with $\mathrm{CO}_{2}$ is greatest with more polar columns. Unfortunately, very polar columns are extremely difficult to both coat and crosslink even for GC applications so semi-polar phases were chosen for this SFC study. Semipolar phases also provide the greatest challenge for deactivation. As discussed earlier, excellent methods for deactivation of non-polar methy.l silicones have been developed and polar phases tend to be selfdeactiving since they tend to cover active sites. Semi-polar phases form the real test for deactivation methods.

To evaluate the efficiency and stability of the Carbowax 20M deactivation, a phase prepared in our laboratory was used. This polymer was a 258 phenyl, 18 vinyl, 748 methyl polysiloxane referred to as $\mathrm{HB}-1$. Columns were prepared with and without the deactivation treatment so that a valid comparison could be made. The deactivated column was prepared by rinsing a 10 meter length of fused silica with 1 column volume of methanol followed by 1 column volume of dichloromethane. A 28 solution of Carbowax $20 \mathrm{M}$ in dichloromethane was slowly pushed through the column with $\mathrm{N}_{2}$ pressure to dynamically coat the column. After 1 
column volume of the deactivation solution was passed through the column, the column was purged with dry $\mathrm{N}_{2}$ to remove residual solvent. The ends of the column were then sealed and the column was heated at $280^{\circ} \mathrm{C}$ for 16 hours. The column was then cooled to room temperature and rinsed with 1 column volume of dichloromethane to remove decompositon products. The column which was not deactivated was rinsed with methanol followed by dichloromethane.

The two columns were then statically coated by filling the column with a $4.9 \mathrm{mg} / \mathrm{ml}$ solution of $\mathrm{BB}-1$ in $10 \mathrm{ml}$ pentane. A 28 solution of dicumylperoxide (DCP) in toluene was prepared and $10 \mu 1$ was added to the coating solution to give a peroxide concentration of 0.58 . The column was immersed in a double wall contant temperature bath and after equilibration, one end was sealed. The other end was attached to vacuum.

Following the coating, the columns were placed in Hewlett-Packard $5880 \mathrm{~A}$ (Avondale, PA) a gas chromatograph under $\mathrm{H}_{2}$ flow. The temperature was programmed at $1^{\circ} \mathrm{C} / \mathrm{minute}$ up to $200^{\circ} \mathrm{C}$ and held for 2 hours. After conditioning the columns were evaluated for efficiency and activity using an isothermal analysis of a hydrocarbon sample and the Grob Test Mix II. Following testing, the columns were extracted with $\mathrm{CO}_{2}$ for 4 hours at $50-100^{\circ} \mathrm{C}$ and pressures up to $3500 \mathrm{psi}$ and then again evaluated.

\section{Reproducibility of Capillary SFC}

\section{Column Activation and Deactivation}

Three $12 \mathrm{~m} \times 100 \mu \mathrm{m}$ i.d. columns were prepared in an identical manner except for the activation/deactivation treatment. The first column was 
prepared for coating by rinsing with dichloromethane. The remaining two columns were hydrothermally activated with nitric acid prior to deactivation by the following procedure.

One column volume of $208 \mathrm{HNO}_{3}$ was washed through the column using nitrogen pressure. After the plug had been pushed out of the column, the ends of the column were flame sealed. The column was placed in a gas chromatographic oven and the temperature was programmed at $15^{\circ} \mathrm{C} /$ minute up to $200^{\circ} \mathrm{C}$ and maintained for 10 hours. After cooling, the column was rinsed with $1 \mathrm{ml}$ of $18 \mathrm{HNO}_{3}$ followed by $1 \mathrm{ml}$ of methanol. The column was then dried at room temperature under dry $\mathrm{N}_{2}$ flow to remove residual solvent before deactivation.

The two activated columns were deactivated by different methods. The first was dynamically coated with a dilute 28 solution of superox 20M in dichloromethane. After a column volume had passed through the column, the column was held at room temperature under dry $\mathrm{N}_{2}$ flow to remove residual solvent. The column ends were then sealed and the column was heated at $280^{\circ} \mathrm{C}$ for 16 hours. Decomposition and pyrolysis products were then removed by rinsing with dichloromethane. After drying under $\mathrm{N}_{2}$ flow the column was ready for coating.

The second activated column was dynamically coated with a solution of mixed cyclic siloxanes. The mixture was prepared so that the mole percent of each functionality in the cyclic material would equal that in the stationary phase used to coat the column. For ov-1701, this would result in a 78 phenyl, 78 cyanopropy 1868 methyl polysiloxane. Unfortunately, cyclic siloxanes containing cyanopropyl groups were 
unavailable so a cyanoethyl cyclic siloxane was substituted. This mixture of cyclic siloxanes was very viscous and the solid cyclics, the phenyl and cyanoethyl, were not totally soluble in the liquid $\mathrm{D}_{4}{ }^{\text {. To }}$ decrease viscosity and dissolve the components the mixture of cyclics was diluted $1: 1 \mathrm{v} / \mathrm{v}$ with dichloromethane. A column volume of this solution was then passed through the column. After the plug had passed, the column was dried under $\mathrm{N}_{2}$ flow to remove residual solvent. At this point the column ends were sealed and it was heated to $400^{\circ} \mathrm{C}$ for 15 hours to initiate the cyclic ring opening reaction. Longer periods of heating at this temperature resulted in oxidation of the polyimide outer coating resulting in first darkening of the column and then degradation of the outer coating. This led to very fragile columns which broke so easily that the time at $400^{\circ} \mathrm{C}$ was limited to 1.5 hours. After cooling the column was rinsed with dichloromethane to remove any unbound material after drying under $\mathrm{N}_{2}$ flow the column was ready for coating.

\section{Surface Energy Measurements}

The surface energy of columns deactived by Superox $20 \mathrm{M}$ pyrolysis was previously reported by ogden to be 44 dynes/cm, but a mixed cyclic of this particular composition had not yet been reported. ${ }^{216}$ A $5 \mathrm{~m}$ length of $0.2 \mathrm{~mm}$ fused silica tubing was hydrothermally activated with $\mathrm{HNO}_{3}$ and then deactivated using the cyclic procedure described above. The capillary was then sectioned into pieces suitable for analysis and the height of liquid rise was measured using the apparatus in Figure 36. The liquids used were a series of methanol/water mixtures whose physical constants are given in Table 8 . The capillary column was positioned 
vertically in the apparatus with the lower end extending into the 1iquid. The height of capillary rise was measured when the upward motion of the liquid had ceased. This normally required at least 10 minutes for equilibration. After each measurement, the length of column which had been wet was clipped off and a fresh section was used for the next measurement. Repeated measurements in the same liquid showed the energy of the column to be uniform throughout its length. Only the advancing contact angle was measured

From the construction of a $z$ isman plot (Figure 37 ), the critical surface energy of the column was found to be 28 dynes/cm which indicates that the surface formed is not methylated and should be suitable for phase wettability by ov-1701. This is of importance since some controversy has developed over claims that functionalities such as phenyls and methyls on the surface at high temperatures degrade to form only a methylated surface. 217 This assertion has been challenged chromatographically and by the results of wettability measurements. $216,218,219$ From the surface energy measurements, good coating with ov-1701 could be expected and was indeed observed with the cyclic mixture.

\section{Coating and Evaluation Procedure for Reproducibility Study}

The columns were statically coated in a doubly insulated water bath held at $30^{\circ} \mathrm{C}$. The $\mathrm{ov}-1701$ was dissolved in pentane at a concentration of $0.01 \mathrm{~g} / \mathrm{ml}$ to give a film thickness of $0.25 \mu \mathrm{ms}$. An iso-octane paraffin seal was used to provide leak tight closure of the column. The columns were subjected to vacuum overnight to insure complete removal of 


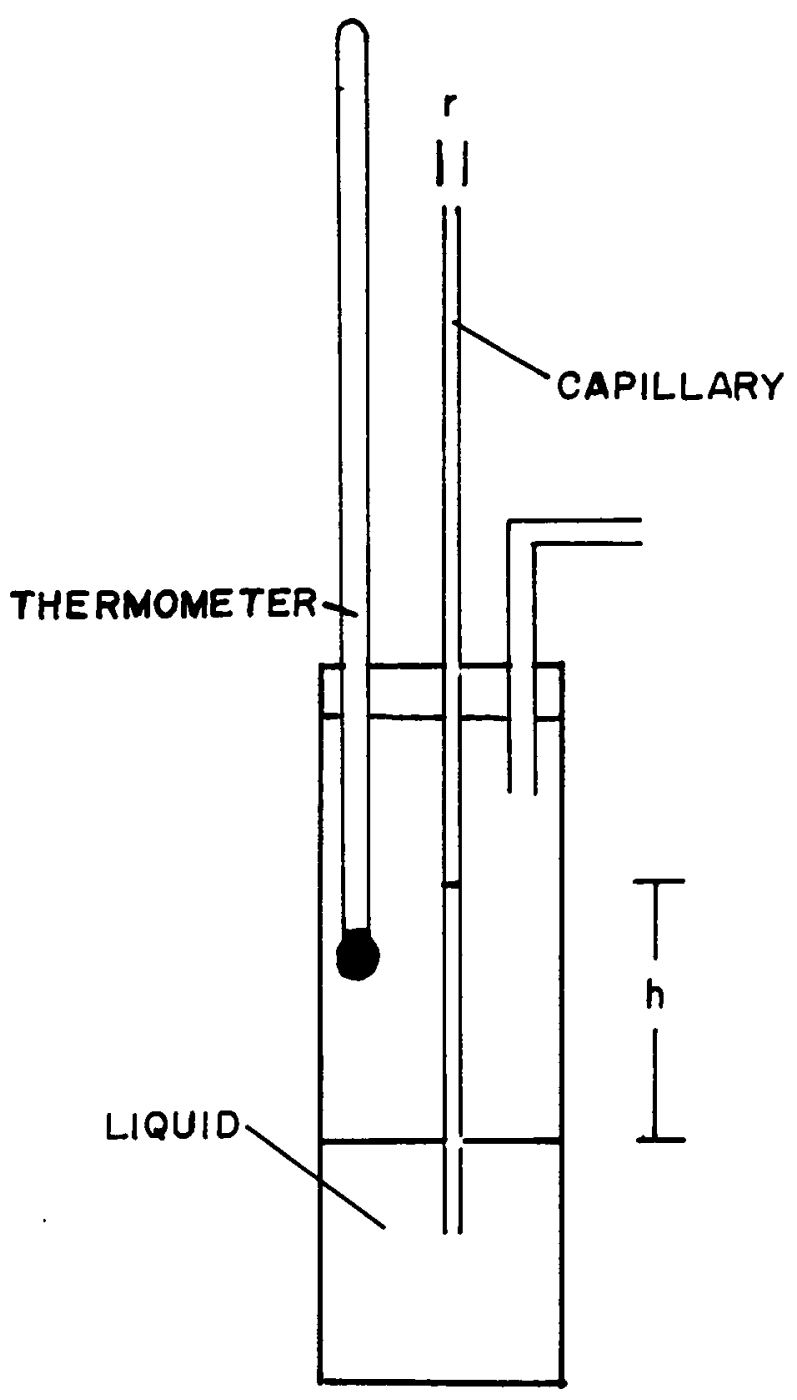

Figure 36. Apparatus for measurement of capillary rise. 


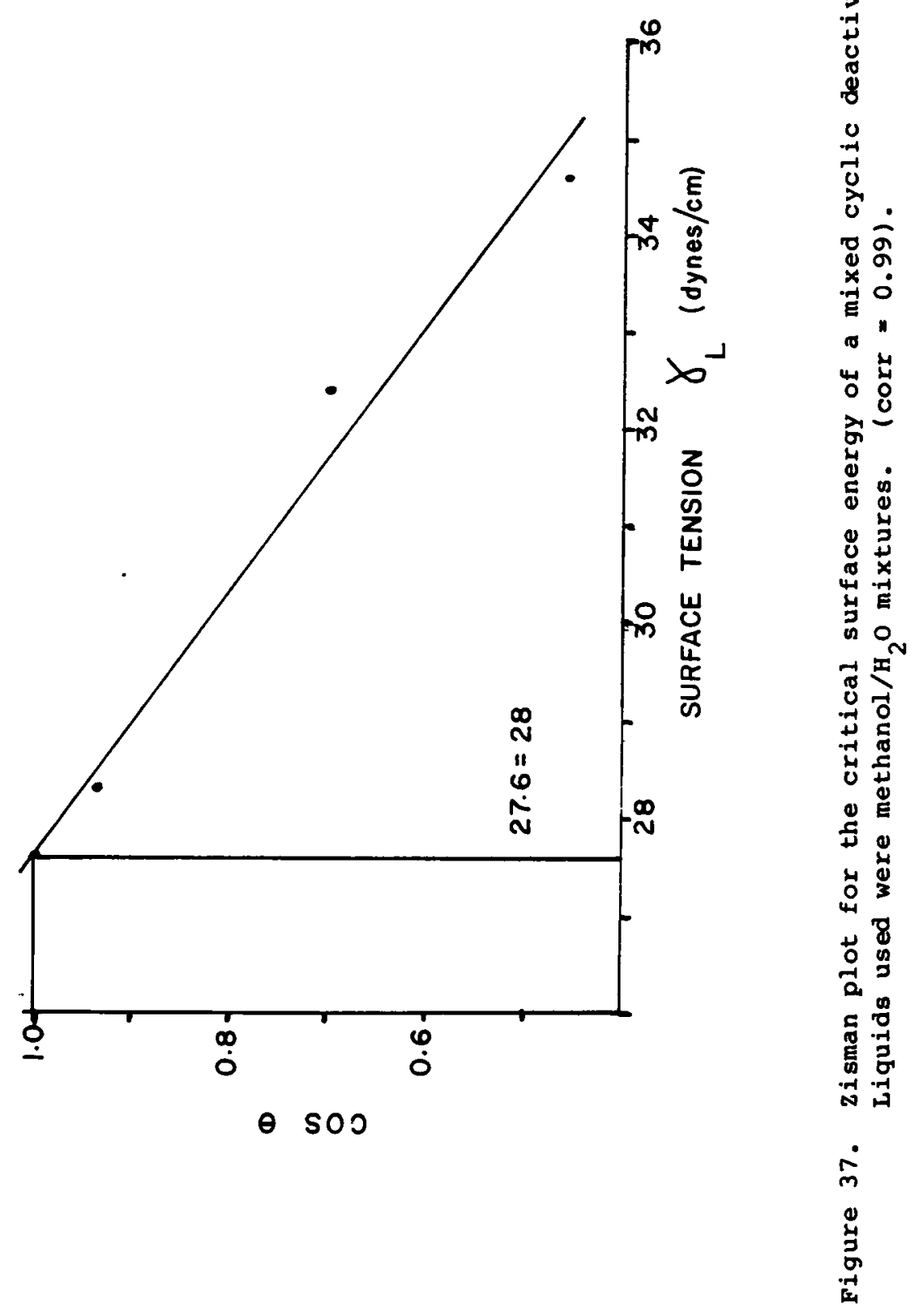


coating solvent. Crosslinking was accomplished by purging the column with ATB vapors, flame sealing the ends and heating at $200^{\circ} \mathrm{C}$ for 3 hours. It was found experimentally that the columns must be conditioned under flow at $200^{\circ} \mathrm{C}$ for a least 30 minutes before crosslinking or the columns would plug during crosslinking and no flow could be forced through the column even at high pressures.

After crosslinking the columns were installed in the SFC chromatograph and conditioned with $\mathrm{CO}_{2}$ at $100^{\circ} \mathrm{C}$ and pressures up to 5000 psi. The peak symmetry of the paraffin mix was used to determine if the column connections were properly made. Test mixtures were prepared using normal alkanes from paraffin, linear unsaturated alcohols and linear aliphatic acids. The paraffin test mix was obtained by dissolving Gulfwax in dichloromethane at a concentration of $10 \mathrm{mg} / \mathrm{ml}$. The alcohol test mix and the acid test mix were prepared by dissolving each component at a concentration of $0.4 \mathrm{mg} / \mathrm{mL}$ in dichloromethane. An exception was the $\mathrm{C}-24$ alcohol which was present at $0.2 \mathrm{mg} / \mathrm{mL}$. This gave a $12 \mathrm{ng} /$ component on column for each component (long for the $\mathrm{C}-24$ alcohol) with the $0.1 \mu \mathrm{L}$ loop and a $2: 1$ split ratio.

The mixtures were chromatographed at $100^{\circ} \mathrm{C}$ starting at 1100 p.s.i. and pressure programming to $5000 \mathrm{psi}$ at $125 \mathrm{psi} / \mathrm{min}$. 
CHAPTER VI

RESULTS AND DISCUSSION

\section{Introduction}

The first necessity for a SFC column is the ability to resist dissolving in the supercritical fluid. The results of an evaluation of crosslinking initiators are presented first, followed by an evaluation of the stability of the polyethylene glycol deactivation layer. The lessons learned in these studies will then be evaluated in terms of the quantitative reproducibility of the SFC analysis.

\section{Evaluation of Crosslinking}

The major observation from these data is the reduction of film thickness after $\mathrm{CO}_{2}$ extraction that is seen with the DCP crosslinking. A crosslinking efficiency of about 908 is achieved, resulting in a 108 phase loss. Similar treatment of the ATB crosslinked phase resulted in no phase loss.

A summary of the results shown in the chromatograms is given in Table 12 along with results of column efficiency measurements. Despite the fact that column deactivation was not performed as part of this study, the results of the Grob test are not really too bad. One unanticipated effect is the partial deactivation of the column by the DCP. A comparison of the peroxide crosslinked column (Figure 38) and the uncrosslinked column (Figure 39) shows that the DCP crosslinked deactivated column exhibits less adsorption of the diol and the acid since the uncrosslinked column fails to elute the acid. The DCP crosslinked column is, however, quite acidic and does not elute the 


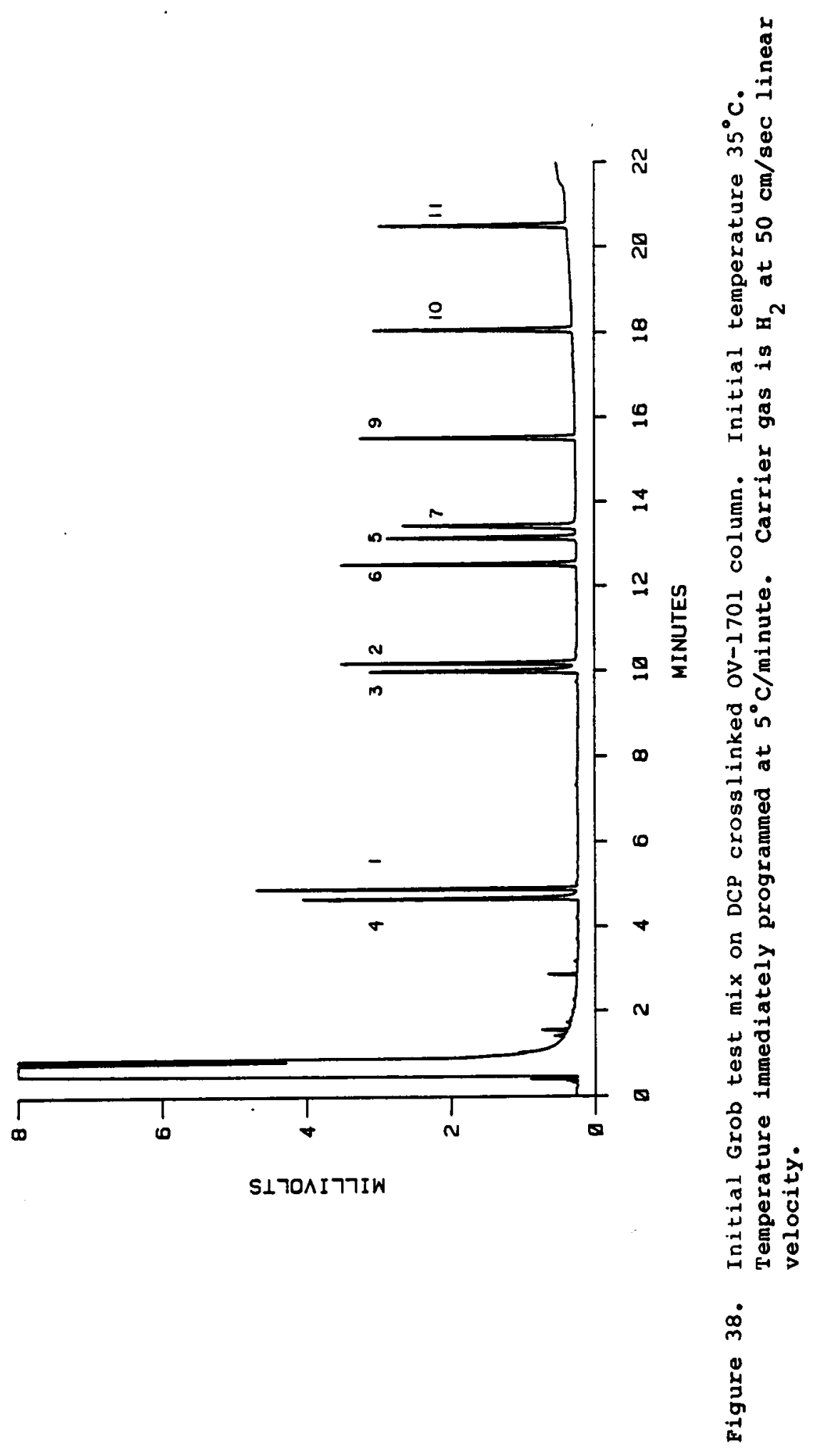




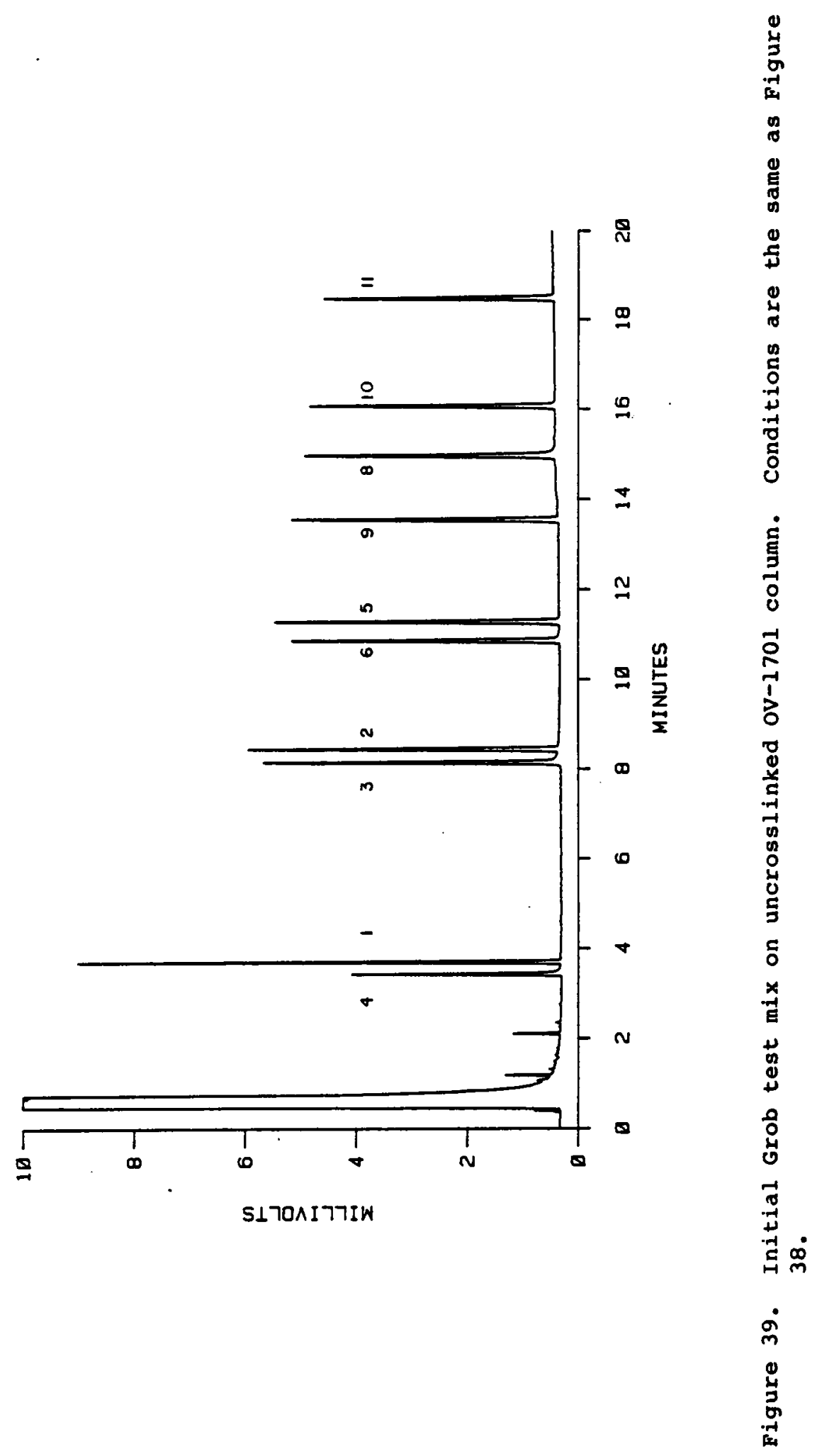




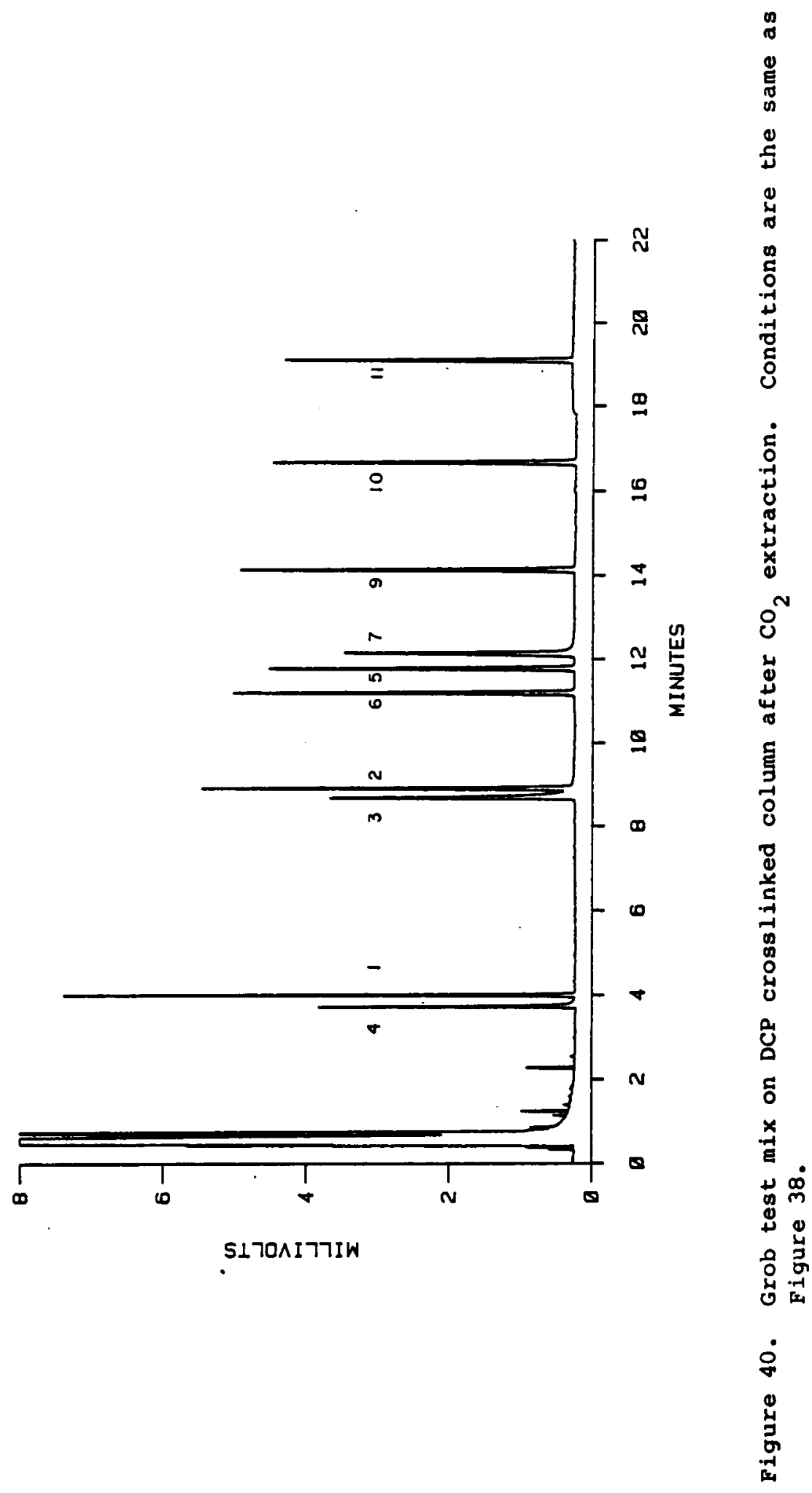




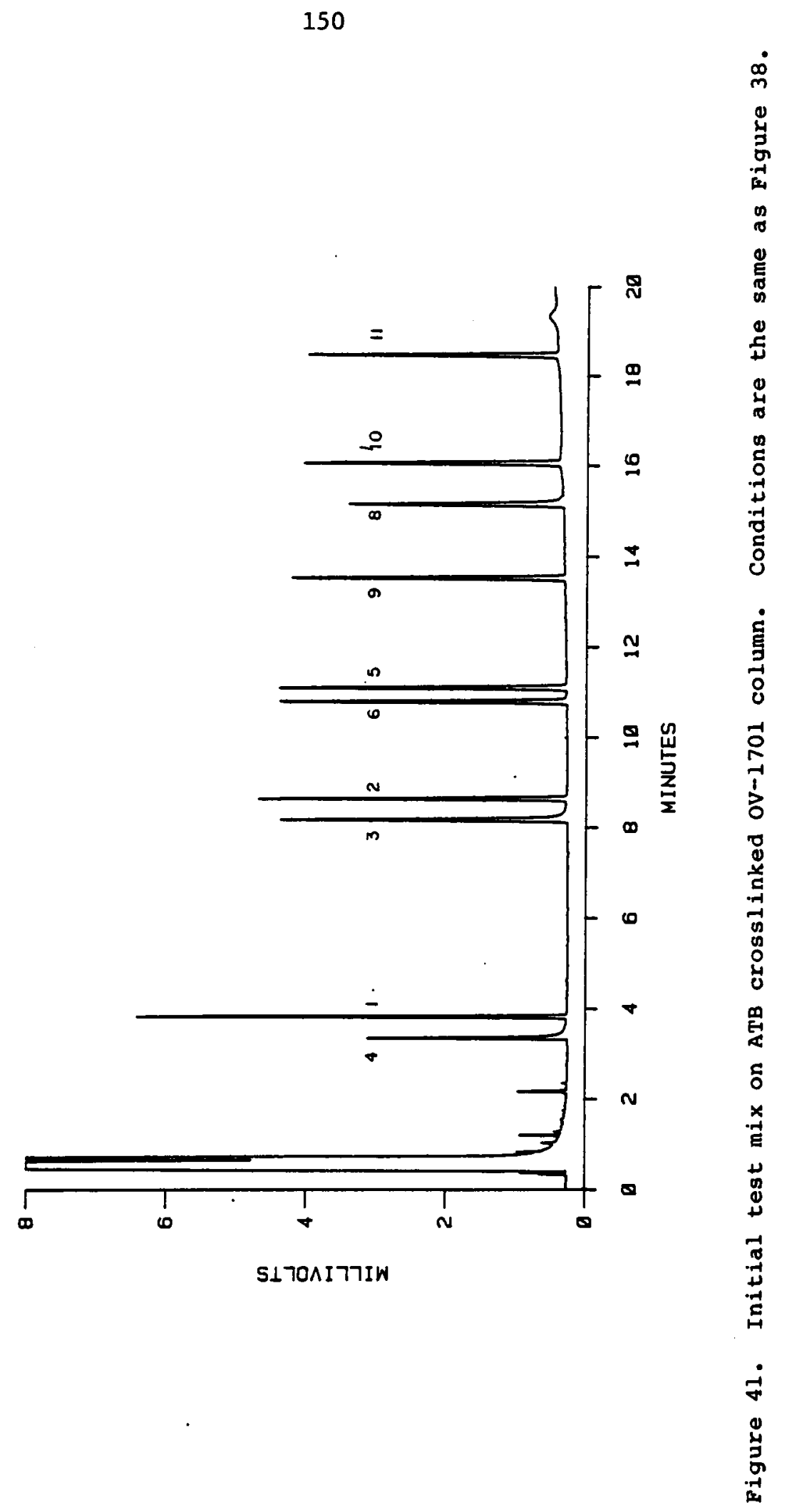




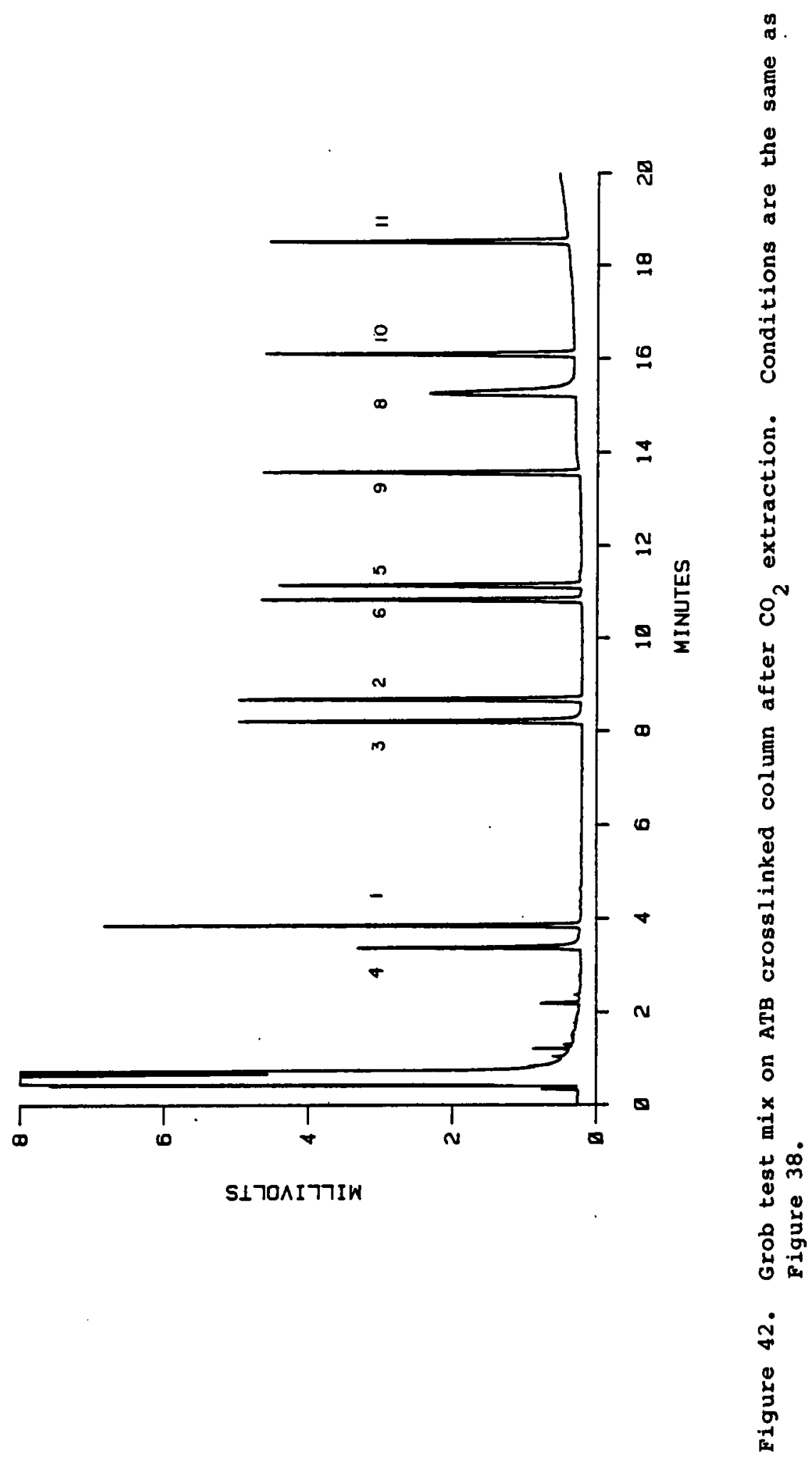


Table 12. Results of a Comparison of Columns Coated with ov-1701 and Crosslinked with ATB or DCP showing

The Results of Extraction with SFC $\mathrm{CO}_{2}{ }^{a}$

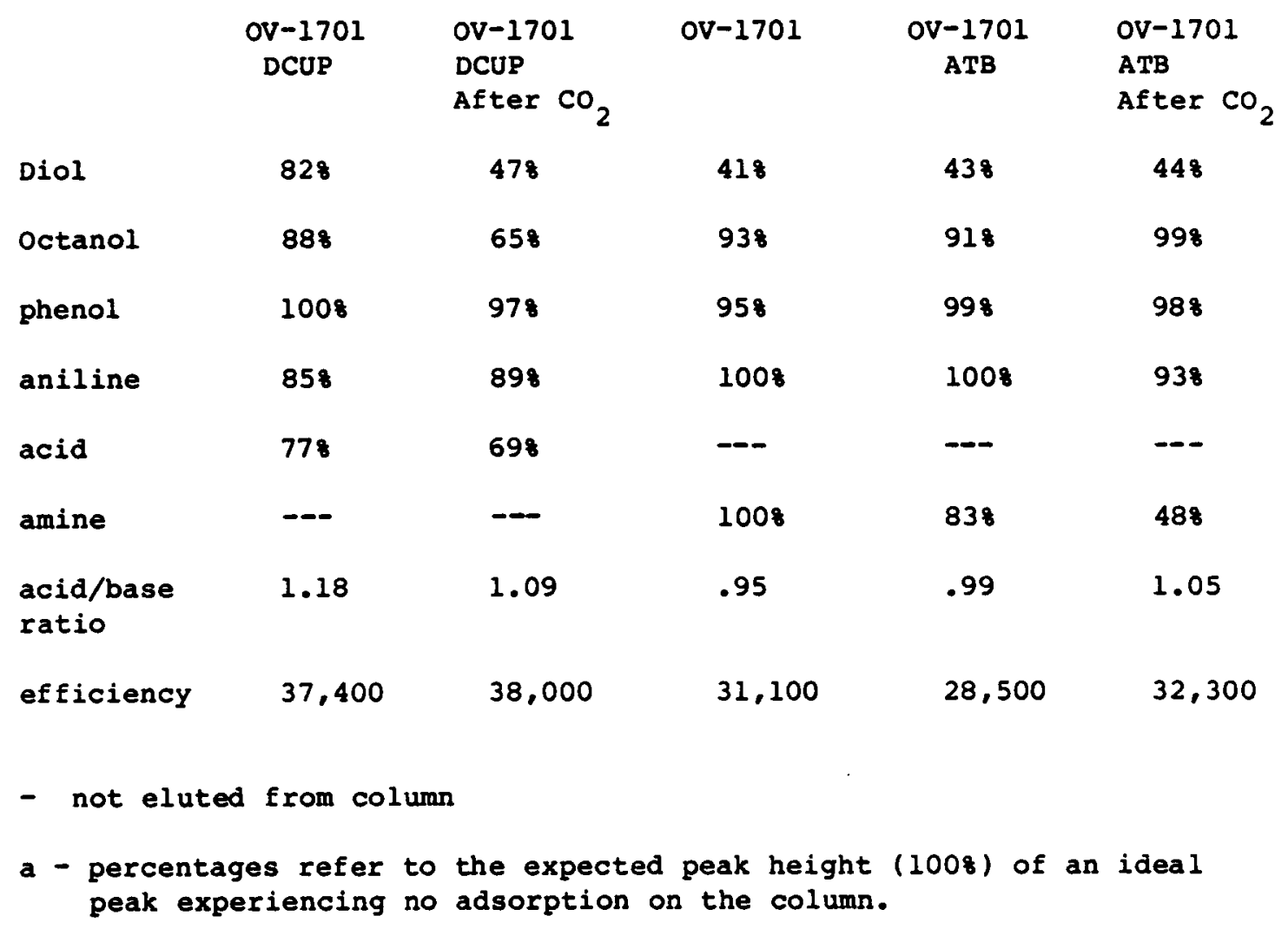


amine. The deactivation caused by the DCP is temporary since extraction with SCF $\mathrm{CO}_{2}$ causes increased adsorption of polar analytes (Figure 40). Presumably, the $\mathrm{CO}_{2}$ removes acidic by-products of the crosslinking reaction resulting in a decrease in column acidity (as indicated by the decreased acid-base ratio) resulting in an increase in the column's adsorptive activity as active column sites are exposed.

Crosslinking with ATB does not result in appreciable changes in column activity or acidity (1.18 to 1.09 ) (Figure 41). The amine does show some increased adsorption, but the overall activity of the column is unchanged. Extraction with $\mathrm{CO}_{2}$ shows a slight increase in column acidity leading to increased adsorption of the basic amine (Figure 42 ).

\section{Results of Evaluation of a Commercial Column With SFC CO 2}

The commercial column was fairly inert, which should be expected for a deactivated column coated with a non-polar methyl silicone. All components in the test mix eluted, although lack of solubility in the non-polar phase caused a distorted peak for the acid (Figure 43). A slight increase in column activity is shown by a decreased peak height for octanol following $\mathrm{CO}_{2}$ extraction (Table 13). In addition, the acid base ratio shows a decreased acidity $(0.97$ to 0.90$)$ after $\mathrm{CO}_{2}$ treatment (Figure 44). The column is slightly basic which is unusual for fused silica columns which normally are neutral to acidic.

The major effect of the $\mathrm{CO}_{2}$ is again a decreased film thickness after extraction. Approximately 108 of the stationary phase is extracted by the $\mathrm{CO}_{2}$ treatment. The increased efficiency shown in Table 15 is probably due to the more favorable diffusion in a thinner film. 
Table 13. The Effect of SCF $\mathrm{CO}_{2}$ Extraction on the Activity and Efficiency of a Commercial Crosslinked Methyl

silicone column $(12 \mathrm{~m} \times 0.2 \mathrm{~mm}$ i.d.)

\section{Compound}

diol

octanol

phenol

aniline

acid

amine

acid/base

ratio

efficiency (N)

\section{Before $\mathrm{CO}_{2}$ Extraction}

888

898

948

978

538

0.97

38,200

\section{After $\mathrm{CO}_{2}$ Extraction}

868

768

908

1008

688

0.90

43,000

eluted with distorted peak shape due to lack of solubility in stationary phase 


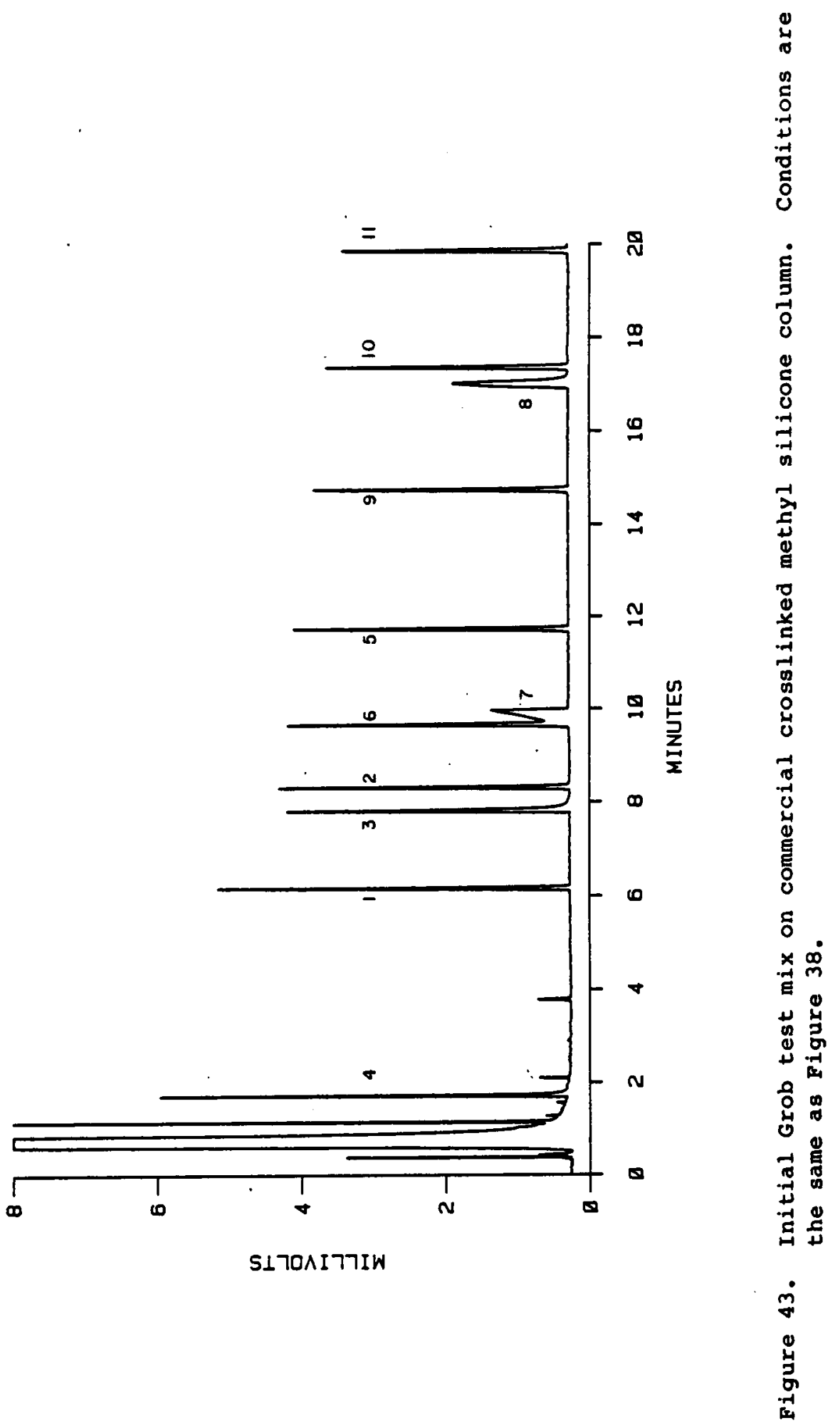




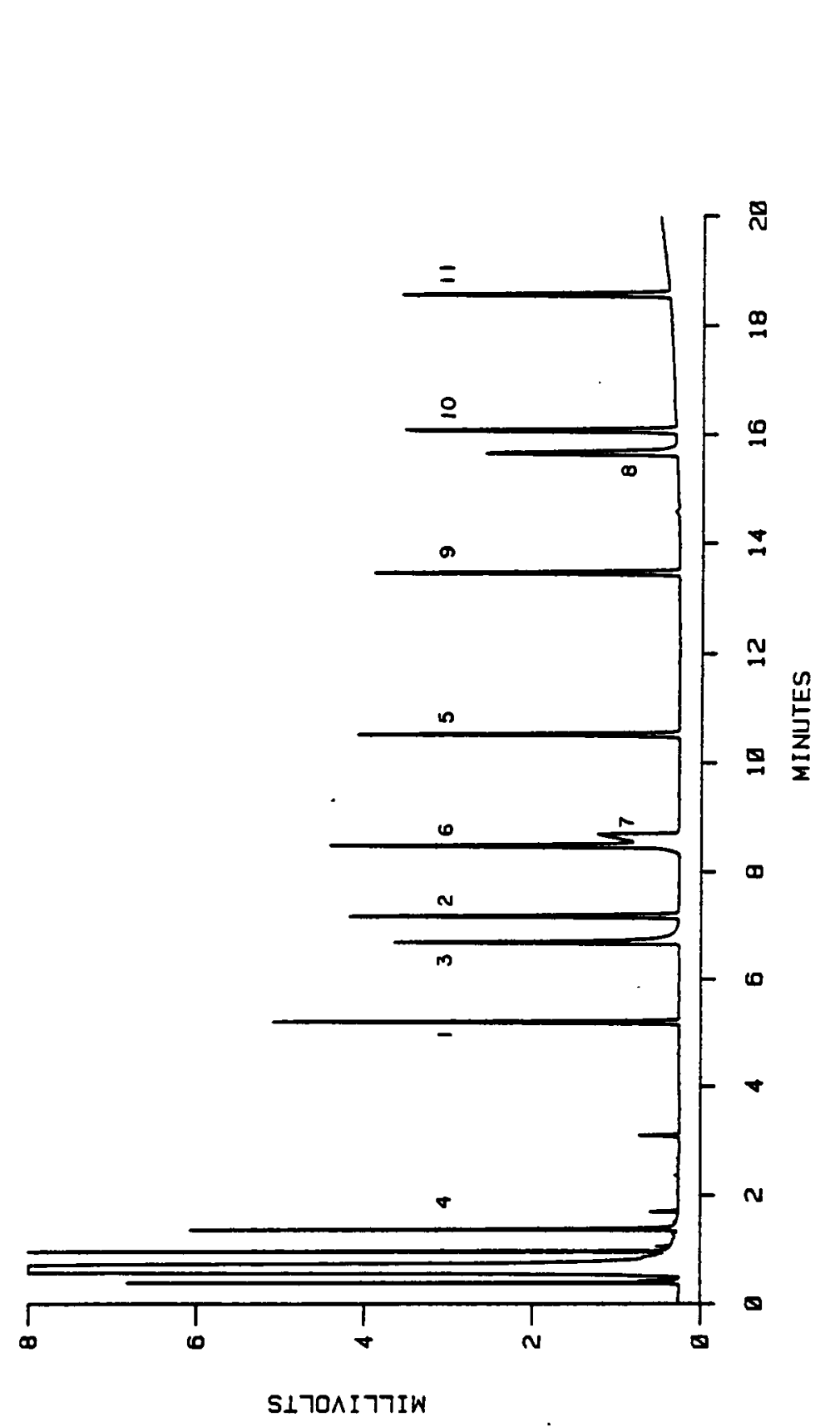

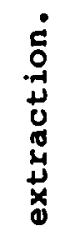

$8^{2}$

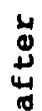

동

䒾

范

肴

on

ㅇํㄱ

ठั.

1

of

0

包

요

도

$\circ$

줄

4

o

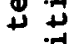

운

ํㅓㅇ

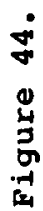


Results of Polyethylene Glycol Deactivation

Both columns coated efficiently with over 30,000 theoretical plates obtained. However, a major difference in column activity was observed. Figure 45 shows the Grob Test Mix on the undeactivated column while Figure 46 shows the same sample under identical conditions on the Carbowax 20M deactivated column. A summary of the values for active components in the Grob test $\mathrm{mix}$ is given in Table 14.

The deactivated column shows elution of all the sample components. The 2-ethyl caproic acid has a peak area consistent with the amount injected although the "fronting" shape of the peak is characteristic of a lack of solubility in the stationary phase. The dicyclohexylamine shows a slightly decreased (828) height and some tailing which is not unexpected for a strong base on a fused silica surface. The 2,3 butanediol is reduced in height (598) which indicates some unreacted surface hydroxyls but deactivation is still excellent as the 1-octanol is eluted without tailing and at full height.

The column which was not deactivated shows major problems. Complete disappearance of the diol and free acid indicates high column activity. Column activity is also indicated by the extreme tailing of the amine and the reduced height and tailing of all polar compounds including the octanol (548). Even the fatty acid methyl esters exhibit slight tailing due to column activity.

Following continued extraction with supercritical $\mathrm{CO}_{2}$, some changes in column activity were noted. Both columns showed decreased adsorption following $\mathrm{CO}_{2}$ treatment. The most dramatic results were seen in the undeactivated column (Figure 47). The diol peak began to reappear 


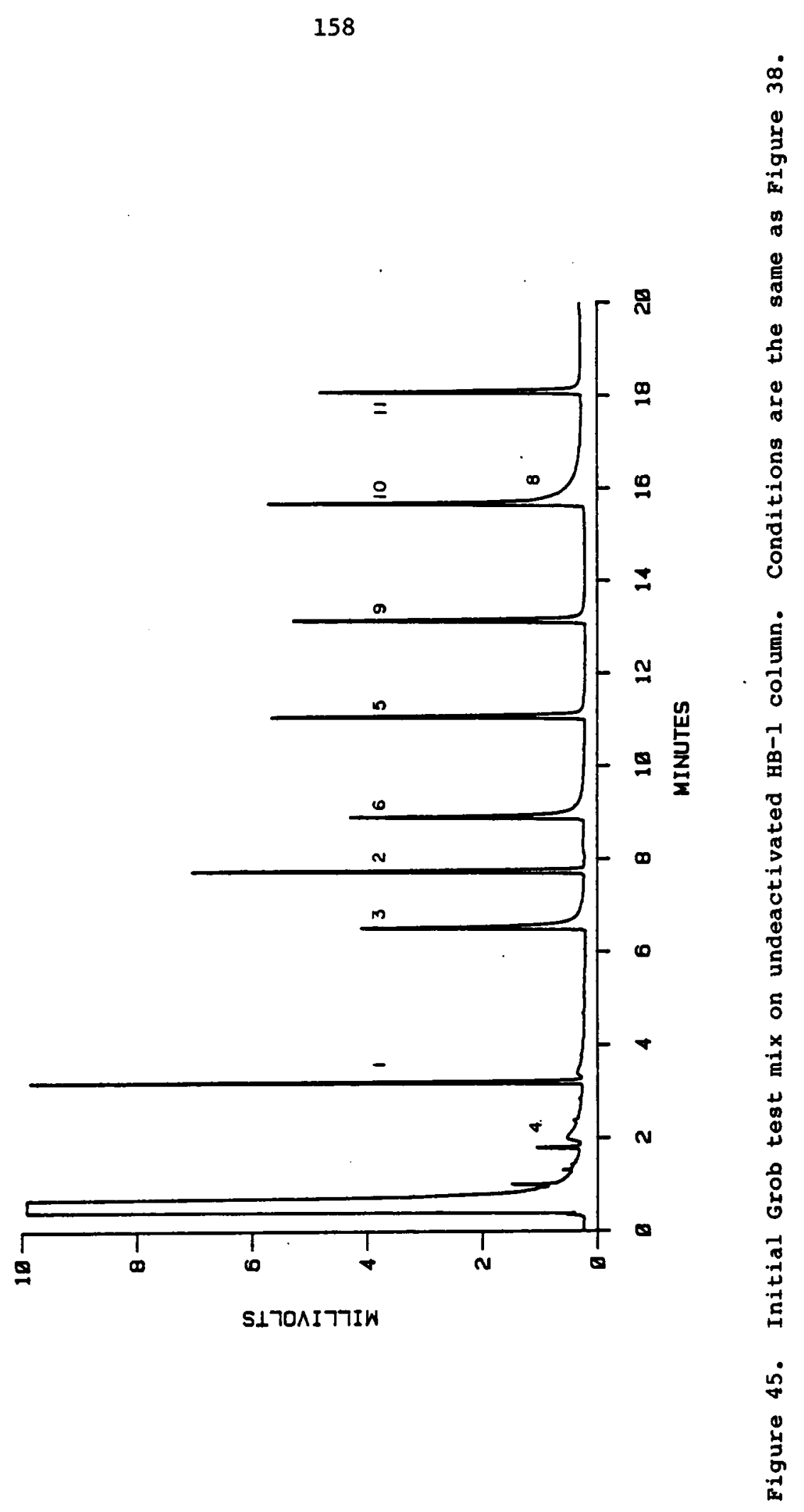




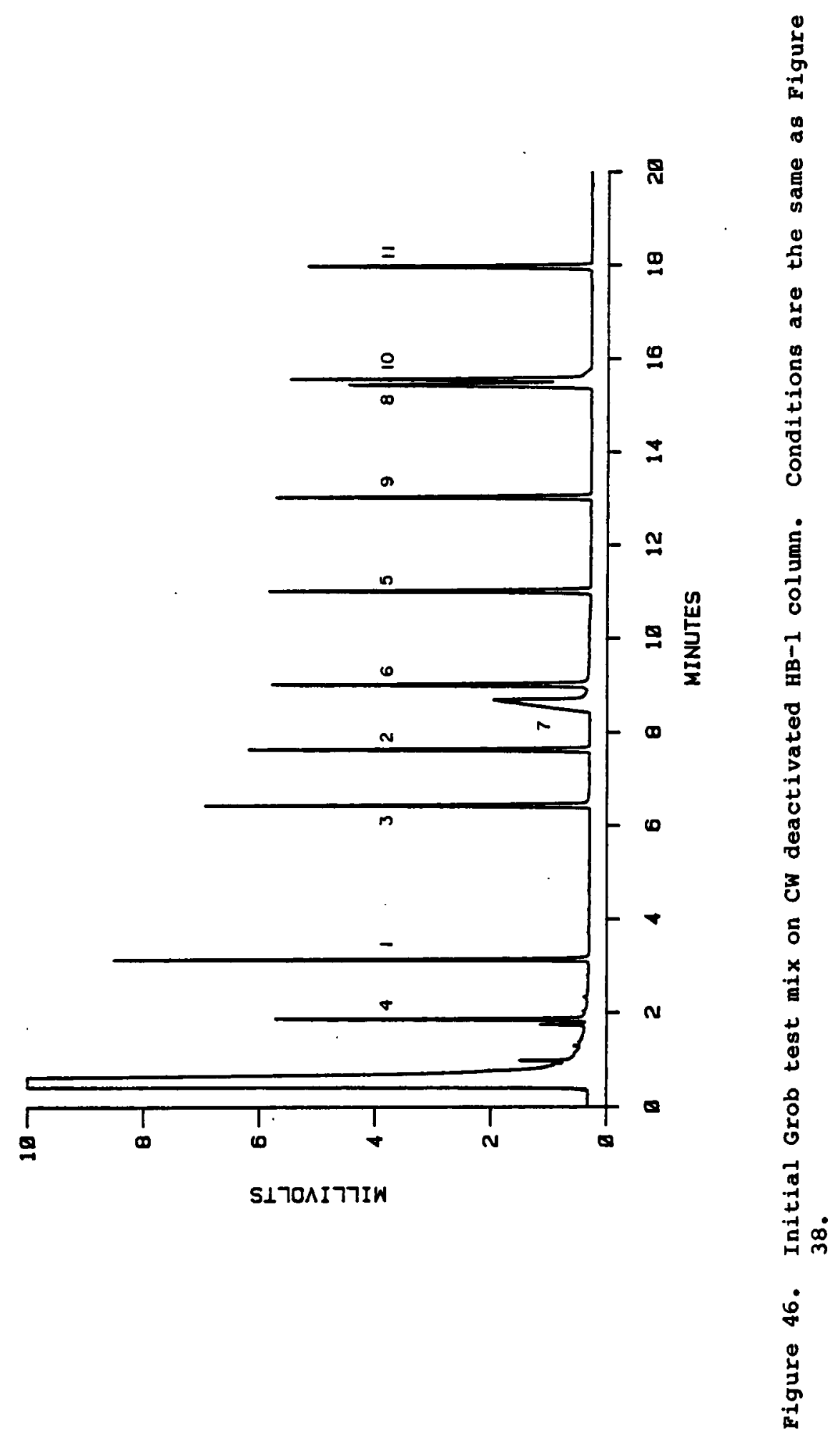


Table 14. Comparison of a Carbowax 20M Deactivated HB-1 Coated Column to an Undeactivated Column Before and After Use with Supercritical Fluid $\mathrm{CO}_{2}{ }^{\mathrm{a}}$ $\begin{array}{lll}\mathrm{HB}-1 & \mathrm{HB}-1 \mathrm{CO}_{2} \quad \mathrm{HB}-1 \text { Deact. } & \mathrm{HB}-1 \text { Deact. } \\ & \text { After } \mathrm{CO}_{2}\end{array}$

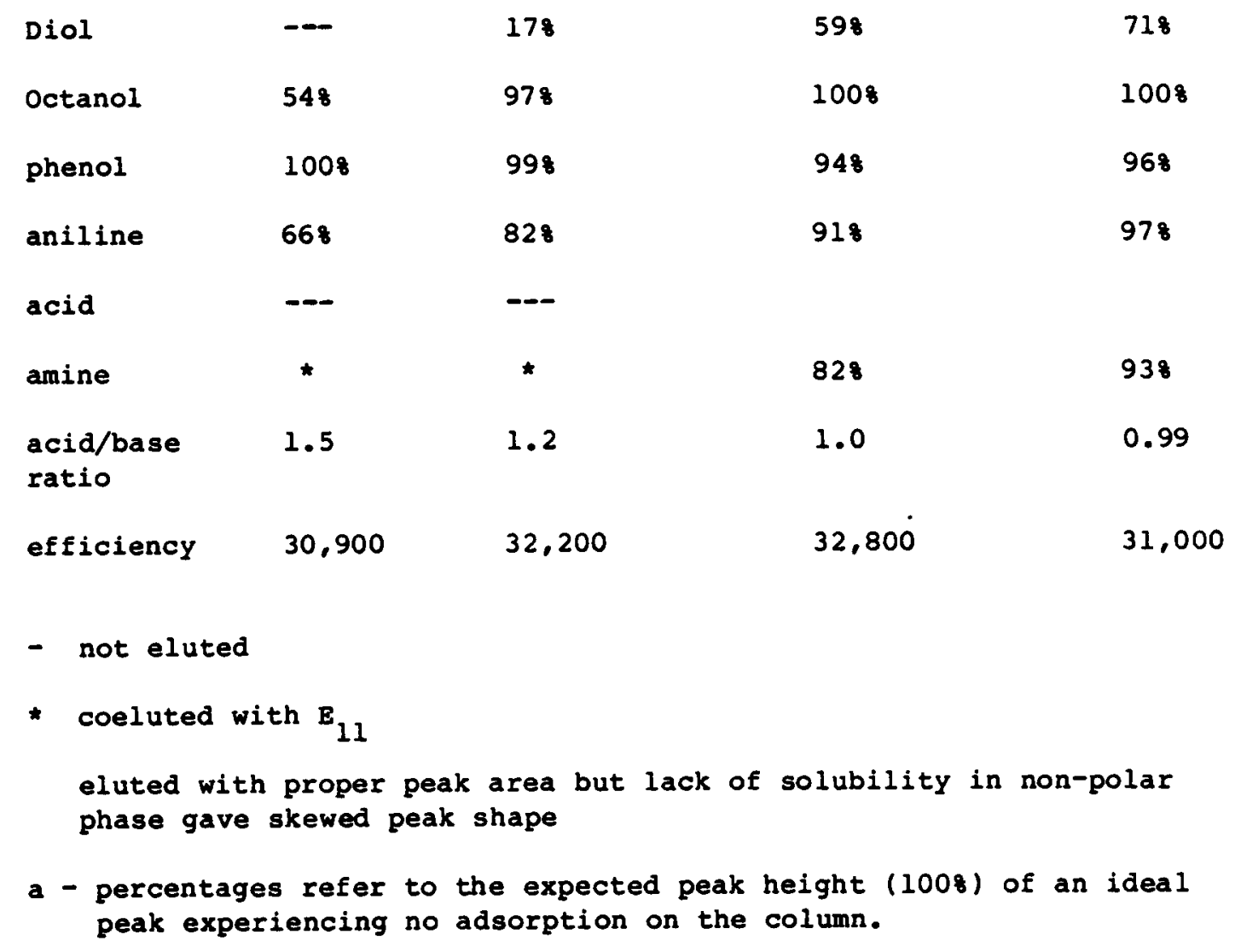


161

although it achieved only 178 of it's expected height. The disappearance of active sites is also noted in the increase in octanol height from 548 to $97 \%$. Aniline also shows a slight improvement. The very polar acid still is completely adsorbed by the column and the amine tails badly as it coelutes with the c-ll methyl ester.

The deactivated column shows some improvement with increased elution of the diol (up to 718 from 598) and the amine (from 828 to 938 ) (Figure 48). The important point is that there has been no decrease in retention time from loss of liquid phase loss and the efficiencies of the columns before and after treatment with $\mathrm{CO}_{2}$ remain practically unchanged. This data indicates that 0.58 DCP is sufficient to crosslink this phase and also indicates that the carbowax deactivation layer is a stable film which does not allow liquid phase stripping. One measure of column inertness is the ratio of the heights of the acidic phenol to the basic aniline. It is seen in Table 14 that the deactivation layer shields the acidic silica surface and produces a more neutral column $($ ratio $=1.0)$. The decrease in column acidity following $\mathrm{CO}_{2}$ treatment may be due to the removal of decomposition products of the dicumyl peroxide.

Kovat's Retention Index

The influence of the pyrolyzed Carbowax $20 \mathrm{M}$ deactivation layer on column selectivity has been given as a reason not to use the technique. 148 However, as the polarity of the stationary phase increases, the difference attributed to the deactivation layer decreases. To investigate the influence of the deactivation layer, 


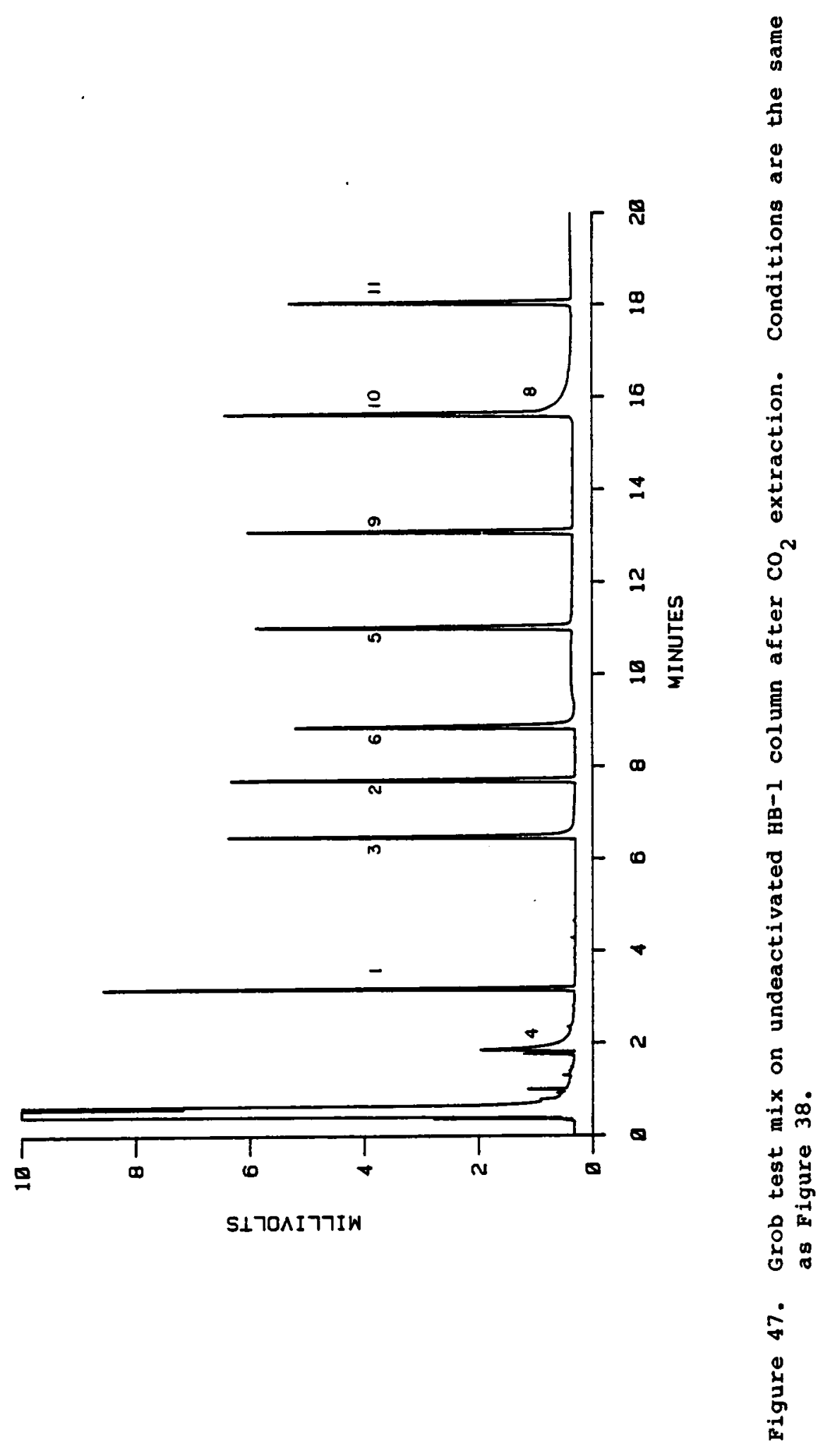




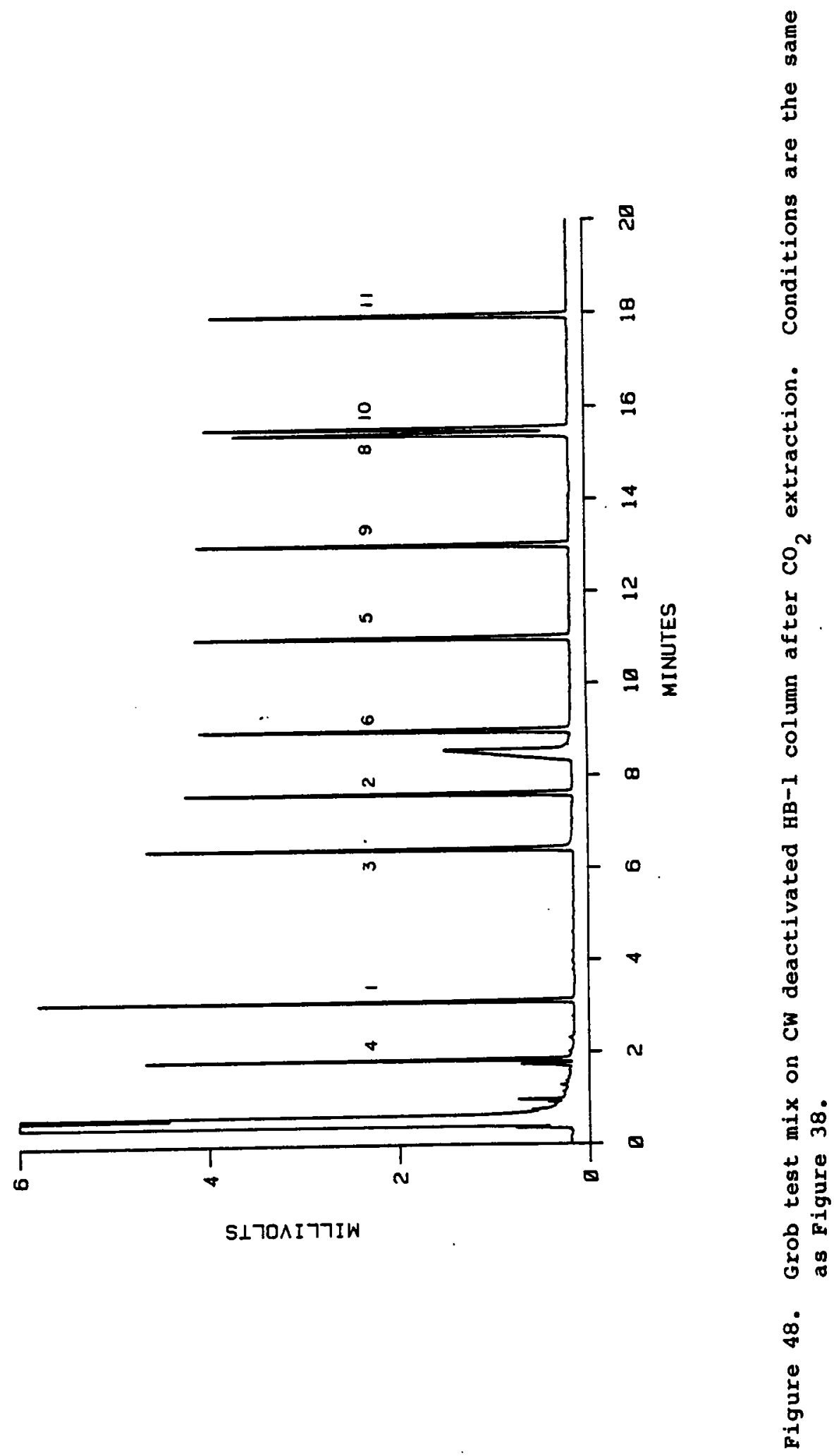


columns coated with $\mathrm{HB}-1$ were evaluated by comparing the Kovat's Retention Index $(I)^{220}$ of the first 5 McReynold's probes. ${ }^{22}$ one column was deactivated with Carbowax $20 \mathrm{M}$ while the other was coated on an untreated fused silica column. The retention index is obtained by measuring the retention time of the probe relative to two adjacent nalkanes using the following relationship:

$$
I(x)=100 z+100 \frac{\log t^{\prime}(x)-\log t^{\prime}(z)}{\log t_{R}^{\prime}(z+1)-\log t^{\prime}{ }_{R}(z)}
$$

where $x$ is the probe molecule

$z$ is the lower n-alkane

$z+1$ is the higher alkane

and $t^{\prime}{ }_{R}$ is the relative retention time

From Table 15 it can be seen that the Carbowax deactivation layer is slightly more polar than the untreated surface, as indicated by the higher I values. However, the difference is very slight (less than 0.38 ) and is unlikely to affect the separation. A more remarkable difference is seen when comparing the reproducibility as shown by the standard deviations. For polar samples, deactivation increases retention time reproducibility.

\section{Reproducibility Aspects of Capillary SFC}

Quantitative studies of capillary SFC have not been addressed in the literature until very recently. ${ }^{131}$ The emergence of SFC as a useful analytical tool is dependent on the ability of the technique to reproducibly elute various samples. Fortunately, the use of HPLC type 
Table 15. Data for Kovat's Retention Index of 5 Polar Compounds on $\mathrm{HB}-1$ As a Function of Deactivation

$(N=4)$. Column temperature $35^{\circ} \mathrm{C}$

Rovat's Retention Index $(n=5)$

Compound

Benzene

Butano1

Pentanone

Nitropropane

Pyridine
Undeactivated

$744.1 \pm 0.8$

$745.1 \pm 1.3$

$776.6 \pm 0.6$

$871.3 \pm 1.1$

$866.9 \pm 2.6$
CW Deactivated

$744.1 \pm 0.6$

$748.9 \pm 0.7$

$778.0 \pm 0.7$

$874.4 \pm 0.4$

$864.7 \pm 0.1$ 
loop injectors operated at room temperature provides the potential for a reproducible injection system. The fixed volume loop can be exhaustively flushed with sample and can be expected to deliver more reproducible injection volumes than syringe injection would provide. In addition, SFC inlet splitters can be operated at low enough temperatures so that splitting will not be affected by the differential vaporization of samples containing components widely differing in volatility. In SFC, splitting occurs in a dense, liquid like medium rather than in a hot vapor, therefore, the potential of SFC as a reproducible, nondiscriminating injection system for thermally labile compounds should approach that of cold on-column injection in GC.

When evaluating a chromatographic system for quantitative reproducibility, a major influencing factor is the interaction of the sample with the column. When only the column is changed from run to run, changes in chromatographic reproducibility can be related to the column's influence.

The effect of column deactivation in SFC has not been adequately explored. Although some effects are expected, the magnitude of the effects was a matter of some conjecture at the beginning of this study. Compared to GC, SFC normally operates at a lower temperature. Since adsorption is temperature dependent, it could be argued that deactivation is more critical in SFC than in GC. However, the solubility of the analytes in the mobile phase could decrease the degree of sample adsorption. Although adsorption in HPLC is a problem, it is most often related to the use of high surface area silica gel particles 
as a column support. With the lower surface area and greater inertness of fused silica, the effects of adsorption should not be as pronounced. The initial studies of column activity and crosslinking showed that deactivation layers of a pyrolyzed polyethylene glycol were stable and did give good deactivation when the column was used for GC. The use of ATB as a crosslinking initiator was found to be suitable for SFC use. These factors were incorporated into a study of deactivation effects during chromatography in a supercritical fluid mode.

\section{Reproducibility of SFC}

Several improvements to the preparation of the columns were made. Superox 20M was substituted for Carbowax $20 \mathrm{M}$ as the deactivation reagent. Both materials are polyethylene glycols, but the superox 20M has a greater purity and a narrower molecular weight range. In addition to this deactivation method, another deactivation method was evaluated. The use of cyclic siloxanes (primarily $D_{4}$ ) has been shown to provide good surface deactivation. 194 The use of $D_{4}$ produces a low energy methylated surface, but other cyclics containing different pendant functional groups are now coming into use. 195 It was decided that a deactivation mixture corresponding in percentage of various functional groups to the stationary phase (ov-1701) would be used. This deactivation layer would then be very similar to the stationary phase and should be wettable.

One improvement involved the incorporation of a column cleaning and activation procedure developed by Ogden and McNair. ${ }^{169}$ This 
hydrothermal treatment with $\mathrm{HNO}_{3}$ has been shown to increase the hydroxylation of the fused silica surface. The increased number of hydroxyl groups provide active sites for the deactivation layer to attach and result in improved surface coverage and more complete deactivation.

The relative standard deviation of peak areas was calculated for each component on each of the three columns. Figure 49 shows a chromatogram of the paraffin mixture while Table 16 summarizes the results of the alkanes on the three columns. The summarized results show that the average standard deviations for alkanes as a class are very similar for all three columns. This, combined with the observed symmetrical peak shapes show that column connections or dead volumes are not exerting an undue influence on any of the columns.

The alcohol test mixture is more interesting. Although in Figure 50 the peaks appear symmetrical, electronic integration shows that differences in the amount of component eluted is a function of column deactivation. Table 17 presents the results for the alcohols. From this information, it can be seen that deactivation improves the reproducibility of alcohol quantitation and the superox $20 \mathrm{M}$ deactivation exhibits the least variation in peak areas confirming that it is the most deactivated column.

The largest differences in peak area reproducibility is seen with the fatty acid sample. The chromatogram of the acids on the undeactivated column shows severe adsorption of the later eluting acids and obvious decreases in peak area (Figure 51). The results of this 


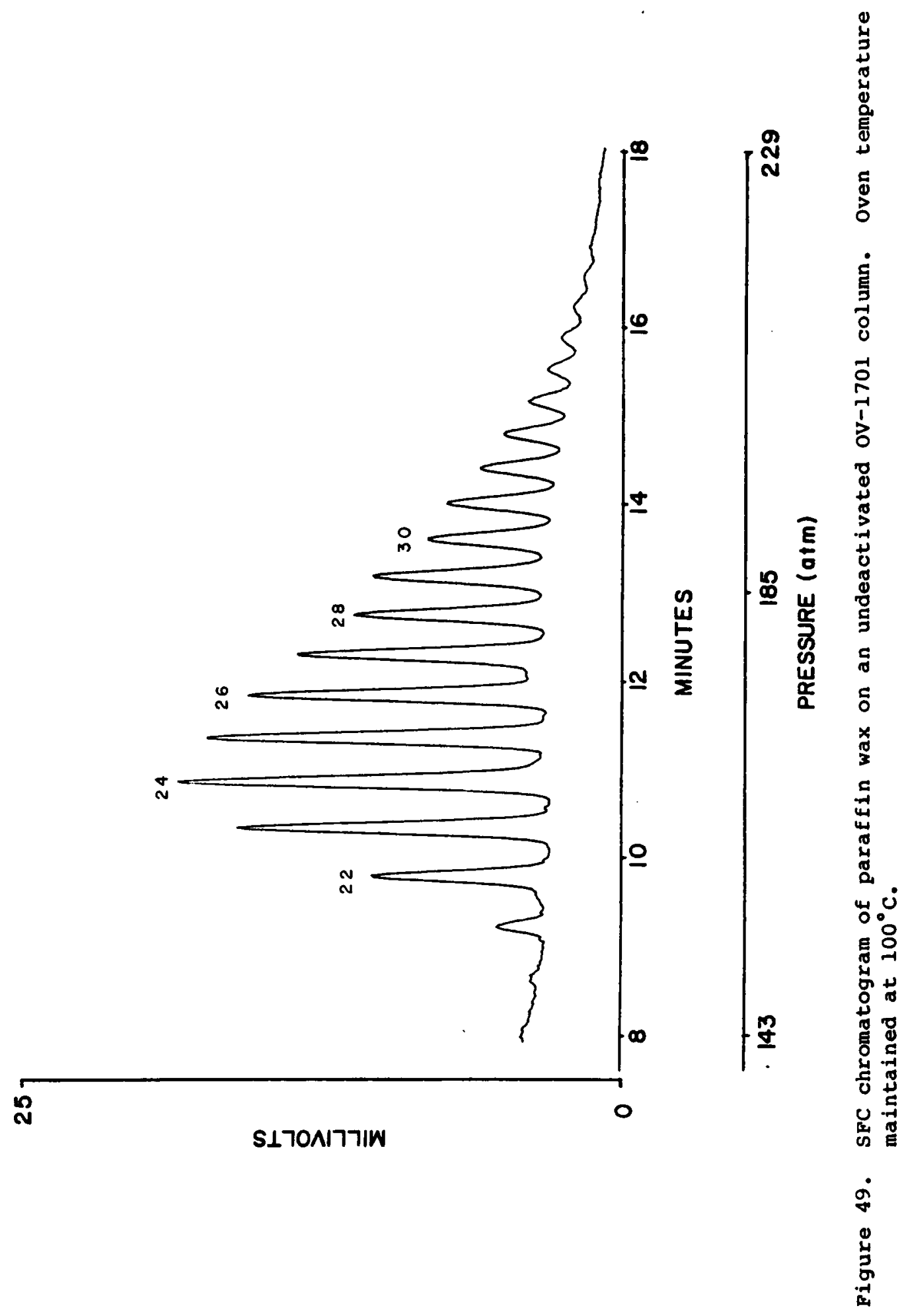




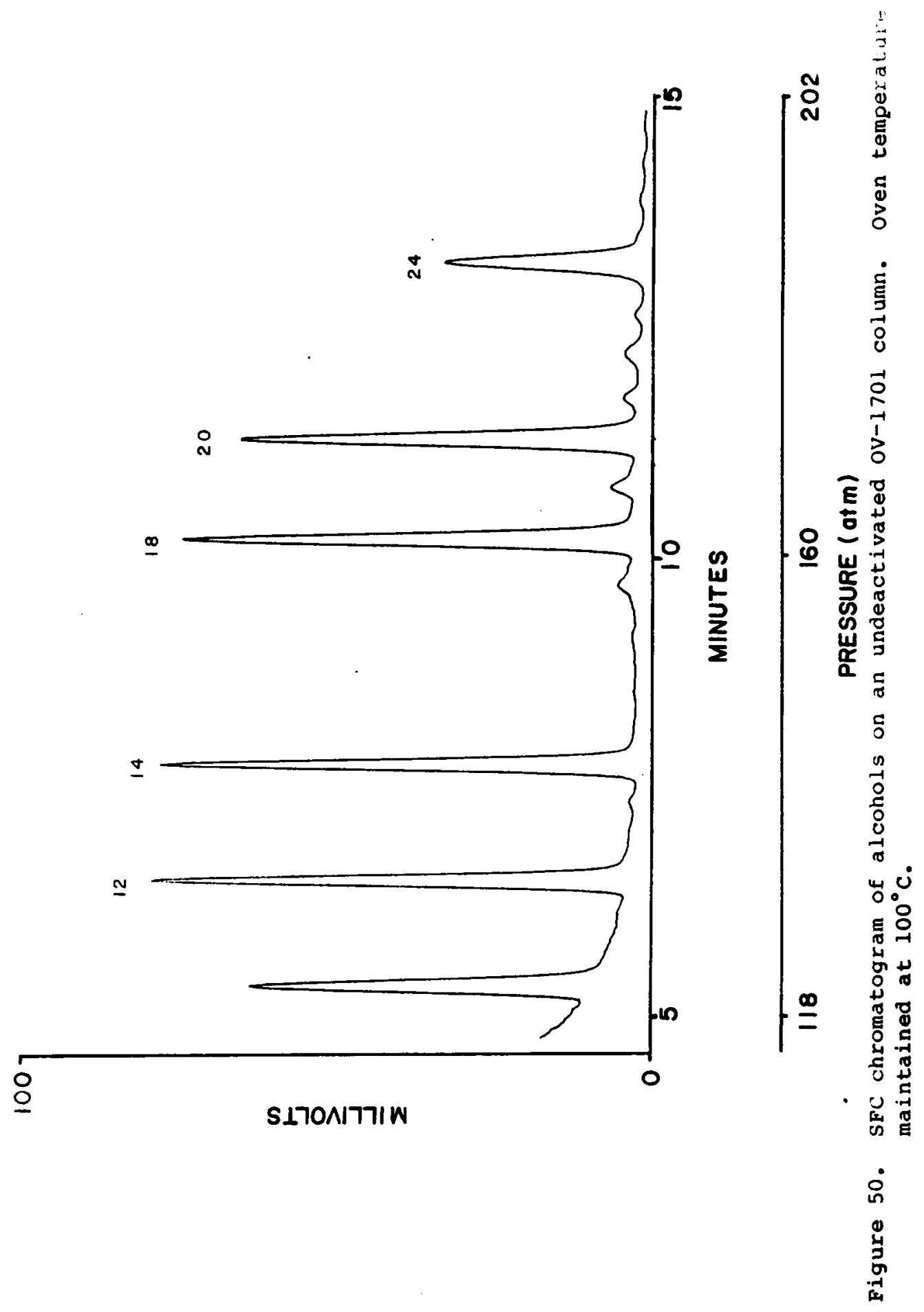


171

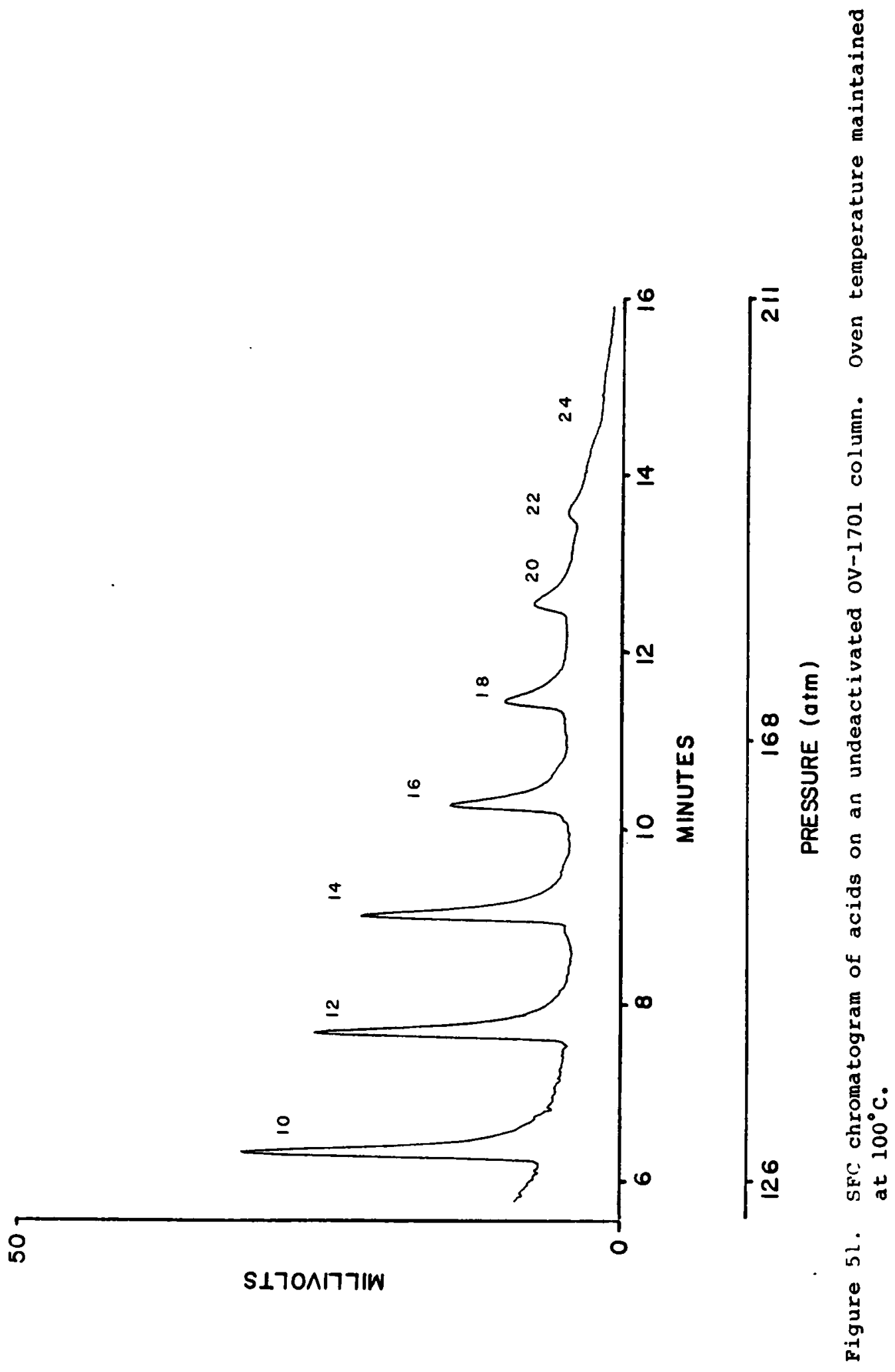


Table 16. Relative Standard Deviations of Peak Areas for Paraffins

\begin{tabular}{|c|c|c|c|}
\hline Carbon \# & $\begin{array}{l}\text { Undeactivated } \\
\text { Column }\end{array}$ & $\begin{array}{l}\text { Mixed Cyclic } \\
\text { Deactivated } \\
\text { Column }\end{array}$ & $\begin{array}{l}\text { Superox } 20 \mathrm{M} \\
\text { Deactivated } \\
\text { Column }\end{array}$ \\
\hline 22 & 4.5 & 5.5 & 2.2 \\
\hline 23 & 1.6 & 3.3 & 1.3 \\
\hline 24 & 2.9 & 2.6 & 1.4 \\
\hline 25 & 3.8 & 4.2 & 1.0 \\
\hline 26 & 7.4 & 4.3 & 1.6 \\
\hline 27 & 10.1 & 3.4 & 3.5 \\
\hline 28 & 7.7 & 8.0 & 5.3 \\
\hline 29 & 5.6 & 8.0 & 7.2 \\
\hline 30 & 4.6 & 7.2 & 10.5 \\
\hline 31 & 4.0 & 9.4 & 14.8 \\
\hline$\underline{32}$ & 4.7 & $\underline{6.7}$ & 10.6 \\
\hline $\begin{array}{l}\text { Mean RSD } \\
\text { for paraffins } \\
\text { as a chemical } \\
\text { class }\end{array}$ & 5.28 & 5.78 & 5.48 \\
\hline
\end{tabular}


Table 17. Relative Standard Deviations of Peak Areas of Alcohols

\section{Carbon \#}

12

14

18

20

$\underline{24}$

Mean RSD

for alcohols

as a chemical

class

\author{
Mixed Cyclic \\ Deactivated \\ Colunn
}

$\begin{array}{ll}\text { Undeactivated } & \text { Mixed Cyclic } \\ \text { Column } & \text { Deactivated } \\ & \text { Column }\end{array}$

3.9

3.2

2.9

3.3

$\underline{5.1}$

3.78

\section{Superox 20M \\ Deactivated \\ Column}

1.1

1.0

0.9

2.3

1.1

1.38 
Table 18. Relative Standard Deviations of Peak Areas of Saturated Fatty Acids

\begin{tabular}{|c|c|c|c|}
\hline Carbon & $\begin{array}{l}\text { Undeactivated } \\
\text { Column }\end{array}$ & $\begin{array}{l}\text { Mixed Cyclic } \\
\text { Deactivated } \\
\text { Column }\end{array}$ & $\begin{array}{c}\text { Superox } 20 \mathrm{M} \\
\text { Deactivated } \\
\text { Column }\end{array}$ \\
\hline 10 & 15.6 & 4.7 & 2.5 \\
\hline 12 & 13.4 & 7.8 & 0.9 \\
\hline 14 & 18.9 & 5.2 & 0.6 \\
\hline 16 & 36.7 & 8.6 & 1.2 \\
\hline 18 & 36.9 & 5.1 & 2.2 \\
\hline$\underline{20}$ & 47.3 & $\underline{2.6}$ & $\underline{4.4}$ \\
\hline $\begin{array}{l}\text { Mean RSD } \\
\text { of acids as a } \\
\text { chemical class }\end{array}$ & 28.18 & 5.78 & 2.08 \\
\hline
\end{tabular}


adsorption of analyte are seen in Table 18 which shows a relative standard deviation of over 28 for acids as a chemical class. Results are presented only for the c-10 through $c-20$ acids since the $c-22$ and C24 acids were absorbed so strongly that integration could not be obtained.

The Superox $20 \mathrm{M}$ deactivation provided the most reproducible results (2.08), but, as seen in Figure 52, tailing of the peaks on the polyethylene glycol deactivated surface was still observed.

The free acids chromatographed best on the cyclic deactivated column if only peak shape is taken into account (Figure 53). However, the improved peak shape does not correspond to an increase in peak reproducibility.

Several observations can be drawn from these data. It can be seen that there is a rapid increase in adsorption for the higher acid homologs relative to the lower ones. This can be attributed to a longer residence on the column which culminates in a greater period of interaction between the column and the analyte. In regard to the observed contradictions in proper peak shape but better reproducibility the following factors should be considered. Two types of adsorption processes are encountered in chromatography. These are classified as reversible and irreversible adsorption. Reversible adsorption is characterized by peak tailing and is considered to be a weak interaction of adsorptive sites on the column with active sample components. Irreversible adsorption is a stronger interaction which usually results in a binding of the analyte to the column. This interaction can reduce 


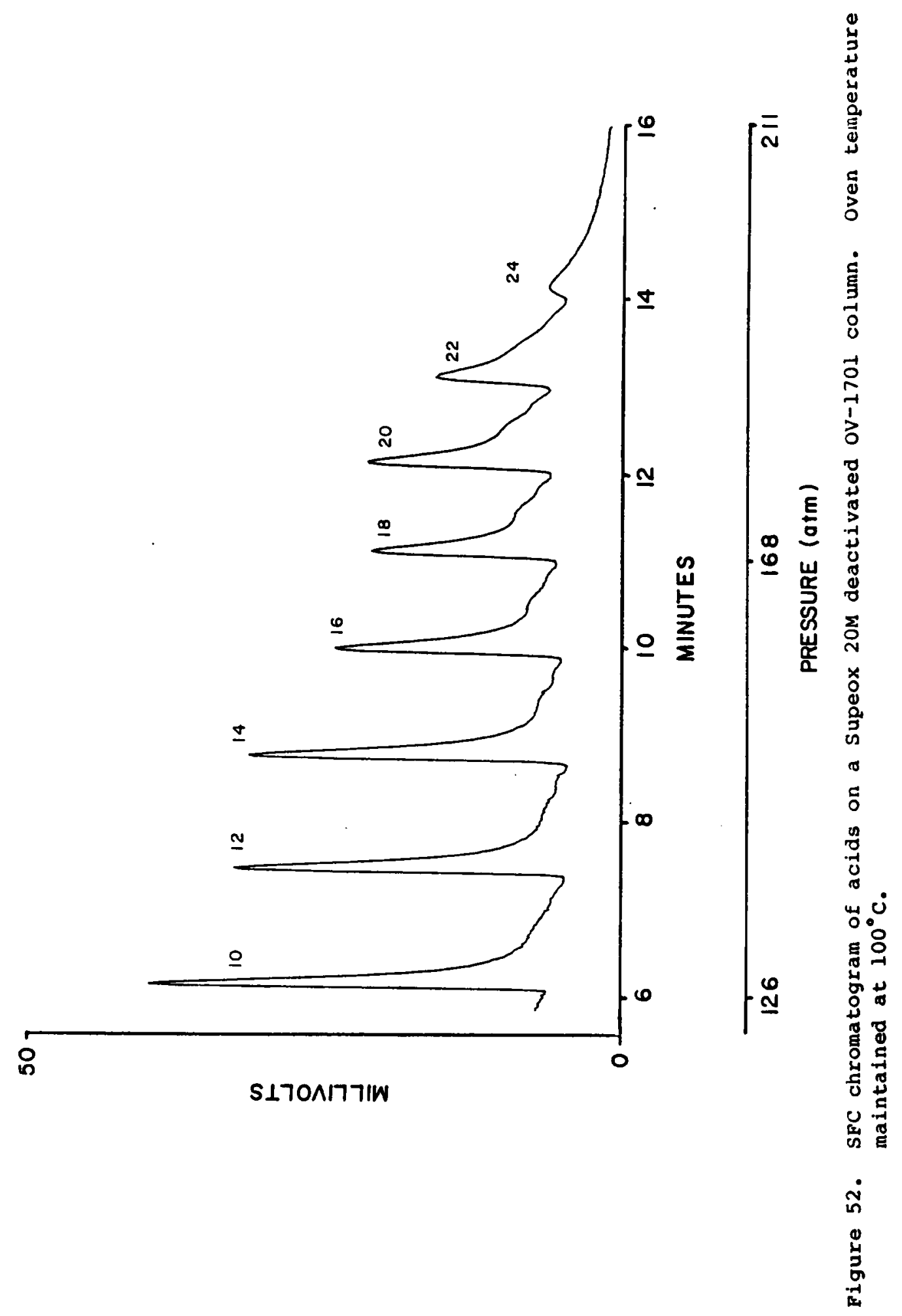




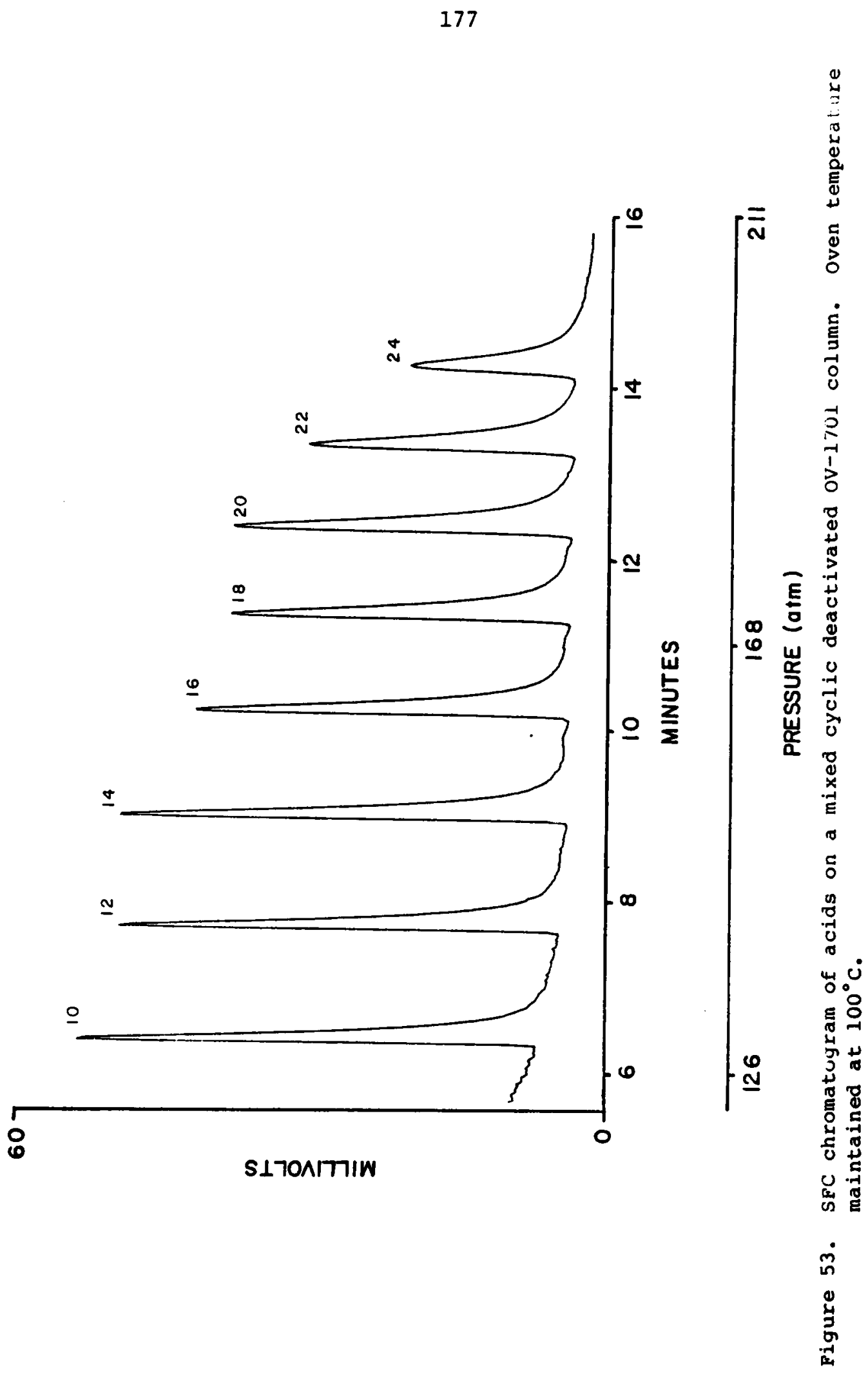


the area of a peak or cause it to disappear entirely. The results that are obtained with these different deactivation methods would appear to be related to differences in the adsorption processes occurring. The Superox $20 \mathrm{M}$ column would appear to have reversible adsorption which occurs in a reproducible manner. This causes peak tailing but the amount of material eluted remains relatively constant. The column deactivated with the cyclic mixture shows little reversible adsorption but the decreased reproducibility could be related to irreversible adsorption. Irreversible adsorption has the potential to give less reproducible results than reversible adsorption since active sites are being covered with each injection of analyte. The removal of adsorbed material is a slow process if it is possible for it to even be removed. With reversible adsorption, the material is eluted for the next analysis. Although both types of adsorption are detrimental to reproducibility, the degree of harm is different. 
CHAPTER VII

CONCLUSIONS

\section{Instrumentation}

A chromatograph using supercritical fluid $\mathrm{CO}_{2}$ as a mobile phase can be modified using a standard gas chromatograph with the addition of a pulse free source of high pressure $\mathrm{CO}_{2}$ and a small volume high pressure sampling valve. It has been shown that limiting the amount of material injected onto the SFC column is crucial for resolution of sample components to be maintained. A $0.1 \mu \mathrm{l}$ sample loop allows the direct injection of sample onto the column while a $0.5 \mu 1$ loop requires that the sample volume injected be reduced by the addition of an inlet splitter.

The flame ionization detector was found to work well for samples separated by SFC. A simple, linear, $10 \mu \mathrm{m}$ i.d. restrictor maintained the column pressure while transmitting a reasonable flow rate to the detector. The problems reported by other workers with noise spikes in the detector were rarely observed with this system. It is probable that the problems reported by other workers are an artifact of the geometry of the detector design. The FID in the Perkin-Elmer 2000 performed well and seldom exhibited noise spikes during a run.

\section{Injection Technique}

Practical application of the concept of a solubility threshold in SFC was demonstrated for the first time. This technique, which is analogous to cold trapping in GC, permits the on-column concentration of 
180

analyte by repetitive injection of the sample at conditions where sample migration does not occur. This technique allows lower concentrations of compounds to be analyzed than would be possible with a single injection. For this injection technique to be effective, injection must occur at a density low enough not to dissolve the compound and a temperature at which the compound is not volatile enough to migrate.

\section{Polyethylene Glycol Deactivation}

The results of testing GC columns under SFC conditions was successful in showing the effect of supercritical fluid $\mathrm{CO}_{2}$ on column activity and efficiency. The chemistry and procedures used in making capillary columns are nearly identical, and the increased capacity of the larger columns allows evaluation to be made using standard methods borrowed from capillary GC.

Deactivation with polyethylene glycol (Carbowax) pyrolysis products was found to provide an inert surface which was non-extractable and did not lead to phase stripping by $\mathrm{CO}_{2}$ or to droplet formation during coating of semi-polar phases. The temperature limitations of Carbowax deactivation in GC $\left(200^{\circ} \mathrm{C}\right)$ do not apply to SFC columns since temperatures over $200^{\circ} \mathrm{C}$ would rarely be used. The advantages of a good deactivation layer which provides a high energy surface ( 45 dynes/cm) for coating are therefore made available for capillary SFC.

Crosslinking with dicumyl peroxide (DCP) is sufficient for easily crosslinked materials, particularly if vinyl groups have been incorporated, however, the effects of the decomposition products of DCP 
181

at the higher concentrations needed to crosslink more polar phases show increased a negative impact on column activity. It has been shown by SFC extraction that even at a 48 loading, DCP is unable to completely crosslink ov-1701 when extraction with supercritical fluid $\mathrm{CO}_{2}$ is performed.

For ov-1701, azo-tert-butane (ATB) was found to crosslink the phase completely, and the lack of polar decomposition products produced a column which was inert to adsorption and was neutral in $\mathrm{pH}$. This held true even after repeated extraction with $\mathrm{CO}_{2}$ under supercritical fluid conditions for many hours.

A crosslinked commercial methyl silicone column was evaluated under the same conditions as above. While little is reported by manufacturers on details of column manufacture and crosslinking, the results obtained are consistent with peroxide use as a crosslinking initiator. The lack of complete crosslinking in this 1008 methyl polysiloxane phase indicates that commercial vendors of columns for SFC should increase the effectiveness of their crosslinking compared to that needed for GC.

\section{Reproducibility of SFC}

Deactivation of capillary columns for SFC was found to be the crucial factor in obtaining reproducible quantitative results. The results show that the ability of $\mathrm{SFC}$ to handle polar analytes in a reproducibile manner is strongly dependent on column deactivation. This is important since the major advantage of SFC is the ability to handle thermally labile compounds which usually are labile due to the presence of polar functional groups. The importance of column deactivation is 
182

directly proportional to the polarity of the sample. For paraffins and other non-polar samples, no need for deactivation is seen. In the case of the strongly polar acids, deactivation is critical for good results. Deactivation with the superox $20 \mathrm{M}$ results in the most reproducible results indicating that the surface coverage of the deactivation is more complete. The cyclic mixture shows less reversible adsorption but irreversible adsorption by active sites is encountered. The ability to cover these sites is limited since increased time for deactivation at the high temperature needed to open the cyclic siloxane ring results in deterioration of column handling properties due to the degradation of the exterior polyimide coating.

Deactivation by polyethylene glycol pyrolysis combines a low decomposition temperature with a resulting high energy surface to yield a deactivation method suitable for SFC. The disadvantages of polyethylene glycol pyrolysis which result in high column bleeding at high temperatures are not a problem under the milder temperature conditions of SFC.

\section{Future Work}

Capillary SFC is a new technique and advances need to be made in many areas. The coating of capillary columns with uniform films of crosslinked, polar, stationary phases should be developed for SFC due to the added selectivity which would result from use of these columns. As this work demonstrates, concurrent advances in column deactivation should also be sought. 
183

The instrumentation for SFC is still crude. It can be assumed that extended use will result in new designs which will optimize performance. This is particularly indicated in the case of detector design. The FID was designed as a GC detector and it would be very surprising if it were optimized for SFC in terms of sensitivity and linear range. 
AP PENDIX A

Schematic of circuitry for modification of ISCO $\mu L C 500$ to permit linear pressure programming.

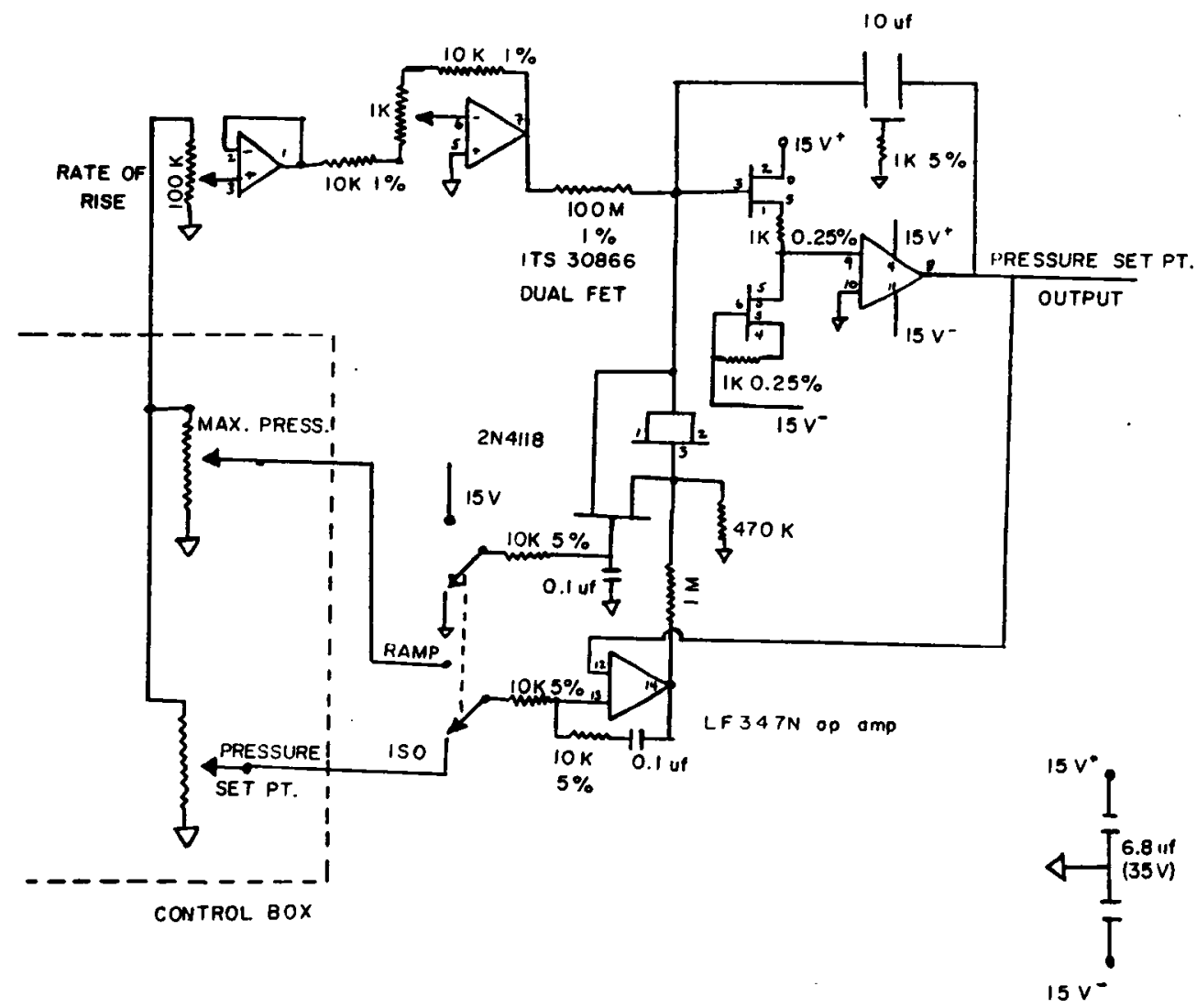


APPENDIX B

\section{Applications}

\section{Introduction}

Many samples are amenable to analysis by SFC. However, the ability to separate components by SFC is not in itself a justification for SFC. If a sample is capable of withstanding the thermal stress of gas chromatographic analysis, then GC is the favored method from both theoretical and practical considerations. The application of SFC is best demonstrated on samples that are non-volatile or thermally labile.

\section{Practical Operation}

Practical sample analysis is affected by several factors. The sample size must be sufficient to allow for reliable detection and quantiation, the linear range of the technique should be fairly large so samples can be analyzed without excessive time spent in dilution and concentration. The $100 \mathrm{\mu m}$ i.d. columns have been shown to have good linear range and reasonable sample loading. Smaller, 50 um i.d.. columns have been evaluated but they have a smaller sample capacity which results in a need to decrease loading significantly.

Practical utilization of an analytical technique is also dependent on analysis time. Unfortunately, the optimal linear velocities for capillary SFC are very slow. It has been calculated that a velocity of $0.12 \mathrm{~cm} / \mathrm{sec}$ would be optimal for a $100 \mu \mathrm{m}$ i.d. capillary column. 31 A slow velocity gives long analysis times. For a 10 meter column, this optimal velocity would take 2.3 hours to elute an unretained peak. If a sample with a $k^{\prime}$ of 3 were analyzed, an analysis time of 6.9 hours would 
be expected. Normally, the column is operated well above its optimal velocity ( $\mu_{\text {opt }}$ ) to decrease the analysis time. Operation at 10 to $20 \mathrm{x}$ $\mu_{\text {opt }}$ results in reasonable analysis time $\left(t_{0}=7\right.$ to 14 minutes for a 10 meter column. The effect of operation at higher linear velocities is a reduction in column efficiency due to non-equilibrium due to mass transfer in the mobile and stationary phase.

An experimental determination of the height equivalent to a theoretical plate (HETP) vs. linear velocity was performed at 2500 psi and $150^{\circ} \mathrm{C}$ using $\mathrm{CO}_{2}$ as the mobile phase on a $100 \mu \mathrm{m}$ i.d. column. Tetracosane was used as the sample probe $\left(k^{\prime}=0.7\right)$. It can be seen that an increase in linear velocity leads to an increased plate height and a decreased efficiency (Figure 54). In the interests of a reasonable analysis time, the majority of application work was performed at $2 \mathrm{~cm} / \mathrm{sec}$ linear velocity.

\section{Selected Applications}

Some typical SFC applications have been encountered in earlier chapters. The separation of paraffins and the dimethyl polysiloxane oligomers demonstrate the suitability of SFC to higher molecular weight samples. The non-ionic surfactant Triton $x-100$ can also be analyzed easily by SFC. This is an example of a material lacking sufficient volatility for gas chromatographic analysis. Figure 55 shows a typical analysis of Triton $x-100$. Note that the resolution is sufficient to show the small impurities occurring at the base of the major peaks. The ability of supercritical fluids to dissolve some polymeric 


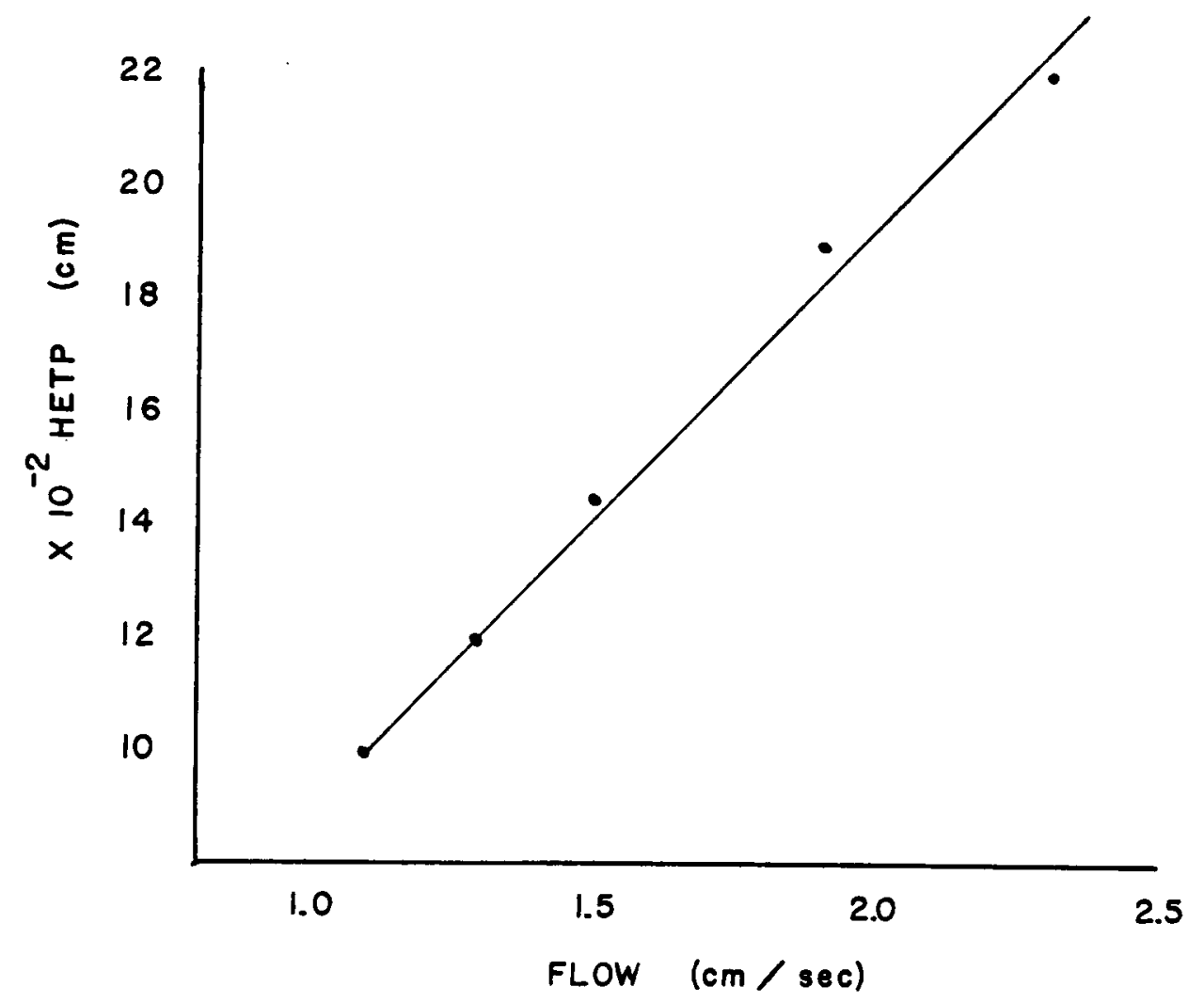

Figure 54. The effect of fast column flow rates on the height of the effective theoretical plate (HETP). 


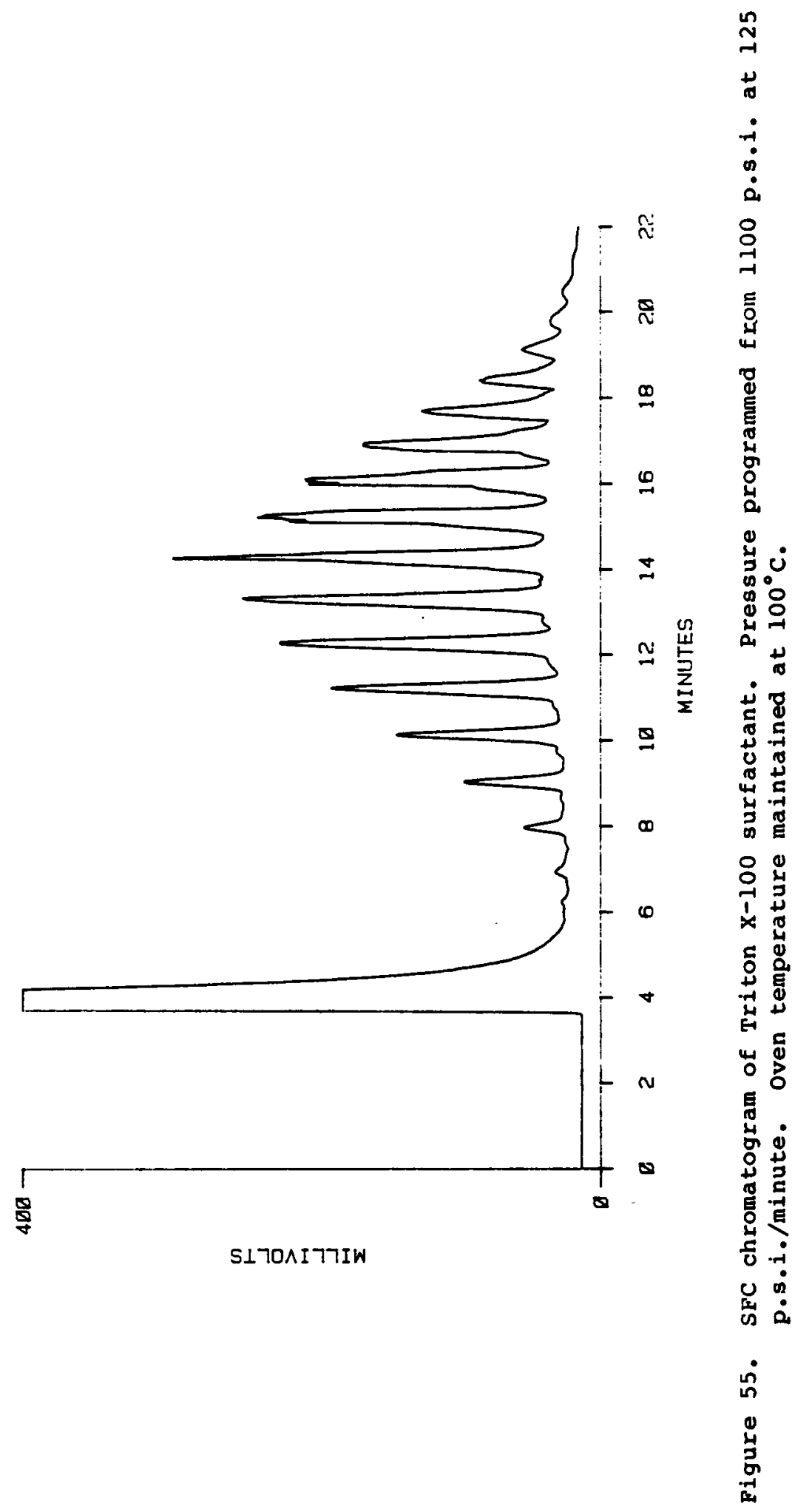


materials has suggested its use for polymer characterization. The use of the FID provides a sensitive method for detecting compounds which might lack a UV chromophore or be present in amounts too small for detection using refractive index detection. Figure 56 shows another dimethylpolysiloxane, SF-96, which is sometimes used as a stationary phase for gas chromatography. The profile is suggestive of two distinct forms which could possibly be a linear siloxane in combination with a branched or cyclic siloxane.

A second polymer $(O V-235)$ was also evaluated. This is an experimental GC liquid phase which has an approximate molecular weight of 6000 Daltons. The polymer is a cyanopropylphenylmethylpolysiloxane with some vinyl incorporated for ease in crosslinking. The polymer was not uniform in appearance with both a gum and a liquid phase existing in the stock bottle. When attempts at column coating failed, the polymer was analyzed by GPC with UV detection. The GPC showed two peaks indicating that two molecular weight fractions were present which incorporate UV chromophores. When the sample was analyzed by SFC/FID, three peaks were detected (Figure 57). The third peak is presumably a Eraction which lacked phenyl groups. This shows that the polymer is not homogenous, and uniform coating should not be expected.

\section{Analysis of Flavors, Fragrances, and Spices}

One of the major areas of application of SFC may be the analysis of flavors, fragrances, and spices. These components are in many cases present at low levels and decompose thermally. These two attributes are both handled well by SFC. With complex samples, sample preparation 


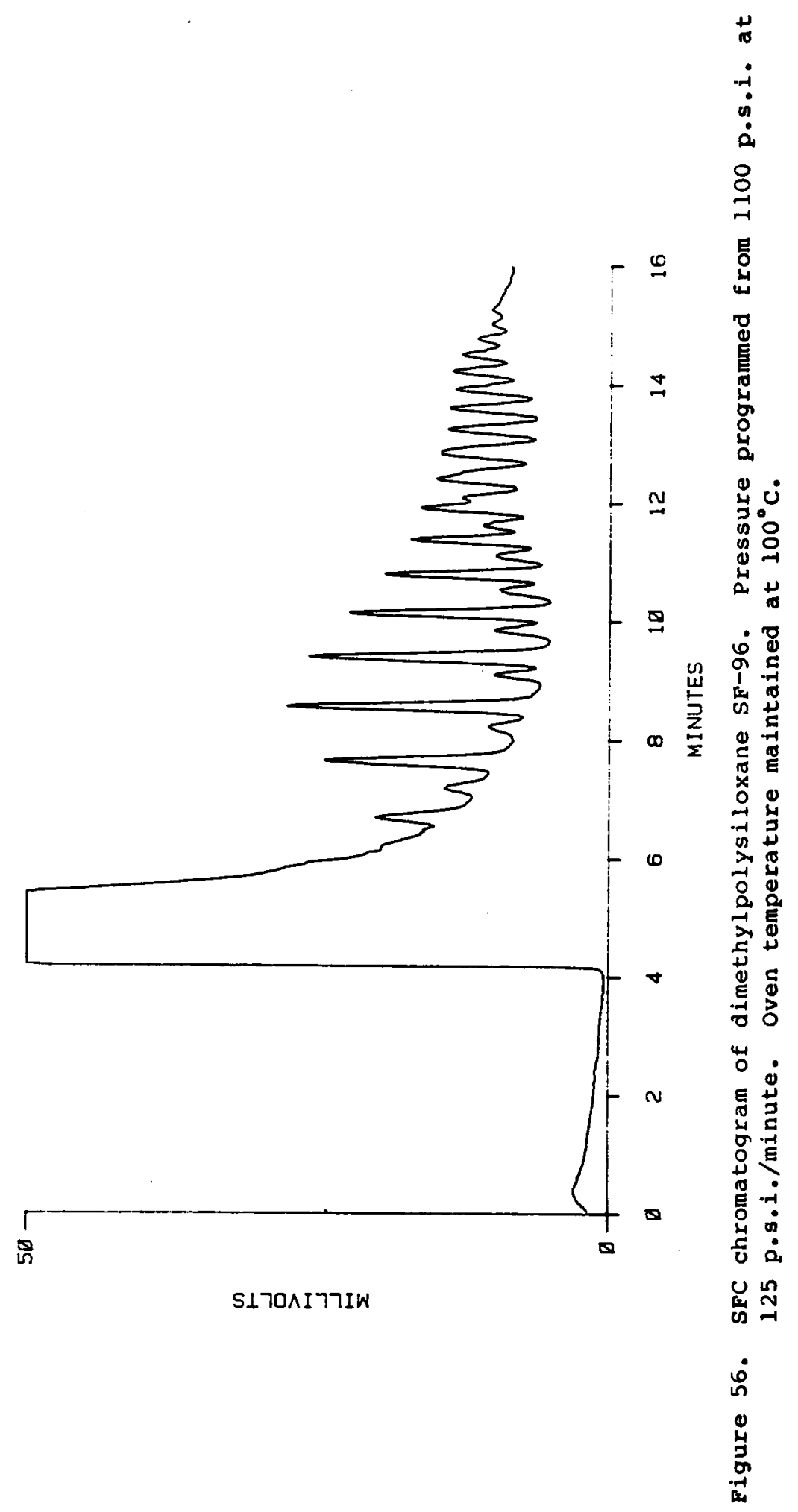




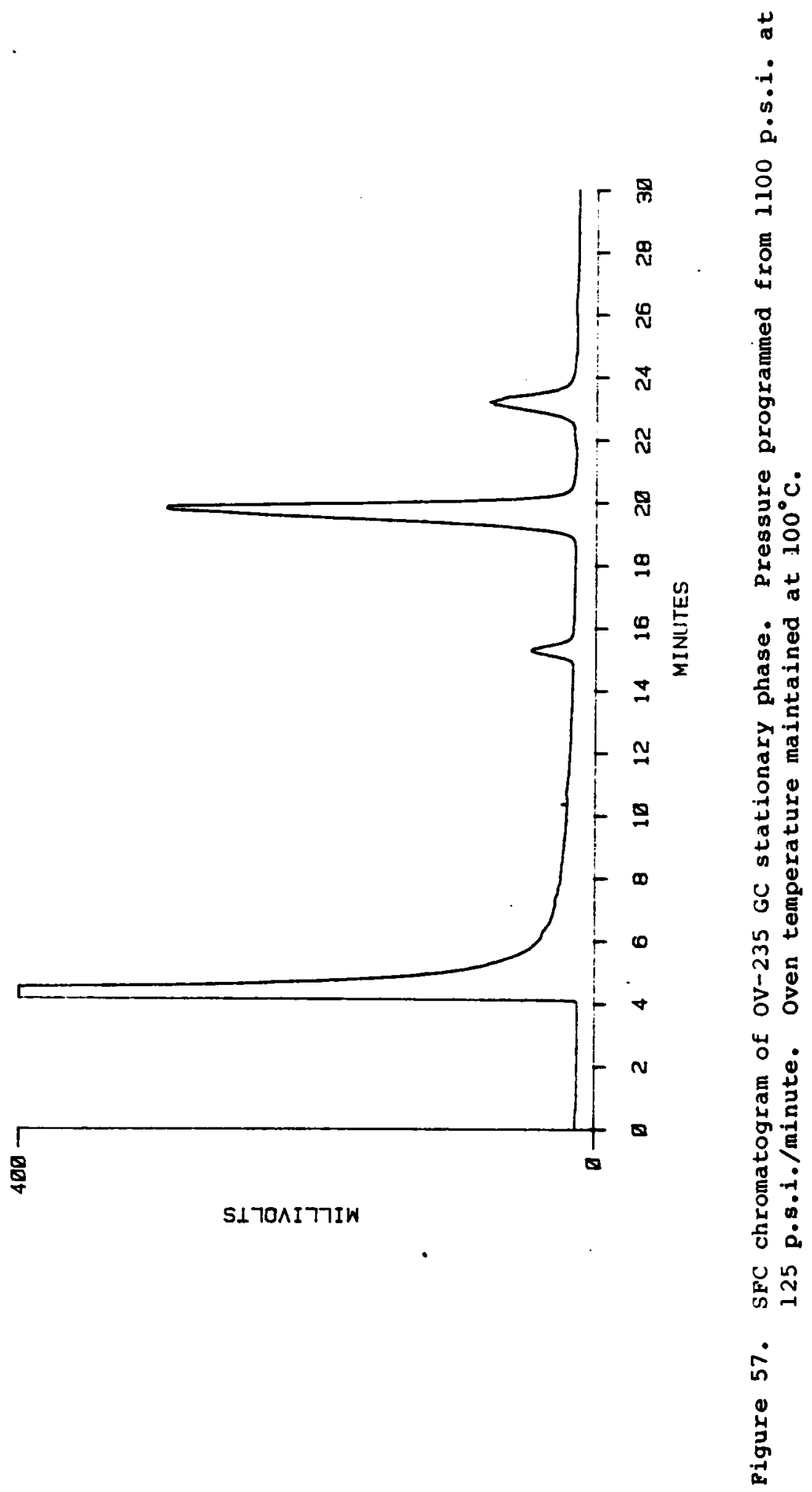


becomes increasingly important. It is necessary that the components of interest be isolated by removal from the surrounding matrix. The sample should then be prepared so that all of the injected material is soluble in the mobile phase to prevet deposition of insoluble material which could lead to column contamination and plugging.

\section{Extraction with Liquid $\mathrm{CO}_{2}$}

Extraction of material with liquid $\mathrm{CO}_{2}$ has proven to satisfy the goals of sample preparation. The sample is removed from the matrix by the extraction process, and since $\mathrm{CO}_{2}$ is the extraction solvent, the material is of course soluble in the supercritical $\mathrm{CO}_{2}$.

The extraction of the components of interest is easily checked, when dealing with spices and fragrances, by smelling the extraction residue. The $\mathrm{CO}_{2}$ is odorless and escapes as a gas at atmospheric pressure so extraction solvent removal and reconstitution in another solvent is easily accomplished.

The apparatus used was a $J \& W$ Scientific (Rancho Cordova, CA) High Pressure $\mathrm{CO}_{2}$ Soxhlet Extractor. A cellulose thimble containing the sample was placed in the glass soxhlet extractor which was then placed in the steel pressure bomb. The bottorn of the bomb was immersed in a water bath at $50^{\circ} \mathrm{C}$. Ice water at $0^{\circ} \mathrm{C}$ was circulated through the cold trap at the top to condense the $\mathrm{CO}_{2}$ as a liquid and allow it to drip through the thimble and extract the sample. The steel bomb was attached to a cylinder of $\mathrm{CO}_{2}$ equipped with a dip tube and filled with frequent venting to remove air. When $\mathrm{CO}_{2}$ "snow" appeared at the vent, venting 
ceased and the system was sealed and allowed to extract for 2 hours. The pressure in the system was $\sim 900$ psi throughout this period. When extraction was ended, the $\mathrm{CO}_{2}$ was vented and the sample was removed. The $\mathrm{CO}_{2}$ quickly sublimed after the sample was removed from the chamber and the fragrant residue was collected.

The extract was dissolved in carbon disulphide to allow injection into the chromatograph. $\mathrm{Cs}_{2}$ was used since it has a low response in the FID. The samples analyzed included bay leaves, basil, oregano and milk chocolate. These are shown in Figures 58-61. Although the identification of these components requires the use of information rich detectors like MS or FTIR which are currently unavailable in our Iaboratory, these chromatograms demonstrate the potential of SFC. 


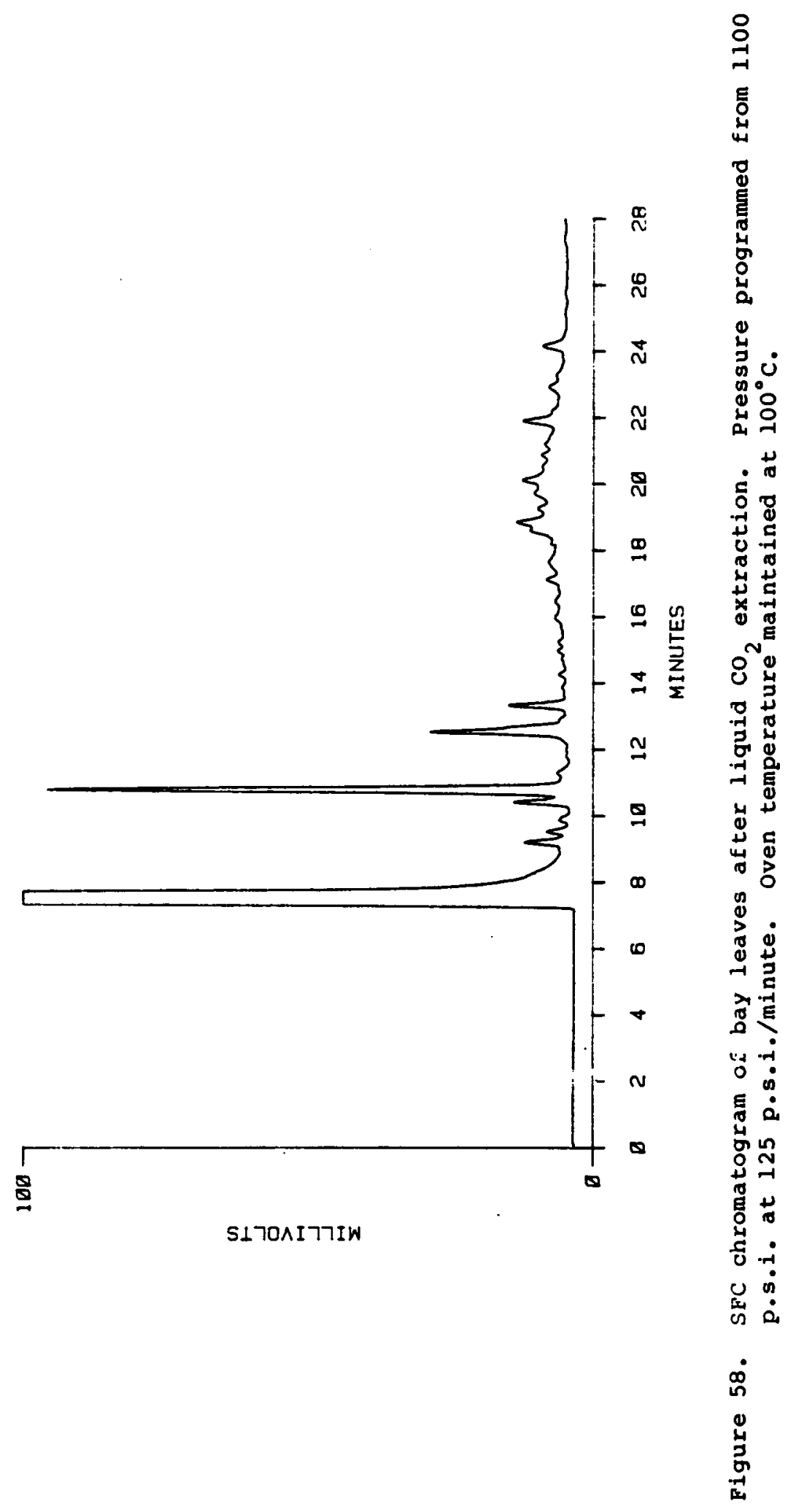




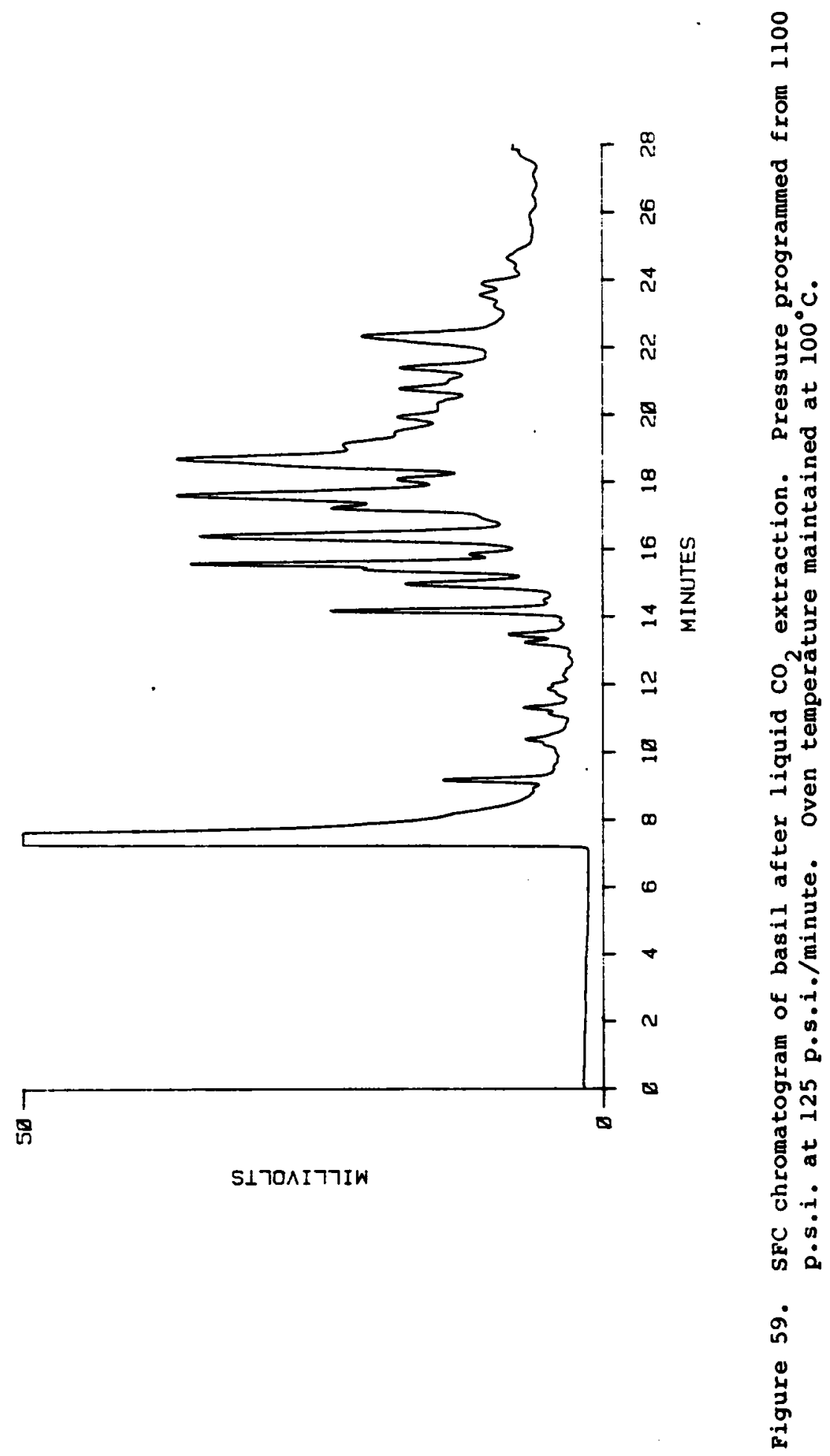




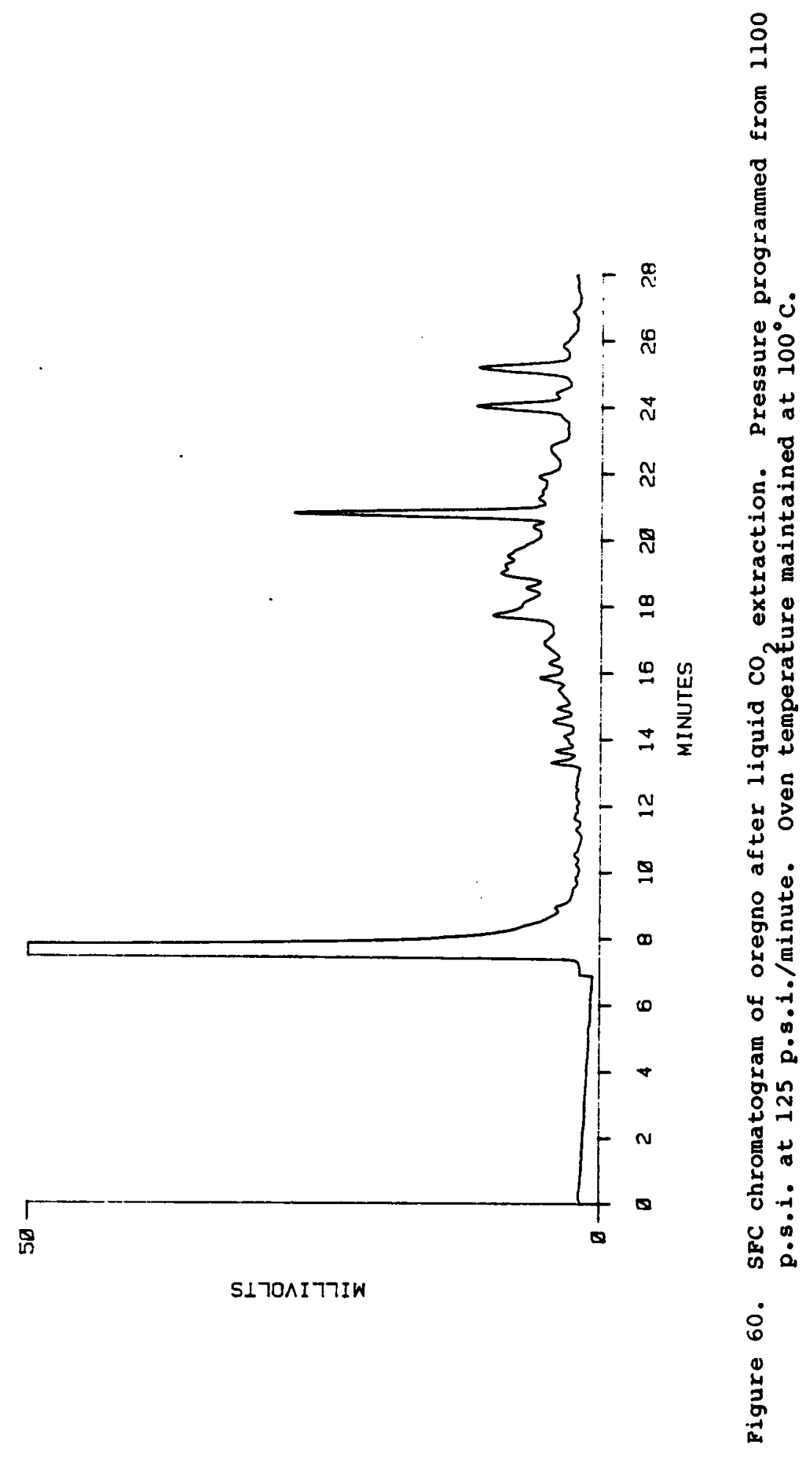




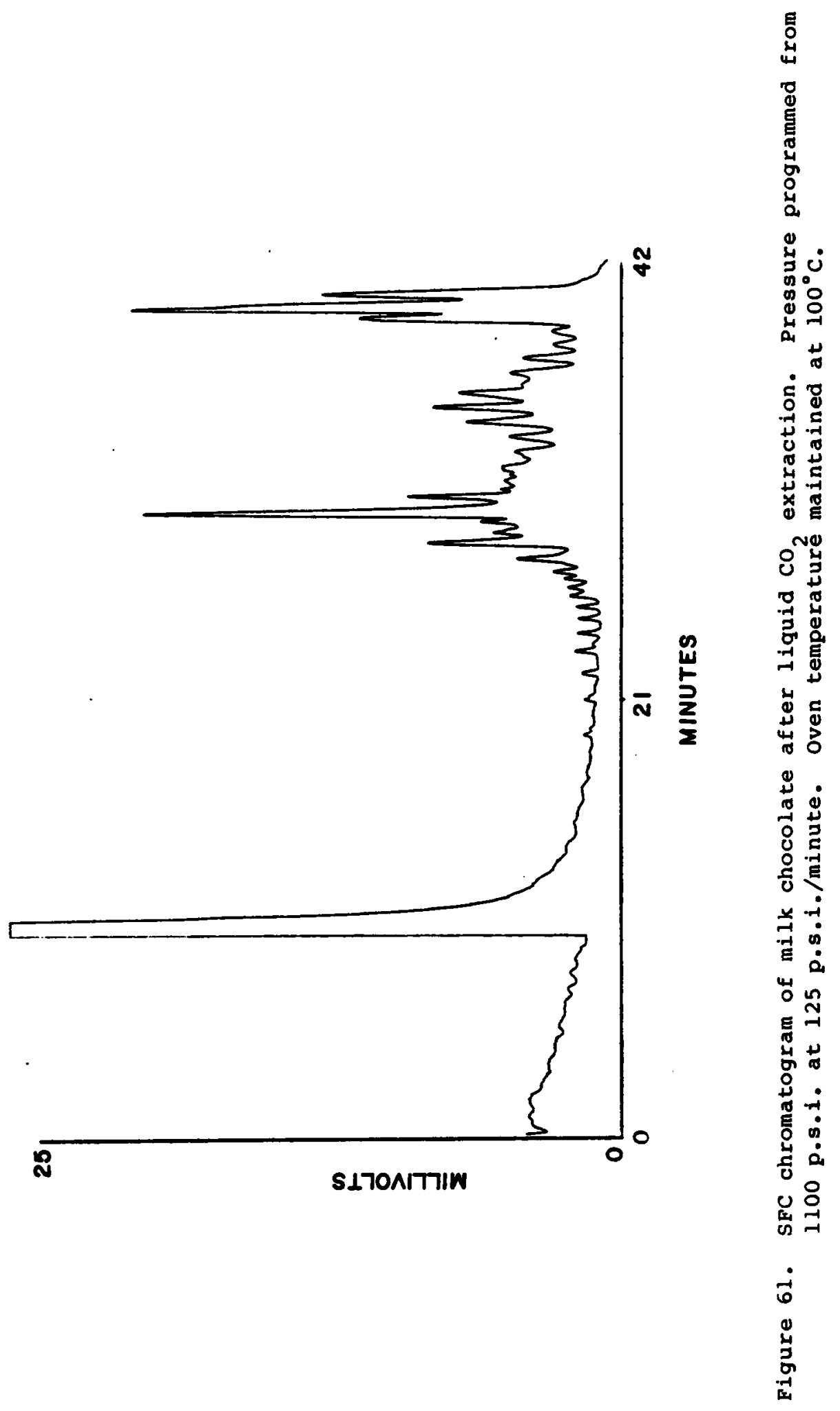




\section{REFERENCES}

1. E. Klesper, A. H. Corwin, D. A. Turner, J. Org. Chem, 27, 700 (1962).

2. M. Novotny, S. R. Springston, P. A. Peader, J. C. Feldsted and M. L. Lee, Anal. Chem. 53, 407A (1981).

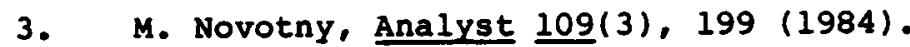

4. E. I. Johnson and R. Stevenson, Basic Liquid Chromatography, Varian, Palo Alto, CA (1978).

5. M. J. E. Golay Anal. Chem. 29928 (1957).

6. M. I. Lee, F. J. Yang and K. D. Bartle, Open Tubluar Column Gas Chromatography: Theory and Practice, Wiley-Interscience, New York (1984) 128 .

7. R. A. Alberty and F. Daniels, Physical Chemistry, J. Wiley and Sons, New York (1980) 25 .

8. M. Novotny, W. Bertsch and A. Zlatkis, J. Chromatogr., 6117 (1971).

9. M. J. E. Golay, in "Gas Chromatography 1960," R. P. W. Scott, ed., Butterworths, London (1960) 139.

10. A. S. Said, Theory of Mathematics and Chromatography, Huthig, Heidelberg (1981) 169.

11. B. L. Karger, "The Relationship of Theory to Practice in HPLC," Modern Practice of Liquid Chromatography, Ed. J. J. Kirkland, John w. Wiley and Sons, New York (1971) 21 .

12. C. M. White and R. K. Houck, HRC\& CC 9, 4 (1986).

13. G. Wilke, Angew Chem., 17, 701 (1978).

14. G. Schneider, Angew Chem 17, 716 (1978).

15. P. Hubert and O. G. Vitzthum, Angew Chem 17710 (1978).

16. Y. Adachi and B. C. Y. LU, Fluid Phase Equil 14147 (1983).

17. L. G. Randall, Technical Paper 102 Hewlett Packard Co., Avondale, PA, (1983).

18. H. H. Lauer, D. McMannigill and R. D. Board, Anal. Chem., 551370 (1983). 
19. H. Lentz and E. U. Franck, Angew. Chem. Int. Ed. 17728 (1978).

20. J. C. Fjeldsted, W. P. Jackson, P. A. Peaden and M. C. Lee, J. Chromatogr.. Sci. 21222 (1983).

21. P. Peaden, J. C. Fjeldsted, M. C. Lee, S. R. Springston and M. Nouotny, Anal. Chem., 541090 (1982).

22. H. E. Schwartz and R. G. Brownlee, Pittsburgh Conferece on Analytical Chemistry and Spectroscopy, 1986.

23. C. C. Johnson, J. W. Jordan, R, J. Skelton and L. T. Taylor, Paper 538, Pittsburgh Conference 1985.

24. S. V. Olesik, S. B. French and M. Novotny Chromatographia 18489 (1984).

25. L. M. Bowman, M. N. Myers and J. C. Giddings Sep. Sci. and Technol. 17271 (1982).

26. J. C. Giddings, M. N. Myers, L. McLaven and R. A. Reller, Science 162, 67 (1968).

27. J. B. Hannay, Proc. Roy. Soc. London 30, 484 (1880).

28. S. T. Sie and G. W. A. Rijnders Sep. Sci. 1, 459 (1966).

29. U. van Wasen and G. M. Schenider, Chromatographia $\underline{8} 274$ (1975).

30. D. Bartmann and G. M. Schneider, J. Chromatogr, 83135 (1973).

31. P. A. Peaden and M. C. Lee, J. Chromatogr.. 2591 (1983).

32. B. P. Semonian and L. B. Rogers, J Chrom. Sci. 1649 (1978).

33. S. T. Sie and G. W. A. Rijnders, Sep. Sci. 2699 (1967).

34. R. E. Jentoft and T. H. Gouw, J. Polym. Sci. Part B, 7, 811 (1969).

35. R. E. Jentoft and Gouw, J. Chrom. Sci, 8, 138 (1970).

36. D. Bartmann and G. M. Schneider, Ber Bunsenges Phys. Chem., 77, 1220 (1973).

37. J. A. Nieman and L. B. Rogers, Sep. Sci. 10, 517 (1975).

38. P. J. Schoenmakers, J. Chromatogr., 3151 (1984). 
39. B. I. Lee and M. G. Kesler, AICHE J. 21, 510 (1975).

40. P. A. Peaden and M. L. Lee, J. Lig. Chrom. 5, Supplement 2, 179 (1982).

41. J. C. Giddings, Anal. Chem. 36741 (1964).

42. M. N. Myers and J. C. Giddings, Anal. Chem. 371453 (1965).

43. J. A. Graham and L. B. Rogers, J. Chrom. Sci. 1875 (1980).

44. U. Van Wasen, I. Swaid and G. M. Schneider, Angew. Chem. Int. Ed. Eng. 19, 575 (1980).

45. J. C. Giddings, "Dynamics of Chromatography", Marcel Dekker, New York (1965) 223-224.

46. D. R. Gere, R. Board and D. MoManigill, Anal. Chem. 54736 (1982).

47. S. T. Sie, G. W. A. Rijnders, Sep. Sci. 2755 (1967).

48. B. P. Semonian, L. B. Rogers, J. Chrom. Sci. 1649 (1978).

49. J. C. Giddings, M. N. Myers, L. McLaven and R. A. Keller, Science 159197 (1968).

50. M. N. Myers, J. C. Giddings, E. S. Perry, and C. J. Van Oss Progress in Separation and Purification vol. 3, WileyInterscience, New York (1970) 133.

51. E. Klesper, Angew. Chem. Int. Ed. Eng. 17738 (1978).

52. R. Board, D. McManigill, H. Weaver and D. Gere, Paper at 1982 Pittsburgh Conference, Hewlett-Packard Publication No. 43-59531647. Hewlett Packard Co., USA (1982) 17.

53. B. W. Wright and R. D. Smith, J. Chromatogr. 355367 (1986).

54. A. W. Francis, J. Phys. Chem. 581099 (1954).

55. D. R. Dandge, J. P. Heller, and K. V. Wilson, Ind. Eng. Chem. Prod. Res. Dev. 24162 (1985).

56. J. B. Hannay and J. Hogarth, Proc. Roy. Soc. 29324 (1879).

57. W. M. Lacey, Am. Pat. Inst. Bull, 21065 (1932).

58. D. L. Katz and F. Kurata, Ind. Eng. Chem. 32817 (1940).

59. H. E. Messmore, U. S. Pat. 2420185 Philips Petrol. Co. (1943). 
60. T. P. Zhuze and A. A. Rapelyushnikov, USSR Pat. 113325 (1955).

61. T. P. Zhuze, Petroleum (London) 23298 (1960).

62. K. D. Bartle, T. G. Martin and D. F. Williams, Fuel $\underline{54} 226$ (1975).

63. K. Zosel, DBP 2005293, Studiengesellschaft Kohle (1970).

64. S. Peter and G. Brunner, Angew. Chem. Int. Ed. Eng. 17746 (1978).

65. D. Gere, Science 222253 (1983).

66. S. T. Sie and G. W. A. Rijnders, Sep. Science $\underline{2} 729$ (1967).

67. S. T. Sie and G. W. A. Rijnders, Anal. Chim. Acta. 3831 (1967).

68. R. E. Jentoft and T. H. Gouw, Anal. Chem, 44681 (1972).

69. R. E. Jentoft and T. H. Gouw, Anal. Chem. 482195 (1976).

70. T. H. Gouw and R. E. Jentoft, J. Chromatogr, 68303 (1972).

71. L. G. Randall and A. L. Wahrhaftig, Anal. Chem, 501703 (1978).

72. F. P. Schmitz and E. Klesper, Makromol. Chem. Rapid Communications 2735 (1981).

73. F. P. Schmitz and E. Klesper, Polymer Bull. (Berlin) 5603 (1981).

74. S. R. Springston and M. Novotny, Chromatographia 14679 (1981).

75. R. D. Smith, J. C. Fjeldsted, and M. C. Lee, J. Chromatogr, 247 231 (1982).

76. R. Feist and G. M. Schneider, Sep. Sci. Technol. 17261 (1982).

77. R. Board, D. Gere, D. McManigill and H. Weaver, Papers presented at 1982 Pittsburgh Conference on Analytical Chemistry, Papers available as Hewlett-Packard Co. Publication No. 43-5953-1647 (1982).

78. M. Novotny and S. R. Springston J. Chromatogr.. 279417 (1983).

79. K. Grob, J. High Res. Chrom. \& Chrom. Commun. 6178 (1983).

80. J. C. Fjeldsted, R. C. Kong, and M. L. Lee, J. Chromatogr. 279449 (1983).

81. J. C. Fjeldsted, B. E. Richter, W. P. Jackson and M. L. Lee, J. Chromatogr. 279423 (1983). 
82. R. H. Shafer and P. R. Griffiths, Anal. Chem. 551939 (1983).

83. F. P. Schmitz, H. Hilgers and E. Klesper, J. Chromatogr. 267267 (1983).

84. L. R. Snyder, J. Chromatogr. 92223 (1974).

85. L. R. Snyder, J. Chrom. Sci, 16223 (1978).

86. A. Wilsch and G. M. Schneider, Fresenius Z. Anal. Chem. 316265 (1983).

87. A. Wilsch, R. Feist and G. M. Schneider, Fluid Phase Equilib. 10 299 (1983).

88. J. C. Fjeldsted and M. L. Lee, Anal. Chem. 56 619A (1984).

89. M. Novotny, M. L. Lee, P. A. Peaden, J. C. Fjeldsted, and S. R. Springston, U. S. Patent 4,479,380, Brigham Young University (1984).

90. R. C. Kong, C. L. Woolley, S. M. Fields, and M. L. Lee, Chromatographia 18362 (1984).

91. R. C. Kong, S. M. Fields, W. P. Jackson, and M. L. Lee, J. Chromatogr.. 289105 (1984).

92. S. M. Fields, R. C. Kong, J. C. Fjeldsted, M. L. Lee and P. A. Peaden, J. High Res. Chrom. \& Chrom. Commun. 7312 (1984).

93. S. M. Fields, R. C. Kong, M. L. Lee and P. A. Peaden, J. High Res. Chrom. \& Chrom. Commun 7423 (1984).

94. S. R. Springston and M. Novotny, Anal. Chem. 561762 (1984).

95. F. P. Schmitz, D. Leyendecker and E. Klesper, Berichte Bunsenges Phys. Chem 88912 (1984).

96. D. Leyendecker, F. P. Schmitz, and E. Klesper, J. Chromatogr. 315 19 (1984).

97. F. P. Schmitz, H. Hilgers, D. Leyendecker, B. Lorenschat, U. Setzer and E. Klesper, J. High Res. Chrom. Chrom. Commun. I 590 (1984).

98. G. M. Schneider, Ber. Besunges Phys. Chem. 88841 (1984).

99. T. A. Norris and M. G. Rawdon, Anal. Chem. 561767 (1984).

100. B. Wenclawiak and F. Bickmann, Fresenius Z. Anal. Chem. 319305 (1984). 
101. Y. Hirata, J. Chromatogr. 31539 (1984).

102. B. W. Wright, H. R. Udseth, R. D. Smith and R. N. Hazlett, J. Chromatogr. 314253 (1984).

103. B. W. Wright and R. D. Smith, Chromatographia. 18542 (1984).

104. M. G. Rawdon and T. A. Norris, American Lab. 1617 (1984).

105. T. L. Chester, J. Chromatogr..., 299424 (1984).

106. R. D. Smith, H. T. Kalinoski, H. R. Udseth, and B. W. Wright Anal. Chem. 562476 (1984).

107. R. D. Smith, H. R. Udseth and H. T. Kalinoski, Anal. Chem. $\underline{56} 2971$ (1984).

108. K. H. Shafer, S. L. Pentoney and P. R. Griffiths, J. High Res. Chrom. \& Chrom. Commun. 7707 (1984).

109. Y. Hirata and F. Nakata, J. Chromatogr. 295315 (1984).

110. T. Takeuchi, D. Ishll, M. Saito and M. Hibi J. Chromatogr. 295323 (1984).

111. Y. Hirata, J. Chromatogr. 31531 (1984).

112. Y. Hirata, J. Chromatogr. 31539 (1984).

113. B. E. Richter, J. High. Res. Chrom, and Chrom. Commun. 897 (1985).

114. B. W. Wright and R. D. Smith, J. High Res. Chrom. Chrom. Commun. $\underline{8}(1985)$.

115. R. D. Smith, H. R. Udseth and B. W. Wright, J. Chrom. Sci. 23192 (1985).

116. C. M. White and R. K. Houck, J. High Res. Chrom. \& Chrom. Commun. ㅇ 293 (1985).

117. J. C. Kuei, B. J. Tavbet, W. P. Jackson, J. S. Bradshaw, K. E. Markides and M. L. Lee, Chromatographia 2025 (1985).

118. C. Fujimoto, Y. Hirate and K. Jinno, J. Chromatogr. 33247 (1985).

119. C. C. Johnson, J. W. Jordan, L. T. Taylor, and D. W. Vidrine Chromatographia 20717 (1985).

120. E. J. Guthrie and H. E. Schwartz J. Chrom. Sci. 21236 (1986). 
121. S. L. Pentoney, Jr., R. H. Shafer, P. R. Griffiths and R. Fuoco, J. High Res. Chrom. \& Chrom. Commun. 9135 (1986).

122. S. L. Pentoney, K. H. Shafer and P. R. Griffiths, J. Chrom. Sci. 24 230 (1986).

123. W. R. West and M. L. Lee, J. High Res. Chrom. \& Chrom. Commun $\underline{9}$ 161 (1986).

124. R. L. Eatherton, M. A. Morissey, W. F. Siems and H. H. Hill, Jr., J. High Res. Chrom. Chrom. Commun. 9154 (1986).

125. E. D. Lee and J. D. Henion, J. High Res. Chrom. \& Chrom. Commun. 9 172 (1986).

126. D. W. Later, B. E. Richter, D. E. Knowles and M. R. Anderson, J. Chrom. Sci. 24249 (1986).

127. K. E. Markides, S. M. Fields and M. L. Lee, J. Chrom. Sci. 24254 (1986).

128. W. P. Jackson and D. W. Later, J. Bigh Res. Chrom. \& Chrom. Commun. 9175 (1986).

129. T. L. Chester and D. P. Innis, J. High Res. Chrom. \& Chrom. Commun. 9178 (1986).

130. S. J. Deluca, K. J. Voorhees, T. A. Langworthy and G. Holzer, J. High Res. Chrom. \& Chrom. Commun. 9182 (1986).

131. M. Proot, P. Sandra and E. Geeraert, J. High Res. Chrom. \& Chrom. Commun. 9189 (1986).

132. H. E. Schwartz, J. W. Higgins and R. G. Brownlee, LC-GC 4639 (1986).

133. R. E. Markides, R. S. Johnson, B. J. Tarbet, J. S. Bradshaw and M. L. Lee, Pittsburgh Conference 1986, Paper 746.

134. E. J. Guthrie, Pittsburgh Conference 1986, Paper 747.

135. M. L. Kumar, C. P. Wagner, R. M. Ravey, and R. K. Houck, Pittsburgh Conference 1986, Paper 748.

136. E. M. Calvey, C. W. Thorpe and A. E. Pohland, Pittsburgh Conference 1986, Paper 750.

137. J. W. Jordan, L. T. Taylor, M. Ashraf-Khorassani and J. W. Hellgeth, Pittsburgh Conference 1986, Paper 751. 
138. M. Ashraf-Khorassani, J. W. Hellgeth and L. T. Taylor, Pittsburgh Conference 1986, Paper 752 .

139. K. G. Owens and J. P. Reilly, Pittsburgh Conference 1986, Paper 753.

140. C. H. Sin, H. M. Pang and D. M. Lubman, Pittsburgh Conference 1986, Paper 754 .

141. P. Jusforgues and M. Perrut, Pittsburgh Conference 1986, Paper 755.

142. B. E. Richter, Pittsburgh Conference 1986, Paper 514.

143. T. L. Chester, Pittsburgh Conference 1986, Paper 515.

144. B. W. Wright, C. R. Yonker, H. T. Kalinoski and R. D. Smith, Pittsburgh Conference 1986, Paper 517.

145. Emory Leaseburg, Personal Communication.

146. R. D. Smith, ACS Summer Analytical Symposium, 1986, Salt Lake City, UT.

147. V. Pretorius and J. C. Davidtz, J. High Res. Chrom. \& Chrom. Comm. 2. 703 (1979).

148. S. R. Lipsky, W. S. McMurray, M. Hernandez, J. E. Purcell and K. A. Billeb, J. Chrom. Sci., 181 (1980).

149. A. V. Kisler, Russ. J. Phys. Chem. 38, 1501 (1964).

150. J. R. Hutchins, III and R. V. Harrington in "Encyclopedia of Chemical Technology", 2nd ed. vol. 10, Kirk-Othmeh, ed., John wiley \& Sons (1966) 533.

151. R. H. Dalton, J. Chem. Ed. 40, 99 (1963).

152. P. Raiser, Appl. Opt. 16701 (1977).

153. R. E. Jarger, Ceram. Bull. 55270 (1976).

154. F. S. Yang, J. High Res. Chrom. \& Chrom. Commun. 389 (1980).

155. W. Jennings, J. High Res. Chrom. \& Chrom. Commun. 3601 (1980).

156. V. Pretorius, J. C. Davitz and D. H. Desty, in "Proceedings of the Fourth International Symposium on Capillary Chromatography," R. E. Kaiser, ed., Huthig, Heidelberg (1981) 201. 
157. S. R. Lipsky, 1986 Pittsburgh Conference, New Product from Quadrex Co.

158. R. Dandenau, P. Bente, T. Rooney and R. Hiskes, Am. Lab, 1161 (1979).

159. L. T. Zhuravler, A. V. Kieseler, V. P. Naidina and A. L. Polyakov, Russ. J. Phys. Chem. 371216 (1963).

160. M. I. Hair and w. Hertl, J. Phys. Chem. 734269 (1969).

161. M. L. Hair, J. Non-Cryst. Solids, 19299 (1975).

162. M. L. Hair in "Infrared Spectroscopy in Surface Chemistry," Marcel Dekker, New York (1967) 79.

163. B. W. Wright, M. L. Lee and G. M. Booth, Chromatographia 15584 (1982).

164. M. R. Baslia, J. Chem. Phys, 351151 (1961).

165. J. B. Peri and A. L. Hensley, Jr., J. Phys. Chem. 722926 (1968).

166. J. B. Peri, J. Phys. Chem. 702937 (1966).

167. A. W. Adamson, "Physical Chemistry of Surfaces, 4th Ed." John Wiley and Sons, New York (1982) 338.

168. K. D. Bartle, B. W. Wright and M. C. Lee, Chromatographia 14, 387 (1981).

169. M. W. Ogden and H. M. McNair, Journal HIgh Res. Chrom. \& Chrom. Commun, 8326 (1985).

170. W. Jennings, J. High Res. Chrom. \& Chrom. Commun, 4601 (1981).

171. P. Sandra, M. Verstappe and M. Verzele, J. High Res. Chrom. \& Chrom. Commun., 1. 28 (1978).

172. J. D. Schieke, N. R. Comins and V. Pretorius, J. Chromatogr, 112, 97 (1975).

173. A. Vezele, G. Redant, M. van Roelenboch, M. Verstappe and P. Sandra, in "Proceedings of the Fourth International Symposium on Capillary Chromatography," R. E. Kaiser, ed. Huthig, Heidelberg (1981) 239.

174. K. Grob and G. Grob, J. Chromatogr., 125, 471 (1976).

175. K. Grob, Jr., G. Grob and R. Grob, J. High Res. Chrom, \& Chrom. Commun.. 1149 (1978). 
176. R. C. M. de Nijs, G. A. F. M. Rutten, J. J. Franken, R. P. M. Dooper, and J.A. Rijks, J. High. Res. Chrom.\& Chrom. Commun., 2 447 (1979).

177. A. I. German, C. D. Pfaffenberger, J. P. Thenot, M. G. Horning, and E. C. Horning, Anal. Chem., 45, 930 (1973).

178. R. D. Dandenan and E. H. Zerenner, J. High Res. Chrom.\& Chrom. Commun., 2, 351 (1979).

179. T. I. Chester, D. P. Innis and G. D. Owens, Anal. Chem,, 57, 2243-7 (1985).

180. R. K. Iler, The Chemistry of Silica-Solubility, Polymerization, Colloid and Surface Properties, and Biochemistry, John wiley and Sons, New York 1979.

181. W. A. Aue, C. R. Hastings and S. Rapila, J. Chromatogr., 77299 (1973).

182. D. A. Cronin, J. Chromatogr., 97263 (1974).

183. G. Schomburg, H. Husmann and F. Weeke, J. Chromatogr, 9963 (1974).

184. L. Blomberg, J. Chromatogr., 115, 365 (1975).

185. G. Schombug, H. Husmann and F. Weeke, Chromatographia, 10580 (1977).

186. R. F. Arrendale, G. W. Chapman and O. T. Chortyk, J. Agric. Food Chem., 31, 1334 (1983).

187. R. F. Arendale, R. F. Severson and O. T. Chortyk, J. High Res. Chrom. Chrom. Commun., 6436 (1983).

188. M. Verzele, J. High Res. Chrom. \& Chrom. Commun, 2 647 (1979).

189. K. Grob, G. Grob, and K. Grob, Jr., J. High Res. Chrom. \& Chrom. Commun., 231 (1979).

190.. M. Novotny, L. Blomberg, and K. D. Bartle, J. Chrom. Sci., 890 (1970).

191. G. Schomburg, H. Husmann and H. Borwitzky, Chromatographia, 12, 651 (1979). 
192. M. Verzele, G. Redant, M. van Roelenbosch, M. Godefroot, M. Verstappe and $P$. Sandra, in "Fourth International Symposium on Capillary Chromatography," R. E. Kaiser, ed. Huethig, Heidelberg, 1981, p. 239.

193. K. A. Andrianov, "Metalorganic Polymers," Interscience Publishers, New York (1965).

194. T. J. Stark, R. D. Dandenam and L. Mering, Pittsburgh Conference 1980, Paper 2.

195. L. Blomberg, K. Markides and T. Wannman, J. High Res. Chrom. \& Chrom. Commun., 3527 (1980).

196. D. J. Shaw, "Introduction to Colloid and Surface Chemistry," 3rd ed., Butterworth, London (1980).

197. K. D. Bartle, B. W. Wright and M. L. Lee, Chromatographia, 14387 (1981).

198. CRC Handbook of Physics and Chemistry, 60th ed., CRC Press, Boca Raton (1980).

199. T. Boogaerts, M. Verstappe and M. Verzele, J. Chrom. Sci,, 10217 (1972).

200. J. Bouche and M. Verzele, Chromatographia, 10487 (1977).

201. M. J. E. Golay, in "Gas Chromatography 1958", D. H. Desty, ed., Academic Press, New York (1958) 36.

202. K. Grob, Making and Manipulating Capillary Columns for Gas Chromatography, Huethig, New York (1986).

203. G. A. F. M. Rutten and J. A. Rijks, J. High Res. Chrom. \& Chrom. Commun., 1279 (1978).

204. P. Sandra and M. Vezele, Chromatographia, 11102 (1978).

205. C. H. Lochmuller and J. D. Fisk, J. High Res. Chrom. Chrom. Commun., 4232 (1981).

206. R. R. Kim, I. Ghadui and A. Zlatkis, J. High Res. Chrom, Chrom, Commun., $5571(1982)$.

207. K. Grob and G. Grob, J. High Res. Chrom. Chrom. Commun., 5119 (1982). 
208. I. Blomberg, J. Buijten, G. Gawdick and T. Wännman, Chromatographia, 11521 (1978).

209. G. Schomburg, H. Husmann, S. Ruthe and M. Herraiz, Chromatographia, 15599 (1982).

210. B. W. Wright, P. A. Peaden, M. L. Lee and T. Stark, J. Chromatogr, 248, 17 (1982).

211. B. E. Richter, J. C. Kuei, N. J. Park, S. J. Crowley, J. S. Bradshaw and M. L. Lee, J. High Res. Chrom. E Chrom. Commun,, 6 371 (1983).

212. K. Markides, L. Blomberg, J. Buijten and T. Wănnman, J. Chromatogr..., 53254 (1983).

213. J. Buijten, L. Blomberg, K. Markides and T. Wännman, Chromatographia, 16183 (1982).

214. K. Grob, Jr., G. Grob and K. Grob, J. Chromatogr,, 156 I (1978).

215. K. Grob, G. Grob and K. Grob, Jr., J. Chromatogr., 21913 (1981).

216. M. W. Ogden, Ph.D. Dissertation, Virginia Polytechnic Institute and State University (1985).

217. G. Rutten, A. van der Ven, J. de Haan, and J. Rijks, J. High Res. Chrom. \& Chrom. Commun., I 607 (1984).

218. K. Grob, Jr. and H. P. Neukom, J. Chromatogr, 323237 (1985).

219. K. Grob, Jr., H. P. Neukom and M. L. Riekkola, J.High Res. Chrom. \& Chrom. Commun., I 319 (1984).

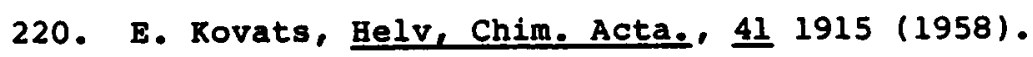

221. W. O. McReynolds, J. Chrom. Sci, 8, 685 (1970). 


\section{The vita has been removed from the scanned document}

